\title{
L-Arginine treatment during acute and long-term endotoxemia in the pig : effects on jejunal motility and interorgan protein, arginine and nitric oxide metabolism
}

Citation for published version (APA):

Bruins, M. (2001). L-Arginine treatment during acute and long-term endotoxemia in the pig : effects on jejunal motility and interorgan protein, arginine and nitric oxide metabolism. [Doctoral Thesis, Maastricht University]. Universiteit Maastricht. https://doi.org/10.26481/dis.20011220mb

Document status and date:

Published: 01/01/2001

DOI:

$10.26481 /$ dis.20011220mb

Document Version:

Publisher's PDF, also known as Version of record

Please check the document version of this publication:

- A submitted manuscript is the version of the article upon submission and before peer-review. There can be important differences between the submitted version and the official published version of record. People interested in the research are advised to contact the author for the final version of the publication, or visit the DOI to the publisher's website.

- The final author version and the galley proof are versions of the publication after peer review.

- The final published version features the final layout of the paper including the volume, issue and page numbers.

Link to publication

\footnotetext{
General rights rights.

- You may freely distribute the URL identifying the publication in the public portal. please follow below link for the End User Agreement:

www.umlib.nl/taverne-license

Take down policy

If you believe that this document breaches copyright please contact us at:

repository@maastrichtuniversity.nl

providing details and we will investigate your claim.
}

Copyright and moral rights for the publications made accessible in the public portal are retained by the authors and/or other copyright owners and it is a condition of accessing publications that users recognise and abide by the legal requirements associated with these

- Users may download and print one copy of any publication from the public portal for the purpose of private study or research.

- You may not further distribute the material or use it for any profit-making activity or commercial gain

If the publication is distributed under the terms of Article 25fa of the Dutch Copyright Act, indicated by the "Taverne" license above, 


\section{L-Arginine Treatment during Acute and Long- Term Endotoxemia in the Pig}

Effects on Jejunal Motility and Interorgan Protein, Arginine and Nitric Oxide Metabolism 
Cover: $\quad$ Appearance of arginine crystallites under a polarized light microscope

Printing: $\quad$ Ponsen \& Looijen, Wageningen, The Netherlands
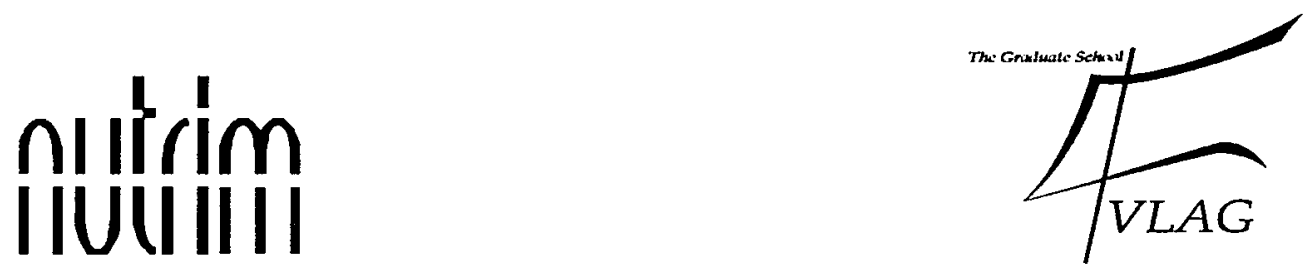

The study presented in this thesis was performed at the Nutrition and Toxicology Research institute Maastricht (NUTRIM), which participates in the Graduate School VLAG (Food Technology, Agrobiotechnology, Nutrition and Health Sciences), accredited by the Royal Netherlands Acedemy of Arts and Sciences 


\title{
L-Arginine Treatment during Acute and Long- Term Endotoxemia in the Pig
}

Effects on Jejunal Motility and Interorgan Protein, Arginine and Nitric Oxide Metabolism

\author{
PROEFSCHRIFT \\ ter verkrijging van de graad van doctor \\ aan de Universiteit Maastricht, \\ op gezag van de Rector Magnificus, \\ Prof. Dr. A.C. Nieuwenhuijzen Kruseman \\ volgens het besluit van het College van Decanen \\ in het openbaar te verdedigen \\ op donderdag 20 december 2001 om 10.00 uur
}

door

Maaike Bruins

Geboren 20 april 1970 te Chêne-Bougeries 


\section{Promotor:}

Prof. Dr. P.B. Soeters

Prof. Dr. W.H. Lamers (Universiteit van Amsterdam)

\section{Co-promotor:}

Dr. N.E.P. Deutz

\section{Beoordelingscommissie:}

Prof. Dr. P. de Leeuw (voorzitter)

Prof. Dr. L.M. Akkermans (Universiteit Utrecht)

Prof. Dr. L. Cynober (Université Paris)

Dr. A.J. Meijer (Universiteit van Amsterdam)

Prof. Dr. G. Ramsay

Prof. Dr. R.W. Stockbrügger

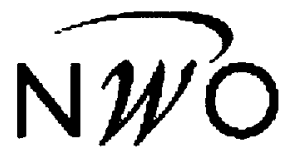

The studies described in this thesis were supported by a research grant (902-23-098) from the Netherlands Organization for Scientific Research. 
The Road goes ever on and on, Down from the door where it began. Now far ahead the Road has gone, And I must follow, if I can, Pursuing it with weary feet, Where many paths and errands meet. And whither then? I cannot say.

J.R.R. Tolkien (The Lord of the Rings)

Aan allen die mij bijstonden 


\section{CONTENTS}

CHAPTER 1 General introduction arginine and nitric oxide in sepsis

CHAPTER 2 General introduction sepsis and measurements models

CHAPTER 3 Alterations in organ protein turnover and substrate fluxes during endotoxemia

CHAPTER 4 Endotoxemia affects organ protein metabolism differently during prolonged feeding in pigs

CHAPTER 5 L-Arginine supplementation in the pig decreases liver protein turnover and increases hindquarter protein turnover both during and after endotoxemia

CHAPTER 6 In vivo measurements of organ nitric oxide production during hyperdynamic endotoxemia in pigs determined from stable isotope conversion

CHAPTER 7 L-Arginine supplementation increases organ nitric oxide production in a pig model of hypermetabolic endotoxemia

CHAPTER 8 Effects of arginine supplementation on organ urea cycle intermediate metabolism in endotoxemic pigs

CHAPTER 9 The effect of prolonged hyperdynamic endotoxemia on jejunal motility in fasted and enterally fed pigs

CHAPTER 10 Effects of long-term intravenous and intragastric L-arginine intervention on jejunal motility and visceral nitric oxide production in the endotoxemic pig

CHAPTER 11 General discussion

List of abbreviations

List of enzyme codes

Dankwoord

List of Publications

Curriculum vitae 


\title{
ARGININE AND NITRIC OXIDE IN SEPSIS
}

\author{
Maaike J Bruins \\ Peter B Soeters \\ Nicolaas EP Deutz \\ Wouter J De Jonge *
}

Department of Surgery, Maastricht University, The Netherlands

-Department of Anatomy \& Embryology, University of Amsterdam, AMC, The Netherlands 


\section{Arginine}

Arginine is considered an indispensable amino acid in carnivores (194) and a conditionally indispensable amino acid in omnivores (193). A conditionally indispensable amino acid is defined as an amino acid that becomes indispensable when de novo capacities of synthesis are insufficient to cover increased needs occurring for example during growth (92) or in response to trauma and sepsis (192).

\subsection{Cellular arginine transport}

Occurrence and kinetics arginine transporters Transport of arginine, ornithine, and lysine across plasma membranes is largely mediated through the sodium independent amino acid transport system formerly designated $\mathrm{y}^{+}$and $\mathrm{y}^{+} \mathrm{L}$. Four homologous human and rodent genes make up the family of cationic amino acid transporters (CATs) which encode system $\mathrm{y}^{+}$: CAT-1, CAT $2 \mathrm{~A}$ and a splice variant CAT-2B, CAT-3 (59) and recently discovered CAT-4 (9). The CAT-1 transporter is widely expressed in mammalian tissues but is virtually absent in hepatocytes (119). In hepatocytes in the normal physiological state, CAT- 1 activity is barely detectable whereas the low affinity isoform of the CAT-2 transporter, CAT-2A, is abundantly expressed (119). The kinetic constants $V_{\text {max }}$ (maximal transport rate when excess substrate is present) and $K_{m}$ (substrate concentration at which the transport rate is half of the maximum) for arginine uptake by a cell depend on the CAT-transporter isoform distribution characteristics for that cell type. The CAT-2A liver isoform mediates cationic amino acid transport at 10-70 fold higher apparent $K_{m}$ and $V_{\max }$ than the CAT-1 and $2 B$ isoforms (202). The $K_{m}$ value of hepatocytes is well above the normal plasma concentration of arginine. This low affinity however, does not make the transport of arginine into these cells the rate-limiting step for arginine metabolism since CAT-2A has a high $V_{\max }$. This provides a kinetic barrier separating the extrahepatic arginine pool from the active cytoplasmic enzymes of the hepatic urea cycle.

Regulation arginine transporters Transporter CAT-2 can be induced by inflammatory cytokines in vascular smooth muscle cells (78), hepatocytes (148) and macrophages (102). Lipopolysaccharide (LPS) also has been shown to stimulate arginine transport in endothelial cells and vascular smooth muscle cells of the lung, heart and kidney (85) and in macrophages (169). LPS treatment in rats induced a two-fold stimulation of the arginine transport in hepatocytes $(97)$ by increasing the $V_{\max }$ without modifications of transport affinity, implicating an upregulation of the amount of arginine transporters (97).

The affinity for the D-arginine is 20 times less than for the L-arginine (202). On the contrary, L-homoarginine is specifically transported with high affinity, making it to a useful 
probe to study CAT-1 activity, as physiological concentrations of L-homoarginine are low (52). Cationic amino acids other than arginine such as lysine, ornithine, leucine, are effective competitive inhibitors of arginine uptake by CAT. There is a steric constraint for arginine binding to the CAT-1 transporter as $\alpha$-methylation of arginine completely abrogates arginine binding (106). Nitric oxide synthase (NOS) inhibitors, including the arginine analogues omega-nitro-L-arginine methyl ester (L-NAME) and N-methyl-Larginine (L-NMA), can competitively inhibit arginine uptake by the CAT transporters, and thereby limit the availability of arginine in the cell (97).

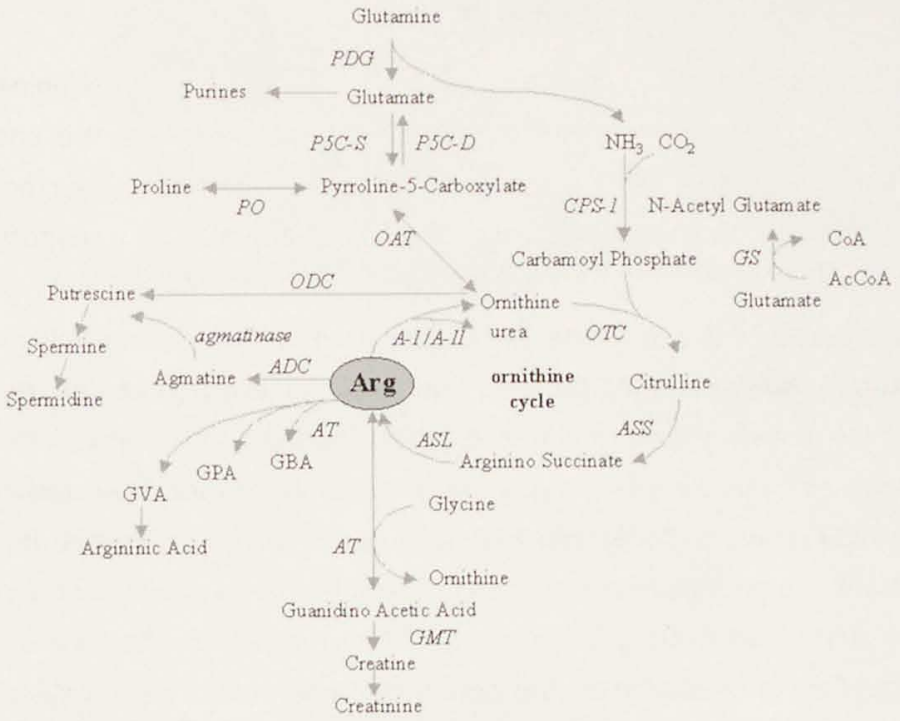

Figure 1. Drawing of the ornithine cycle schematically showing the involvement of different compounds. The different enzymes are expressed in a different extent in the diverse organs.

\subsection{Arginine synthesis}

In mammals, arginine is an intermediate of the ornithine-cycle (Figure 1). Citrulline is converted into arginine via arginino succinate by the enzymes argininosuccinate synthase (ASS) and agininosuccinate lyase (ASL). In the adult, ASS and ASL are predominantly found in the liver, but also in the kidney. The liver has high arginase expression responsible for the rapid conversion of arginine into ornithine and urea (126) (see also: 1.6 Organ characteristics of arginine metabolism; Liver). Because the high arginase content prevents the release of arginine from the liver into the circulation, net arginine output from the liver is absent. As shown in Figure 2 the kidney plays a major role in the de novo synthesis of arginine from citrulline, which is taken up from the circulation $(60,203)$. 


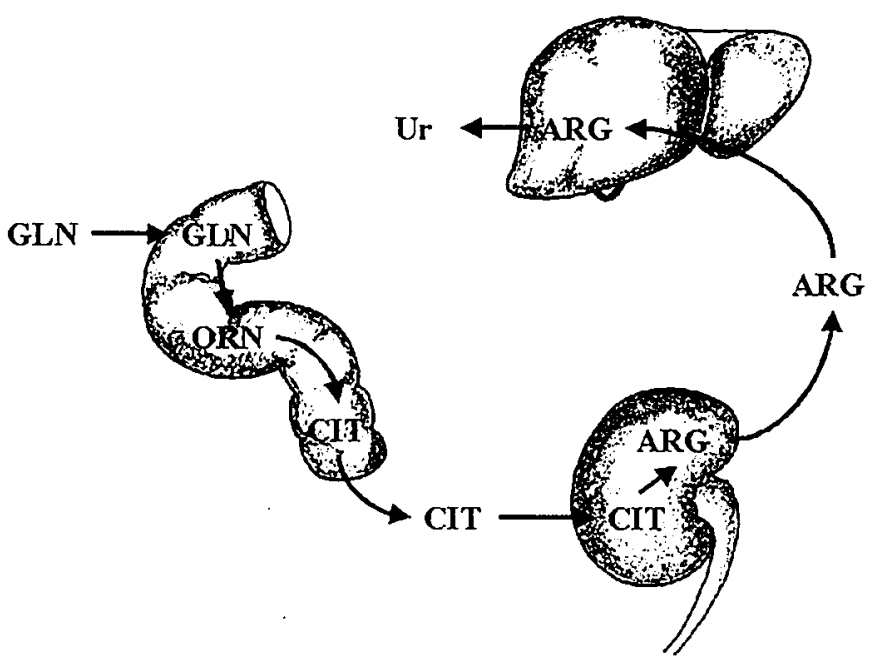

Figure 2. Involvement of the gut, kidney and liver in the arginine de novo synthesis.

The main source of this arginine is citrulline that is synthesized in the gut via ornithine from glutamine/glutamate or proline. Especially in the jejunum, three enzymes act in concert in the net synthesis of citrulline (132). Proline and glutamine/glutamate in the gut are the source of pyrroline-5-carboxylate which is a common intermediate in the ornithine synthesis (208) (Figure 1). Pyrroline-5-carboxylate can be converted into ornithine by the enzyme ornithine aminotransferase (OAT), which also exclusively occurs in the gut (197). Ammonia is derived from glutamine and forms, together with bicarbonate, carbamoylphosphate. This is catalyzed by the action of carbamoyl-phosphate synthetase (CPS-1), which requires $\mathrm{N}$-acetyl glutamate for activity. The carbamoyl group is transferred to ornithine to form citrulline in a reaction catalyzed by ornithine transcarbamylase (OTC). Citrulline, formed by the intestine, is released into the portal circulation. Citrulline is not extracted by the liver (186) and released into the systemic circulation from which it is taken up by the kidney where citrulline is used for the synthesis of arginine.

\subsection{Arginine catabolism}

Four degrading pathways of arginine distinguished (Figure 1). The first, and main, is mediated by arginase essential for the synthesis of urea and, via ornithine, of polyamines and proline. The second pathway is mediated by arginine:glycine amidinotransferase (AT) that catalyzes the first reaction in the biosynthesis of creatine/creatinine or catalyzes guanidino compound formation. A third degrading pathway by arginine decarboxylase $(A D C)$ results in the synthesis of agmatine. The final pathway, via nitric oxide synthase (NOS), yields citrulline and nitric oxide (NO) in equal amounts. 

biosynthesis of ornithine and guanidino acetic acid (GAA), which is the precursor of creatine (163). This conversion principally occurs in the kidney and pancreas $(125,163)$ by the enzyme arginine:glycine amidinotransferase (AT). GAA can subsequently be released and transported to the liver. In the liver, this GAA can become methylated by $S$ adenosylmethionine:guanidinoacetate methyltransferase (GMT) to form creatine. Creatine is transferred to the muscle and nerves where it serves, bound to phosphate (creatine-phosphate), an important role in energy metabolism. When it is further dehydrated, creatinine is produced and excreted in urine. About $13.3 \mathrm{mmol}$ arginine per day, $\sim 10 \%$ of the whole body arginine flux, is needed for the synthesis and maintenance of creatine (33), therefore, constituting a major arginine degrading pathway.

Agmatine

Agmatine is another metabolite of arginine, formed via the enzyme ADC. Agmatine and ADC activity have been reported to be present in brain, liver, kidney, intestine, adrenal glands and macrophages $(170,183)$, but the function of agmatine is not well understood. Agmatine can be hydrolyzed by agmatine urohydrolase (agmatinase) to putrescine, and hence agmatine is a metabolic precursor for the biosynthesis of higher polyamines (82). Agmatine may suppress cell proliferation by inhibiting ODC activity and subsequent polyamine biosynthesis (171). In addition, agmatine may play a role in cell signaling by controlling NO production by inhibiting NO synthases (74).

Guanidino compounds Arginine also serves as amidine donor in the formation of guanidino compounds. The enzyme AT accepts a wide variety of substrates as amidine acceptors. In addition to the physiological substrate glycine, it accepts the glycine analogues $\alpha$-aminoproprionic acid, $\gamma$-aminobutyric acid or $\delta$-aminovaleric acid can function as substrate, yielding guanidinopropionic acid (GPA), guanidinobutyric acid (GBA), and guanidinovaleric acid (GVA), respectively $(121,212)$. Most probably, GVA can be subsequently hydrogenated to form argininic acid (121). In hyperargininemic patients, increased levels of guanidino compounds were observed (121).

Urea Arginine is hydrolyzed to urea and ornithine in equal amounts by the action of arginase, of which two main subtypes are known. Hepatic arginase I (Al) is primarily found in liver, but also in extrahepatic tissue i.e. in erythrocytes (188) and macrophages (166). Extra-hepatic arginase II (AII) is found in virtually all organs except in the liver (100). Urea is for most ureotelic mammals a way to excrete nitrogen compounds resulting from the detoxification of ammonia formed in the breakdown of amino acids. Arginine possibly also regulates urea synthesis by allosteric activation of the synthesis of $\mathrm{N}$-acetyl glutamate, which, in turn, activates the enzyme CPS-1 (126). Ornithine, may be used for further conversion in urea cycle, but also for other purposes such as proline and polyamine synthesis (183). The $K_{m}$ value for arginase is above $1 \mathrm{mM}(94)$. 
Proline Ornithine can be converted by the enzyme ornithine aminotransferase (OAT) and the subsequently formed pyrroline-5-carboxylate via pyrroline-5-carboxylate reductase to proline. Proline is oxidized back into pyrroline-5-carboxylate by proline oxidase. These reactions occur in the mitochondrion of the enterocyte (211). Proline is a secondary amino acid, and is a precursor in collagen synthesis (156), which implies that arginine has also a role in wound healing and cell proliferation (12).

Polyamines

Ornithine also serves as precursor for polyamine synthesis and is decarboxylated by the rate-limiting enzyme ornithine decarboxylase (ODC) yielding putrescine, the first polyamine in the biosynthetic pathway that can further be converted to spermine and spermidine. Polyamine synthesis has been demonstrated to correlate with increased arginase and ODC activity in the kidney (120) and the small intestine (210). The rapid inducibility of ODC by growth stimuli and its short half-life (183) allow the cell to rapidly respond to growth stimuli. Polyamines are known to exhibit regulatory properties in cell multiplication and proliferation $(154,171)$ and, by that means, are involved in tissue growth and wound repair.

Nitric oxide Another, yet minor, pathway in the arginine degradation is the NO synthase (NOS) pathway in which the free-radical NO and citrulline are stoichiometrically formed by the action of NOS (131). NO is formed in parallel with citrulline by the oxidation of one of the two equivalent terminal guanidino nitrogen groups from arginine (104). In the first step, L-arginine is oxidized into L-hydroxy- $\mathrm{N}$-arginine and, in the second step, this intermediate product is rapidly converted into citrulline and NO. This conversion uses one NADPH and oxygen molecule and one NADP molecule is formed (Figure 3). Three distinct isoforms of NOS are recognized (Figure 3), representing three different gene products (143). Two of these isoenzymes, defined as cNOS, are expressed constitutively and are calcium-dependent, whereas one isoform, termed inducible NOS (iNOS or NOSII), is only synthesized de novo after stimulation and is not calcium-dependent. One cNOS subtype, named endothelial NOS (eNOS or NOS-III), is mostly membrane bound (133) and is present in several cells, mostly endothelial cells $(104,167)$. The other cNOS subtype is cytosolic and mostly present in neuronal tissue $(104,167)$ and, therefore, defined as neuronal NOS (nNOS or NOS-1). The NOS-II isoform is cytosolic and is expressed in a great variety of cells including macrophages $(82,133,167)$. The cNOS enzyme is permanently present in cells, synthesizing small amounts of NO. The NOS-II isoform is not present at basal levels but can be induced in response to inflammatory cytokines and endotoxin (167). The $\mathrm{K}_{\mathrm{m}}$ values reported for $\mathrm{cNOS}$ for NO biosynthesis from extracellular arginine approximated $1.6 \mu \mathrm{M}(130), 4.3 \mu \mathrm{M}(207)$, values of which are in about the same range for the NOS-II enzyme; $11 \mu \mathrm{M}(13), 22 \mu \mathrm{M}(77)$ and $49 \mu \mathrm{M}(94)$. 


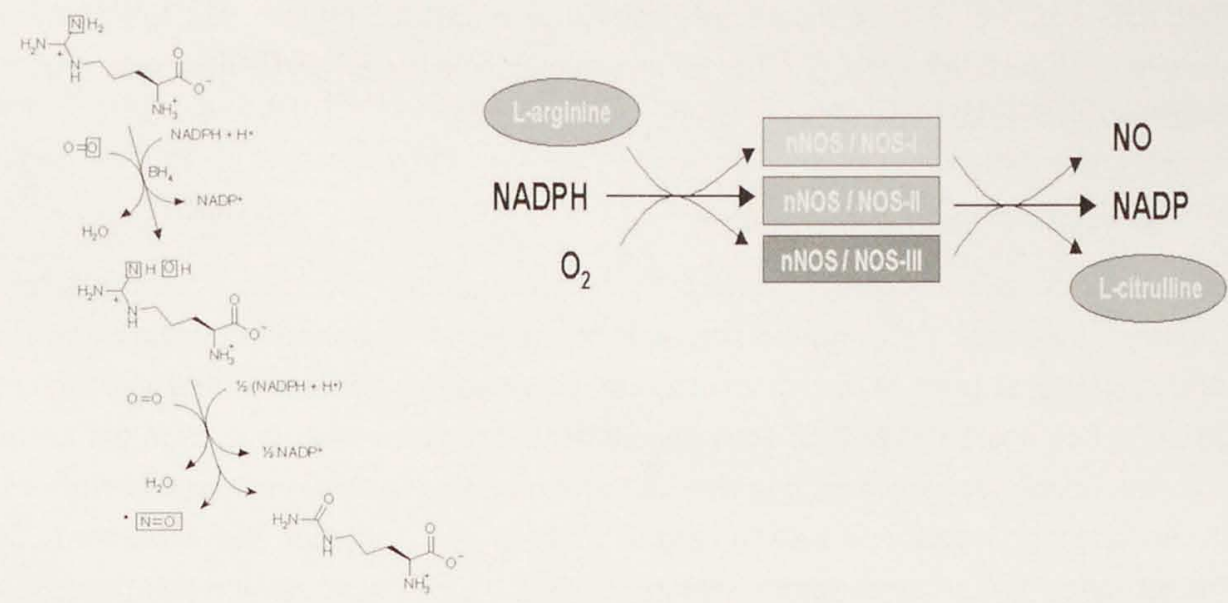

Figure 3. Schematic illustration of nitric oxide formation from L-arginine in the nitric oxide synthase pathway.

\section{Methods of NO and NOS measurements}

$\mathrm{NO}^{\circ}$ is a free oxygen radical and easily reacts with numerous substances and has, therefore, a half-life of only $0.1-10 \mathrm{sec}$. Because NO* rapidly reacts with other molecules, it is difficult to directly measure $\mathrm{NO}^{\circ}$. Therefore, end products of $\mathrm{NO}^{\circ}$ are used as an indicator of $\mathrm{NO}^{\circ}$ production. In whole blood, $\mathrm{NO}^{*}$ is rapidly metabolized by interaction with the heme group of hemoglobin to the stable end products of $\mathrm{NO}^{\circ}$, nitrite $\left(\mathrm{NO}_{2}{ }^{-}\right)$and nitrate $\left(\mathrm{NO}_{3}{ }^{-}\right)$(177). Since these metabolites can be excreted in the urine, their urinary output is often used as a measure of the endogenous production of $\mathrm{NO}^{\circ}$ although it has to be taken into account that these products can also be derived from dietary intake (81). When NO ${ }^{\circ}$ reacts with a thiol group of an amino acid in a protein, relatively stable nitrosothiols are formed (76) which can be identified by means of fluorescence techniques. Under certain conditions, $\mathrm{NO}^{*}$ rapidly reacts with superoxide $\left(\mathrm{O}_{2}{ }^{\circ}\right)$ to form the strong nitrating agent peroxynitrite $\left(\mathrm{ONOO}^{\circ}\right)$ (17). Peroxynitrite can decompose to hydroxyl radical $\left(\mathrm{OH}^{\circ}\right)$ and nitrogen dioxide $\left(\mathrm{NO}_{2}{ }^{\circ}\right)$ giving rise to nitrate $\left(\mathrm{NO}_{3}{ }^{-}\right)$and hydrogen $\left(\mathrm{H}^{+}\right)$.

$$
\begin{aligned}
& \mathrm{O}_{2}{ }^{-}+\mathrm{NO}^{\circ} \rightarrow \mathrm{ONOO}^{\circ} \\
& \mathrm{ONOO}^{*} \rightarrow \mathrm{OH}^{\cdot}+\mathrm{NO}_{2}{ }^{-} \\
& \mathrm{NO}_{2}{ }^{-}+\mathrm{H}_{2} \mathrm{O} \rightarrow \mathrm{NO}_{3}{ }^{-}+2 \mathrm{H}^{+}
\end{aligned}
$$

Peroxynitrite has a strong reactivity with free or protein-bound tyrosine residues in proteins. The occurrence of nitrotyrosine, which is a product of tyrosine nitration by peroxynitrite has been considered as evidence for in vivo formation of peroxynitrite and, thus, of NO (145). Expression of the NO synthesizing enzyme NOS can be detected immunohistochemically (18) using an antibody raised against a synthetic peptide of NOS. 
Tissue expression of mRNA encoding NOS can be measured by Northern blot analysis and reverse transcription PCR (36). In situ hybridization was performed with probes corresponding to cDNA sequences common to NOS (41).

\subsection{Endocrine activity of arginine}

Arginine exerts a secretagogue effect by stimulating the secretion of the pituitary hormones, the pancreatic hormones and the adrenal hormones. Pituitary growth hormone $(\mathrm{GH})$ has been shown to increase in response to intravenous administration of arginine in humans $(109,127)$. Dietary supplemented arginine that appears in the portal vein is extensively degraded by the liver (33) and normally will not result in a rise of $\mathrm{GH}$. Arginine-induced $\mathrm{GH}$ release were proposed to be dose-dependent (39). Observed $\mathrm{GH}$ activation after oral arginine administration $(21,98)$, therefore, possibly result from high portal arginine levels escaping liver arginase. Arginine was also found to exert a pituitary hormone effect by the endogenous release of prolactin, both via intravenous (160) and via oral (21) arginine administration. Furthermore, arginine administration has been reported to stimulate the secretion of the pancreatic hormones insulin $(62,69)$, glucagon (149) pancreatic polypeptide (199), and the GH-inhibiting hormone somatostatine (187). Besides these pancreatic hormonal responses, arginine was also found to stimulate the secretion of catecholamines $(96,161)$ by the adrenal gland. Several studies have shown that the effects of arginine including the induction of vasodilatation, inhibition of platelet aggregation and blood viscosity are mediated, in part, by arginine induced endogenously released insulin $(79,108)$. Nevertheless, other positively charged amino acids stimulate insulin, prolactin and glucagon release as well (118). NO exerts a major influence on islet hormone secretion (2); NO was found a negative modulator of insulin release but a positive modulator of glucagon release.

\subsection{Involvement of arginine and its metabolites in metabolism}

Arginine may exert a regulatory function in metabolic processes as such, but also through its metabolites or via its effect on hormonal release and, thereby, may be involved in protein, carbohydrate and fat metabolism. The effects on protein and carbohydrate metabolism will be summarized.

Protein A high protein turnover has been put forward to facilitate a rapid response to adapt to changing conditions by means of rapid alterations of enzyme concentrations and elimination of damaged proteins (141). During disease and injury the organism responds by changing the rates in the synthesis and/or degradation, i.e. turnover of proteins. Translational initiation factors and the rate of translation of specific mRNAs and mRNA concentration are one of the many factors determining the rate of protein 
synthesis. Post-translational modification of proteins such as arginylation, glycosylation and phosphorylation affect protein function by protein breakdown. Protein synthesis and degradation however, are only the two extremes of a complex phenomenon that includes a variety of other protein modifications.

Supplementation of intravenous or enteral arginine is associated with alterations in protein metabolism. In traumatized rats, dietary arginine supplementation improved nitrogen balance and collagen synthesis (38). Arginine supplementation proved to stimulate muscle protein synthesis in tumor-bearing rats (146). These effects may, at least in part, be mediated by the arginine-mediated hormone release such as insulin and $\mathrm{GH}$, which are known to produce anabolic effects by inhibiting the loss of body protein and stimulating amino acid transport into the cells (124). Dietary supplementation with ornithine and ornithine $\alpha$-ketoglutarate (OKG) also was associated with improved nitrogen balance (56). Ornithine shares most of the immunostimulatory and secretagogue effects of arginine $(65,114)$. It is, therefore, likely that these compounds share the same cellular mechanism of action or that arginine acts via increasing the concentration of available ornithine. OKG has been successfully used as an enteral supplement in the treatment of catabolic states, including burn injury (50). OKG was extensively metabolized in these patients with the production of glutamine, arginine, and proline (116). OKG increased insulinemia and glucagonemia in healthy subjects (51). Ornithine, nevertheless, did not replace the effect of arginine on growth in dogs (49)). With respect to protein degradation, arginine may be involved in modulation of protein breakdown by post-translational arginylation followed by ubiquitin-proteasome pathway-mediated protein breakdown (24).

Arginine may also be involved in the regulation of protein metabolism through its metabolites NO, ornithine, polyamines, proline. I.e., ornithine serves as precursor in the synthesis of polyamines and proline. Polyamines are required for DNA synthesis and cell growth (93) and proline for reparative collagen synthesis (5). In rats of which the small bowel was resected, arginine supplementation increased the intestinal concentration of the polyamine putrescine but despite this, decreased protein synthesis rate (200). In vitro studies demonstrated that $\mathrm{NO}$ and the NO-generating compound S-nitroso-Nacetylpenicillamine inhibit hepatocyte total protein synthesis (48) probably via NOmediated depletion of endoplasmic reticulum calcium stores (61). Increasing evidence shows that NO metabolism is involved in oxidative damage of protein. NO appears to stimulate intracellular protein breakdown by post-translational modifications of plasma and tissue proteins (66). Furthermore, the arginine products NO and agmatine were demonstrated to inhibit cell proliferation by limiting polyamine synthesis via inhibition of the enzyme ODC and by inhibiting the expression of the putrescine transporter $(170,171$, 
190). The decreasing effects of arginine on polyamine-mediated cell proliferation may therefore be attributed to feedback inhibition by products of arginine breakdown.

Carbohydrate Many of the effects of arginine on glucose metabolism are reported to be mediated by NO. In the liver, NO synthesis by the hepatocyte was shown to inhibit liver gluconeogenesis $(179,180)$. In skeletal muscle, cytokines modulate the glucose transport by upregulation of the NO-mediated glucose transport (19). Arginine-induced release of pancreas hormones like insulin $(62,69)$, glucagon (149) and pancreatic polypeptide (199) may underlie many of the effects of arginine on carbohydrate metabolism. Increased blood glucose levels associated with arginine supplementation may results from an increase in the secretion of glucagon and subsequent increased glycogenolysis and gluconeogenesis (149) or may be due to $\mathrm{GH}$ secretion that diminishes glucose cell uptake. However, arginine-induced secretion of insulin increases glucose uptake by liver cells resulting in decreased plasma glucose levels.

\subsection{Organ characteristics of arginine metabolism}

Immune cells Among the class of immune cells, the monocytes (macrophages) are the most extensively described in relation to arginine and NO metabolism. Macrophages contain high levels of arginase and activated macrophages consume arginine to a large extent. NO production by inducible nitric oxide synthase (NOS-II) is increased in response to LPS and cytokines. Additionally, the rate of arginine biosynthesis from glutamine via citrulline may be increased by LPS stimulation of the macrophage (134). It appears that both the degree of activation and the concentration of extracellular arginine determine whether these cells produce high or moderate quantities of NO.

Liver In mammals, the major catabolic pathway to remove toxic ammonia is the production of non-toxic urea that is formed in the liver and excreted in urine. Besides the kidney, the liver is capable of de novo synthesizing arginine. However, arginine that enters the liver does not escape catabolism because of high hepatic arginase activity. Arginine as a product of the urea cycle is able to further accelerate the urea cycle by allosteric activation. Arginine activates the enzyme $\mathrm{N}$-acetylglutamate synthase (GC) which catalyzes $\mathrm{N}$-acetylglutamate production from glutamate (see also: 1.2 Arginine synthesis) which converts ornithine to citrulline.

Gastrointestinal tract

At birth the small intestine is the major site of arginine synthesis (22). In most mammalia neonates, the complete pathway to synthesize arginine exists in the small intestine and enterocytes of the intestinal tract are capable of producing arginine from citrulline. The citrulline that is released by the small intestine is 
synthesized from glutamine and ornithine (70). After weaning, the small intestine gradually becomes the major site of net citrulline production as ASS and ASL expression disappear (57). Of all mammals studied, the pig is the only species whose intestine still expresses ASS and ASL at an adult age and consequently, releases endogenously synthesized arginine into the circulation $(182,211)$. After weaning, this arrangement is compensated for by the gradually increasing capacity of the kidney to synthesize arginine from citrulline $(60)$.

Kidney In adult dogs (215), similar as in young pigs (own observations), in the post-absorptive state approximately $80 \%$ of the net arginine synthesis occurs in the kidney. The kidney extracts citrulline from the blood to convert it into arginine (Figure 2) in a stoichiometrical fashion (215). Aspartate, glutamine or glutamate serve as $\mathrm{N}$ donor for the guanidino group of arginine (60). The arginine synthesizing enzymes argininosuccinate synthase (ASS) and argininosuccinate lyase (ASL) are highly expressed in the kidney $(60)$. A correlation was found between the net uptake of citrulline and the net output of arginine; studies in rats reported that the amounts of arginine synthesized in vivo by the kidney depend on the plasma citrulline levels (60) and low plasma citrulline was associated with low renal arginine output (215) independent on the renal capacity to synthesize arginine. Furthermore, low arginine levels were suggested to be the driving force for arginine synthesis by the kidney (157). Arginine synthesized by the kidneys is released into the bloodstream and taken up by tissues for protein synthesis or arginine degradation by the NOS or arginase pathway (see also: 1.7 Arginine requirements).

Muscle Depending on the circumstances, glutamine is primarily synthesized in skeletal muscle. Muscle therefore, is a major endogenous source of glutamine, which, via ornithine, serves as main provider for the citrulline synthesis by the intestine (15). This citrulline essentially serves as the precursor for arginine synthesis in the kidney. In the anabolic state, muscle is a net arginine consumer. Arginine in skeletal muscle can be incorporated into de novo synthesized protein (206). Arginine-enriched feeding solutions induce a marked increase in muscle glutamine concentration and enhance muscle protein synthesis in tumor- bearing rats (146). Arginine, via the OAT enzyme provides glutamate. Glutamate is a common substrate of glutamine but is also an amino donor involved in the transamination of pyruvate subsequently increasing intramuscular glutamine and alanine concentration, respectively (175). Another fate of arginine in muscle is its degradation. Cultured skeletal muscle cells contain a high arginase capacity responsible for ornithine and urea synthesis from arginine (151). However, under fasting or stress conditions, the muscle becomes catabolic and releases arginine from protein 
breakdown. Under catabolic conditions protein degradation from skeletal muscle may become a major source of arginine when compared to renal de novo synthesis (15).

\subsection{Arginine requirements}

As already mentioned in the introduction of this chapter, arginine is an amino acid dispensability of which depends on the circumstances and which is considered a semiessential amino acid in growth (92) and stress conditions (192). Arginine requirements also depend on species-related enzyme distributions. Dietary arginine is required for young growing species like dogs, cats, rabbits, guinea pigs, rats and pigs (11) because the endogenous arginine biosynthesis is not capable to meet the arginine need. In humans with arginine-restricted diets, intestinal citrulline production from glutamine is sufficient to provide enough arginine to sustain growth (196), although not enough for optimal growth (11). Not only in growth but also in other settings of increased demand such as metabolic stress, the requirements for arginine in protein synthesis and other processes will increase as extensively discussed in paragraph 3: Arginine and $\mathrm{NO}$ in trauma and sepsis.

\subsection{Arginine homeostasis}

Whole body arginine production is achieved by breakdown of dietary protein intake, endogenous production from protein degradation, and endogenous de novo production. By synthesizing arginine, the kidney plays a major role in the maintenance of arginine homeostasis. In the pig, besides the argninine synthesizing capacity the kidney, the anabolic process of intestinal arginine synthesis may be an additional way of maintaining constant arginine levels under circumstances when the availability of arginine from renal production becomes limited. Under prolonged catabolic conditions more protein is degraded than synthesized. As a consequence, more arginine appears in the circulation than disappears by utilization in protein synthesis, which provides a means to replenish the arginine plasma pool. In response to starvation and low arginine levels, the kidney is capable of upregulating the arginine synthesizing enzymes ASS and ASL as an adaptive response to maintain plasma arginine levels in response to starvation and low arginine levels (132). Although the rate of citrulline to arginine transfer was initially thought to depend on arginine levels (132), Castillo et al. postulated that the rate of arginine de novo synthesized was independent of arginine content in the diet (32). Maintenance of plasma arginine levels rather depended on a decrease in the rate of arginine degradation than on an increase in the de novo synthesis of arginine (34). After liver transplantation for OTC deficiency, plasma citrulline levels still remain low, whereas after transplantation for ASS deficiency, plasma citrulline levels remain high and arginine levels low (159). 
These findings indicate that endogenous routes for arginine production cannot fully compensate arginine deficiency.

Physiological interactions and relationships between the pathways of arginine synthesis and catabolism are complex and difficult to analyze, owing to changes in expression during development and in response to diet, hormones and cytokines. The ongoing development of new cell lines and animal models using cDNA clones and genes for key arginine enzymes will provide new approaches more clearly elucidating the physiological roles of these enzymes.

\section{Sepsis}

The septic process is characterized by serveral critical stages. Sepsis has been defined as "the systemic inflammatory response to infection" (25). The clinical syndrome includes fever, leukocytosis, elevated cardiac output, and reduced systemic vascular resistance. Severe sepsis is defined as "sepsis associated with organ dysfunction, hypoperfusion abnormality, or sepsis-induced hypotension". Sepsis-induced hypotension is defined by the presence of a systolic blood pressure of less than $90 \mathrm{~mm} \mathrm{Hg}$ or its reduction by 40 $\mathrm{mm} \mathrm{Hg}$ or more from baseline in the absence of other causes for hypotension (eg, cardiogenic shock). Vascular leak, decreased tension and systemic volume can lead to irreversible septic shock. Septic shock is a subset of severe sepsis and is defined as sepsis-induced hypotension, persisting despite adequate fluid resuscitation, along with the presence of hypoperfusion abnormalities or organ dysfunction

\subsection{Clinical aspects of sepsis}

Septic shock is the most common cause of death in the intensive care unit and causes more than 40.000 deaths per year in the USA (1). In the Netherlands the incidence of the sepsis syndrome was 13.6 per 1000 patients admitted (all hospitalized patients) while the incidence of septic shock was 4.6 per 1000 (105). Mortality in septic shock amounts to approximate 40 to $70 \%$ despite aggressive treatment $(72,201)$. Recent clinical trials that were directed against the mediators underlying sepsis did not demonstrate an improvement in survival (137). At present, there is a continuing search for new therapeutic applications and one potential target of interest is the manipulation of the production of NO.

\subsection{Gram-negative sepsis}

Bacteria causing sepsis are mainly the gram-negative, which possess endotoxin and are able to induce endotoxic and septic shock. Endotoxins constitute the major toxic 
component of the cell wall of all gram-negative bacteria. This macromolecular glycolipid is termed lipopolysaccharide (LPS) and is responsible for many of the pathologic effects of these microorganisms. Endotoxins of gram-negative bacteria provide the key signal in the immune response manifest during inflammation and sepsis. Gram-negative bacteria gain access to the bloodstream by evading host defense in immunocompromised hosts. LPS binds to monocytes and macrophages by specific interaction via receptors such as scavenger receptors CD14 and CD18, and by non-specific interactions, and enter the cells via receptor- mediated endocytosis, absorptive pinocytosis, phagocytosis, and diffusion (103). The interactions of LPS with monocytes and macrophages trigger a broad spectrum of cellular responses, including production of important mediators, such as interleukin-1 (IL-1), tumor necrotising factor (TNF), interferons (IFN), prostaglandins $(P G)$, and macrophage-derived growth factor, which are implicated in the pathogenesis of septic shock (103). Macrophage derived cytokines induce an array of responses, including fever to defend against the invading bacteria, tissue damage and activation of hormone production, thereby affecting organ and whole body metabolism (122).

\subsection{Clinical symptoms of sepsis}

Two different phases are distinguished in shock: an initial hypodynamic phase and a later hyperdynamic phase. In the older literature endotoxin shock was generally considered to be hypodynamic. In the intensive care, patients are generally with hyperdynamic shock for two reasons, firstly because patients are generally admitted after the initial hypodynamic shock phase is already over, secondly because all patients are resuscitated with parenteral fluids, allowing the circulation to become hyperdynamic. Similarly in experimental animals subjected to endotoxin a hyperdynamic circulation develops provided the animals are also resuscitated with fluid and electrolytes. The clinical picture accompanying this hyperdynamic phase consists of the following features: (a) Tissue edema, resulting from vasodilatation (decreased peripheral resistance) and increased capillary leakage. This implies that more fluid, plasma protein, leukocytes, macrophages and electrolytes leave the vascular compartment and accumulate in the tissues, and most outspokenly in the area of the most damaged tissues. The mechanisms underlying these phenomena are poorly understood. Increased capillary leakage is most likely mediated by cytokines (for instance TNF), The necessity to deliver cells and proteins to the wounded area, operative in the healing response, may be served by this process. (b) The vasodilatation described implies that intravascular volume increases, which induces shock if no adequate resuscitation is achieved. Both intravascular volume and extravascular extracellular volume increase, whereas intracellular volume decreases. The loss of intracellular volume furnishes part of the volume necessary to increase intravascular and extravascular extracellular volume and 
suffices when the stress and consequentily the stress response is modest and shortlived. (c) The hyperdynamic state is also characterized by increased cardiac output, which is induced by several factors. When intravascular volume is replenished by fluid resuscitation the heart increases its output to keep up blood pressure. Energy expenditure, oxygen uptake and substrate utilization are increased during the stress response, which requires either increased delivery of oxygen and substrate. In the stressed state, the increased cardiac output may be a tool to compensate for the typically impaired fractional extraction of oxygen and substrate by increasing blood flow to the organs.

\subsection{Metabolic aspects of sepsis}

Many parallels can be drawn between the metabolic profile of the severely injured and septic patient. The response following injury and sepsis is divided into a period of shortterm alterations, "the ebb-phase" or "shock-phase". The ebb-phase corresponds to a period of hypovolemia and sympathic activity. The following more prolonged phase, the "flow-phase", is characterized by a "hyperdynamic phase" with increased cardiac output peripheral vasodilation and a decreased ability of the tissues to extract oxygen and metabolites. Many metabolic abnormalities characterize this so called flow phase including muscle wasting and increased synthesis of acute phase proteins, ultimately leading to a negative nitrogen balance $(29,37)$. The synthesis of the acute phase proteins like $\mathrm{C}$ reactive protein (CRP), fibrinogen and ceruloplasmin rises, which are therefore called positive acute phase proteins, and the concentration of proteins like albumin, transferrin and many others drop which are for that reason called negative acute phase proteins. The relative amount of albumin of total liver protein constitutes about $52-65 \%$ and of the other liver acute phase proteins ( $\alpha$ - and $\beta$-globulins) about 19$33 \%$. The increased release of hormones mediate many of the metabolic and pathophysiological changes seen in sepsis and trauma. There is also increasing evidence that cytokines released in response to tissue injury and/or microbial invasion are involved in the alterations in metabolism occurring in severe sepsis (184). Trauma and sepsis also initiate changes in carbohydrate and fat metabolism (see below) $(35,37$, 174).

Hormones and cytokines Stress hormones are potential mediators of the deranged metabolism observed in trauma and sepsis (115). Hormones, particularly cortisol, catecholamines and glucagon induced characteristic changes in the muscle and plasma amino acid patterns similar to those seen early in protein catabolism $(53,83,124)$. The hormone-mediated mobilization of amino acids and the production of glucose provide energy for host immune response and wound repair (124). The sepsis-induced hormone 
secretion is a principal reason for the increased metabolic rate and nitrogen loss during stress. It is now clear that the acute phase response is not only regulated through hormonal mediators, but also by inflammatory mediators that act in concert to produce the manifestations of the hypermetabolic state seen in stress. TNF- $\alpha$ and IL-6 are two major key cytokines involved in the generation of the acute phase response in sepsis. IL6 correlates well with the severity of sepsis or injury and is responsible for the fever and metabolic changes in the acute phase. TNF- $\alpha$ has been proven to be the mediator that orchestrates the hemodynamic changes and tissue injury in septic shock.

Carbohydrate It is generally agreed that in septic patients glucose turnover is increased. Changes in carbohydrate metabolism after severe trauma (37) and during sepsis (37) or endotoxemia (178) include hyperglycemia resulting in glycolytically produced hyperlactacidemia. Peripheral insulin resistance that is a common phenomenon in sepsis, results in prioritization of glucose flux to the predominantly glucose-consuming cells, such as the wound, the inflammatory, and immune cells, all insulin-independent cells $(37,53)$. An elevated supply of gluconeogenic precursors to the liver accelerates gluconeogenesis, which is primarily responsible for the increased whole body glucose production, after hepatic glycogen stores are depleted (178). Lactate release from skeletal muscle is enhanced, due to the more sustained activation of muscle glycolytic activity and increased uptake of plasma glucose (178). Peripheral insulin resistance and increased muscle glucose utilization under septic conditions is contrasting. 5'AMP-activated protein kinase (AMPK) can be activated in response to cellular fuel depletion and is a central mediator of insulin-independent glucose transport enabling fuel-depleted muscle cells to take up glucose under conditions of metabolic stress (86). Stimulation of the immunologically active tissues by endotoxin with the participation of mononuclear phagocytes results in elevated glycolysis in these tissues as well, thus further enhancing the hyperlactacidemia. Hyperlactacidemia is a frequent manifestation in the response to hypodynamic sepsis and is considered a prognostic indicator of illness severity in septic patients (155). An increased flow response in a condition of hyperdynamic sepsis is associated with enhanced glucose production by the liver $(20,54)$, and increased glucose clearance by increased glucose transport by macrophage-rich tissues, including the spleen and ileum (128). Complications seen after endotoxin shock seem often related to the rate of fluid resuscitation, and thus, repletion of plasma volume. If additional fluid resuscitation is administered, a hyperdynamic state with elevated cardiac output, organ perfusion and metabolic rate, associated with increased glucose turnover, are achieved.

Protein A hallmark of the septic response is the rapid depletion of muscle mass. The negative protein balance is manifested by a massive excretion of urea $(8,129,162)$. 
Nitrogen losses of $40 \mathrm{~g}$ nitrogen a day ( $20 \%$ of body protein) are observed during severe sepsis (113). Mobilization of amino acids from skeletal muscle results from increased protein breakdown and/or decreased protein synthesis. The relative contribution of protein degradation depends on the severity of the septic insult (189). The recent view is that muscle breakdown furnishes essential substrates for wound healing and host response. When the septic response becomes chronic as is sometimes the case in patients in the ICU with unresolved or not completely resolved sepsis, this muscle breakdown leads to severe muscle depletion. The clinical implication of this continued muscle protein loss is an inadequate host response and loss of muscle strength (42, 204). Endocrine hormones, e.g., cortisol and glucagon are largely responsible for these effects. Endotoxin-induced cytokines, including IL-1, IL-6, TNF- $\alpha$ and IFN $\beta_{2}$ also play an important regulatory role in protein synthesis and degradation and amino acid fluxes during sepsis (68). IL-1 is thought to be one of the cytokines involved in increased branched chain amino acid (BCAA) catabolism in muscle (140). In addition to cytokines and hormones, NO production that results from induced NOS-II activity, probably also plays a role in the alterations in protein metabolism observed in sepsis (see also: 1.5 Involvement of arginine and its metabolites in metabolism).

\subsection{Inter-organ metabolism during sepsis}

Trauma (176) and sepsis (189) alter the flux of metabolic substrates between skeletal muscle and liver. It is also increasingly recognized that the simultaneous synthesis and degradation of proteins is important in the regulation and adaptation to changing environments. The turnover of individual proteins may change in opposite directions, thereby leading to fluxes of alpha-amino-nitrogen that serves to adapt to and survive during stress conditions. The metabolic response to sepsis is associated with the rapid net breakdown of the body's muscle protein and glycogen stores leading to shifts in the inter-organ substrate fluxes. Increased efflux of amino acids from increased breakdown of peripheral tissue (i.e. skeletal muscle) to splanchnic tissue (i.e. intestinal tract plus liver) serve to provide substrate for the liver and other visceral organs like the gut and the spleen (176). The transamination of the branched-chain amino acids (BCAA), leucine, isoleucine and valine into branched-chain $\alpha$-keto acids (BCKA) occurs almost exclusively with $\alpha$-ketoglutarate to form glutamate (Figure 4 ). The three different BCKA products yield three different glucogenic and ketogenic CoA derivates. Glutamate may donate its amino group to pyruvate to form alanine or may incorporate free ammonia to form glutamine. 


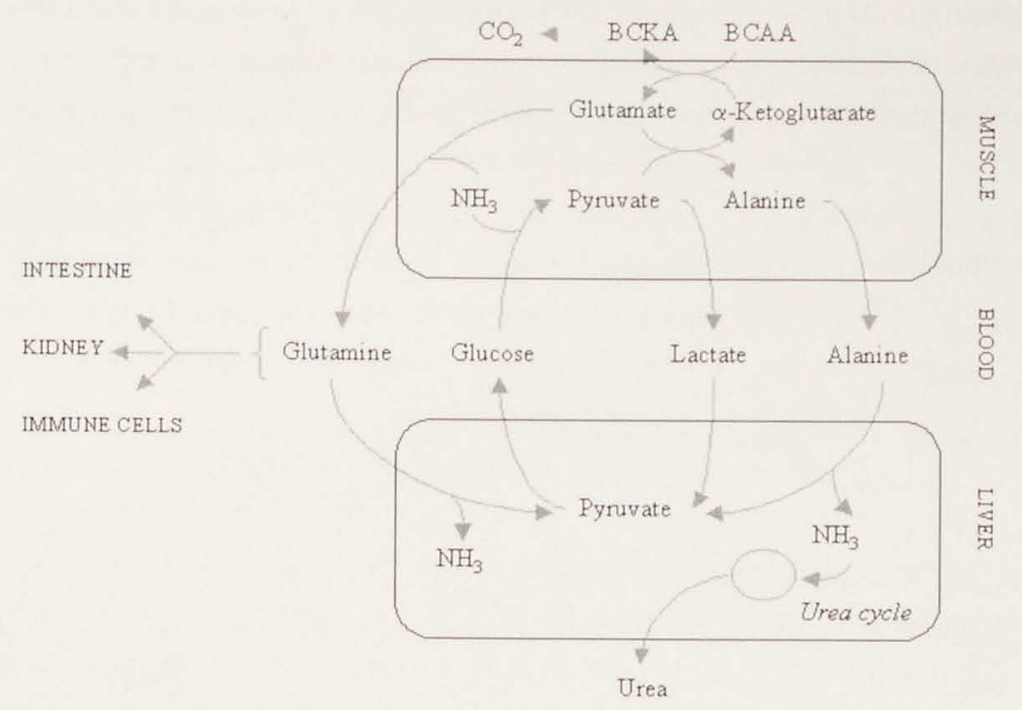

Figure 4 . Scheme of the carbon and nitrogen cycling between the different organs.

An increase in this transamination and subsequent nitrogen efflux from muscle during sepsis foresees in the increased substrate demand by immune cells, intestines and kidneys during septicemia (90). Not only alanine is de novo synthesized from pyruvate in muscle, but also lactate (46). Lactate release from skeletal muscle and other organs is enhanced due to increased utilization of plasma glucose and sustained activation of glycolysis (178). In the liver, the carbon skeletons of the muscle-derived amino acids and lactate in the liver serve as precursors for pyruvate, which is used for glucose production by gluconeogenesis (20). The amino groups of the amino acids are processed into urea by means of the ornithine or urea cycle (90). Because of increased supply of gluconeogenic precursors, the gluconeogenesis is elevated during sepsis (20). Similarly, Increased nitrogen supply from amino acid and ammonia uptake by the liver is followed by enhanced urea synthesis (20). The subsequent glucose production is thought to meet increased glucose requirements of peripheral tissues and by the immune system (128). During sepsis, lactate, alanine, glutamine and additional amino acids are released in an increasing extent from muscle protein resulting from degradation and de novo synthesis yielding substrate for liver gluconeogenesis, for oxidation, energy production and for protein synthesis in the different organs. These gluconeogenic precursors together with glucose and BCAA, therefore, play an important role in this peripheral-central axis. The carbon cycle of glucose and glutamine/alanine between peripheral and central organs is called the Felig cycle (67) whereas the carbon cycle of lactate and glucose between periphery and central organs is known as the Cori cycle (43). The existence of the Cori cycle in principle provides energy in these tissues without really having to produce new 
glucose, utilizing carbon skeletons from amino acids once glycogen stores are depleted. The drawback of the Cori cycle is that it is energetically costly in that more ATP is required to synthesize glucose in the liver than is generated by glycolysis in the periphery.

\subsection{Nutritional aspects of sepsis}

Elevated metabolism in septic patients implicates mobilization of amino acids and the production of glucose that act as precursor for the synthesis of acute phase proteins serving the acute phase response and as precursors of gluconeogenesis (124). This response is associated with hypermetabolism, a negative nitrogen balance and hyperglycemia (37). Hypermetabolism, the negative nitrogen balance and lack of spontaneous food intake provide the basis for applying nutritional support. Nevertheless, enhanced hepatic glucose production and elevated protein catabolism are difficult to suppress, even during aggressive nutrition support (37). Initially, nutritional support is often at least in part infused via the intravenous infusion route since the gastrointestinal tract of septic patients often is unable to cope with complete enteral nutrition. As soon as possible, nutritional support is added via the enteral route.

\section{Arginine and NO in trauma and sepsis}

\subsection{Induction of NOS-II during sepsis}

During sepsis and inflammation profound changes in physiological function are induced by a variety of mediators, including endotoxin and various cytokines. Many of these mediators, in addition to their other functions, induce the synthesis of NO through the induction of NOS-II within a variety of cell types. The expression and activation of NOS-II is widespread, including vascular endothelial cells, smooth muscle cells, macrophages and different parenchymal cells (141). Many mediators including endotoxin (LPS) and the pro-inflammatory cytokines $I L-1 \beta, I L-2, I L-6, T N F-\alpha$ and IFN- $\gamma$, have shown to induce NOS-II and thereby to induce an exaggerated NO production in vitro (139) and in vivo (89). Multiple cytokines are required to achieve maximal induction of NOS-II. In LPS and IFN- $\gamma$ stimulated macrophages, presence of NOS-II messenger ribonucleic acid (mRNA) is observed after $2 \mathrm{~h}$ and NOS-II protein after $4 \mathrm{~h}$. The NOS-II expression in macrophages may be present for several days after the beginning of the disease process (214). 


\subsection{Dual role of NO during sepsis}

NO has a dual role. Produced in physiological quantities, NO is a crucial mediator involved in organ functioning. Paradoxically, produced in large amounts, it also contributes to tissue injury and organ dysfunction. There are examples of chronic localized inflammation in both animal and human models which suggest that chronic NOS-II expression may be detrimental whereas the role of NO during episodes of acute inflammation appears to be a protective one. Therefore, depending on its concentration and exposure time, NO exerts either a signaling or a cytotoxic function (for extended reviews see: $(7,104,139))$.

\subsection{NO in different responses during sepsis}

Under normal physiological circumstances, NO acts as a mediator molecule in the immune response (a), in blood pressure regulation and blood flow distribution (b) and in neuronal communications (c). However, after exposure to bacterial endotoxin or certain cytokines, expression of NOS-II occurs in a wide variety of tissues producing large amounts of $\mathrm{NO}$ for long periods. The uncontrolled production of NO by the activated NOS-Il enzyme may interfere in the normal physiologic response. NO formed by NOS-II has been attributed a pivotal role in the pathophysiological alterations observed in inflammation and sepsis. NO released from NOS-II in vascular tissues may cause profound vasodilation and alterations in hemodynamics (a). Knowledge about the role of NO derived from NOS-II in neurotransmitter tissue (b) is scarce. In some cells, including macrophages, this $\mathrm{NO}$ appears to be an important mediator in immune defense (c).

(a) Hemodynamics Produced in moderate amounts, NO has proven to mediate vasodilatation by vessel relaxation $(95,150)$ in the cardiovascular system. NO released from endothelial cells acts as a diffusible molecule that, in adjacent smooth muscle, activates soluble guanylate cyclase leading to increased cyclic guanosine monophosphate (CGMP) levels which in turn produce relaxation (see figure 5 and for extended review: (11)). Sepsis-associated endotoxins and cytokines induce excess production of NO by NOS-II. The excess production of NO by NOS-II was thought to importantly contribute to the systemic hypotension observed in septic shock (107). The NO-induced mechanism of vascular smooth muscle relaxation mediates vasodilatation and vascular leakage that underlie vascular hypotension and hyporeactivity. The vascular hyporeactivity in septic chock is characterized by hyporeactivity to traditional vasopressors $(91,153)$. The ensuing compromised cardiac output and organ perfusion may lead to hypovolemia and irreversible hypovolemic shock (107). However, the hemodynamic pattern characteristically seen in patients with reversible shock has a biphasic pattern. Most experimental animal models have failed to reproduce the early, 
hyperdynamic phase of sepsis and endotoxemia, inducing profound hypodynamic responses. Experimental hyperdynamic sepsis elicited by long-term endotoxin administration and fluid support, results in a different hemodynamic profile; hyperdynamic sepsis involves increased cardiac output whereas systemic vascular resistance is abnormally low. High cardiac output and low systemic vascular resistance reflect a hypercirculatory state if left ventricular filling pressure is maintained by carefully monitored volume substitution (111). In this hyperdynamic state, especially regional blood flow to the myocardium and liver are increased (111). During episodes of shock, cardiac output tends to decrease to below the normal range while systemic vascular resistance remains abnormally low (111). Inhibition of NO production in the treatment of sepsis caused a significant decrease in blood flow to all vascular beds in vivo (88). Therefore, the benefit, if any, of the inhibition of NO in the treatment of hypovolemic shock may be useful but is questionable in hyperdynamic sepsis. Although NO may cause hypotension in septic shock, NO is crucial for the maintenance of microcirculatory blood flow and vascular integrity in hyperdynamic sepsis. NO was shown beneficial in preserving organ perfusion during acute endotoxemia in rats (150). NO inhibitors reduced the blood flow to stomach, duodenum, jejunum, ileum, cecum, and colon in portal veinstenosed rats (99) and portal flow to the liver in endotoxemic rats $(4,10)$. Arginine was found to protect against liver injury (6). These studies suggest that an increase in synthesis of NO plays an important role in the splanchnic circulation. There is also evidence in literature that arginine exerts a beneficial effect in renal disease by improving renal plasma flow (163). Therefore, NO synthesis appears to be beneficial in controlling blood flow to virtually all organs.

(b) Neurotransmission In the autonomic nervous system, NO is found in neurons, where it functions as a messenger molecule (see figure 5). In the autonomic nervous system NO formed by NOS-I functions as a major non-adrenergic non-cholinergic (NANC) neurotransmitter (181). This NANC pathway plays a particularly important role in relaxation of smooth muscle, in the cerebral circulation, in the gastrointestinal (31), urogenital and respiratory tracts (28). In the gastrointestinal tract, the coordination of peristalsis and sphincter relaxation is mediated by the release of NO. In inflammatory disease such as sepsis, bacterial endotoxin or cytokines are responsible for the induction of NOS-II activity in the gastrointestinal tract. Excess NO production was found to reduce intraluminal pressure (31) and interrupt periodic cycling of the migrating motor complexes (MMC's) $(165,168)$. It was demonstrated that NO inhibitors restored endotoxin challengeinduced disorganization of the normal motility pattern in dogs $(46,64)$, indicating a regulatory function of $\mathrm{NO}$ in the nervous system. NO also plays a major role in skeletal muscle by regulating contractility and muscle perfusion (28, 110). NO formed by NOS-I acts as a neurotransmitter in the brain and peripheral nervous system (191). NO 
produced by NOS-I in the NANC nerves of the gut, is involved in intestinal motility (181). While NOS-I derived NO normally functions as a physiological neuronal mediator in neurotransmission and motility, excess production of NO resulting from the activation of NOS-11 mediates disruption of normal functioning. Excess NO was attributed to play a major role in delayed gastric emptying $(46,206)$, paralytic ileus (185), and diarrhea (73) common after sepsis. NO seems involved in diarrhea associated with complete disruption of the MMC's and accelerated transit of gastrointestinal content (47).

(c) Immune defense

NO occupies a key position in regulation of immune function (82) thereby having a dual role by both damaging and limiting infection and tissue injury. Macrophages effect their antimicrobial activity principally through induction of NOS-II and the subsequent generation of $\mathrm{NO}$ (Figure 5 ) and oxidants $(40,44)$. The radicals that are generated by the massive release of $\mathrm{NO}$ in the cascade reaction (see also: 1.3 Arginine catabolism, nitric oxide) are responsible for much of the tissue damage associated with diverse pathophysiological conditions including inflammation. NO acts as a free radical, inherently reactive and in large amounts mediating cellular toxicity by damaging critical metabolic enzymes and by reacting with superoxide to form an even more potent oxidant, peroxynitrite. Moreover, peroxynitrite is highly cytotoxic due to its ability to oxidize sulfhydryl groups, lipids, proteins, enzymes and DNA (16). Under normal conditions, NO produced in low concentrations may be considered being cytoprotective by acting as a free radical scavenger since NO has been shown to interact with oxygen free radicals (205). Alternatively, when high amounts of NO are formed, formation of the peroxynitrite radical and "reactive nitrogen radicals" will mediate oxidative and nitrosative cytotoxicity, thereby, protecting the host from infection. In severe inflammation, oxidants may be responsible for induction of apoptosis and inhibition of cell proliferation by altering proteins and interacting with DNA synthesis (168). Suppressive effects of sustained NO activation on lymphocyte proliferation (40) contribute to a protective function of NOS-II. Sustained production of NO endows macrophages with cytostatic or cytotoxic activity against viruses, bacteria, fungi, protozoa, helminths, and tumor cells. This cytotoxic property of NO constitutes a primary mechanism of non-specific immune defense against microorganisms $(14,89)$. In addition, NO also limits the development of tissue damage by inhibiting platelet leucocyte adhesion and/or aggregation within postcapillary venules (112). NO prevents epithelial permeability by the inhibition of the mast cell activation (101). There is also indication that NO inhibits tumor growth by tumor cell killing (164). This effect may, at least in part, be mediated by immunologic effects. An increasing number of studies show the detrimental effect of NO-inhibitors in sepsis and information is now accumulating on the beneficial role of induced NO in organ function and tissue defense (for extended review see: (135)). 

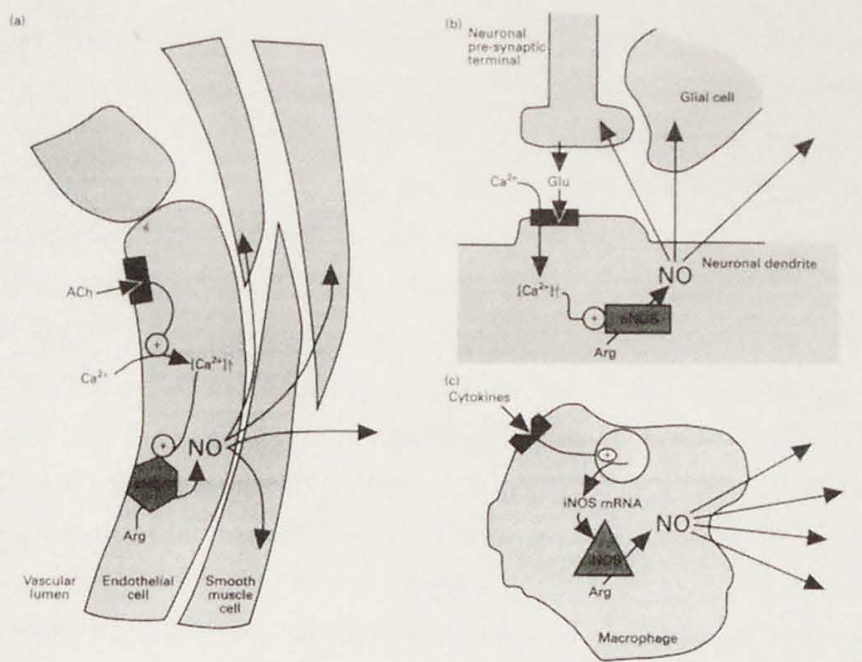

Figure 5. NO synhtesis by the known NO synhtase isoenzymes. Three examples of NO synthesis are shown: (a) synthesis of NO by NOS-III in a vascular endothelial cell stimulated by acetylcholine (Ach); (b) NO synthesis by NOS-I in a neuronal dendrite stimulated by glutamate (Glu); and (c) NO synthesis by NOS-II in a macrophage following induction of NOS-II mRNA and enzymes by cytokines. Induction of NOS-II may also interfere in the (a) and (b) pathways.

\subsection{Inhibition of NO}

Overproduction of NO plays an important role during sepsis. Some strategies to maintain blood pressure and improve perfusion in septic shock in sepsis have aimed at NO considering its vasodilator, immune modulatory, and in high concentrations, cytotoxic effect (138), inhibition of its synthesis or activity is therefore considered to be potentially beneficial as a therapeutic approach in septic shock. Three ways of inhibition can be distinguished. Firstly, by inhibiting NOS-II activity with NOS-II antagonists that inhibit NOS-II activity (NOS-II inhibitors) or by inhibiting the second messengers (i.e., cGMP) in the NOS cascade (75). Secondly, by modifying the balance between pro-inflammatory and anti-inflammatory cytokines by selective antibodies. Another novel way to inhibit NO synthesis is to inhibit the NOS-II cofactors, such as $\mathrm{BH} 4$ although this has not been tested in experimental shock (30).

NOS inhibitors result in reduced levels of cGMP in vascular muscle cells and in cardiomyocytes and therefore are used to treat hypotension and cardiac dysfunction in septic shock. Initially, experiments with NOS-II inhibitors or NOS-II knock-out mice suggested that the inhibition of NO synthesis might be beneficial during sepsis. An increasing number of experiments have revealed that inhibition with non-selective inhibitors had either no effect or in fact may be harmful (152) and may aggravate the hypotension and vasodilatation during sepsis (for thorough review see: (107)). Inhibition 
of systemic NO decreases hepatic and renal blood flow and reduces tissue perfusion, which may result in tissue damage. NO inhibition may also limit the ability to control infections and, thereby, counteract the potentially beneficial effects on the immunedefense system. Limitations of NO production during sepsis may not only compromise the cardiovascular and immune system, but also NO-mediated neurotransmission. Most animal studies and all human studies have studied the effects of non-specific NOS inhibition. This is a potential weakness because both constitutive NOS (NOS-I and III) and NOS-II are blocked by this method including the "signaling" NO formed by constitutive NOS. Nevertheless, NOS-II inhibitors also had no beneficial effect on hemodynamics and mortality (195). The divergent results from experimental studies with inhibitors may be explained by differences between 1 . animal models used, 2 . the type (selective vs. non-selective), dose and way of administration (continuous infusion vs. bolus injection) of NOS inhibitors, 3. the moment at which NOS inhibitors have been administered, and 4. may reject the hypothesis that part of the NOS-II related NO production is an "overshoot" and harmful to the organism.

So far, none of the inhibitors have been developed for pharmacological use, due to undesirable side effects related to their inhibitory action on CNOS.

\subsection{Promotion of NO}

Dependency of NOS on arginine

Formation of NO by NOS-II is linearly dependent on the extracellular arginine substrate concentration. Several studies showed that extracellular arginine concentration-dependently determines the rate of NO production. Arginine deficiency was found to reduce NO synthesis by NOS-II in young rats (209). Moreover, the nitrite production by NOS-II appeared dependent on the arginine substrate availability since maximal production rates were reached by arginine concentrations of $500-1000 \mu \mathrm{M}(23,80)$. Availability of extracellular arginine to intracellular NOS-II, therefore, is a critical step in controlling NO production. Many cell types, including endothelial cells (87), smooth muscle cells (84) and macrophages (144), contain the enzymes ASS and ASL and therefore have the capacity to synthesize arginine from citrulline, providing intracellular substrate for the production of NO. ASS and ASL are induced in many tissues by LPS treatment in rats (136). In principle, such an arginine-citrulline cycle can make cellular synthesis of NO independent of extracellular arginine. Moreover, the capacity for arginine transport was found to increase by inflammatory agents in different cells including macrophages $(23,102,173)$ and hepatocytes (148). The apparent $K_{m}$ (substrate concentration at which the transport rate is one half maximum) of extracellular arginine for intracellular NO synthesis is approximately $73-150 \mu \mathrm{M}(147,211)$. The $K_{m}$ values of the $y+$ system that mediates 
arginine transport ranges from 100-150 $\mu \mathrm{M}$. This is within the physiological concentration range of arginine (100-110 $\mu \mathrm{M}$ under post-absorptive conditions and 155-165 $\mu \mathrm{M}$ under fed conditions in the pig). Normally intracellular arginine concentrations (0.1-1.0 mM) greatly exceed the $K_{m}$ of NOS for arginine and should be sufficient to saturate NOS. Therefore, formation of NO by NOS-II under physiologic conditions is not critically dependent on extracellular arginine. However, under conditions of sustained NOS-II activity such as fasting and severe stress such as sepsis, extracellular arginine may become depleted. Arginine uptake can become rate-limiting $(63,117,142,172)$ to sustain maximal rates of NO synthesis by NOS-11 expressing cells following endotoxin and cytokine exposure. Moreover, evidence suggests that ASS activity is rate-limiting to highoutput NO synthesis (213).

Decreased arginine availability The metabolic response to stress conditions such as severe and chronic infection (sepsis) is characterized by decreased plasma levels of glutamine and arginine (123). In animals and humans with sepsis, plasma arginine levels were found to be markedly decreased suggesting compromised endogenous synthesis and/or increased utilization of arginine (71). In addition, dietary arginine sources are often low during trauma or sepsis because of impaired dietary intake. During sepsis, peripheral protein degradation is accelerated resulting in increased release of arginine into the circulation. This muscle arginine release serves an important role in the replenishment of plasma levels of arginine and, therefore, in the maintenance of arginine homeostasis. Sustained net protein degradation, however, results in continued net loss of body protein, which may, on the long-term, potentially lead to depletion of the plasma arginine pool. Considering the kidneys are a main source of the de novo arginine synthesis, reduced glutamine and glutamate availability may also contribute to reduced arginine levels via impaired intestinal citrulline production and subsequent compromised renal arginine production (203). The intestinal capacity for citrulline production appears to determine the renal biosynthetic capacity for arginine synthesis in a concentration dependent-manner (see also: 1.6 Organ characteristics of arginine metabolism, kidney) (60). In rats, partial enterectomy caused a reduction in intestinal glutamine uptake and citrulline release by approximately $60 \%$ and a reduction in the arginine levels by approximately $40 \%$ (58). Intestinal citrulline production, therefore, appears to be the rate-limiting step in the synthesis of arginine.

In conditions of prolonged disease, arginine availability from endogenous production and arginine regeneration mechanisms may not be sufficient to meet the increased requirements for different processes. Sites of inflammation with prominent macrophage infiltration, such as wounds and certain tumors, were found deficient in free arginine (3, 
$80,84,112)$. For that reason, administration of additional exogenous arginine may be of benefit by means of overcoming the significantly increased requirements.

Arginine as a therapeutic modality

By serving as a precursor for NO, polyamines and other molecules with biological importance, arginine plays versatile key roles in nutrition and metabolism. Moreover, arginine is the most abundant carrier of nitrogen, partly because this amino acid contains four nitrogen atoms per molecule. Arginine administration was found beneficial in improving many organ functions. Arginine is involved in facilitating wound healing, synthesis of reparative tissue protein, cell proliferation, blood flow regulation and immune processes. Under septic conditions, induced NOS-II that becomes activated by bacterial products and cytokines activity will increase the requirements for arginine for the production of $N O(85,117)$. The induction of NOS-II increases the demand for arginine in diverse cell types such as vascular endothelial cells, macrophages and hepatocytes that can respond to cytokines by upregulating their arginine transport (for extensive discussion see: "Dependency of NOS on arginine"). In animals and humans with severe stress such as sepsis, arginine pools may become compromised and endogenous arginine production become rate limiting to foresee in NO production. This provides a basis for the concept of arginine deficiency (absolute or relative) in certain settings in which arginine supplementation to the diet may be beneficial.

Addition of arginine in the diet has been shown to reduce or improve impaired nitrogen balance and weight loss in traumatized rats $(38,45,158)$ and patients $(198)$. Intravenous infusion of arginine in traumatized rats (146) also showed a reduction in urinary nitrogen excretion. Administration of enteral formulas that are enriched with multiple supplements including arginine was associated with reduced nitrogen losses and improved immune response in septic and traumatized patients $(26,27,55,198)$. Beneficial effect of additional dietary arginine on collagen synthesis in wound healing was demonstrated in rats $(12,38)$. Dietary intervention with arginine exerted beneficial effects in a number of experimental renal diseases by amelioration of renal plasma flow (163). Clinical studies using arginine-enriched diets suggest that in stress situations, these arginine formulas can be of significant benefit for injured patients. Some of these trials demonstrated improved clinical outcome by reducing postoperative infections, negative nitrogen balance and by improving gut function $(26,55)$.

In view of the fact that basic levels of arginine are crucial for maintenance of NO production, it could be hypothesized that treatment with arginine under circumstances of prolonged sepsis might be beneficial by sustaining increased requirements. Supplementation of the diet with this amino acid may be a therapeutic modality in 
overcoming NO deficiency, and by this means, be beneficial in improving defense mechanisms, impaired motility function and impaired organ perfusion associated with septic shock. In view of the fact that the therapeutic effects of NO appear to depend on the quantities formed, the timing of manipulation of NO production by increasing exogenous L-arginine intake levels is critical. Additional studies are required to delineate the role of NO in sepsis and the therapeutic benefits of modulating NO synthesis in sepsis. We hypothesized that exogenous L-arginine administration would exert no effect before the expected induction of the NOS-II enzyme activity (211). L-Arginine administration during the main expression of NOS-II might possibly induce excessive hypotension and irreversible shock (for extensive review see: (107)). Therefore, Larginine supplementation was started 8 hours after the initiation of endotoxin infusion to prevent an overproduction of NO to such an extent that it might become harmful.

\subsection{Outline of the thesis}

As can be concluded from paragraph 1, arginine plays an important role in sepsis. This study was performed to improve our understanding of the requirements for arginine in relation to the NO production during endotoxin-induced sepsis. In view of the fact that the septic condition is mostly accompanied by fasting and, hence, dietary sources of arginine are reduced, the de novo synthesis of arginine may become insufficient to meet the arginine requirements for sustained NOS-II-mediated NO production. Initially, protein metabolism, arginine-NO metabolism and the jejunal motility pattern are studied during and after sepsis to obtain more insight in the alterations evoked by endotoxin challenge. Subsequently, arginine was administrated during and after sepsis to assess the effects of providing extra NO substrate on mentioned parameters. Studies were performed in an experimental model of sepsis as will be described in chapter 2 in which the clinical condition of hyperdynamic fluid-resuscitated sepsis and enterally fed post-sepsis was imitated.

Arginine metabolism is closely related to protein metabolism. Therefore, first (in chapter 3 ), insight was obtained in the immediate effects of endotoxemia on alterations in whole body and organ protein synthesis and degradation under fasting conditions. The exact manner in which protein metabolism of the organs is changed in terms of modifications in synthesis and degradation, remains to be elucidated. In addition, changes in urea, amino acids, lactate and glucose organ fluxes were investigated in relation to whole body changes. The postponed effect of endotoxin challenge on organ and whole body protein metabolism is relatively unknown. In clinical practice, providing enteral nutritional support to critically ill patients is extremely important to improve recovery of these patients. Therefore, in the $4^{\text {th }}$ chapter, the long-term effects of the effects of endotoxin challenge 
on whole body and organ protein turnover and inter-organ fluxes are investigated during nutritional support. Chapter 5 describes the effects of arginine, intravenously infused during endotoxemia and intragastrically infused following endotoxemia upon the whole body and organ protein metabolism. Chapter 6 outlines the effect of endotoxemia on NO synthesis by the different organs in relation to their arginine turnover. The effects of intravenous arginine supplementation during endotoxemia and intragastric arginine supplementation after endotoxemia on the whole body and organ NO production will be outlined in chapter 7. Side effects of arginine administration under endotoxemic conditions on urea-cycle fluxes and intermediate metabolism are described in chapter 8. Motility readings in endotoxemic pigs were performed by manometry as functional parameter of sepsis in view of the fact that arginine may be involved in motility through intestinal NO production. The short and long-term effects of endotoxemia on the motility pattern are presented in chapter 9 and put into relation to visceral NO production. Effects of arginine infusion during and after endotoxin treatment on the jejunal motility pattern and visceral NO production are presented in chapter 10. The results of the thesis are discussed in chapter 11 and summarized in chapter 12.

\section{References}

1. Mortality patterns - United States 1992. MMWR Mort Mental Wkly Rep 43: 916-920, 1994.

2. Akesson, B., H. Mosen, G. Panagiotidis, and I. Lundquist. Interaction of the islet nitric oxide system with L-arginine-induced secretion of insulin and glucagon in mice. $\mathrm{Br} J$ Pharmacol 119: 758-64, 1996.

3. Albina, J., M. Caldwell, W. Henry, and C. Mills. Regulation of macrophage functions by $\mathrm{L}$ arginine. Journal of Experimental Medicine 169: 1021-1029, 1989.

4. Albornoz, L., J. C. Bandi, S. Sanchez, E. Spinedi, G. Canteros, J. A. De Paula, O. Galdame, and R. Mastai. [Role of nitric oxide in alterations of the systemic and splanchnic hemodynamics in a experimental model of portal hypertension]. Medicina 54: 17-24, 1994.

5. Anasuya, A., and B. S. Rao. Relationship between body collagen and urinary hydroxyproline excretion in young rats fed on a low-protein or low-calorie diet. Br J Nutr 24: 97$107,1970$.

6. Angele, M. K., F. Fitzal, N. Smail, M. W. Knoferl, M. G. Schwacha, A. Ayala, P. Wang, and I. H. Chaudry. L-arginine attenuates traumahemorrhage-induced liver injury. Crit Care Med 28: 3242-8., 2000.
7. Änggård, E. Nitric oxide: mediator, murderer, and medicine. The Lancet 343: 1199-1206, 1994.

8. Arnold, J., I. T. Campbell, T. A. Samuels, J. C. Devlin, C. J. Green, L. J. Hipkin, I. A. MacDonald, C. M. Scrimgeour, K. Smith, and M. $J$. Rennie. Increased whole body protein breakdown predominates over increased whole body protein synthesis in multiple organ failure. Clin Sci (Colch) 84: 655-61, 1993.

9. Ayuk, P. T., C. P. Sibley, P. Donnai, S. D'Souza, and J. D. Glazier. Development and polarization of cationic amino acid transporters and regulators in the human placenta. Am J Physiol Cell Physiol 278: C1162-C1171, 2000.

10. Ayuse, T., N. Brienza, J. P. Revelly, J. K. Boitnott, and J. L. Robotham. Role of nitric oxide in porcine liver circulation under normal and endotoxemic conditions. J Appl Physiol 78: 1319-29, 1995.

11. Barbul, A. Arginine: biochemistry, physiology, and therapeutic implications. JPEN J Parenter Enteral Nutr 10: 227-238, 1986.

12. Barbul, A., S. A. Lazarou, D. T. Efron, H. L. Wasserkrug, and $G$. Efron. Arginine enhances wound healing and lymphocyte immune responses in humans. Surgery 108: 331-6; discussion 336-7, 1990. 
13. Barroso, J. B., A. Carreras, F. J. Esteban, M. A. Peinado, E. Martinez-Lara, R. Valderrama, A. Jimenez, J. Rodrigo, and J. A. Lupianez. Molecular and kinetic characterization and cell type location of inducible nitric oxide synthase in fish. Am J Physiol Regul Integr Comp Physiol 279: R650-6, 2000.

14. Bastian, N. R., and J. B. Hibbs, Jr. Assembly and regulation of NADPH oxidase and nitric oxide synthase. Curr Opin Immunol 6: 131-9, 1994.

15. Beaumier, L., L. Castillo, Y. M. Yu, A. M. Ajami, and $V . R$. Young. Arginine: new and exciting developments for an "old" amino acid. Biomed Environmental Sci 9: 296-315, 1996.

16. Beckman, J. S., T. W. Beckman, J. Chen, P. A. Marshall, and B. A. Freeman. Apparent hydroxyl radical production by peroxynitrite: implications for endothelial injury from nitric oxide and superoxide. Proc Natl Acad Sci USA 87: 1620 1624, 1990

17. Beckman, J. S., and W. H. Koppenol. Nitric oxide, superoxide, and peroxynitrite: the good, the bad, and the ugly. Am J Physiol 271: C1424C1437, 1996

18. Beckmann, J. S., Y. Z. Ye, P. G. Anderson, J. Chen, M. A. Accavitti, M. M. Tarpey, and C. R. White. Extensive nitration of protein tyrosines in human atherosclerosis detected by immunohistochemistry. Biol Chem Hoppo-Seyler 375: 81-88, 1994.

19. Bedard, S., B. Marcotte, and A. Marette. Cytokines modulate glucose transport in skeletal muscle by inducing the expression of inducible nitric oxide synthase. Biochem $J$ 325: 487-93, 1997.

20. Beisel, W. R., and R. W. Wannemacher. Gluconeogenesis, ureagenesis, and ketogenesis during sepsis. J Parenter Enteral Nutr 4: 277 285, 1980.

21. Besset, A., A. Bonardet, G. Rondouin, B Descomps, and $P$. Passouant. Increase in sleep related $\mathrm{GH}$ and $\mathrm{Prl}$ secretion after chronic arginine aspartate administration in man. Acta Endocrinol (Copenh) 99: 18-23, 1982.

22. Blachier, F., H. M'Rabet-Touil, L. Posho, B. Darcey-Vrillon, and P.-H. Duée. Intestinal arginine metabolism during development. Evidence for the novo synthesis of L-arginine in newborn pig enterocytes. Eur $J$ Biochem 216: 109-117, 1993.

23. Bogle, R. G., A. R. Baydoun, J. D. Pearson, S. Moncada, and G. E. Mann. L-Arginine transport is increased in macrophage generating nitric oxide. Biochem J 284: 15-18, 1992.

24. Bohley, P., J. Kopitz, G. Adam, B. Rist, F. von Appen, and S. Urban. Post-translational arginylation and intracellular proteolysis. Biomed Biochim Acta 50: 343-6, 1991.
25. Bone, R. C., R. A. Balk, F. B. Cerra, R. P. Dellinger, A. M. Fein, W. A. Knaus, R. M. Schein, and $W$. J. Sibbald. Definitions for sepsis and organ failure and guidelines for the use of innovative therapies in sepsis. The ACCP/SCCM Consensus Conference Committee. American College of Chest Physicians/Society of Critical Care Medicine. Chest 101: 1644-55, 1992.

26. Braga, M., L. Gianotti, A. Cestari, A. Vignali, F. Pellagatta, A. Dolci, and V. Di Carlo. Gut function and immune and inflammatory responses in patients perioperatively fed with supplemented enteral formulas. Arch Surg 131: 1257-1265, 1996.

27. Braga, M., L. Gianotti, G. Radaelli, A. Vignali, G. Mari, O. Gentilini, and V. Di Carlo. Perioperative immunonutrition in patients undergoing cancer surgery: results of a randomized double-blind phase 3 trial. Arch Surg 134: 428-33, 1999.

28. Bredt, D. S. Endogenous nitric oxide synthesis: biological functions and pathophysiology. Free Radic Res 31: 577-96, 1999.

29. Breznock, E. M. The systemic response of the traumatized patient: an overview. Vet Clin North Am Small Anim Pract 10: 523-32, 1980.

30. Bune, A. J., M. P. Brand, S. J. Heales, J. K. Shergill, R. Cammack, and H. T. Cook. Inhibition of tetrahydrobiopterin synthesis reduces in vivo nitric oxide production in experimental endotoxic shock. Biochem Biophys Res Commun 220: 139, 1996

31. Calignano, A., B. J. R. Whittle, M. DiRosa, and S. Moncada. Involvement of endogenous nitric oxide in the regulation of rat intestinal motility in vivo. Eur J Pharmacol 229: 273-276, 1992.

32. Castillo, L., A. Ajami, S. Branch, T. E. Chapman, Y. M. Yu, J. F. Burke, and V. R. Young. Plasma arginine kinetics in adult man: response to an arginine-free diet. Metabolism 43: 114-122, 1994.

33. Castillo, L., L. Beaumier, A. M. Ajami, and V. R. Young. Whole body nitric oxide synthesis in healthy men determined from [15N]-arginine-to[15N]citrulline labeling. Proc Natl Acad Sci USA 93: 11460-11465, 1996.

34. Castillo, L., T. E. Chapman, M. Sanchez, Y. M. Yu, J. F. Burke, A. M. Ajami, J. Vogt, and V. R. Young. Plasma arginine and citrulline kinetics in adults given adequate and arginine-free diets. Proc Natl Acad Sci USA 90: 7749-7753, 1993.

35. Cerra, F. B. Hypermetabolism-organ failure syndrome: a metabolic response to injury. Crit Care Clin 5: 289-302, 1989.

36. Chen, K., M. Inoue, and A. Okada. Expression of inducible nitric oxide synthase mRNA in rat digestive tissues after endotoxin and its role in intestinal mucosal injury. Biochem Biophys Res Comm 224: 703-708, 1996. 
37. Chiolero, R., J. P. Revelly, and L. Tappy. Energy metabolism in sepsis and injury. Nutrition 13: 45S-51S, 1997.

38. Chyun, J. H., and P. Griminger. Improvement of nitrogen retention by arginine and glycine supplementation and its relation to collagen synthesis in traumatized mature and aged rats. $J$ Nutr 114: 1697-704, 1984.

39. Cochard, A., R. Guilhermet, and M. Bonneau. Plasma growth hormone (GH), insulin and amino acid responses to arginine with or without aspartic acid in pigs. Effect of the dose. Reprod Nutr Dev 38: 331-43., 1998.

40. Colombo, J. P., U. Pfister, and H. Cervantes. The regulation of $\mathrm{N}$-acetylglutamate synthetase in rat liver by protein intake. Biochem Biophys Res Commun 172: 1239-45, 1990.

41. Cook, H. T., A. J. Bune, A. S. Jansen, G. M Taylor, R. K. Loi, and V. Cattell. Cellular localisation of inducible nitric oxide synthase in experimental endotoxic shock in the rat. $\mathrm{Clin} \mathrm{S \textrm {Ci }}$ 87: 179-186, 1994.

42. Cooney, R. N., S. R. Kimball, and T. C. Vary Regulation of skeletal muscle protein turnover during sepsis: mechanisms and mediators. Shock 7: 1-16, 1997.

43. Cori, C. F. The glucose-lactic acid cycle and gluconeogenesis. Curr Top Cell Regul 18: 377 $87,1981$.

44. Crawford, R. M., D. A. Leiby, S. J. Green, C. A. Nacy, A. H. Fortier, and M. S. Meltzer. Macrophage activation: a riddle of immunological resistance. Immunol Ser 60: 29-46, 1994.

45. Cui, X. L., M. Iwasa, Y. Iwasa, Y. Ohmori, A. Yamamoto, H. Maeda, M. Kume, S. Ogoshi, A. Yokoyama, T. Sugawara, and T. Funada. Effects of dietary arginine supplementation on protein turnover and tissue protein synthesis in scaldburn rats. Nutrition 15: 563-9, 1999.

46. Cullen, J. J., D. K. Caropreso, K. S. Ephgrave, L. L. Hemann, and M. M. Hinkhouse. The effect of endotoxin on canine motility and transit. $J$ Surg Res 67: 54-57, 1997

47. Cullen, J. J., D. Mercer, M. Hinkhouse, K. S. Ephgrave, and J. L. Conklin. Effects of endotoxin on regulation of intestinal smooth muscle nitric oxide synthase and intestinal transit. Surgery 125: 339-44., 1999.

48. Curran, R. D., F. K. Ferrari, P. H. Kispert, J. Stadler, D. J. Stuehr, R. L. Simmons, and T. R. Billiar. Nitric oxide and nitric oxide-generating compounds inhibit hepatocyte protein synthesis. Faseb J 5: 2085-92, 1991.

49. Cynober, L. Can arginine and ornithine support gut functions? Gut 35: S42-5., 1994.

50. Cynober, L. Ornithine alpha-ketoglutarate in nutritional support. Nutrition 7: 313-22., 1991.
51. Cynober, L., C. Coudray-Lucas, J. P. de Bandt, J. Guechot, C. Aussel, M. Salvucci, and J. Giboudeau. Action of ornithine alphaketoglutarate, ornithine hydrochloride, and calcium alpha-ketoglutarate on plasma amino acid and hormonal patterns in healthy subjects. $J$ Am Coll Nutr 9: 2-12., 1990.

52. Cynober, L., J. Le 'Boucher, and M. P. Vasson. Arginine metabolism in mammals. $J$ Nutr Biochem 6: 402-412, 1995.

53. Dahn, M. S., and P. Lange. Hormonal changes and their influence on metabolism and nutrition in the critically ill. Intensive Care Med 8: 209-13, 1982.

54. Dahn, M. S., R. A. Mitchell, M. P. Lange, S. Smith, and L. A. Jacobs. Hepatic metabolic response to injury and sepsis. Surgery 117: 521 $530,1995$.

55. Daly, J. M., J. Reynolds, A. Thom, L. Kinsley, M. Dietrick-Gallagher, J. Shou, and B. Ruggieri. Immune and metabolic effects of arginine in the surgical patient. Ann Surg 208: 512-23, 1988.

56. De Bandt, J. P., and L. A. Cynober. Amino acids with anabolic properties. Curr Opin Clin Nutr Metab Care 1: 263-72, 1998.

57. De Jonge, W. J., M. A. Dingemanse, P. A J. De Boer, W. H. Lamers, and A. F. M. Moorman. Arginine-metabolizing enzymes in the developing rat small intestine. Ped Res 43: 442-451, 1998.

58. Deutz, N. E. P., C. H. C. Dejong, G. Athanasas, and P. B. Soeters. Partial enterectomy in the rat does not diminish muscle glutamine production. Metabolism 41: 1343-1350, 1992.

59. Deves, R., and C. A. Boyd. Transporters for cationic amino acids in animal cells: discovery, structure, and function. Physiol Rev 78: 487-545, 1998.

60. Dhanakoti, S. N., J. T. Brosnan, G. R. Herzberg, and $M$. E. Brosnan. Renal arginine synthesis: studies in vitro and in vivo. Am J Physiol 259: E437-E442, 1990.

61. Doutheil, J., S. Althausen, M. Treiman, and W. Paschen. Effect of nitric oxide on endoplasmic reticulum calcium homeostasis, protein synthesis and energy metabolism [in Process Citation]. Cell Calcium 27: 107-15, 2000.

62. Dupre, J., J. D. Curtis, R. W. Waddell, and J. C. Beck. Alimentary factors in the endocrine response to administration of arginine in man. Lancet 2: 28-9, 1968.

63. Durante, W., L. Liao, I. Iftikhar, W. E. O'Brien, and A. I. Schafer. Differential regulation of Larginine transport and nitric oxide production by vascular smooth muscle and endothelium. Circ Res 78: 1075-82., 1996.

64. Edwards, A. V., M. A. Ghatei, and S. R. Bloom. Nitric oxide-related pancreatic endocrine responses to hyperglycaemia in the conscious calf. Experientia 50: 725-6, 1994. 
65. Evoy, D., M. D. Lieberman, T. J. Fahey, 3rd, and J. M. Daly. Immunonutrition: the role of arginine. Nutrition 14: 611-7, 1998.

66. Ewing, J. F., D. R. Janero, T. A. Grinnell, J. D. Schroeder, and D. S. Garvey. Reactivity of nitrogen monoxide species with $\mathrm{NADH}$ : implications for nitric oxide-dependent posttranslational protein modification. Arch Biochem Biophys 343: 131-9, 1997.

67. Felig, P. The glucose-alanine cycle. Metabolism 22: 179-207, 1973.

68. Fischer, J. E., and P. O. Hasselgren. Cytokines and glucocorticoids in the regulation of the "hepato-skeletal muscle axis" in sepsis. Am $J$ Surg 161: 266-71, 1991.

69. Floyd, J. C., Jr., S. S. Fajans, J. W. Conn, R. F. Knopf, and J. Rull. Stimulation of insulin secretion by amino acids. J Clin Invest 45: 1487 $502,1966$.

70. Flynn. N. E., and G. Wu. An important role for endogenous synthesis of arginine in maintaining arginine homeostasis in neonatal pigs. Am J Physiol 271: R1149-R1155, 1996.

71. Freund, H., S. Atamian, J. Holroyde, and J. E. Fischer. Plasma amino acids as predictors of the severity and outcome of sepsis. Ann Surg 190: 571-6, 1979.

72. Friedman, G., E. Silva, and J. L. Vincent. Has the mortality of septic shock changed with time [see comments]. Crit Care Med 26: 2078-86, 1998.

73. Gaginella, T. S., J. F. Kachur, H. Tamai, and A. Keshavarzian. Reactive oxygen and nitrogen metabolites as mediators of secretory diarrhea. Gastroenterology 109: 2019-28, 1995.

74. Galea, E., S. Regunathan, V. Eliopoulos, D. L. Feinstein, and D. J. Reis. Inhibition of mammalian nitric oxide synthases by agmatine, an endogenous polyamine formed by decarboxylation of arginine. Biochem J 316: 247 . 9, 1996.

75. Garthwaite, J., E. Southam, C. L. Boulton, E. B. Nielsen, K. Schmidt, and B. Mayer. Potent and selective inhibition of nitric oxide-sensitive guanylyl cyclase by $1 \mathrm{H}-[1,2,4]$ oxadiazolo $[4,3-$ a]quinoxalin-1-one. Mol Pharmacol 48: 184-8, 1995.

76. Gaston, B. Nitric oxide and thiol groups. Biochim Biophys Acta 1411: 323-33, 1999.

77. Gerber, N. C., C. R. Nishida, and P. R. Ortiz de Montellano. Characterization of human liver inducible nitric oxide synthase expressed in Escherichia coli. Arch Biochem Biophys 343: 249-53, 1997.

78. Gill, D. J., B. C. Low, and M. R. Grigor. Interleukin-1 beta and tumor necrosis factoralpha stimulate the cat-2 gene of the L-arginine transporter in cultured vascular smooth muscle cells. J Biol Chem 271: 11280-3, 1996.
79. Giugliano, D., R. Marfella, G. Verrazzo, R. Acampora, L. Coppola, D. Cozzolino, and F. D'Onofrio. The vascular effects of L-Arginine in humans. The role of endogenous insulin. $J$ Clin Invest 99: 433-8, 1997.

80. Granger, D. L., J. B. Hibbs, Jr., J. R. Perfect, and D. T. Durack. Metabolic fate of L-arginine in relation to microbiostatic capability of murine macrophages. J Clin Invest 85: 264-73, 1990.

81. Green, L. C., K. Ruiz de Luzuriaga, D. A. Wagner, W. Rand, N. Istfan, V. R. Young, and S. R. Tannenbaum. Nitrate biosynthesis in man. Proc Natl Acad Sci U S A 78: 7764-8, 1981.

82. Green, S. J., and C. A. Nacy. Antimicrobial and immunopathologic effects of cytokine-induced nitric oxide synthesis. Curr Opin Infect Dis 6: 384-396, 1993.

83. Hammarqvist, F., B. Ejesson, and J. Wernerman. Stress hormones initiate prolonged changes in the muscle amino acid pattern. Clin Physiol 21: 44-50., 2001.

84. Hattori, Y., E. B. Campbell, and S. S. Gross. Argininosuccinate synthetase mRNA and activity are induced by immunostimulants in vascular smooth muscle. Role in the regeneration of arginine for nitric oxide synthesis. $J$ Biol Chem 269: 9405-9408, 1994

85. Hattori, Y., K. Kasai, and S. S. Gross. Cationic amino acid transporter gene expression in cultured vascular smooth muscle cells and in rats. Am J Physiol 276: H2020-8, 1999.

86. Hayashi, T., M. F. Hirshman, N. Fujii, S. A. Habinowski, L. A. Witters, and L. J. Goodyear. Metabolic stress and altered glucose transport: activation of AMP-activated protein kinase as a unifying coupling mechanism. Diabetes 49: 527 31., 2000.

87. Hecker, M., W. C. Sessa, H. J. Harris, E. E. Anggard, and J. R. Vane. The metabolism of Larginine and its significance for the biosynthesis of endothelium-derived relaxing factor: cultured endothelial cells recycle $L$-citrulline to $\mathrm{L}$-arginine. Proc Natl Acad Sci U S A 87: 8612-6, 1990.

88. Henderson, J. L., R. Statman, J. N. Cunningham, W. Cheng, P. Damiani, A. Siconolfi, and J. H. Horovitz. The effects of nitric oxide inhibition on regional hemodynamics during hyperdynamic endotoxemia. Arch Surg 129: 1271-1275, 1994.

89. Hibbs, J. B., Jr., C. Westenfelder, R. Taintor, Z. Vavrin, C. Kablitz, R. L. Baranowski, J. H. Ward, R. L. Menlove, M. P. McMurry, J. P. Kushner, and et al. Evidence for cytokine-inducible nitric oxide synthesis from L-arginine in patients receiving interleukin-2 therapy [published erratum in J Clin Invest 1992 Jul;90(1):295]. J Clin Invest 89: 867-77, 1992.

90. Hinshaw, L. B., L. A. Solomon, D. D. Holmes, and L. J. Greenfield. Comparison of canine responses to Escherichia coli organisms and endotoxin. Surg Gynecol Obstet 127: 981-8, 1968. 
91. Hom, G. J., S. K. Grant, G. Wolfe, T. J. Bach, D. E. Macintyre, and N. I. Hutchinson. Lipopolysaccharide-induced hypotension and vascular hyporeactivity in the rat: tissue analysis of nitric oxide synthase mRNA and protein expression in the presence and absence of dexamethasone, $\mathrm{Ng}$-monomethyl-L-arginine or indomethacin. J Pharmacol Exp Ther 272: 452$459,1995$.

92. Hoogenraad, N., N. Totino, H. Elmer, C. Wraight, P. Alewood, and R. B. Johns. Inhibition of intestinal citrulline synthesis causes severe growth retardation in rats. Am J Physiol 249: G792-9, 1985.

93. Hosomi, M., N. H. Stace, F. Lirussi, S. M. Smith, G. M. Murphy, and R. H. Dowling. Role of polyamines in intestinal adaptation in the rat. Eur $J$ Clin Invest 17: 375-85, 1987.

94. Hrabak, A., T. Bajor, and A. Temesi. Comparison of substrate and inhibitor specificity of arginase and nitric oxide (NO) synthase for arginine analogues and related compounds in murine and rat macrophages. Biochem Biophys Res Commun 198: 206-12, 1994.

95. Ignarro, L. J., H. Lippton, J. C. Edwards, W. H. Baricos, A. L. Hyman, P. J. Kadowitz, and C. A. Gruetter. Mechanism of vascular smooth muscle relaxation by organic nitrates, nitrites, nitroprusside and nitric oxide: evidence for the involvement of S-nitrosothiols as active intermediates. J Pharmacol Exp Ther 218: 739749, 1981.

96. Imms, F. J., D. R. London, and R. L. Neame. The secretion of catecholamines from the adrenal gland following arginine infusion in the rat. J Physiol (Lond) 200: 55P-6P, 1969.

97. Inoue, Y., B. P. Bode, D. J. Beck, A. P. Li, K. I. Bland, and W. W. Souba. Arginine transport in human liver. Characterization and effects of nitric oxide synthase inhibitors. Ann Surg 218: 350363, 1993.

98. Isidori, A., A. Lo Monaco, and M. Cappa. A study of growth hormone release in man after oral administration of amino acids. Curr Med Res Opin 7: 475-81, 1981.

99. Iwata, F., T. Joh, T. Kawai, and M. Itoh. Role of EDRF in splanchnic blood flow of normal and chronic portal hypertensive rats. Am $J$ Physiol 263: G149-54, 1992.

100. Jenkinson, C. P., W. W. Grody, and S. D. Cederbaum. Comparative properties of arginases. Comp Biochem Physiol B Biochem Mol Biol 114: 107-32, 1996.

101. Jeremy, R. W., H. McCarron, and D. Sullivan. Effects of dietary L-arginine on atherosclerosis and endothelium- dependent vasodilatation in the hypercholesterolemic rabbit. Response according to treatment duration, anatomic site, and sex. Circulation 94: 498-506, 1996.
102. Kakuda, D. K., M. J. Sweet, C. L. Mac Leod, D. A. Hume, and D. Markovich. CAT2-mediated Larginine transport and nitric oxide production in activated macrophages. Biochem J 340: 549-53, 1999.

103. Kang, Y. H., C. H. Lee, R. L. Monroy, R. S. Dwivedi, C. Odeyale, and H. H. Newball. Uptake, distribution and fate of bacterial lipopolysaccharides in monocytes and macrophages: an ultrastructural and functional correlation. Electron Microsc Rev 5: $381-419$, 1992.

104. Kelly, E., S. M. Morris, Jr., and T. R. Billiar. Nitric oxide, sepsis, and arginine metabolism. JPEN $J$ Parenter Enteral Nutr 19: 234-8, 1995.

105. Kieft, H., A. I. Hoepelman, W. Zhou, M. Rozenberg-Arska, A. Struyvenberg, and J. Verhoef. The sepsis syndrome in a Dutch university hospital. Clinical observations. Arch Intern Med 153: 2241-7., 1993.

106. Kim, J. W., E. I. Closs, L. M. Albritton, and J. M. Cunningham. Transport of cationic amino acids by the mouse ecotropic retrovirus receptor [see comments]. Nature 352: 725-8, 1991.

107. Kirkeboen, K. A., and O. A. Strand. The role of nitric oxide in sepsis-an overview. Acta Anaesthesiol Scand 43: 275-88, 1999.

108. Kitamura, T., J. Yasuda, and A. Hashimoto. Acute insulin response to intravenous arginine in nonobese healthy cats. J Vet Intern Med 13: 549-56, 1999.

109. Knopf, R. F., J. W. Conn, and S. S. Fajans. Plasma growth hormone response to intravenous administration of amino acids. J Clin Endocrinol 25: 1140-1144, 1965.

110. Kobzik, L., M. B. Reid, D. S. Bredt, and J. S. Stamler. Nitric oxide in skeletal muscle. Nature 372: 546-8, 1994.

111. Kreimeier, U., U. B. Brueckner, S. Gerspach, K. Veitinger, and K. Messmer. A porcine model of hyperdynamic endotoxemia: pattern of respiratory, macrocirculatory, and regional blood flow changes. J Invest Surg 6: 143-156, 1993.

112. Kubes, P., M. Suzuki, and D. N. Granger. Nitric oxide: an endogenous modulator of leukocyte adhesion. Proc Natl Acad Sci U S A 88: 4651-5. 1991.

113. Kudsk, K. A., J. M. Stone, and G. F. Sheldon. Nutrition in trauma and burns. Surg Clin North Am 62: 183-92, 1982

114. Lavenson, S. M., G. Rettura, and A. Barbul Citrulline replaces arginine as a dietary essential in rats: ornithine does not. Fed Proc 39: 726, 1980.

115. Lawler, D. A. Hormonal response in sepsis. Crit Care Nurs Clin North Am 6: 265-74, 1994. 
116. Le Bricon, T., C. Coudray-Lucas, N. Lioret, S. K. Lim, F. Plassart, L. Schlegel, J. P. De Bandt, R. Saizy, J. Giboudeau, and L. Cynober. Ornithine alpha-ketoglutarate metabolism after enteral administration in burn patients: bolus compared with continuous infusion. Am J Clin Nutr 65: 5128., 1997.

117. Lorente, J. A., M. A. Delgado, C. Tejedor, E. Mon, M. Hervas, T. Pascual, P. FernandezSegoviano, G. Rieppi, A. Soler, D. Ayuso, and A. Esteban. Modulation of systemic hemodynamics by exogenous L-arginine in normal and bacteremic sheep. Crit Care Med 27: 2474-9. 1999.

118. MacAllister, R. J., A. L. Calver, J. Collier, C. M. Edwards, B. Herreros, S. S. Nussey, and $P$. Vallance. Vascular and hormonal responses to arginine: provision of substrate for nitric oxide or non-specific effect? Clin Sci (Colch) 89: 183-90, 1995.

119. Malandro, M. S., and M. S. Kilberg. Molecular biology of mammalian amino acid transporters. Annu Rev Biochem 65: 305-36, 1996.

120. Manteuffel-Cymborowska, M., W. Chmurzynska, M. Peska, and B. Grzelakowska-Sztabert. Arginine and ornithine metabolizing enzymes in testosterone-induced hypertrophic mouse kidney. Int J Biochem Cell Biol 27: 287-95, 1995.

121. Marescau, B., D. R. Deshmukh, M. Kockx, I. Possemiers, I. A. Qureshi, P. Wiechert, and P. P. De Deyn. Guanidino compounds in serum, urine, liver, kidney, and brain of man and some ureotelic animals. Metabolism 41: 526-32, 1992.

122. Marsh, C. B., and M. D. Wewers. The pathogenesis of sepsis. Factors that modulate the response to gram- negative bacterial infection. Clin Chest Med 17: 183-97, 1996.

123. Martinez, M. J., and J. Giraldez. Plasma aminogram in critical patients. Nutr Hosp 8: 79$93,1993$.

124. Matthews, D. E., and A. Battezzati. Regulation of protein metabolism during stress. Curr Opin Gen Surg: 72-7, 1993.

125. McGuire, D. M., M. D. Gross, R. P. Elde, and J. F. van Pilsum. Localization of L-arginine-glycine amidinotransferase protein in rat tissues by immunofluorescence microscopy. J Histochem Cytochem 34: 429-35, 1986.

126. Meijer, A. J., W. H. Lamers, and R. A. F. M. Chamuleau. Nitrogen metabolism and ornithine cycle function. Physiol Rev 70: 701-748, 1990.

127. Merimee, T. J., D. A. Lillicrap, and D. Rabinowitz. Effect of arginine on serum-levels of human growth-hormone. Lancet 2: 668-70, 1965.

128. Meszaros, K., C. H. Lang, G. J. Bagby, and J. J. Spitzer. In vivo glucose utilization by individual tissues during nonlethal hypermetabolic sepsis. Faseb J 2: 3083-6, 1988.
129. Millward, D. J., P. J. Garlick, W. P. T. James, P. M. Sender, and J. C. Waterlow. Protein turnover. in: Protein metabolism and nutrition, edited by $D$. J. A. Cole, K. N. Boorman, P. J. Buttery, D. Lewis, R. J. Neale and $H$. Swan. London: Butterworths, 1976, p. 49-69.

130. Mitsui, Y., and S. Furuyama. Characterization of nitric oxide synthase in the rat parotid gland. Arch Oral Biol 45: 531-6, 2000.

131. Moncada, S., R. M. J. Palmer, and E. A. Higgs. Nitric oxide: physiology, pathophysiology and pharmacology. Pharmacol Rev 13: 109-142, 1991.

132. Morris, S. M. j. Regulation of enzymes of urea and arginine synthesis. Annu Rev Nutr 12: 81 $101,1992$.

133. Morris, S. M. j., and T. R. Billiar. New insights into the regulation of inducible nitric oxide synthesis. Am J Physiol 266: E829-E839, 1994.

134. Murphy, C., and P. Newsholme. Importance of glutamine metabolism in murine macrophages and human monocytes to L-arginine biosynthesis and rates of nitrite or urea production. Clin Sci (Colch) 95: 397-407, 1998.

135. Muscara, M. N., and J. L. Wallace. Nitric Oxide. $V$. therapeutic potential of nitric oxide donors and inhibitors. Am J Physiol 276: G1313-6, 1999

136. Nagasaki, A., T. Gotoh, M. Takeya, Y. Yu, M. Takiguchi, H. Matsuzaki, K. Takatsuki, and $M$. Mori. Coinduction of nitric oxide synthase, argininosuccinate synthetase, and arginiosuccinate lyase in lipopolysaccharidetreated rats. J Biol Chem 271: 2658-2662, 1996.

137. Natanson, C. W. D. Hoffman, A. F. Suffredini, P. Q. Eichacker, and R. L. Danner. Selected treatment strategies for septic shock based on proposed mechanisms of pathogenesis. Ann Intern Med 120: 771-83, 1994.

138. Nathan, C. Nitric oxide as a secretory product of mammalian cells. Faseb $J$ 6: 3051-64, 1992.

139. Nathan, C., and Q. W. Xie. Nitric oxide synthases: roles, tolls, and controls. Cell 78: 915-8, 1994.

140. Nawabi, M. D., K. P. Block, M. C. Chakrabarti, and M. G. Buse. Administration of endotoxin, tumor necrosis factor, or interleukin 1 to rats activates skeletal muscle branched-chain alphaketo acid dehydrogenase. J Clin Invest 85: 256 63,1990

141. Newsholme, E. A., and A. R. Leech. Biochemistry for the medical sciences. New York: John Wiley \& Sons, 1983. p. 1-952.

142. Nicholson, B., T. Sawamura, T. Masaki, and C. L. MacLeod. Increased Cat3-mediated cationic amino acid transport functionally compensates in Cat1 knockout cell lines. $J$ Biol Chem 273: 14663-6., 1998. 
143. Nussler, A. K., and T. R. Billiar. Inflammation, immunoregulation, and inducible nitric oxide synthase. J Leukoc Biol 54: 171-8, 1993.

144. Nussler, A. K., T. R. Billiar, Z. Z. Liu, and S. M. j. Morris. Coinduction of nitric oxide synthase and argininosuccinate synthetase in a murine macrophage cell line. Implications for regulation of nitric oxide production. J Biol Chem 269: 1257-1261, 1994.

145. Ohshima, H., M. Friesen, I. Brouet, and $H$. Bartsch. Nitrotyrosine as a new marker for endogenous nitrosation and nitration of proteins. Fd Chem Toxic 28: 647-652, 1996.

146. Oka, T., K. Ohwada, M. Nagao, K. Kitazato, and $Y$. Kishino. Arginine-enriched solution induces a marked increase in muscle glutamine concentration and enhances muscle protein synthesis in tumor- bearing rats. JPEN $J$ Parenter Enteral Nutr 18: 491-6, 1994.

147. O'Sullivan, D., J. T. Brosnan, and M. E. Brosnan. Hepatic zonation of the catabolism of arginine and orinthine in the perfused rat liver. Biochem $J$ 330: 627-632, 1998.

148. Pacitti, A. J., E. M. Copeland, and W. W. Souba. Stimulation of hepatocyte system y+-mediated Larginine transport by an inflammatory agent. Surgery 112: 403-411, 1992.

149. Palmer, J. P., R. M. Walter, and J. W. Ensinck. Arginine-stimulated acute phase of insulin and glucagon secretion. 1. in normal man. Diabetes 24: 735-40, 1975.

150. Palmer, R., D. Ashton, and S. Moncada. Vascular endothelial cells syntesize nitric oxide from L-arginine. Nature 333: 664-666, 1988.

151. Pardridge, W. M., L. Duducgian-Vartavarian, D. Casanello-Ertl, M. R. Jones, and J. D. Kopple. Arginine metabolism and urea synthesis in cultured rat skeletal muscle cells. Am J Physiol 242: E87-92, 1982.

152. Park, J. H., S. H. Chang, K. M. Lee, and S. H. Shin. Protective effect of nitric oxide in an endotoxin-induced septic shock. Am J Surg 171: 340-5, 1996.

153. Pastor, C. M., and T. R. Billiar. Nitric oxide causes hyporeactivity to phenylephrine in isolated perfused livers from endotoxin-treated rats. Am J Physiol 268: G177-G182, 1995.

154. Pegg, A. E. Recent advances in the biochemistry of polyamines in eukaryotes. Biochem $J 234$ : 249-62, 1986.

155. Peretz, D. I., H. M. Scott, J. Duff, J. B. Dossetor, L. D. MacLean, and M. McGregor. The significance of lacticacidemia in the shock syndrome. Ann N Y Acad Sci 119: 1133-41, 1965.

156. Pinnell, S. R. Regulation of collagen synthesis. $J$ Invest Dermatol 79 Suppl 1: 73s-76s, 1982.
157. Prins, H. A., A. P. Houdijk, M. J. Wiezer, T. Teerlink, A. A. van Lambalgen, L. G. Thijs, and P. A. van Leeuwen. Reduced arginine plasma levels are the drive for arginine production by the kidney in the rat. Shock 11: 199-204, 1999.

158. Pui, Y. M., and H. Fisher. Factorial supplementation with arginine and glycine on nitrogen retention and body weight gain in the traumatized rat. J Nutr 109: 240-6, 1979.

159. Rabier, D., C. Narcy, J. Bardet, P. Parvy, J. M. Saudubray, and $P$. Kamoun. Arginine remains an essential amino acid after liver transplantation in urea cycle enzyme deficiencies. $J$ Inherit Metab Dis 14: 277-80, 1991.

160. Rakoff, J. S., T. M. Siler, Y. N. Sinha, and S. S. Yen. Prolactin and growth hormone release in response to sequential stimulation by arginine and synthetic TRF. J Clin Endocrinol Metab 37: $641-4,1973$.

161. Rebuffat, P., C. Macchi, L. K. Malendowicz, and G. G. Nussdorfer. Comparison of the signaling mechanisms involved in the ETB receptormediated secretagogue action of endothelin- 1 on dispersed zona glomerulosa cells and capsulezona glomerulosa preparations of the rat adrenal gland. Int J Mol Med 5: 43-47, 2000.

162. Rennie, M. J. Muscle protein turnover and the wasting due to injury and disease. $\mathrm{Br}$ Med Bull 41: 257-64, 1985.

163. Reyes, A. A., I. E. Karl, and S. Klahr. Role of arginine in health and in renal disease. Am J Physiol 267: F331-46, 1994.

164. Reynolds, J. V., J. M. Daly, J. Shou, R. Sigal, M. M. Ziegler, and A. Naji. Immunologic effects of arginine supplementation in tumor-bearing and non-tumor-bearing hosts. Ann Surg 211: 202210, 1990.

165. Rodriguez-Membrilla, A., V. Martinez, $M$. Jiminez, E. Goñalons, and P. Vegara. Is nitric oxide the final mediator regulating the migrating myoelectric complex cycle? Gastrointest Liver Physiol 268: G207-G214, 1995.

166. Salimuddin, A. Nagasaki, T. Gotoh, H. Isobe, and $M$. Mori. Regulation of the genes for arginase isoforms and related enzymes in mouse macrophages by lipopolysaccharide. Am J Physiol 277: E110-7, 1999.

167. Salter, M., R. G. Knowles, and S. Moncada. Widespread tissue distribution, species distribution and changes in activity of $\mathrm{Ca2}+$ deperident and $\mathrm{Ca} 2+-$ independent nitric oxide synthases. FEBS Lett 291: 145-149, 1991.

168. Sarna, S. K., M. F. Otterson, R. P. Ryan, and V. E. Cowles. Nitric oxide regulates migrating motor complex cycling and its postprandial disruption. Am J Physiol 265: G759-G766, 1993.

169. Sato, H., M. Fujiwara, and S. Bannai. Effect of lipopolysaccharide on transport and metabolism of arginine in mouse peritoneal macrophages. $J$ Leukoc Biol 52: 161-164, 1992. 
170. Satriano, J., C. J. Kelly, and R. C. Blantz. An emerging role for agmatine. Kidney int 56: 1252 3, 1999.

171. Satriano, J., S. Matsufuji, Y. Murakami, M. J. Lortie, D. Schwartz, C. J. Kelly, S. Hayashi, and R. C. Blantz. Agmatine suppresses proliferation by frameshift induction of antizyme and attenuation of cellular polyamine levels. $J$ Biol Chem 273: 15313-6, 1998.

172. Schott, C. A., G. A. Gray, and J. C. Stoclet. Dependence of endotoxin-induced vascular hyporeactivity on extracellular L-arginine. $\mathrm{Br} J$ Pharmacol 108: 38-43., 1993.

173. Shibazaki, T., M. Fujiwara, H. Sato, K. Fujiwara, K. Abe, and S. Bannai. Relevance of the arginine transport activity to the nitric oxide synthesis in mouse peritoneal macrophages stimulated with bacterial lipopolysaccharide. Biochim Biophys Acta 1311: 150-154, 1996.

174. Siegel, J. H., T. C. Vary, A. Rivkind, R. Bilik, B. Coleman, B. E. Tall, and J. G. Morris. Abnormal metabolic control in the septic multiple organ failure syndrome: pharmacotherapy for altered fuel control mechanisms. Prog Clin Biol Res 308: 535-43, 1989.

175. Skeie, B., V. Kvetan, K. M. Gil, M. M. Rothkopf, E. A. Newsholme, and J. Askanazi. Branch-chain amino acids: their metabolism and clinical utility. Crit Care Medicine 18: 549-571, 1990.

176. Soeters, P. B., and N. E. P. Deutz. Interorgan exchange of amino acids after trauma. Organ metabolism and nutrition: Ideas for future critical care

\section{Ed: Kinney JM, Tucker HN}

Raven Press Ltd, New York, USA:, 1994.

177. Sonoda, M., J. Kobayashi, M. Takezawa, T. Miyazaki, T. Nakajima, H. Shimomura, K. Koike, A. Satomi, H. Ogino, R. Omoto, and T. Komoda. An assay method for nitric oxide-related compounds in whole blood. Anal Biochem 247: 417-27, 1997.

178. Spitzer, J. J., G. J. Bagby, K. Meszaros, and C. $H$. Lang. Altered control of carbohydrate metabolism in endotoxemia. Prog Clin Biol Res 286: 145-65, 1989.

179. Sprangers, F., H. P. Sauerwein, J. A. Romijn, G. M. van Woerkom, and A. J. Meijer. Nitric oxide inhibits glycogen synthesis in isolated rat hepatocytes. Biochem J 330: 1045-9, 1998.

180. Stadler, J., J. Barton, H. Beil-Moelier, S. Diekmann, C. Hierholzer, W. Erhard, and C. D. Heidecke. Hepatocyte nitric oxide biosynthesis inhibits glucose output and competes with urea synthesis for L-arginine. Am J Physiol 268: G183-G188, 1995.

181. Stark, M. E., A. J. Bauer, and J. H. Szurszewski. Effect of nitric oxide on circular muscle of the canine small intestine. J Physiol (Lond) 444: 743-61, 1991.
182. Stoll, B., J. Henry, P. J. Reeds, H. Yu, F. Jahoor, and D. G. Burrin. Catabolism dominates the firstpass intestinal metabolism of dietary essential amino acids in milk protein-fed piglets. $J$ Nutr 128: 606-14, 1998

183. Tabor, C. W., and H. Tabor. Polyamines. Annu Rev Biochem 53: 749-90, 1984.

184. Tang, G. J. [Similarity and synergy of trauma and sepsis: role of tumor necrosis factor-alpha and interleukin-6 (see comments)]. Acta Anaesthesiol $\sin 34: 141-9,1996$.

185. Thompson, J. S. The intestinal response to critical illness. Am J Gastroenterol 90: 190-200, 1995.

186. Tizianello, A., G. De Ferrari, G. Garibotto, and C. Robaudo. Amino acid metabolism and the liver in renal failure. Am J Clin Nutr 33: 1354-62, 1980.

187. Utsumi, M., H. Makimura, K. Ishihara, S. Morita, and $S$. Baba. Determination of immunoreactive somatostatin in rat plasma and responses to arginine, glucose and glucagon infusion. Diabetologia 17: 319-23, 1979.

188. Van Broeckhoven, C. L., K. M. Adriaenssens, B. Marescau, J. Pintens, and H. G. Terheggen. Measurement of arginine transport in human erythrocytes using their intrinsic arginase activity: implications for the treatment of familial hyperargininemia. Clin Chim Acta 126: 209-16, 1982.

189. Vary, T. C. Inter-organ protein and carbohydrate metabolic relationships during sepsis: necessary evils or uncanny coincidences? Curr Opin Clin Nutr Metab Care 2: 235-42, 1999.

190. Venturi, C. M., R. M. J. Palmer, and S. Moncada. Enhancement of cellular proliferation follows inhibition of nitric oxide formation. In: The biology of nitric oxide. 1. Physiological and clinical aspects, edited by S. Moncada, M. A. Marletta, J. B. j. Hibbs and E. A. Higgs. London: Portland Press, 1992, p. 208-210.

191. Vincent, S. R., and B. T. Hope. Neurons that say NO. Trends Neurosci 15: 108-13, 1992.

192. Visek, W. J. Arginine and disease states. J Nutr 115: 532-41, 1985.

193. Visek, W. J. Arginine needs, physiological state and usual diets. A reevaluation. J Nutr 116: 36 $46,1986$.

194. Visek, W. J. An update of concepts of essential amino acids. Annu Rev Nutr 4: 137-55, 1984.

195. Vromen, A., M. S. Arkovitz, B. Zingarelli, A. L. Salzman, V. F. Garcia, and C. Szabo. Low-level expression and limited role for the inducible isoform of nitric oxide synthase in the vascular hyporeactivity and mortality associated with cecal ligation and puncture in the rat. Shock 6: 248-53, 1996. 
196. Wakabayashi, Y. The glutamate crossway. In: Amino acid metabolism and therapy in health and nutritional disease, edited by $L$. Cynober. FL USA: CRC Press, Boca raton, 1985, p. 89-98.

197. Wakabayashi, Y. Tissue-selective expression of enzymes of arginine synthesis. Curr Opin Clin Nutr Metab Care 1: 335-9., 1998.

198. Weimann, A., L. Bastian, W. E. Bischoff, M. Grotz, M. Hansel, J. Lotz, C. Trautwein, G. Tusch, H. J. Schlitt, and G. Regel. Influence of arginine, omega- 3 fatty acids and nucleotidesupplemented enteral support on systemic inflammatory response syndrome and multiple organ failure in patients after severe trauma. Nutrition 14: 165-72, 1998.

199. Weir, G. C., E. Samols, S. Loo, Y. C. Patel, and K. H. Gabbay. Somatostatin and pancreatic polypeptide secretion: effects of glucagon, insulin, and arginine. Diabetes 28: 35-40, 1979.

200. Welters, C. F., C. H. Dejong, N. E. Deutz, and E. Heineman. Effects of parenteral arginine supplementation on the intestinal adaptive response after massive small bowel resection in the Rat. J Surg Res 85: 259-66, 1999.

201. Wenzel, R. P. The mortality of hospital-acquired bloodstream infections: need for a new vital statistic? Int J Epidemiol 17: 225-7, 1988.

202. White, M. F. The transport of cationic amino acids across the plasma membrane of mammalian cells. Biochim Biophys Acta 822: 355-74, 1985.

203. Windmueller, H. G., and A. E. Spaeth. Source and fate of circulating citrulline. Am J Physiol 241: E473-E480, 1981.

204. Windsor, J. A., and G. L. Hill. Grip strength: a measure of the proportion of protein loss in surgical patients. Br J Surg 75: 880-2, 1988.

205. Wink, D. A., K. M. Miranda, M. G. Espey, R. M. Pluta, S. J. Hewett, C. Colton, M. Vitek, M. Feelisch, and M. B. Grisham. Mechanisms of the antioxidant effects of nitric oxide. Antioxid Redox Signal 3: 203-13., 2001.
206. Wirthlin, D. J., J. J. Cullen, S. T. Spates, J. L. Conklin, J. Murray, D. K. Caropreso, and K. S. Ephgrave. Gastrointestinal transit during endotoxemia: the role of nitric oxide. $J$ Surg Res 60: 307-311, 1996.

207. Wolff, D. J., and G. A. Datto. Identification and characterization of a calmodulin-dependent nitric oxide synthase from $\mathrm{GH} 3$ pituitary cells. Biochem J 285: 201-6, 1992.

208. Wu, G., P. K. Davis, N. E. Flynn, D. A. Knabe, and J. T. Davidson. Endogenous synthesis of arginine plays an important role in maintaining arginine homeostasis in postweaning pigs. J Nutr 127: 2342-2349, 1997

209. Wu, G., N. E. Flynn, S. P. Flynn, C. A. Jolly, and P. K. Davis. Dietary protein or arginine deficiency impairs constitutive and inducible nitric oxide synthesis by young rats. J Nutr 129: 1347-54, 1999.

210. Wu, G., D. A. Knabe, N. E. Flynn, W. Yan, and S. P. Flynn. Arginine degradation in developing porcine enterocytes. Am J Physiol 271: G913G919, 1996.

211. Wu, G., and S. M. Morris, Jr. Arginine metabolism: nitric oxide and beyond. Biochem $J$ 336: 1-17, 1998.

212. Wyss, M., and R. Kaddurah-Daouk. Creatine and creatinine metabolism. Physiol Rev 80: 1107$213,2000$.

213. Xie, L., Y. Hattori, N. Tume, and S. S. Gross. The preferred source of arginine for high-output nitric oxide synthesis in blood vessels. Semin Perinatol 24: 42-5., 2000.

214. Xie, Q. W., H. J. Cho, J. Calaycay, R. A. Mumford, K. M. Swiderek, T. D. Lee, A. Ding, T. Troso, and C. Nathan. Cloning and characterization of inducible nitric oxide synthase from mouse macrophages. Science 256: 225-8, 1992.

215. Yu, Y. M., J. F. Burke, R. G. Tompkins, R. Martin, V. R. Young, and M. E. Brosnan. Quantitative effects of interorgan relationships among arginine and citrulline metabolism. Am J Physiol 271: E437-E442, 1996. 


\title{
SEPSIS AND METABOLIC MEASUREMENT MODELS
}

\author{
Maaike J Bruins \\ Peter B Soeters \\ Nicolaas EP Deutz
}

Department of Surgery, Maastricht University, The Netherlands 


\section{Introduction}

In this section, the experimental model of sepsis that was used in this thesis will be defined in more detail. Moreover, an explanatory description of the different applied techniques will be given. The aspects of indicator dilution techniques to measure organ plasma flow and tracer and stable isotope methods to measure specific substrate kinetics in the whole body and across the organs are outlined.

\section{Model of sepsis}

\subsection{Clinical background}

Despite intensive care, sepsis is still a major cause of death throughout the world. Sepsis is associated with a very wide range of disorders, such as profound hemodynamic, nutritional derangements, organ failure and multiple metabolic alterations with increased energy substrate turnover, altered hormone pattern and severe changes in protein metabolism. Sepsis was defined as a generalized inflammatory response that is accompanied by acute organ dysfunction and is often associated with the presence of bacterial, fungal or virus infection (5). Findings of generalized inflammation include fever, tachycardia, tachypnea, low systemic vascular resistance, leucocytosis, thrombocytopenia, and altered metal status. Sepsis may involve circulatory decompensation (i.e., hypotension) related to sepsis-induced increases in vascular leak. When hypotension and hypovolemia result in compromised cardiac output and organ perfusion this can lead to irreversible "septic shock". However, in humans with compensated sepsis, cardiac output is typically elevated and systemic vascular resistance is usually abnormally low. Metabolic characteristics are increased levels of circulating stress hormones, net protein breakdown, increased gluconeogenesis, ureagenesis and ketogenesis.

\subsection{Existing models}

Bacterial endotoxins are often used in experimental models to study the mechanisms involved in inflammation and sepsis. Large doses of endotoxin lead to a hypodynamic profile leading to circulatory collapse (i.e. hypotension) and septic shock (5). However, in severely ill patients splanchnic hypoperfusion causes slow endotoxin release from the gut (9). This leakage of small amounts of endotoxin into the circulation elicits a hyperdynamic sepsis response. Therefore, very small doses of endotoxin are more representative for hypermetabolic human sepsis. However, most of the experimental models of sepsis are very severe and present a high degree of mortality. Many animal 
models of sepsis were developed aiming at the clinical relevance. Nevertheless, their hemodynamic features and aspects as metabolism and pulmonary function largely differ. The variability in the models depends on confounding factors as age, species and supportive therapy.

Four main approaches for producing sepsis in laboratory animals can be recognized:

(a) Induction of peritonitis using a defined bacterial inoculum or endotoxin, (b) cecal ligation and perforation (CLP), (c) induction of soft tissue abscesses in extremities and (d) intravascular infusion of live bacteria or endotoxin.

(a) Bacterial inoculum This model of sepsis involves quantification of bacteria and implantation of suspended bacteria or endotoxin intraperitoneally. Despite complete control over the dose of bacteria, high mortality occurs after onset of peritonitis.

(b) Cecal ligation and perforation

The model of cecal ligation and perforation (CLP) is a model of sepsis due to peritoneal contamination with mixed flora by cecal perforation. In this model that has extensively used in small laboratory animals, sepsis is easily induced by a surgical procedure without introduction of bacteria. However, in this model it is difficult to control the magnitude of the septic challenge. Prolonged survival has been described in a modification of the CLP model that uses inoculation of the peritoneal cavity with fecal material. However, standardization of this model is still very difficult for metabolic studies due to a large variability in the severity of disease; also high mortality ( $76 \%$ within 5 days) is observed in this model (10).

(c) Soft tissue abscesses This procedure involves injection of an infective agent (including bacteria and endotoxins) in tissue of the muscle, by intramuscular injection, creating a local site infection and a hyperdynamic circulation (13). This model suffers from considerable variability, strongly depending upon the endotoxin or strain of bacteria used.

(d) Intravenous infusion The intravascular administration of bolus or continuous infusion of bacteria or endotoxin is an other approach that is often used to imitate sepsis. High dose of endotoxin or bacteria by means of a bolus injection induces an overwhelming insult resulting in high mortality within a few hours (see for review: (1)). Also, hemodynamic perturbations elicited by bolus injections of large doses endotoxin are quite different from human compensated sepsis and generally have the characteristics of hypodynamic sepsis with low cardiac output and normal or elevated vascular resistance $(2,4,7,8)$. In view of the likelihood that in most patients with sepsis endotoxins are released into the circulation over an extended period, continuous intravenous administration of low-dose endotoxins or bacteria better resemble human sepsis (5). Low doses of endotoxin evoke a profile characteristic of the hyperdynamic 
response (4). Because sepsis involves circulatory decompensation (i.e., hypotension) related to increased vascular leak, adequate volume loading is often applied to secure replenishment of intravascular volume losses and, thus, to assure a hyperdynamic profile of sepsis.

Therefore, to reproduce a hyperdynamic profile in which hypovolemia-related cardiac output and organ perfusion is well preserved, animals should be administrated low amounts of endotoxin and high amounts of fluid.

\subsection{Choice of the model}

Animals Small animals are desirable for sepsis experiments because they are easy to acquire and maintain, particularly when large numbers of conditions are studied. Nevertheless, hemodynamic variables are easier to study in larger animals. Because of their greater circulating volume, these larger species are also useful when multiple blood samples are desired. Pigs are very similar to humans with respect to renal, cardiovascular and digestive anatomy and physiology $(3,12)$. Since pigs are docile animals and relatively inexpensive to obtain and maintain, this species is quite popular for experiments in the area of sepsis. For these reasons, in our experiments of sepsis the pig was chosen as a chronically instrumented large animal in a model of sepsis.

Endotoxin The toxic component of the bacterial wall of all gram-negative bacteria is called endotoxin. This endotoxin has a macromolecular glycolipid structure, which is termed lipopolysaccharide (LPS). LPS is able to provoke a generalized proinflammatory response in the infected host (15). In this thesis, the terms endotoxin and LPS will therefore be used interchangeably. The term endotoxemia will be used to denote the state induced by infusing LPS.

LPS is a stable and relative pure compound although the chemical composition varies from batch to batch. LPS is convenient to use because doses are readily measured and controlled. Gram-negative infections including LPS are detected in most of the suspected septic patients. LPS is a fundamental pathophysiological determinant in sepsis. Many similarities between sepsis and LPS-induced endotoxemia are observed including changes already mentioned in paragraph 1.1: "Clinical background". Therefore, the use of LPS-induced endotoxemia seems a reasonable paradigm for sepsis. We attempted to optimize the imitation of human sepsis and the related clinical syndrome in humans. Reasoning that in patients with sepsis, endotoxins are probably released into the circulation over an extended period, LPS from Escherichia coli subtype 055:B5 is delivered to the pigs by continuous intravenous infusion at the low rate of $3 \mu \mathrm{g} \cdot \mathrm{kg} \mathrm{bw}^{-1} \cdot \mathrm{h}^{-}$ ${ }^{1}$ for an extended period of $24 \mathrm{~h}$. Pigs were supported by a fluid infusion to compensate for hypovolemia in order to evoke a hemodynamic profile in which cardiac output and 
organ perfusion are well preserved. To reproduce the profile of a chronic human septic condition, endotoxin challenge was followed by enteral infusion of a liquid proteinenriched meal. This model of (post) endotoxemia provides a tool for studying metabolic alterations under and after sepsis-resembling conditions.

Among the disturbances of sepsis, one of the most important characteristics is the loss of lean body mass, especially muscle proteins. The nutritional status may be an important determinant in the response to stressed conditions such as sepsis. By choosing a proper control group of similar nutritional status, discrimination between the effects of starvation and those of sepsis for that reason can be made. Because alterations in patients with sepsis can be maintained for weeks, it is of importance to obtain a relevant model suitable for prolonged metabolic studies. Numerous models have been described, but most of them provide only short-term observations because the majority of the animals die within 24 to $48 \mathrm{~h}$.

\section{Methods}

\subsection{Pigs}

All experimental studies in this thesis were conducted in female crossbred (Yorkshire $x$ Dutch Landrace: $20-22 \mathrm{~kg}$ body weight (bw)) pigs. Pigs were housed individually in steel pens $(2 \mathrm{~m} \times 3 \mathrm{~m})$ with PVC coated floors, each equipped with an automated watering device. Pigs had ad libitum access to water. Each morning, the pigs were fed a proprietary diet (Zeugenkorrel Universeel Econ, Landbouwbelang, Roermond, the Netherlands), supporting a growth rate of approximately $300 \mathrm{~g}$ per day.

\subsection{Surgical procedure}

In order to measure blood flow and substrate fluxes across the organs, $80 \mathrm{~cm}$ long catheters (I.D. $1.0 \mathrm{~mm}$, O.D. $1.8 \mathrm{~mm}$, Tygon, Westvaco, Cleveland, $\mathrm{OH}$, USA) were inserted in the afferent and efferent blood vessels of the organ as indicated in Figure 1 during an operational procedure.

\subsection{Postoperative care}

Pigs wore a canvas harness to allow easy handling and to protect the catheters. The catheters were flushed every three days to maintain patency. One day after the operation, pigs were fed $100 \mathrm{~g}$, the second day $250 \mathrm{~g}$ and from the third day onwards, the pigs were fed $1 \mathrm{~kg}$ of food daily. When the pigs were recovered within 10 to 14 days, baseline studies began. 


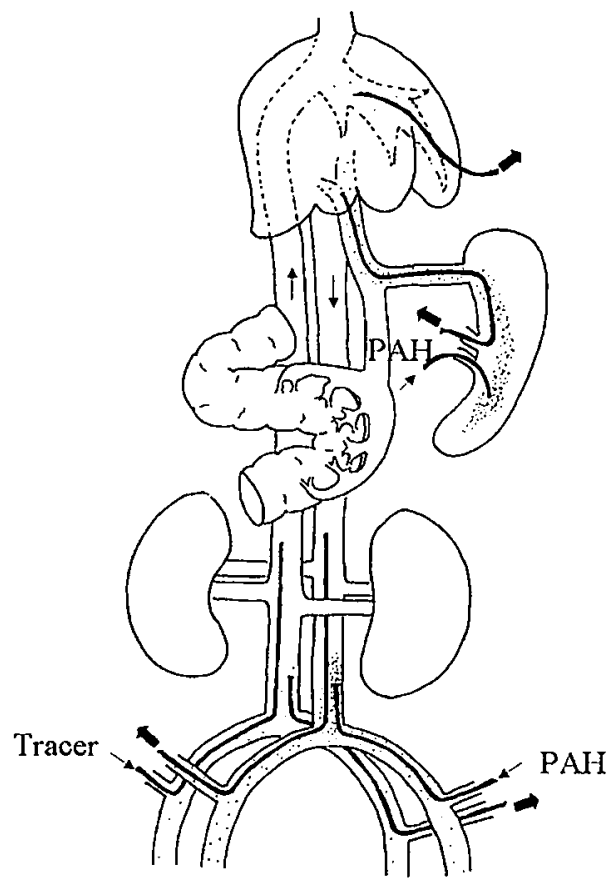

Figure 1. Catheter placement in the pig. PAH: para-amminohippuric acid is infused through the splenic vein, abdominal aorta for flow determination. Tracer is infused through the caval vein for turnover measurements. Blood is sampled from the abdominal aorta, caval vein, renal vein and portal vein.

Two catheters were inserted in the abdominal aorta; one catheter at a level just above the bifurcation (A1) and one at a level above the right renal vein (A2). Two catheters were placed in a similar way in the inferior caval vein ( $\mathrm{V} 1$ and $\mathrm{V} 2$ respectively). Catheters were also inserted in the renal $(R)$, portal $(P)$, hepatic $(H)$ and splenic vein $(S)$. In the experiment in which hemodynamics of the pigs were measured, a Swann-Ganz catheter was inserted via the inferior caval vein, via the right side of the heart into the pulmonary artery. The $A 1$ and the $S$ catheters were used to infuse para-aminohippuric acid (PAH), the $V 1$ catheter to infuse isotope and endotoxin infusion and the $A 2, P, H, V 1$ and $R$ catheters to sample blood. Also a gastrostomy catheter (I.D. $1.6 \mathrm{~mm}$, O.D. $4.8 \mathrm{~mm}$, Tygon) was inserted. All catheters were fixed to and tunneled through the abdominal wall and skin. Finally, a jejunal anostomosis was made $20 \mathrm{~cm}$ distal to the Ligament of Treitz (the change from duodenum to jejunum) by a Bishop-Koop anastomosis based on an end-to-site construction as shown in Figure 1 of Chapter 10. After transection, the proximal end of the jejunum is connected $10 \mathrm{~cm}$ from the distal end at the antimesenterial site by an anostomosis with a single layer running suture (PDS 4-0 synthetic polydioxanon; Ethicon, Norderstedt, Germany). The distal end is tunneled through the right abdominal wall. The distal end is fixed to the skin by several ligatures. To avoid leaking of gatrointestinal juices from the jejunum, the stoma is closed by a filled balloon catheter (Size Ch.16, 30-50 ml; Rüsch-Gold, Kernen, Germany). 


\subsection{Experimental protocol}

A liquid diet (Nutrison Steriflo High-Protein; osmolarity $255 \mathrm{mOsmol} / \mathrm{L}$; Nutricia B.V., Zoetermeer, the Netherlands) was enterally infused into the gastrostomy catheter via a swivel system connected to a pump at a rate equivalent to $0.3 \mathrm{~g}$ protein $\mathrm{kg} \mathrm{bw}^{-1} \cdot \mathrm{h}^{-1}$. This meal is a high-protein liquid meal for clinical use and has an optimal protein/energy ratio and contains all vitamins, minerals and other nutritional elements that meet the requirements of growing pigs (14). The enteral nutrition was infused for a period of 3 days. After overnight fasting, a small amount of endotoxin (lipopolysaccharide from Escherichia coli 055:B5; Sigma Chemical Co., St. Louis, MO, $3 \mu \mathrm{g} \cdot \mathrm{kg}$ $\left.\mathrm{bw}^{-1} \cdot \mathrm{h}^{-1}\right)$ is infused intravenously for a period of $24 \mathrm{~h}$. As a fluid support, saline is infused intravenously to evoke a hypermetabolic septic condition. Six $\mathrm{h}$ after cessation of the endotoxin infusion enteral nutrition is resumed. Blood samples are taken from the A2, P, H, V1 and $\mathrm{R}$ catheters from the control group and from the endotoxin-treated pigs either in the fed or in the conscious states. Protocols in which arginine supplementation is tested, arginine was infused at a rate of $5.3 \mu \mathrm{mol} \cdot \mathrm{kg}$ bw ${ }^{1} \cdot$ min $^{-1}$ aiming at a 3 -fold increase in plasma arginine flux. Intravenous arginine was started either $8 \mathrm{~h}$ before the start of endotoxin infusion or $8 \mathrm{~h}$ after the start of endotoxin infusion whereas intragastric arginine started after cessation of endotoxin infusion. The experimental setup is shown in Figure 2.

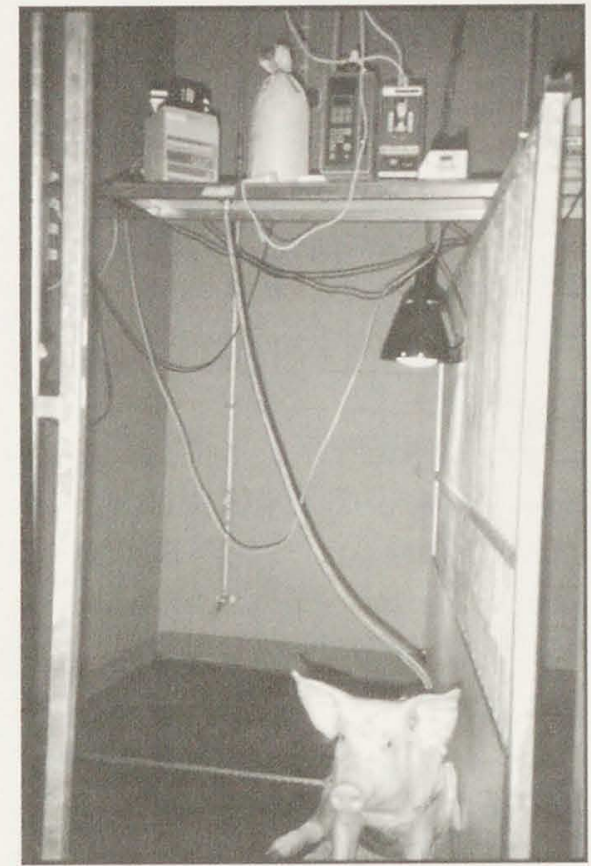

Figure 2. Pigs received a ready-to-use enteral diet via a swivel system connected to a pump.

\subsection{Infusion protocol}

Before the start of the isotopic infusions, background blood samples were taken. One hour before start of the endotoxin infusion, a prime $(5 \mathrm{~mL})$ followed by a continuous infusion $\left(40 \mathrm{~mL} \bullet \mathrm{h}^{-1}\right)$ of para-aminohippuric acid (PAH, $25 \mathrm{mmol} \bullet \mathrm{L}^{-1}$, A 1422, Sigma Chemicals CO, St. Louis, USA) was started through the $S$ and the A1 catheter. Immediately after the PAH bolus infusion, a primed and constant infusion of the 
radioactive or stable isotopes solutions was started via the V2 catheter. In plasma samples that were collected at 10-min intervals, the establishment of a steady state of the used isotopes was estimated by plotting the isotopic enrichment values in different sampling sites against time. A plateau of the isotopic enrichment of the used isotopes was observed during the last $60 \mathrm{~min}$ of the infusion protocol (17) when the calculated slope was not different from zero. Also, one hour after the start of the infusions, steady state conditions for PAH (11) were obtained. Blood samples were collected in triplicate at 15-min intervals, during the last 60 min of the isotope and PAH infusion.

\subsection{Cardiac output}

The insertion of a pulmonary artery catheter (Swan-Ganz catheter) allowed recording of cardiac function. Ice-cold infusions of saline were performed into the proximal infusion lumen of the Swan-Ganz catheter and CO measurements by the thermodilution method were obtained. Also, central temperature of the pig was monitored by Swan-Ganz.

\section{Measurement models of protein metabolism}

\subsection{Existing models}

A number of different methods are available for measuring protein synthesis and degradation (turnover) at whole body level and across the organs in vivo. (For thorough review of tracer techniques see: (6)). In vivo methods of protein synthesis can be classified in direct and indirect measurements.

\subsubsection{Direct measurements}

Direct methods for the measurement of protein synthesis rely upon the incorporation following the administration of an isotopically labeled precursor amino acid either as a flooding dose, or as a continuous infusion. The indirect measurement of protein synthesis is based upon measurements of plasma following a primed continuous infusion of a tracer. The methods that directly measure fractional protein synthesis involve measurements of the amount of isotope incorporated into protein of a tissue biopsy in time. In studies using the "flooding dose technique", a large dose of enriched substrate is injected, expanding the free amino acid pool several fold and "flooding" all intracellular pools. By measuring the incline in enrichment of tissue free amino acids and the enrichment of protein-bound amino acids at a relatively small time interval (several minutes), the proportion of protein replaced each day can be estimated. In the "continuous infusion" method, an isotope is infused for a relatively long period (several hours). At the end of the infusion period, when a steady state is reached, the enrichment of the isotope is determined in tissue protein and in the free tissue pool (reflecting tissue 
protein pool). Subsequently, the fractional synthesis rate of protein can be determined over a certain period of time. For the methods depending upon continuous infusion of labeled amino acid, the advantages include the use of labeled amino acids at true tracer doses (i.e. with no disturbance of metabolism).

\subsubsection{Indirect measurements of products}

Indirect techniques include measurements of nitrogen balance and 3-methylhistidine, which is a post-translational amino acid, providing an indirect estimate of overall nitrogen metabolism and skeletal myofibrillar protein breakdown. The mRNA or proteins involved in the protein breakdown route (e.g. ubiquitin or $20 \mathrm{~S}$ proteasome subunit) are also often used as a measure of protein breakdown.

\subsubsection{Indirect measurements of protein synthesis and degradation}

The most common used methods in current use for studying protein synthesis in vivo are based on "indirect measurements". When an arterial and a venous catheter are placed, no sampling of tissue protein is required. Indirect measurements of amino acid disposal and production are based on dilution of isotopically labeled amino acids across the organ. For this purpose, a primed and constant infusion of the precursor amino acid is performed. By measuring the arterio-venous enrichment differences, i.e. isotope net balances across the organ, an estimation of disappearance of the (essential) amino acid reflecting protein synthesis, can be made. When both tracer and tracee net balance are available, the amino acid production rate (reflecting protein degradation) by that organ can be estimated as well, since the amino acid flux is the net balance of protein synthesis and protein degradation. In extension to (both the direct and the indirect) protein synthesis measurements, arterio-venous measurements create the ability to protein breakdown rates as well.

\section{2. "Two-compartment" model}

In human or animal models, the placement of one catheter allows blood sampling at one site, and thus, measurement of amino acid concentration at whole body level. In case two catheters are placed, one in the afferent blood vessel of the organ and one in the efferent blood vessel of the organ, concentration differences across that organ can be measured. By simultaneously performing flow measurements, amino acid fluxes across the organ can be studied. Net balance studies of amino acids across an organ however only provide information on the net uptake or release (net protein anabolism or catabolism) and dynamic processes (disposal and production) can not be quantified. For example; increased net uptake of a substrate may be either caused by increased disposal, or by decreased production and vice versa. Extension of substrate (tracee) 
studies by the use of isotopes (tracers) enables measurements of amino acid disposal and production, i.e. protein turnover rates, at whole body level or across the organ. After a primed and constant infusion of a tracer, arterial plasma tracer dilution values serve to calculate protein degradation, which equals protein synthesis in steady state conditions. This is described as a "one-compartment" model. When both arterial and venous enrichments are available, the protein degradation can be calculated in addition to protein synthesis in a "two-compartment" model, the plasma and the interstitial fluid representing the two compartments (18). A "three-compartment" model can be applied when tissues or biopsies are available, the third compartment represents the free tissue pool. In the following paragraph the backgrounds of the "two-compartment" are outlined.

\subsubsection{Organ flow and flux measurements}

Flux measurement of a substrate across an organ involves both the measurement of plasma flow across the organ and the substrate concentration difference across the organ. The plasma flow measurements across the organs were performed in steady state conditions. Measurements were based on the simple dilution principle made by Fick that the concentration of the indicator downstream the place where the indicator enters the stream depends upon the flow rate. At steady state, the amount of indicator entering the stream equals the amount excreted. If the infusion rate and the concentration of the indicator up and downstream of the infusion site are known, the flow rate can be calculated. In other words, the infusion site of indicator is between the site of venous (upstream) and arterial (downstream) blood sampling. The concentration of indicator upstream depends on the blood flow through the organ.

During infusion of para-amino hippuric acid (PAH) indicator into a vessel at a constant rate $\left(1, \mathrm{nmol} \cdot \mathrm{kg} \mathrm{bw}^{-1} \cdot \mathrm{min}^{-1}\right)$ at steady state, the organ plasma flow can be calculated. The dilution of PAH across the organ was calculated as the concentration difference of PAH across the organ. Thus, flow $\mathrm{BLOOD}\left(\mathrm{mL} \cdot \mathrm{kg} \mathrm{bw}^{-1} \cdot \mathrm{min}^{-1}\right)$ was calculated using equation (1) in which [PAH $]_{V}$ and $[\mathrm{PAH}]_{A}$ are the concentrations of $\mathrm{PAH}$ in the venous and arterial blood, respectively. The blood flow was converted to plasma flow as in (2):

(1) Flow $_{\text {BLOOD }}=1 /\left([\mathrm{PAH}]_{V}-[\mathrm{PAH}]_{\mathrm{A}}\right)$

(2) FloW PLASMA $=$ flow $_{\text {BLOOD }} \times\{100 /(100-$ hematocrit $)\}$

To calculate the flux rate or net balance (NB, nmol $\left./ \mathrm{kg} \mathrm{bw}^{-1} \cdot \mathrm{min}^{-1}\right)$ of a substrate across an organ, the plasma flow is multiplied with the plasma venous concentration $[\mathrm{V}]$ minus arterial concentration [A] difference of the substrate (3). Therefore, a positive NB represents net release or efflux and a negative NB represents net uptake or influx of substrate across the organ.

$N B=$ flow $_{\text {PLASMA }} \times([\mathrm{V}]-[\mathrm{A}])$ 
Because blood was sampled from the $A 2, P, H, V 1$ and $R$ catheters, substrate fluxes across the portal-drained viscera (PDV), the splanchnic area, the hindquarter and the kidney could be made. The portal-drained organs were thought to mainly represent the intestinal tract including pancreas, stomach and spleen. The splanchnic area is the sum of the PDV and liver, therefore, calculations on liver are made by subtracting PDV from splanchnic values. Calculations on skeletal muscle kinetics are made by making the assumption that hindquarter represents $50 \%$ of the whole body muscle. Renal kinetics is calculated per two kidneys.

\subsubsection{Substrate turnover measurements}

Whole body kinetics To calculate production and disposal rates of a substrate on whole body level or across an organ, stable or radioactive enrichments in arterial and venous plasma are determined. The measurements of the radioactive enrichment of an isotope (specific activity; $\mathrm{SA}$ in $\mathrm{dpm} \cdot \mathrm{mL}^{-1}$ ) involves the chromatographic isolation of the desired component peak collection after HPLC separation and subsequent radioactivity counting of the amount of radioactivity accumulated in the fraction (16). To measure the stable tracer enrichment of an isotope (tracer to tracee ratio; TTR), a High Performance Liquid Chromatography (HPLC) system in connection to a Mass Spectrometry (MS) system (LCQ, Finnigan) was used. By means of this HPLC-MS technique the TTR of the different mass isotopes of the amino acid can be measured on-line by electronspray ionization and mass scanning after isolating the target amino acid by HPLC separation (17). Enrichment values were obtained by subtracting pre-tracer infusion background values.

For calculations of substrate (e.g. amino acid, glucose, urea) turnover rates, a plateau of the infused isotope is required. This means that the plasma pool of free isotope is in a steady state, i.e., that the amounts of isotope entering and leaving per unit of time are equal. The whole body substrate flux, i.e. the rate of appearance or disappearance of substrate from or into the free plasma pool is defined as $\mathrm{QRa}\left(\mathrm{nmol} \cdot \mathrm{kg} \mathrm{bw}^{-1} \cdot \mathrm{min}^{-1}\right)$ and determined from the equation (4). "l" Represents the rate of the tracer infused and $E_{A}$ the enrichment (TTR or SA) of the isotopic tracer in the arterial plasma.

$$
Q R a=1 / E_{A}
$$

Organ kinetics The arterial and venous concentration of tracer in the plasma are represented by are [a] and [V] and are calculated in (5) in which $E_{A}$ and $E_{V}$ are the arterial and venous enrichments of the isotopic tracer, respectively. The net balance of an isotopic tracer across the organ, $\mathrm{nb}$, was calculated by formula (6) using $a-v$ tracer concentration differences.

$$
[\mathrm{a}]=\mathrm{E}_{\mathrm{A}} \times[\mathrm{A}] \text { or }[\mathrm{V}]=\mathrm{E}_{\mathrm{V}} \times[\mathrm{V}]
$$




$$
\mathrm{nb}=\text { flow } \times([\mathrm{a}]-[\mathrm{v}])
$$

Calculations on organ substrate turnover, i.e., disposal and production rates are based on the tracer disappearance in that organ. The rate of disposal $\left(\mathrm{nmol} \cdot \mathrm{kg} \mathrm{bw}^{-1} \cdot \mathrm{min}^{-1}\right)$ of substrate represents its total rate of metabolism (i.e. disappearance in a macromolecule plus breakdown) and is calculated in (7) dividing the tracer $\mathrm{nb}$ by its precursor pool, the venous enrichment (which is thought to approach best the intracellular enrichment of the organ). If the infused isotope concerns an essential amino acid that can not be newly synthesized, disposal and production of this amino acid reflect protein synthesis and degradation, respectively.

\section{(7) $\quad$ Disposal $=n b / E_{V}$}

Since the NB of substrate or tracee across an organ is the net difference between production and disposal, the production is denoted in (8):

(8) Production $=$ NB + Disposal

\subsubsection{Substrate de novo synthesis measurements}

Whole body kinetics The plasma isotope transfer from $\mathrm{N}_{2}$-arginine to $\mathrm{N}_{1}$-citrulline represents de novo NO synhtesis. Similarly, the plasma isotope transfer from $D_{5}$ phenylalanine to $D_{4}$-tyrosine represents newly formed tyrosine from phenylalanine hydroxylation. Arginine de novo synthesis can be calculated from $C_{1} D_{2}$-citrulline to $C_{1} D_{2}$ arginine conversion. The rate of conversion of a substrate $\left(S\right.$; e.g. $\mathrm{N}_{2}$-arginine, $D_{5}$ phenylalanine or $C_{1} D_{2}$-citrulline) into its metabolic product ( $P$; e.g. $N_{1}$-citrulline, $D_{4}$ tyrosine or $C_{1} D_{2}$-arginine, respectively) is expressed as $Q_{S \rightarrow P}$ and is calculated as in formula (9).

$$
\text { (9) } Q_{S \rightarrow P}=Q \operatorname{Rap} \times\left(E_{P} / E_{S}\right) \times Q R a_{S} /\left(I_{S}+Q_{S}\right)
$$

$E_{P}$ and $E_{S}$ represent enrichments (in TTR) of the metabolic product and substrate, respectively, in the arterial plasma. $Q R a_{p}$ and $Q R a_{s}$ are the whole body rate of appearance of the product and of the substrate, respectively. The whole body appearance of the product ( $Q R a_{p}$ ) of interest (e.g. citrulline, tyrosine or arginine) can be measured by using an isotopomere, i.e. an isotope, which has the same molecular structure as the metabolic product but differs from it in molecular mass (e.g. $C_{1} D_{2}$ citrulline, $D_{2}$-tyrosine or $\mathrm{N}_{2}$-arginine). The expression $Q R_{\mathrm{S}} / \mathrm{I}_{\mathrm{S}}+\mathrm{Q}_{\mathrm{S}}$ is adjusted to correct for the contribution of infused substrate $\left(I_{S}\right)$ to $Q$ Rap.

Organ kinetics Calculations on the organ de novo synthesis rate of NO, tyrosine or arginine are based on the same principle of isotopic conversion used for whole body calculations. The organ tracer net balance of the isotopic metabolic product ( $\mathrm{nb}_{\mathrm{p}} ; \mathrm{N}_{1}$ citrulline, $\mathrm{D}_{4}$-tyrosine or $\mathrm{C}_{1} \mathrm{D}_{2}$-arginine) must, however, be corrected for its loss by 
extraction across the organ. A good approximation of this fractional extraction (FE $\mathrm{F}_{\mathrm{P}}$ ) can be obtained by means of calculating the partial dilution of the isotopomere (10).

$$
\text { (10) } \quad F E_{P}=([a]-[v]) /[a]
$$

The term $1-F E_{P}$ represents the fraction of total product that bypasses metabolism in the organ and so appears in the output and is used for correction of $n b_{p}(11)$ :

$$
n b_{p}=\text { flow } \times\{[v]-[a] \times(1-F E)\} p
$$

By using this corrected $n b_{p}$, the organ de novo synthesis (Organ $s \rightarrow p$ ) of the metabolic product (NO, tyrosine or arginine) can be calculated similar to disposal in (12) in which the arterial substrate enrichment $E_{A} s$ is used as precursor pool because the upstream compartment is thought to best reflect the intracellular enrichment.

(12) Organ ${ }_{S \rightarrow p}=n b_{P} / E_{A s}$

\section{References}

1. Bohley, P., J. Kopitz, G. Adam, B. Rist, F. von Appen, and $S$. Urban. Post-translational arginylation and intracellular proteolysis. Biomed Biochim Acta 50: 343-6, 1991.

2. Brackett, D. J., C. F. Schaefer, P. Tompkins, L. Fagraeus, L. J. Peters, and M. F. Wilson. Evaluation of cardiac output, total peripheral vascular resistance, and plasma concentrations of vasopressin in the conscious, unrestrained rat during endotoxemia. Circ Shock 17: 273-84, 1985.

3. Dodds, W. J. The pig model for biomedical research. Fed Proc 41: 247, 1982.

4. D'Orio, V., C. Wahlen, L. M. Rodriguez, A. Fossion, J. Juchmes, J. Halleux, and $R$. Marcelle. A comparison of Escherichia coli endotoxin single bolus injection with low-dose endotoxin infusion on pulmonary and systemic vascular changes. Circ Shock 21: 207-16, 1987.

5. Fink, M. P., and S. O. Heard. Laboratory models of sepsis and septic shock. J Surg Res 49: 186-196, 1990.

6. Hasselgren, P. O., P. P. S. HC, W. BW, and F. JE. Methods for studying protein synthesis and degradation in liver and skeletal muscle. $J$ Surg Res 45: 389-415, 1988.

7. Hinshaw, L. B., L. A. Solomon, D. D. Holmes, and L. J. Greenfield. Comparison of canine responses to Escherichia coli organisms and endotoxin. Surg Gynecol Obstet 127: 981-8, 1968.
8. Hussain, S. N., and C. Roussos. Distribution of respiratory muscle and organ blood flow during endotoxic shock in dogs. I Appl Physiol 59: 1802-8, 1985.

9. Hynninen, M., M. Valtonen, H. Markkanen, $M$. Vaara, P. Kuusela, I. Jousela, A. Piilonen, and O. Takkunen. Intramucosal $\mathrm{pH}$ and endotoxin and cytokine release in severe acute pancreatitis. Shock 13: 79-82, 2000.

10. Lang, C. H., G. J. Bagby, G. H. Bornside, L. J. Vial, and J. J. Spitzer. Sustained hypermetabolic sepsis in rats: characterization of the model. J Surg Res 35: 201-10, 1983.

11. Merimee, T. J., D. A. Lillicrap, and D. Rabinowitz. Effect of arginine on serum-levels of human growth-hormone. Lancet 2: 668-70, 1965.

12. Miller, E. R., and D. E. Uilrey. The pig as a model for human nutrition. Annu Rev Nutr 7: 361-82, 1987.

13. Platell, C., R. McCauley, R. McCulloch, and J. Hall. The influence of parenteral glutamine and branched-chain amino acids on total parenteral nutrition-induced atrophy of the gut. JPEN J Parenter Enteral Nutr 17: 348-354, 1993.

14. Pond, W. G. Life cycle feeding: maternal nutrition and progency development. In: Swine in biomedical research, edited by I. M. E. Tumbleson Plenum press, New York, 1986, p. 915-930. 
15. Qureshi, S. T., P. Gros, and D. Malo. The Lps locus: genetic regulation of host responses to bacterial lipopolysaccharide. Inflamm Res 48: 613-20., 1999.

16. Van Eijk, H. M. H., M. P. L. Huinck, D. R. Rooyakkers, and N. E. P. Deutz. Automated simultaneous isolation and quantification of labeled amino acid fractions from plasma and tissue by ion-exchange chromatography. $J$ Chromatogr., 1994.
17. Van Eijk, H. M. H., D. R. Rooyakkers, and N. E. P. Deutz. Determination of amino acid isotope enrichment using liquid chromatography-mass spectrometry. Anal Biochem 271: 8-17, 1999.

18. Wolfe, R. R. Radioactive and stable isotope tracers in biomedicine. Principles and practice of kinetic analysis. New York: Wiley-Liss, 1992. 
CHAPTER 3

\title{
ALTERATIONS IN ORGAN PROTEIN TURNOVER AND SUBSTRATE FLUXES DURING ENDOTOXEMIA
}

\author{
Maaike J Bruins \\ Wouter H Lamers * \\ Nicolaas EP Deutz \\ Peter B Soeters
}

Department of Surgery, Maastricht University, The Netherlands

-Department of Anatomy \& Embryology, University of Amsterdam, AMC, The Netherlands 


\section{Abstract}

Infection and sepsis evoke a state of catabolism implying that the body exhibits net protein loss. Although it is clinically evident that muscle protein is lost, protein dynamics in central organs like liver and gut are poorly quantified. It is also unknown whether changes in protein kinetics are effected by changes in protein synthesis or in protein degradation.

As a model of hyperdynamic sepsis, post-absorptive pigs received intravenous endotoxin infusion over $24 \mathrm{~h}$. To study portal-drained viscera (zintestine), liver and hindquarter ( $\approx$ muscle) metabolism, pigs were catheterized. Plasma samples were obtained before and during the 24-h endotoxin infusion. Protein synthesis and degradation were simultaneously measured across the organs using radioactive isotopes.

During starvation, protein breakdown and amino acid degradation decreased in liver and remained unchanged in muscle and gut. Whereas intestinal protein turnover remained unaffected during endotoxemia, muscle protein breakdown doubled thereby exceeding increased liver protein synthesis with resultant net protein loss of the whole body. Muscle contributed to gluconeogenic substrate flux to the liver by providing branched-chain amino acid (BCAA), glutamine, glutamate, alanine and lactate. The portal-drained viscera also importantly contributed to gluconeogenic substrate provision by increased lactate and glycine efflux. This ultimately results in increased hepatic substrate uptake, not only serving protein, but also glucose, glutamate and urea synthesis.

In starvation, reduced organ protein breakdown and amino acid transamination conserve nitrogen and carbon, whereas during endotoxemia, these processes are accelerated, the carbon and nitrogen between organs effectively being cycled.

\section{Introduction}

One of the most critical features of sepsis is the loss of lean body mass, predominantly resulting from muscle catabolism. Over the past view years, new strategies in nutritional support aiming at maintenance of lean body mass have been developed. Despite the large amounts of carbohydrate, fat and protein or amino acids that are supplied to critically ill patients, loss of lean body mass invariably persists $(21,34)$. It is for that reason of major importance to define organ substrate requirements that occur in response to critical illness such as sepsis. More insight into substrate metabolism in these "defense" organs will help to compose nutritional regiments that better support host defense and limit muscle catabolism. 
The complex integrated organ response serves to provide nutrients to the inflammatory cells to generate energy and proteins which assist in the initial host defense response and the later reparative processes. The main feature of sepsis is the increased transfer of substrate from periphery to central organs which play crucial roles in host defense response like the gut and the spleen (6).

It is generally accepted that endotoxins play an important role in the metabolic response to sepsis since they induce an array of responses affecting protein metabolism in a way similar to sepsis. The nutritional status is an important determinant in the response to stressed conditions (31), emphasizing the necessity of a proper control group with similar nutritional status.

The profound alterations in protein and glucose metabolism that occur in the muscle under septicemic conditions are well described. However, because the hepatic and portal vein are not easily accessible, knowledge about the in vivo protein and amino acid metabolism in liver and gut during endotoxemia is relatively scarce. In fact, a number of in vivo studies have dealt with organ protein synthesis during endotoxemia whereas only few animal studies have described simultaneous changes in protein degradation. Moreover, most experiments dealing with the modifications in protein metabolism in various tissues were performed in severe models of sepsis using high dose primed injections of bacteria or endotoxin eliciting a hypodynamic response with characteristic low organ blood flow (14). To reproduce a hyperdynamic sepsis profile more characteristic of the human situation, in which cardiac output and organ perfusion are maintained by fluid resuscitation, endotoxin was infused for an extended period in parallel with generous fluid resuscitation. The exact manner in which protein metabolism of three of the main organs, the hindquarter ( $\approx$ muscle), the portal-drained viscera ( $\approx$ gut) and the liver, is changed in terms of modifications in protein synthesis and degradation, remains to be elucidated. Also, changes in the relative contribution of amino acid and glucose/lactate fluxes of these organs occurring during endotoxemia remain to be assessed. Catheter cannulation together with dye-isotope infusion protocols allowed us to measure protein synthesis and degradation rates across the individual organs, and to assess the substrate flux utilized by individual organs in this hyperdynamic sepsis model.

\section{Materials and methods}

Crossbred pigs (female, Yorkshire $\times$ Dutch Landrace, 20-22 kg body weight, $\mathrm{n}=14$ ) were individually housed receiving $1 \mathrm{~kg}$ of regular pig feed (Landbouwbelang, Roermond, The Netherlands; $16 \%$ crude protein) daily, supporting a growth rate of approximately $300 \mathrm{~g}$ per day. The Animal Ethics Committee of the Maastricht University approved the study. 


\section{Surgical Procedure}

After an overnight fast, all animals were anesthetized with a mixture of $\mathrm{N}_{2} \mathrm{O} / \mathrm{O}_{2}(1: 2)$ and halothane $(0.8 \%$ ), and intubated. Pigs were administered (i.v.) $6.25 \mathrm{mg} / \mathrm{kg}$ body weight (bw) lincomycin.2HCl (Lincomycin, A.U.V., Cuyk, The Netherlands) as bactericidal prophylaxis and $12.5 \mathrm{mg} / \mathrm{kg}$ bw spectinomycin.HCl lyophil (Spectinomycin, A.U.V.) as bacteriostatic prophylaxis. To avoid coagulation and to provide postoperative analgesia, $50 \mathrm{mg} / \mathrm{kg}$ bw flunixine (Finadyne, Schering-Ploegh, Brussel, Belgium) was given. During surgery, the anesthesia was maintained by the $\mathrm{N}_{2} \mathrm{O} / \mathrm{O}_{2}$-halothane mixture. A midline laparotomy was performed and catheters were implanted as reported previously (11). In summary, 7 vessels were cannulated. Two catheters were inserted into the abdominal aorta: one at a level just above the bifurcation (A1) and one at a level above the right renal vein (A2); similarly, two catheters were inserted into the inferior caval vein (V1 and $\mathrm{V} 2$, respectively). Furthermore, catheters were inserted in the portal $(P)$, hepatic $(H)$ and splenic (S) vein. The $A 1$ and the $S$ catheters were used for the infusion of paraaminohippuric acid (PAH) for determination of the plasma flow and the V1 catheter for isotope and endotoxin infusion. For portal-drained viscera, splanchnic and hindquarter flux measurements, blood sampled from the $P, H$ and $V 1$ catheters, was used, respectively. A gastrostomy catheter was inserted into the stomach to enable infusion of a liquid diet. All catheters were tunneled through the abdominal wall and skin.

The pigs wore a canvas harness to protect the catheters and stoma, and to allow easy handling. The first day post-operatively, the pigs were fed $100 \mathrm{~g}$, the second day $200 \mathrm{~g}$ and from the third day onwards, $1 \mathrm{~kg}$ of food daily. To avoid catheter tip infections and to maintain patency, catheters were regularly flushed with $150 \mathrm{mmol} / \mathrm{L} \mathrm{NaCl}$ (saline) and filled with a solution containing a mixture of gentamycin ( $20 \mathrm{~g} / \mathrm{L}$, Gentamycin $5 \%$, A.U.V.) and chymotrypsin (0.225 U/L, Merck, Darmstadt, Germany) in saline. Ten days after surgery, to standardize daily food intake, pigs were given continuous liquid enteral nutrition via the gastrostomy catheter for a period of 4 days. The liquid diet was prepared by mixing $4 \mathrm{~L}$ of a liquid diet $(63 \mathrm{~g} / \mathrm{L}$ protein, Nutrison Steriflo High-Protein, Nutricia, Zoetermeer, The Netherlands) and $1 \mathrm{~L}$ of tap water.

\section{Experimental protocol}

The night before experiments (12 PM), enteral nutrition was ceased. The next morning (8 $A M)$, the pigs were placed in a cage and experiments were conducted. Seven pigs received $3 \mu \mathrm{g} \cdot \mathrm{kg} \mathrm{bw}^{-1} \cdot \mathrm{h}^{-1}$ lipopolysaccharide endotoxin ( $E$. coli serotype: 055:B5, Sigma Chemicals Co., St. Louis, USA) dissolved in saline during $24 \mathrm{~h}$ via the V2 catheter. Seven control animals received saline at an equivalent infusion rate. All pigs received extra saline to secure replenishment of intravascular volume losses: $30 \mathrm{~mL} \cdot \mathrm{kg} \mathrm{bw}^{-1} \cdot \mathrm{h}^{-1}$ during the first $8 \mathrm{~h}$ and $20 \mathrm{~mL} \cdot \mathrm{kg} \mathrm{bw}^{-1} \cdot \mathrm{h}^{-1}$ the following $16 \mathrm{~h}$. Samples were taken before 
(Con0 group) and $24 \mathrm{~h}$ after the start of endotoxin (ET24 group) or saline (Con24 group) infusion. Rectal temperature of the pig was measured daily.

\section{Sample processing}

Promptly after sampling, blood was distributed in heparinized tubes (Sarstedt, Nümbrecht, Germany) on ice. For blood gas determination (arterial pH, bicarbonate, $\mathrm{P}_{\mathrm{O} 2}$ and $P_{\mathrm{CO} 2}$ ), $0.2 \mathrm{~mL}$ heparinized blood was immediately analyzed on an automatic blood gas system (Acid Base Laboratory, Radiometer, Copenhagen, Denmark). Centrifugation was performed at $4^{\circ} \mathrm{C}$ for $5 \mathrm{~min}$ at $8,500 \mathrm{~g}$, plasma collected and kept on ice. Plasma processing for determination of $\mathrm{PAH}$, glucose, lactate and amino acids was performed as previously described (10). All samples were stored at $-80^{\circ} \mathrm{C}$ until further analysis.

\section{Biochemical analysis}

PAH was detected spectrophotometrically by standard enzymatic methods as described before (12). Plasma amino acid and glucose and lactate concentrations were determined by a fully automated High Performance Liquid Chromatography system (HPLC, Pharmacia, Woerden, The Netherlands), using methods previously reported $(38,39)$. Phenylalanine, valine and glucose fractions were collected and counted for radioactivity on a liquid scintillation spectrophotometer to determine the specific activity. Insufficient radioactivity was detected in the fractions of the pre-tracer background blood samples to reliably calculate specific activity. The measurements of the acute-phase proteins haptoglobin, fibrinogen and $\alpha-1$ antitrypsin were performed in plasma and carried out on a Nephelometer BN 100 (Dade Behring Vertriebs $\mathrm{GmbH}$ and Co., Germany). Proteins were determined using rabbit anti-human haptoglobin, fibrinogen and $\alpha-1$ antitrypsin antibodies (Dade-Behring) as performed for human plasma samples. Standard curves for haptoglobin and fibrinogen were constructed using a purified human (Dade-Behring) and a secondary porcine standard (Sigma Chemicals Co., St. Louis, MO, USA). High correlation coefficients $(>0.9)$ were found for the results of both methods. Because a porcine standard for $\alpha-1$ antitrypsin was not available, protein concentrations were expressed in human units.

\section{Infusion protocol}

On the morning of the trial, one hour before start of the endotoxin infusion, a primed infusion protocol was conducted. An infusion of para-aminohippuric acid (PAH; $25 \mathrm{mM}, \mathrm{A}$ 1422, Sigma Chemicals Co.) was started at a rate of $40 \mathrm{~mL} / \mathrm{h}$ per catheter through the $S$ and the $A 1$ catheter, after an initial bolus of $5 \mathrm{~mL}$ (36). Directly thereafter, a priming dose $\left(1 \mu \mathrm{Ci} \cdot \mathrm{kg} \mathrm{bw}^{-1}\right)$ followed by a constant infusion $\left(1 \mu \mathrm{Ci} \cdot \mathrm{kg} \mathrm{bw}^{-1} \cdot \mathrm{h}^{-1}\right)$ of $\mathrm{L}-[2,6-$ $\left.{ }^{3} \mathrm{H}\right]$ phenylalanine and $\mathrm{L}-\left[3,4-{ }^{3} \mathrm{H}\right]$ valine (Amersham, Buckinghamshire, UK) and a primed 
$\left(0.5 \mu \mathrm{Ci} \cdot \mathrm{kg} \mathrm{bw}^{-1}\right)$ constant infusion $\left(0.5 \mu \mathrm{Ci} \cdot \mathrm{kg} \mathrm{bw}^{-1} \cdot \mathrm{h}^{-1}\right)$ of $\mathrm{D}-\left[6-{ }^{3} \mathrm{H}\right] \mathrm{glucose}$ (NEN Dupont, NET-100A, Mechelen, Belgium) was given via the V2 catheter. Also via this catheter, a primed infusion of $L-\left[{ }^{15} \mathrm{~N}_{2}\right]$ arginine was started, the results of which will be published elsewhere. Before the start of the infusions, background blood samples were taken. One hour after the start of the infusions, steady state conditions for PAH (data not shown) were obtained. Isotopic plateau of $\mathrm{L}-\left[2,6-{ }^{3} \mathrm{H}\right]$ phenylalanine, $\mathrm{L}-\left[3,4-{ }^{3} \mathrm{H}\right]$ valine and $\mathrm{D}-[6-$ ${ }^{3} \mathrm{H}$ ]glucose was observed during the last $60 \mathrm{~min}$ of the infusion protocol in collected samples (10). This was assessed based on the observation that when isotopic enrichment values in different sites were plotted against time, the ensuing slopes were not different from zero. Blood samples were collected in triplicate at 15 -min intervals, starting the last $60 \mathrm{~min}$ of the isotope and PAH infusion.

\section{D-[6- $\left.{ }^{3} \mathrm{H}\right]$ glucose kinetics}

The whole body glucose flux was determined with $D-\left[6-{ }^{3} \mathrm{H}\right]$ glucose infusion. The value for $Q$ glucose represents the sum of glucose production from glycogen breakdown and gluconeogenesis. However, $6{ }^{3} \mathrm{H}$ label may be lost to some extent $(\sim 10 \%)$ in the phosphoenolpyruvate cycle which contributes to a corresponding overestimation of the measured whole body glucose flux $(44,47)$.

\section{$L-\left[2,6-{ }^{3} H\right] p h e n y l a l a n i n e$ and $L-\left[3,4-{ }^{3} H\right]$ valine kinetics}

Whole body appearance of phenylalanine calculated by means of $\mathrm{L}-\left[2,6-{ }^{3} \mathrm{H}\right]$ phenylalanine isotope was used as an indication of whole body protein breakdown since this amino acid is considered not to be newly synthesized. In the muscle and the gut, the phenylalanine disposal is a reflection of protein synthesis and phenylalanine production of breakdown, as in these organs phenylalanine breakdown is low (37). Valine disposed in gut and muscle tissue can either be used in protein synthesis or become metabolized. Valine can be transaminated to its $\alpha$-keto acid which can be further oxidized (16) although the rate of oxidation of valine in these organs is expected to be low during starvation (23). In the liver, valine is generally considered to be mainly used for protein synthesis and not to be degraded since liver has a low transamination activity $(24,27,28)$. Valine production by the liver, therefore, can only originate from protein breakdown. Disposal of phenylalanine by the liver is a combination of protein synthesis and hydroxylation of phenylalanine to tyrosine (37). The rate of metabolized valine or phenylalanine was estimated from the assumption that the valine to phenylalanine molecular ratio in protein of the hindquarter, portal-drained viscera and liver of the pig equals $1.7(49)$. 


\section{Calculations}

The whole body flux or rate of appearance ( $\mathrm{Q} \mathrm{Ra}, \mu \mathrm{mol} \cdot \mathrm{kg} \mathrm{bw}^{-1} \cdot \mathrm{min}^{-1}$ ) of glucose and phenylalanine was derived from formula (1). I represents the rate of the tracer infused $\left(\mathrm{dpm} \cdot \mathrm{kg} \mathrm{bw}^{-1} \cdot \mathrm{min}^{-1}\right)$ and $\mathrm{SA}_{A}$ the specific activity of the isotopic tracer in the arterial plasma $\left(\mathrm{dpm} \cdot \mathrm{nmol}^{-1}\right)(47)$.

(1) $\quad \mathrm{QRa}=1 / \mathrm{SA}_{\mathrm{A}}$

The portal-drained organs mainly represent the gut. The splanchnic area is the sum of the portal-drained viscera and liver. Calculations on the liver are, therefore, made by subtracting portal-drained viscera from splanchnic values. Substrate metabolism across the hindquarter, the portal-drained viscera and the liver was calculated in a twocompartment model as described previously (47). Calculations on the kinetics in muscle were made on the assumption that the hindquarter represents $50 \%$ of whole body muscle (13). The plasma flow rates across the liver, portal-drained viscera and hindquarter were calculated using equation (2). This equation is based on the principle of indicator dilution methods in which I represents the rate of the PAH infused and $[\mathrm{PAH}]_{\mathrm{V}}$ and $[\mathrm{PAH}]_{\mathrm{A}}$ the concentration of $\mathrm{PAH}$ in the venous and arterial blood of the measured organ, respectively.

(2) FloW $_{B L O O D}=1 /\left([\mathrm{PAH}]_{V}-[\mathrm{PAH}]_{\mathrm{A}}\right)$

The blood flow was converted to plasma flow by using the equation (3):

(3) FloW PLASMA $=$ flow BLOOD $\times 100 /(100$ - hematocrit $)$

The substrate net balance was defined as NB and calculated in (4) by multiplying the mean plasma flow by the difference between $[\mathrm{V}]$ and $[A]$ that represent the venous and arterial plasma concentration of the amino acid, respectively.

$$
N B=\text { flow PLASMA } \times([V]-[A])
$$

Therefore, a positive NB represents net release or efflux and a negative NB represents net uptake or influx of substrate across the organ. The tracer net balance (nb) was calculated similarly by formula (5) using the arterial and venous tracer concentrations in the plasma, in which $S A_{A}$ and $S A_{V}$ represent the arterial and venous specific activity of the tracer, respectively.

$$
\left.n b=\text { flow } \times\left(S A_{A} \times[A]-S A_{V} \times M\right]\right)
$$

The rate of disposal of the amino acid represents the total rate of metabolism (amino acid degradation plus incorporation into protein) and is calculated by (6):

(6) $\quad$ Disposal $=n b / S A_{V}$ 
The $S A_{v}$ was thought to approach best the intracellular enrichment of the organ (2), and, therefore, used as precursor pool. Since the NB of substrate across an organ is the difference between production and disposal, the production is denoted as in (7):

$$
\text { (7) } \text { Production }=\text { NB }+ \text { Disposal }
$$

Alpha-amino nitrogen (AA) represents the sum of measurable $\alpha$-amino acids ${ }^{1}$ and GNAA the sum of the gluconeogenic $\alpha$-amino acids (all amino acids occurring in protein except for leucine and lysine). Branched-chain amino acids (BCAA) were calculated as the sum of valine, leucine and isoleucine.

\section{Statistics}

Results are presented as means \pm SEM. Because results at day 0 were similar in the Con and the endotoxin group, pigs were pooled into one Cono group. The data were subjected to a two-way analysis of variance (ANOVA) to assess group effects (endotoxin infusion), time effects (effect starvation) and interactions between group and time. Levels of significance were set at $P<0.05$.

Table 1. Temperature, arterial $\mathrm{P}_{\mathrm{CO} 2}$ and $\mathrm{PO}_{\mathrm{O}}$, hematocrit, bicarbonate $\left(\mathrm{HCO}_{3}{ }^{-}\right), \mathrm{O}_{2}$ saturation and organ plasma flow. Post-absorptive pigs (Con0) were either treated with saline (Con24) or endotoxin (ET24) during 24-h of fasting and fluid resuscitation.

\begin{tabular}{lrrr}
\hline & \multicolumn{2}{c}{ Con0 } & \multicolumn{2}{c}{ Con24 } & ET24 \\
\hline Temperature $\left({ }^{\circ} \mathrm{C}\right)$ & $37.9 \pm 0.2$ & $37.6 \pm 0.3^{\circ}$ & $39.9 \pm 0.2^{\ddagger 5}$ \\
Hematocrit & $28.9 \pm 0.6$ & $27.9 \pm 1.0^{\circ}$ & $24.5 \pm 0.8^{\dagger 8}$ \\
$\mathrm{pH}$ & $7.4 \pm 0.1$ & $7.3 \pm 0.1$ & $7.4 \pm 0.1$ \\
$\mathrm{P}_{\mathrm{CO}}(\mathrm{kPa})$ & $5.7 \pm 0.2$ & $5.2 \pm 0.6^{\circ}$ & $4.7 \pm 0.1^{\dagger}$ \\
$\mathrm{P}_{\mathrm{O} 2}$ & $12.6 \pm 0.5$ & $11.9 \pm 0.8$ & $13.0 \pm 0.8$ \\
$\mathrm{HCO}_{3}^{-}(\mathrm{mM})$ & $27.5 \pm 1.0$ & $21.8 \pm 3.2$ & $24.1 \pm 1.1$ \\
$\mathrm{O}_{2}$ saturation $(\%)$ & $97.2 \pm 0.3$ & $96.7 \pm 0.9$ & $97.2 \pm 0.4$ \\
Flow HQ (mL-kg bw $\left.{ }^{-1} \cdot \mathrm{min}^{-1}\right)$ & $28.1 \pm 3.6$ & $31.7 \pm 7.2$ & $42.6 \pm 4.4$ \\
Flow portal-drained viscera & $33.5 \pm 3.5$ & $26.3 \pm 4.2$ & $34.0 \pm 1.5^{\dagger}$ \\
Flow liver & $45.7 \pm 4.4$ & $48.1 \pm 4.4^{\star}$ & $55.9 \pm 4.3^{\dagger}$ \\
\hline
\end{tabular}

Data are mean \pm SEM. HQ: hindquarter. Statistical analysis by Two-Way ANOVA: ${ }^{*} P<0.05,{ }^{\circ} \mathrm{P}<0.01$; starvation plus infusion effect, ${ }^{\dagger} P<0.05,{ }^{\ddagger} P<0.01$; endotoxin effect and ${ }^{8} P<0.05,{ }^{5} P<0.01$; starvation $X$ endotoxin effect.

\footnotetext{
${ }^{1}$ GLU, ASN, SER, GLN, HIS, GLY, THR, CIT, ARG, ALA, TAU, TYR, VAL, MET, ILE, TRP. PHE, ORN, LEU, LYS
} 


\section{Results}

\section{Effect of 24-h starvation}

Table 1 shows the different hemodynamic parameters before fasting and after fasting and fluid (saline) resuscitation in the 24 -h endotoxin-treated pigs and control pigs. The body temperature, hematocrit and arterial $\mathrm{P}_{\mathrm{CO} 2}$ decreased upon fasting and saline infusion. Organ plasma flows did not change, except that of the liver, which increased by a 24-h fasting period. As can be observed from Table 2, the arterial concentration of urea decreased after 24-h fasting and saline infusion. Also, concentrations of all amino acids decreased as a consequence of fasting and fluid infusion, except for phenylalanine, which increased significantly. Figure $1 \mathrm{~A}$ shows that the whole body appearance of glucose remained unchanged after fasting. The whole body appearance of valine (Figure 1C) significantly decreased (16\%), whereas appearance of phenylalanine (Figure 1B) reflecting protein breakdown did not change as consequence of fasting.

A. Glucose
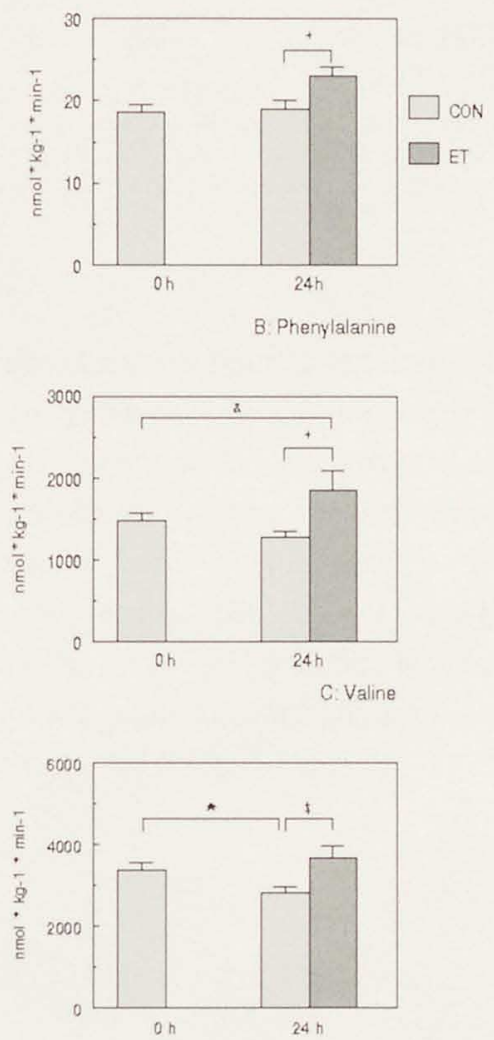

Figure 1. Whole body appearance of $A$ : glucose, $B$ : phenylalanine and $\mathrm{C}$ : valine in the postabsorptive state before (ConO) and $24 \mathrm{~h}$ after the start of saline (Con24) or endotoxin (ET24) infusion. Statistical analysis by Two-Way ANOVA: ${ }^{\star} p<0.05$; starvation plus infusion effect, ${ }^{\dagger} p<0.05,{ }^{\ddagger} p<0.01$; endotoxin effect and ${ }^{\&}<0.05$; starvation $\times$ endotoxin effect.

\section{Hindquarter}

Fasting enhanced BCAA, GNAA and total amino acid influx into the hindquarter area (Table 3). The increased amino acid influx was primarily accounted for by decreased alanine and glutamine efflux. As assessed by phenylalanine isotope, the starvation period did not significantly affect the protein synthesis but reduced protein breakdown thereby enhancing net protein anabolism across the hindquarter (Figure 2A). The contribution of hindquarter protein degradation to whole body protein degradation calculated by phenylalanine isotope amounted to $35 \%$ in both the Con0 and Con24 pigs. The estimated rate of valine not being used in protein synthesis was not changed in the time course of starvation (490 vs. 430 $\mathrm{nmol} \cdot \mathrm{kg} \mathrm{bw}^{-1} \cdot \mathrm{min}^{-1}, \mathrm{P}>0.05$ ). 
Table 2. Arterial concentrations in the postabsorptive state before (Con0) and $24 \mathrm{~h}$ after the start of saline (Con24) or endotoxin (ET24) infusion.

\begin{tabular}{|c|c|c|c|c|c|c|c|c|c|}
\hline \multirow[b]{2}{*}{ Glucose } & \multicolumn{3}{|c|}{ Cono } & \multicolumn{3}{|c|}{ Con24 } & \multicolumn{3}{|c|}{ ET24 } \\
\hline & 4649 & \pm & 178 & 5218 & \pm & 237 & 4092 & \pm & $150^{\S}$ \\
\hline Lactate & 506 & \pm & 30 & 524 & \pm & $54^{\circ}$ & 796 & \pm & $62^{ \pm 8}$ \\
\hline Urea & 3535 & \pm & 451 & 1368 & \pm & $457^{\circ}$ & 2729 & \pm & $227^{\ddagger}$ \\
\hline Glutamate & 138 & \pm & 17 & 62 & \pm & $10^{\circ}$ & 60 & \pm & 3 \\
\hline Glutamine & 354 & \pm & 17 & 269 & \pm & $13^{\circ}$ & 210 & \pm & $20^{5}$ \\
\hline Glycine & 469 & \pm & 51 & 480 & \pm & 56 & 349 & \pm & $42^{\ddagger}$ \\
\hline Citrulline & 79 & \pm & 4 & 58 & \pm & $3^{\circ}$ & 42 & \pm & 4 \\
\hline Arginine & 92 & \pm & 4 & 106 & \pm & 4 & 70 & \pm & $3^{ \pm 5}$ \\
\hline Alanine & 185 & \pm & 12 & 118 & \pm & $14^{\circ}$ & 158 & \pm & $19^{\dagger}$ \\
\hline Tyrosine & 46 & \pm & 3 & 55 & \pm & 4 & 38 & \pm & $3^{+8}$ \\
\hline Valine & 352 & \pm & 18 & 347 & \pm & 29 & 294 & \pm & $26^{\dagger}$ \\
\hline Isoleucine & 138 & \pm & 8 & 158 & \pm & 21 & 114 & \pm & 10 \\
\hline Phenylalanine & 47 & \pm & 2 & 56 & \pm & $4^{\circ}$ & 88 & \pm & $6^{\ddagger 5}$ \\
\hline Leucine & 178 & \pm & 13 & 183 & \pm & 15 & 161 & \pm & 10 \\
\hline BCAA & 668 & \pm & 36 & 688 & \pm & 54 & 569 & \pm & $41^{\dagger}$ \\
\hline GNAA & 2354 & \pm & 78 & 2171 & \pm & 80 & 1804 & \pm & 113 \\
\hline $\mathrm{AA}$ & 2713 & \pm & 91 & 2470 & \pm & $95^{*}$ & 2096 & \pm & 141 \\
\hline
\end{tabular}

Data are mean \pm SEM in $\mu \mathrm{M}$. BCAA: branched-chain amino acids, GNAA: sum of measured gluconeogenic amino acids (in bold), AA: sum of measured amino acids. Essential amino acids in italics. Statistical analysis by Two-Way ANOVA: ${ }^{*} \mathrm{P}<0.05,{ }^{\circ} \mathrm{P}<0.01$; starvation plus infusion effect, ${ }^{\dagger} \mathrm{P}<0.05,{ }^{ \pm} \mathrm{P}<0.01$; endotoxin effect and ${ }^{8} \mathrm{P}<0.05,{ }^{\mathrm{S}} \mathrm{P}<0.01$; starvation $\mathrm{x}$ endotoxin effect. Complete list of amino acids available on request.

\section{Portal-Drained Viscera}

Fasting reduced the intestinal efflux of lactate from the portal-drained viscera (Table 4). Also, the total amino acid efflux and glutamine influx decreased. Phenylalanine isotope measurements showed that the net protein release across the portal-drained viscera diminished after fasting although neither protein synthesis, nor protein breakdown was significantly altered due to high variation in SA values (Figure $3 A$ ). Likewise, the efflux of valine from the portal-drained viscera decreased (Figure 3B). Based on the phenylalanine to valine molecular ratio in intestinal protein, the rate of valine transaminated and not used for protein synthesis did not significantly change (Con24 vs. Con0 group: 250 vs. $\left.140 \mathrm{nmol} \cdot \mathrm{kg} \mathrm{bw}^{-1} \cdot \mathrm{min}^{-1} ; \mathrm{P}>0.05\right)$. 

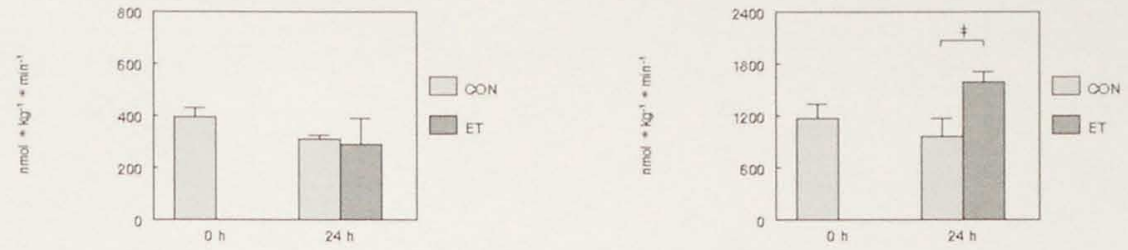

Production

Production
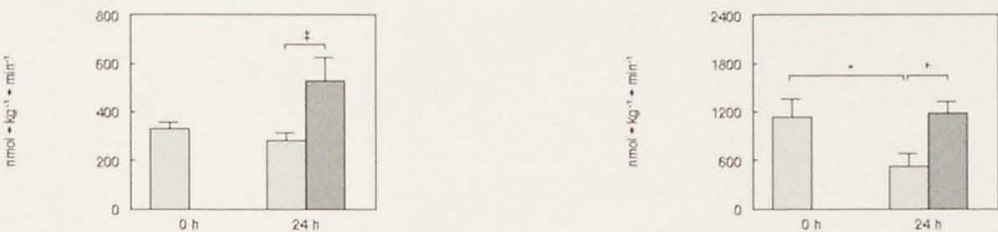

Net Balance

Net Balance
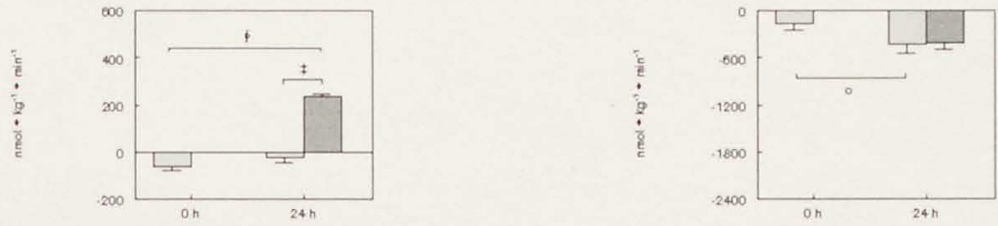

Figure 2. Disposal, production and net balance $(+=$ release $)$ of $A$ : phenylalanine and $B$ : valine across the hindquarter, before (Con0) and $24 \mathrm{~h}$ after the start of saline (Con24) or endotoxin (ET24) infusion. Statistical analysis by Two-Way ANOVA: ${ }^{\star} P<0.05,{ }^{\circ} P<0.01$; starvation plus infusion effect, ${ }^{\top} P<0.05,{ }^{\ddagger} P<0.01$; endotoxin effect and ${ }^{\S} \mathrm{P}<0.01$; starvation $x$ endotoxin effect.

\section{Liver}

Urea and glucose efflux from the liver decreased during fasting (Table 5). The contribution of the glucose efflux to the whole body glucose production reduced during fasting from $93 \%$ (Con0) to $44 \%$ (Con24). Concomitantly, glutamine and alanine influx decreased. The total amino acid output decreased to about zero. Decreased production of phenylalanine (Figure 4A) and valine (Figure 4B) was observed; the latter indicating that liver protein degradation was reduced. The estimated rate of phenylalanine used for hydroxylation, was lower during intermediate fasting in the Con 24 group $(140 \mathrm{nmol} \bullet \mathrm{kg}$ $\left.\mathrm{bw}^{-1} \cdot \mathrm{min}^{-1}\right)$ than during short-term fasting in the Con0 group $\left(270 \mathrm{nmol} \cdot \mathrm{kg} \mathrm{bw}^{-1} \cdot \mathrm{min}^{-1}\right.$, $P<0.05$ ). 

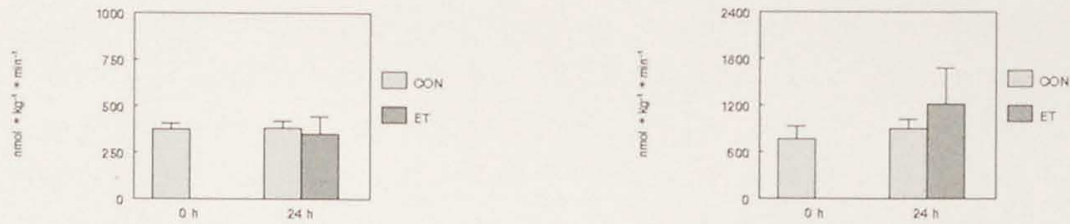

Production

Production
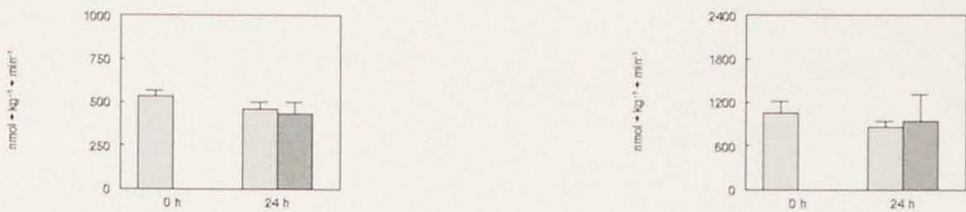

Net Balance

Net Baiance
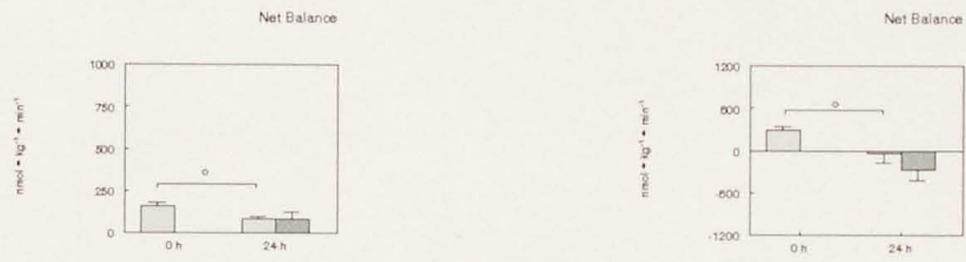

Figure 3. Disposal, production and net balance $(+=$ release) of $\mathrm{A}$ : phenylalanine and $\mathrm{B}$ : valine across the portal-drained viscera, before (Con0) and $24 \mathrm{~h}$ after the start of saline (Con24) or endotoxin (ET24) infusion. Statistical analysis by Two-Way ANOVA: ${ }^{\circ} \mathrm{P}<0.01$; starvation plus infusion effect, no endotoxin effect and no starvation $\times$ endotoxin effect was observed.

\section{Effect of 24-h endotoxin infusion}

A rise in body temperature (Table 1) was observed in endotoxin-treated as compared to control pigs. Both arterial hematocrit and $\mathrm{P}_{\mathrm{CO} 2}$ were lower in endotoxemic than in control pigs. Endotoxin increased the plasma flow to the portal-drained viscera and liver and tended to increase the flow to the hindquarter $(P=0.08)$. The mean arterial pressure transiently increased from $74 \pm 5$ at $0 \mathrm{~h}$ to $110 \pm 10 \mathrm{mmHg}$ at $4.5 \mathrm{~h}$, stabilizing again at $79 \pm 10 \mathrm{mmHg}$ at $8 \mathrm{~h}(80 \pm 10 \mathrm{mmHg}$ at $24 \mathrm{~h})$. The heart rate of the pigs increased from $88 \pm 20$ at $0 \mathrm{~h}$ to $188 \pm 15 \mathrm{bpm}$ (beats per $\mathrm{min}$ ) at $3.5 \mathrm{~h}$, gradually dropping to $120 \pm 20$ $\mathrm{bpm}$ at $24 \mathrm{~h}$. The endotoxin infusion decreased the arterial BCAA, glucose, glutamine, glycine, citrulline and arginine concentrations but increased lactate and phenylalanine concentrations (Table 2). The arterial concentration of the fibrinogen was not different between the two groups (ET24: $0.99 \pm 0.12$, Con24: $1.04 \pm 0.07 \mathrm{~g} / \mathrm{L}$ ). Neither was the haptoglobin concentration (ET24: $0.59 \pm 0.03$, Con24: $0.58 \pm 0.03 \mathrm{~g} / \mathrm{L}$ ). A lower total protein concentration was observed when pigs were challenged with endotoxin (ET24: $22.3 \pm 2.1$, Con24: $31.3 \pm 2.3 \mathrm{~g} / \mathrm{L}, \mathrm{P}=0.05)$. Endotoxin infusion increased the whole body appearance (Figure 1) of glucose, of phenylalanine (indicating protein breakdown) and valine. 


\section{Hindquarter}

During endotoxemia, the glucose influx into the hindquarter increased concomitant with lactate efflux from the hindquarter (Table 3). Furthermore, endotoxin infusion enhanced glutamate influx 1.2-fold, reversed total amino acids influx into efflux and increased the glutamine and alanine efflux about 3 and 6 times, respectively. The phenylalanine isotope measurements demonstrate that endotoxin accelerates protein breakdown but not the protein synthesis in the hindquarter (Figure 2A). The protein degradation in the hindquarter was twice as high in the endotoxin-treated compared to the control group. Assuming that the hindquarter muscle represents $50 \%$ of whole body muscle, the contribution of the total muscle protein breakdown to the whole body protein breakdown amounted to about $40 \%$ in the controls and $52 \%$ in the endotoxemic pigs. The increase in protein breakdown by the hindquarter accounted $43 \%$ for the increase in protein breakdown observed at whole body level. Endotoxin increased both valine disposal and production contributing $77 \%$ to the increased whole body valine turnover (Figure $2 \mathrm{~B}$ ). The rate of valine not used in protein synthesis, and therefore, metabolized by oxidation and transamination based on the valine to phenylalanine molecular ratio in muscle protein, was 2.5-fold higher in endotoxin-treated than in control pigs (620 vs. 250

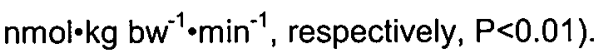

Table 3. Net balance $(+=$ efflux) across the hindquarter in the postabsorptive state before (Con0) and $24 \mathrm{~h}$ after the start of saline (Con24) or endotoxin (ET24) infusion.

\begin{tabular}{|c|c|c|c|c|c|c|c|c|c|}
\hline \multirow[b]{2}{*}{ Glucose } & \multicolumn{3}{|c|}{ Cono } & \multicolumn{3}{|c|}{ Con24 } & \multicolumn{3}{|c|}{ ET24 } \\
\hline & -4450 & \pm & 561 & -3670 & \pm & 932 & -7042 & \pm & $965^{\ddagger}$ \\
\hline Lactate & 3275 & \pm & 516 & 2438 & \pm & 685 & 4785 & \pm & $645^{\S}$ \\
\hline Urea & -1575 & \pm & 113 & -881 & \pm & $186^{\circ}$ & -1087 & \pm & 280 \\
\hline Glutamate & 1112 & \pm & 202 & 552 & \pm & $109^{*}$ & 1872 & \pm & $269^{\S}$ \\
\hline Glutamine & 365 & \pm & $566^{x}$ & -298 & \pm & $53^{x}$ & 1235 & \pm & 183 \\
\hline Glycine & -38 & \pm & $41^{ \pm}$ & -85 & \pm & $25^{*}$ & 80 & \pm & $19^{* \delta}$ \\
\hline Citrulline & -176 & \pm & 36 & -168 & \pm & $42^{\circ}$ & 205 & \pm & $40^{\S}$ \\
\hline Arginine & 500 & \pm & 122 & 398 & \pm & $87^{\circ}$ & 2515 & \pm & $639^{ \pm \S}$ \\
\hline Alanine & 8 & \pm & $35^{x}$ & -93 & \pm & $56^{x}$ & 259 & \pm & $58^{1 \S}$ \\
\hline Tyrosine & -160 & \pm & 81 & -429 & \pm & $115^{\circ}$ & -409 & \pm & $86^{\ddagger \S}$ \\
\hline Valine & -217 & \pm & 76 & -278 & \pm & $59^{\star}$ & 424 & \pm & $180^{\ddagger \S}$ \\
\hline Isoleucine & -64 & \pm & 16 & -24 & \pm & $23^{x_{0}}$ & 236 & \pm & $11^{ \pm \S}$ \\
\hline Phenylalanine & -323 & \pm & 45 & -381 & \pm & $90^{\circ}$ & 249 & \pm & $66^{\ddagger \S}$ \\
\hline$B C A A$ & -700 & \pm & $129^{x}$ & -1088 & \pm & $181^{\circ}$ & 264 & \pm & $192^{1 \S}$ \\
\hline GNAA & -905 & \pm & $545^{*}$ & -2092 & \pm & $679^{* 0}$ & 7113 & \pm & $1411^{\text {} \S}$ \\
\hline$A A$ & -1261 & \pm & $938^{*}$ & -2464 & \pm & $809^{x \star}$ & 8323 & \pm & $1516^{\ddagger 5}$ \\
\hline
\end{tabular}

Data are mean \pm SEM in nmol $\mathrm{kg} \mathrm{bw}^{-1} \cdot \mathrm{min}^{-1}$. BCAA: branched-chain amino acids, GNAA: sum of measured gluconeogenic amino acids (in bold), AA: sum of measured amino acids. Essential amino acids in italics. Statistical analysis by Two-Way ANOVA: ${ }^{*} P<0.05$, ${ }^{\circ} P<0.01$; starvation plus infusion effect, ${ }^{t} P<0.05$, ${ }^{\ddagger} P<0.01$; endotoxin effect and ${ }^{8} P<0.05,{ }^{\S} P<0.01$; starvation $x$ endotoxin effect. ${ }^{7} P>0.05$ : not significantly different from zero by Wilcoxon. Complete list of amino acids available on request. 


\section{Portal-Drained Viscera}

The lactate efflux from the portal-drained viscera increased during endotoxemia when compared to controls (Table 4) while glucose influx almost significantly increased $(P=0.06)$. Also, a remarkable glycine and tyrosine efflux from the portal-drained viscera was induced by endotoxin. In addition, endotoxin reduced glutamine influx by $40-50 \%$, alanine efflux by $50-60 \%$, arginine efflux by $63 \%$. Both an increase in glutamate and BCAA influx into the portal-drained viscera were observed. The rate of protein synthesis and breakdown in the portal-drained viscera, as measured from phenylalanine isotope (Figure $3 \mathrm{~A}$ ), was not affected by endotoxin challenge. Also, valine disposal and production remained unchanged (Figure $3 \mathrm{~B}$ ) whereas the estimated rate of valine degradation (transamination and oxidation) was higher in the ET24 compared to Con24 group (620 vs. $250 \mathrm{nmol} \cdot \mathrm{kg} \mathrm{bw}^{-1} \cdot \mathrm{min}^{-1}, \mathrm{P}<0.05$ ).

A Phenylalanine

Disposa

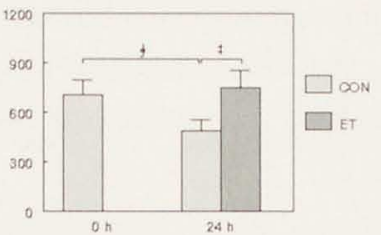

Production

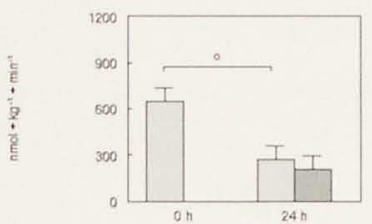

Net Balance

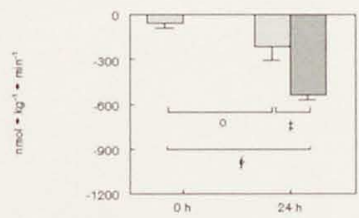

B. Valine

Disposal

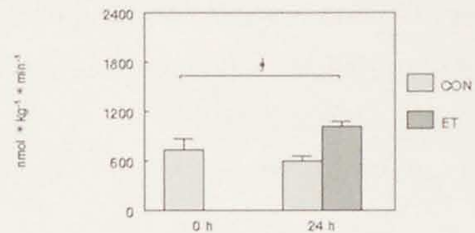

Production

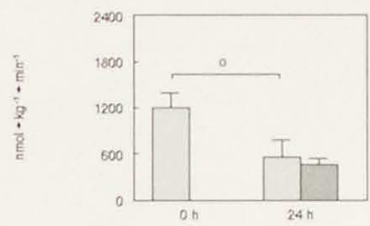

Not Balance

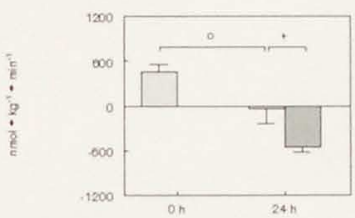

Figure 4. Disposal, production and net balance (+= release) of $\mathrm{A}$ : phenylalanine and $\mathrm{B}$ : valine across the liver, before (Con0) and $24 \mathrm{~h}$ after the start of saline (Con24) or endotoxin (ET24) infusion. Statistical analysis by Two-Way ANOVA: ${ }^{\circ} \mathrm{P}<0.01$; starvation plus infusion effect, ${ }^{\dagger} \mathrm{P}<0.05,{ }^{\ddagger} \mathrm{P}<0.01$; endotoxin effect and ${ }^{\S} \mathrm{P}<0.01$; starvation $x$ endotoxin effect. 


\section{Liver}

In the liver, endotoxin-induced increase in lactate influx was accompanied by increased glucose efflux. The rate of lactate uptake to glucose release was 1.6 in endotoxemic compared to 1.3 in control pigs. The contribution of glucose produced by the liver (data not shown) to glucose produced at whole body level was higher in the endotoxemic than in the control group ( $83 \%$ vs. $44 \%$ ). In addition to lactate, amino acid influx into the liver was higher during endotoxin treatment (Table 5). The net uptake of glycine was highest, followed by alanine and glutamine. The total amino acid influx matched the total amino acid efflux from muscle (estimated from $2 \times$ hindquarter) and portal-drained viscera together. Only $60 \%$ of the net glutamate efflux from the liver was taken up by muscie and portal-drained viscera tissues. The hepatic urea efflux substantially increased by endotoxin infusion but did not reach significance due to high variation in test results. Endotoxin administration increased both phenylalanine (Figure 4A) and valine disposal (Figure 4B) by the liver, the latter implying enhanced hepatic protein synthesis. The rate of phenylalanine disposal not used for protein synthesis did not significantly change (592 $\mathrm{nmol} \cdot \mathrm{kg} \mathrm{bw}^{-1} \cdot \mathrm{min}^{-1}$ in ET24 vs. 423 in Con24 group, $\mathrm{P}>0.05$, respectively).

Table 4. Net balance $(+=$ efflux) across the portal-drained viscera (zintestine) in the postabsorptive state before (Con 0 ) and $24 \mathrm{~h}$ after the start of saline (Con24) or endotoxin (ET24) infusion.

\begin{tabular}{lrrrr}
\hline & \multicolumn{2}{c}{ Cono } & \multicolumn{1}{c}{ Con24 } & \multicolumn{1}{c}{ ET24 } \\
\hline Glucose & $-4051 \pm 574$ & $-4319 \pm 1176$ & $-6170 \pm 1151$ \\
Lactate & $3088 \pm 460$ & $1919 \pm 769^{*}$ & $4979 \pm 944^{8}$ \\
Urea & $-800 \pm 948^{*}$ & $588 \pm 269^{*}$ & $-561 \pm 882^{*}$ \\
Glutamate & $-211 \pm 65$ & $-121 \pm 45$ & $-344 \pm 105^{\dagger}$ \\
Glutamine & $-1213 \pm 118$ & $-691 \pm 35^{\circ}$ & $-374 \pm 176^{8}$ \\
Glycine & $1593 \pm 497$ & $-37 \pm 216^{*}$ & $1043 \pm 383^{\dagger}$ \\
Citrulline & $486 \pm 34$ & $286 \pm 25^{\circ}$ & $345 \pm 60$ \\
Arginine & $324 \pm 56$ & $239 \pm 76$ & $65 \pm 17^{\dagger}$ \\
Alanine & $958 \pm 100$ & $436 \pm 55^{\circ}$ & $186 \pm 110^{*}$ \\
Tyrosine & $153 \pm 29$ & $31 \pm 20^{*}$ & $348 \pm 87^{\dagger 5}$ \\
Valine & $288 \pm 53$ & $-39 \pm 136^{* 0}$ & $-276 \pm 151^{*}$ \\
Isoleucine & $218 \pm 52$ & $159 \pm 36^{*}$ & $40 \pm 18^{\dagger}$ \\
Phenylalanine & $155 \pm 20$ & $79 \pm 16^{\circ}$ & $80 \pm 40^{*}$ \\
Leucine & $257 \pm 72$ & $201 \pm 32$ & $61 \pm 6^{\star}$ \\
BCAA & $763 \pm 145$ & $321 \pm 86^{\circ}$ & $-175 \pm 88^{* \dagger}$ \\
GNAA & $3082 \pm 615$ & $505 \pm 224^{\circ}$ & $1684 \pm 549^{\ddagger}$ \\
AA & $3866 \pm 718$ & $764 \pm 336^{*}$ & $2173 \pm 722^{\dagger}$ \\
\hline
\end{tabular}

Data are mean \pm SEM in nmol $\cdot \mathrm{kg} \mathrm{bw}^{-1} \cdot \mathrm{min}^{-1}$. BCAA: branched-chain amino acids, GNAA: sum of measured gluconeogenic amino acids (in bold), AA: sum of measured total amino acids. Essential amino acids in italics. Statistical analysis by Two-Way ANOVA: ${ }^{*} P<0.05,{ }^{\circ} \mathrm{P}<0.01$; starvation plus infusion effect, ${ }^{\dagger} \mathrm{P}<0.05$, ${ }^{7} \mathrm{P}<0.01$; endotoxin effect and ${ }^{8} \mathrm{P}<0.05,{ }^{5} \mathrm{P}<0.01$; starvation $\times$ endotoxin effect. ${ }^{\mathrm{P}} \mathrm{P}>0.05$ : not significantly different from zero by Wilcoxon. Complete list of amino acids available on request. 
Table 5. Net balance $(+=$ efflux) across the liver in the postabsorptive state before (Con0) and $24 \mathrm{~h}$ after the start of saline (Con24) or endotoxin (ET24) infusion.

\begin{tabular}{|c|c|c|c|c|c|}
\hline \multirow[b]{2}{*}{ Glucose } & \multicolumn{2}{|c|}{ Cono } & Con24 & \multicolumn{2}{|c|}{ ET24 } \\
\hline & 17375 & \pm 1359 & $9058 \pm 1834$ & 18833 & $\pm 2030^{\ddagger}$ \\
\hline Lactate & -12912 & \pm 883 & $-14658 \pm 1859^{\circ}$ & -25677 & $\pm 1768^{ \pm 5}$ \\
\hline Urea & 3260 & \pm 440 & $2420 \pm 455^{*}$ & 7360 & \pm 1683 \\
\hline Glutamate & 5838 & \pm 460 & $3202 \pm 444^{\circ}$ & 4461 & \pm 741 \\
\hline Glutamine & -1154 & \pm 220 & $-42 \pm 215^{x}$ & -1538 & $\pm 369^{ \pm 8}$ \\
\hline Glycine & -798 & $\pm 468^{x}$ & $-481 \pm 692^{x}$ & -4143 & $\pm 665^{\S}$ \\
\hline Citrulline & 15 & $\pm 49^{x}$ & $-13 \pm 31^{*}$ & -10 & $\pm 81^{*}$ \\
\hline Arginine & -138 & \pm 71 & $-327 \pm 44^{\circ}$ & -487 & $\pm 79^{8}$ \\
\hline Alanine & -2568 & \pm 221 & $-1743 \pm 188$ & -4010 & $\pm 526^{\dagger \S}$ \\
\hline Tyrosine & -149 & $\pm 36^{*}$ & $-216 \pm 71^{\times 0}$ & -610 & $\pm 145^{ \pm 5}$ \\
\hline Valine & 835 & \pm 205 & $-41 \pm 204^{* 0}$ & -672 & $\pm 145^{\dagger}$ \\
\hline Isoleucine & 435 & \pm 84 & $-79 \pm 133^{\times 0}$ & -291 & \pm 35 \\
\hline Phenylalanine & -56 & $\pm 35^{x}$ & $-217 \pm 90^{\circ}$ & -541 & $\pm 27^{\ddagger \S}$ \\
\hline Leucine & 551 & \pm 114 & $50 \pm 96^{x_{0}}$ & -513 & $\pm 81^{\dagger}$ \\
\hline $\mathrm{BCAA}$ & 1821 & \pm 280 & $-70 \pm 389^{* 0}$ & -1476 & $\pm 223^{\ddagger}$ \\
\hline GNAA & 2818 & \pm 701 & $-421 \pm 1298^{* 0}$ & -11815 & $\pm 1154^{\ddagger 5}$ \\
\hline AA & 3427 & \pm 863 & $-241 \pm 1357^{x_{0}}$ & -12249 & $\pm 1272^{\ddagger \S}$ \\
\hline
\end{tabular}

Data are mean \pm SEM in nmol $\cdot \mathrm{kg} \mathrm{bw}^{-1} \cdot \mathrm{min}^{-1}$. BCAA: branched-chain amino acids, GNAA: sum of measured gluconeogenic amino acids (in bold), AA: sum of measured total amino acids. Essential amino acids in italics. Statistical analysis by Two-Way ANOVA: ${ }^{*} P<0.05,{ }^{\circ} \mathrm{P}<0.01$; starvation plus infusion effect, ${ }^{\dagger} \mathrm{P}<0.05$, ${ }^{7} P<0.01$; endotoxin effect and ${ }^{8} P<0.05,{ }^{5} P<0.01$; starvation $x$ endotoxin effect. ${ }^{*} P>0.05$ : not significantly different from zero by Wilcoxon. Complete list of amino acids available on request.

\section{Discussion}

In the present model of hyperdynamic sepsis, the effect of 24-h endotoxin challenge on organ protein metabolism and inter-organ amino acid fluxes was investigated. The uses of catheter cannulation and isotope techniques enabled simultaneous measurements of protein synthesis and degradation across the portal-drained viscera, liver and hindquarter region and their contributions to whole body changes. In view of the likelihood that (semi)starvation accompanies the endotoxemia related metabolic response, a mock-treated control group allowed differentiation between effects evoked by starvation and by endotoxemia. The hemodynamic response of the pigs to the endotoxin infusion, which included elevated heart rate and preserved blood flow to the organs, represents the development of a circulatory state characteristic of hyperdynamic, but also hypermetabolic sepsis.

\section{Effect fasting}

During starvation of intermediate duration, glucose appears not to be the principal fuel for muscle since in this state of starvation glucose influx and lactate efflux across the 
hindquarter do not appear to change significantly. Enhanced uptake of total amino acids, especially the gluconeogenic and branched-chain amino acids, implies either their increased utilization or their decreased production from protein degradation. Phenylalanine isotope measurements revealed that muscle protein breakdown decreased as result of the fasting period. Decreased muscle protein degradation is also supported by the observation that production of valine, which is not de novo synthesized and can only derive from protein breakdown, decreased. Reduced glutamine and alanine release from the hindquarter would limit the nitrogen disposal during starvation of longer duration. A period of low muscle protein turnover prior to onset of accelerated protein breakdown is consistent with findings in starved rats $(4,8,9,19)$.

The glucose uptake across the portal-drained viscera did not change during starvation whereas the efflux of its metabolic product lactate decreased (30). This suggests that glucose-derived pyruvate is directed towards oxidation rather than towards lactate production. This is supported by the finding that also glutamate influx and alanine efflux decreased in this stage of starvation. Moreover, glutamine to citrulline flux seems reduced since glutamine influx and citrulline efflux decrease. This is in agreement with findings in rats (9) and rabbits (25) that were subjected to 3 days of starvation. The efflux of the sum of amino acids from the portal-drained viscera was reduced during starvation, which may be related to the decreased visceral protein breakdown.

Reduced amino flux from both hindquarter and portal-drained viscera areas contribute to a decreased amino acid supply to the liver. Despite the decline in liver glucose release, whole body glucose appearance did not diminish during starvation so that the relative contribution of glucose production by the liver becomes less. It is indeed known that during long-term starvation, gluconeogenesis is progressively taken over by the kidneys (7). Throughout the starvation period this allows the organism to keep plasma glucose concentration remarkably constant as demonstrated by unaltered whole body production and unaltered plasma levels of glucose. The reduced liver amino acid clearance may account for the decreased urea release. A similar reduction in liver urea synthesis was observed in rats starved for 1 day, prior to increased urea synthesis (9). Assuming that valine is not newly synthesized in the liver, the decreased valine disposal and production implies that not only gluconeogenesis, but also liver protein turnover is inhibited during starvation. Moreover, the substantial decrease in glutamate efflux and glutamine influx suggest that liver glutaminase activity is decreased during fasting. Accordingly, liver glutamine influx was found to progressively change to net release during starvation (46).

The rate of glucose entering the muscle and portal-drained viscera during starvation remains unchanged while the rate of glucose intermediary products leaving these organs 
decreased. Moreover, protein synthesis in muscle and portal-drained viscera is reduced. This implies that in these organs increased oxidation for energy production takes place.

In starved pigs, the glutamine released by the muscle matches the glutamine taken up by the portal-drained viscera and liver, which excludes a major role for the kidneys in glutamine uptake during starvation. Also, the rate of alanine released by muscle and portal-drained viscera matches liver alanine uptake. On the other hand, the uptake of glutamate by hindquarter muscle and portal-drained viscera accounted for only $60 \%$ of the glutamate released by the liver which means that the remaining glutamate is taken up by other organs. Although the organ uptake and release of these amino acids proportionally remained unchanged in the time course of starvation, the absolute flux rates decreased.

\section{Effect endotoxemia}

This model of mild endotoxemia shows that net protein catabolism of the hindquarter is induced by increased protein breakdown while no significant changes in protein synthesis could be determined. Although net muscle protein catabolism was described to result from both stimulation of proteolysis and inhibition of protein synthesis $(3,35,40)$, inhibition of protein synthesis is only small in comparison with increased proteolysis (41, 43). The contribution of muscle protein breakdown was estimated to represent one third of the whole body protein breakdown and the increase in hindquarter protein degradation predominantly accounted for the increase at whole body level. This emphasizes that changes in protein metabolism by this organ largely determine whole body kinetics. Differences in glucose and lactate fluxes are schematically illustrated in Figure 5A and B.
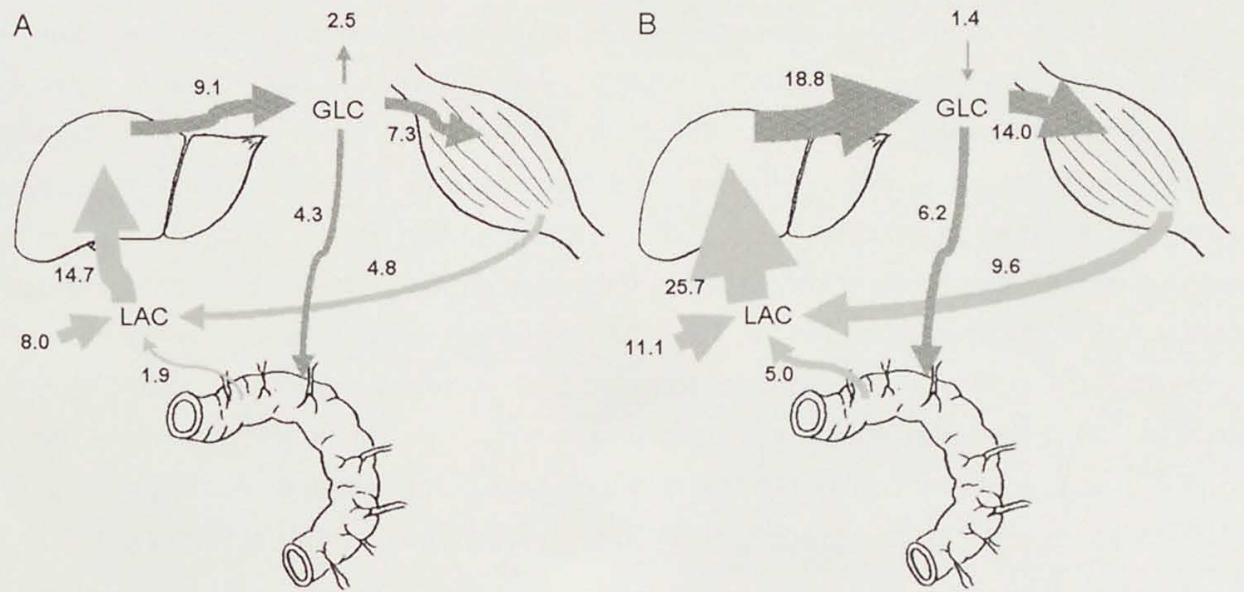

Figure 5. Fluxes in $\mu \mathrm{mol} \cdot \mathrm{kg} \mathrm{bw}^{-1} \cdot \mathrm{min}^{-1}$ of glucose and lactate across the muscle (estimated from $2 \times$ hindquarter), portal-drained viscera and liver during starvation A: without (Con24) and $\mathrm{B}$ : with 24-h endotoxin intervenion (ET24). The thickness of the arrow proportionally represents the rate of substrate flux. 
The observation that across the hindquarter, net glucose influx and lactate and alanine efflux simultaneously increased during endotoxemia, indicates that pyruvate oxidation in muscle is limited, which has previously been reported in endotoxemic rats (20). Inhibited pyruvate oxidation results in increased lactate, but also alanine production for which glutamate is required. Accordingly, lactate and alanine effluxes from the hindquarter increased in the endotoxin challenged pigs. BCAA's derived from accelerated protein degradation $(17,22)$ are preferentially subjected to transamination, their amino-group being transferred to $\alpha$-ketoglutarate to meet the increased requirements for glutamate (16). Their carbon chain formed in this process is, under conditions of a relative lack of energy such as sepsis (33), used for oxidation in the TCA cycle, providing energy for muscle cells. Increased giutamate formation not only serves alanine synthesis, but increased glutamine synthetase-mediated glutamine synthesis as well. Significant rises in glutamate influx and glutamine and alanine efflux from the hindquarter in amounts greater than would be anticipated from breakdown of muscle protein suggests that both alanine and glutamine de novo synthesis are elevated in muscle during endotoxemia. The observed increased valine disposal by the hindquarter suggests that intramuscular $B C A A$ transamination and oxidation is enhanced while the observed increased valine production reflects the release of BCAA's from increased protein breakdown that are not disposed and released into the circulation.

The increase in glucose influx and lactate efflux across the portal-drained reflects elevated lactate formation via pyruvate. In the gut, liver-derived glucose is an important substrate of lactate (15). Endotoxin-stimulated absorptive cells of the gut (18), lymphocytes of gut-associated lymphoid tissue and the spleen participate to large extent in this increased glucose utilization (20). Protein synthesis and degradation of the portaldrained viscera remained unaffected during endotoxemia. This contrasts with studies in rats given a single endotoxin dose that showed increased gut protein synthesis under endotoxemic conditions (50). Differences in endotoxemia model may underlie the discrepancy in observations made. The reduced glutamine uptake as observed in this model of endotoxemia is conform reduced intestinal glutamine uptake in septic rats (1) and patients (32). Initially, decreased glutaminase expression was assumed to underlie reduced glutamine uptake (32). However, increased glutamine synthetase expression was also found present in the gut under conditions of endotoxemia (26). If the latter holds true, enhanced BCAA and glutamate uptake by the portal-drained viscera may reflect their increased disposal for increased endogenous glutamine synthesis. Moreover, since visceral citrulline efflux remained unchanged, proline instead of glutamine may serve as a source of citrulline (48). The use of a glutamine isotope could provide more insight in the underlying mechanism of reduced glutamine influx. In response to endotoxin, the portal-drained viscera released a major amount of glycine providing $35 \%$ of the 
gluconeogenic precursors for the liver. The high amounts of glycine released may originate from glycine-conjugated bile acids, reflecting the enteric-hepatic cycle (5).

Enhanced net uptake of gluconeogenic amino acids and lactate, and simultaneous glucose net release by the liver imply that the rate of gluconeogenesis is increased. While amino acid and lactate carbons serve as precursors for accelerated gluconeogenesis, the nitrogen moieties of the deaminated amino acids are detoxified resulting in the observed increased urea formation. The contribution of hepatic glucose production to whole body glucose production doubled, implying a decreased contribution of the kidney to whole body glucose production. During endotoxemia, the hepatic glucose release almost matched the glucose uptake by the portal-drained viscera and muscle (Figure 5B). Liver, however, consumed more lactate than released from the portal-drained viscera and muscle implying that other organs including erythrocytes are involved in surplus lactate production. Although we could not detect changes in concentrations of measured acute-phase proteins, the liver protein synthesis in this endotoxemia model was accelerated. Possibly the protein secretion from liver into plasma occurs in a later stage of the pigs acute-phase response. However, concentration levels due to variation with hydration state may not be an appropriate reflection of synthesis or production rates. The amino acids that are taken up by the liver, therefore, not only serve gluconeogenesis but protein synthesis as well. Under endotoxemic conditions, the synthesis of acute-phase reactive proteins in the liver $(6,42)$ is enhanced. The phenylalanine to tyrosine hydroxylation was not affected by endotoxemia, which is in agreement with other studies of infection (45).

A

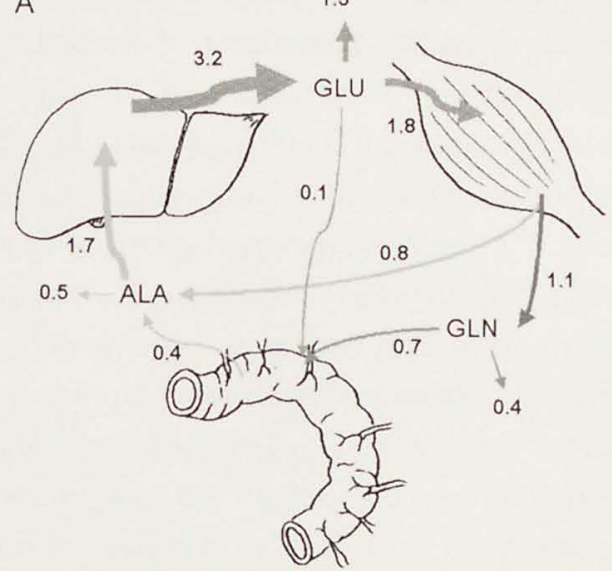

B

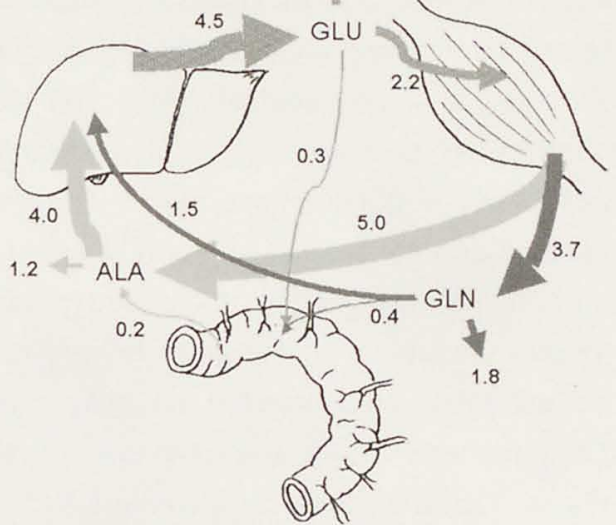

Figure 6 . Fluxes in $\mu \mathrm{mol} \cdot \mathrm{kg} \mathrm{bw}^{-1} \cdot \mathrm{min}^{-1}$ of alanine, glutamate and glutamine across the muscle (estimated from $2 \times$ hindquarter), portal-drained viscera and liver during starvation A: without (Con24) and B: with 24-h endotoxin intervenion (ET24). The thickness of the arrow proportionally represents the rate of substrate flux. 
The changes in glutamate, glutamine and alanine inter-organ fluxes in the state of fasting and of endotoxemia are illustrated schematically in Figure $6 \mathrm{~A}$ and $\mathrm{B}$, respectively. Only half of the glutamate release of hepatic origin is consumed by the muscle and gut, suggesting a major role for other organs such as nerve and cardiac tissue in glutamate utilization. Glutamate in muscle may serve as nitrogen intermediate for synthesis of alanine and glutamine, which are considered to play an important role in the muscle-liver amino acid exchange (29). During endotoxemia, the net output of alanine and glutamine by muscle exceeded the net consumption by the splanchnic region (i.e., liver plus portaldrained viscera). The remaining glutamine and alanine production by muscle may furnish fuel for endotoxin-activated immune and repair systems in other tissues.

In summary, the present study demonstrates that the metabolic response to long-term fasting includes reduction of the degradation of protein in all organs studied, i.e. the hindquarter muscle, liver and portal-drained viscera. Moreover, glutamine, lactate and alanine synthesis in muscle are limited. Inhibited amino acid effluxes from muscle and visceral organs accompany inhibited liver gluconeogenesis and ureagenesis. This normal protein-conserving mechanism is not observed following the onset of sepsis in which the metabolic response entails rapid breakdown of the muscle protein reserves, which is one of the main characteristics of sepsis. Endotoxemia accelerates hindquarter protein catabolism yielding amino acids for own use, but, predominantly, for protein gain and gluconeogenesis in the liver. Protein gain in the liver is effected by substantial increase in protein synthesis whereas protein loss in muscle is largely effected by an increase in protein degradation. The track-of is negative leading to protein loss at a whole body level because large part of the amino acids derived from muscle protein loss are used for gluconeogenesis and urea formation. Endotoxemia not only generates a substantial flux of gluconeogenic amino acids but also of glucose-derived lactate from the muscle to the liver. Besides the muscle, the portal-drained viscera appear to participate in furnishing gluconeogenic amino acids (12\% vs. $72 \%$ from muscle) and, more important, lactate $(60 \%$ vs. $30 \%$ from muscle). The liver becomes a main glutamate provider for hindquarter muscle, but also for other tissues. The protein turnover in the portal-drained viscera remained unaffected by hyperdynamic endotoxemia. The main changes in visceral substrate fluxes included substantial increase in lactate and glycine efflux and increased BCAA uptake. Changes in visceral substrate fluxes, however, contributed relatively little compared to hindquarter and liver.

Hindquarter- but also portal-drained viscera-derived glutamine, alanine and substantially glycine play a pivotal role in the substrate supply to the liver for enhanced ureagenesis, gluconeogenesis and protein synthesis during endotoxemia. 


\section{Acknowledgments}

The authors wish to express many thanks to Mr. H.M.H. van Eijk and Mr. J.L.J.M. Scheijen for analytical amino acid measurements, Mrs. K. Slot for help by acute-phase protein measurements and Mrs. G.A.M. ten Have for assistance during experimental procedures. This study was supported by grant 902-23-098 from The Netherlands Organization for Scientific Research.

\section{Reference}

1. Ardawi, M. S., Y. S. Jamal, A. A. Ashy, H. Nasr, and E. A. Newsholme. Glucose and glutamine metabolism in the small intestine of septic rats. J Lab Clin Med 115: 660-8, 1990.

2. Biolo, G., R. Y. D. Fleming, S. Maggi, and R. R. Wolfe. Transmembrane transport and intracellular kinetics of amino acids in human skeletal muscle. Am J Physiol 268: E75-E84, 1995.

3. Breuille, D., M. Arnal, F. Rambourdin, G. Bayle, D. Levieux, and C. Obled. Sustained modifications of protein metabolism in various tissues in a rat model of long-lasting sepsis. Clin Sci (Colch) 94: 413-23, 1998.

4. Cherel, Y., D. Attaix, D. Rosolowska-Huszcz, R. Belkhou, J. P. Robin, M. Arnal, and Y. Le Maho. Whole-body and tissue protein synthesis during brief and prolonged fasting in the rat. Clin Sci 81: 611-9, 1991.

5. Choi, J., J. H. Klinkspoor, T. Yoshida, and S. P. Lee. Lipopolysaccharide from Escherichia coli stimulates mucin secretion by cultured dog gallbladder epithelial cells. Hepatology 29: 1352-7, 1999.

6. Clowes, G. H. A. Amino acid transfer between muscle and the visceral tissues in man during health and disease. In: Problems and Potential of Branched-Chain Amino Acids in Physiology and Medicine, edited by R. Odessey. Amsterdam: Elsevier Science publishers B.V., 1986 , p. 299-334.

7. Corssmit, E. P., J. A. Romijn, and H. P. Sauerwein. Review article: Regulation of glucose production with special attention to nonclassical regulatory mechanisms: a review. Metabolism 50: 742-55., 2001.

8. De Blaauw, I., N. E. P. Deutz, and M. F. Von Meyenfeldt. Muscle protein and amino acid turnover in rats in vivo: effects of short-term and prolonged starvation. Clin Sci 90: 457-466, 1996.

9. Dejong, C. H. C., N. E. P. Deutz, and P. B. Soeters. Inter-organ nitrogen exchange during prolonged starvation in the rat. $J$ Clin Nutr Gastroenterol 6: 176-183, 1991.
10. Deutz, N. E. P., M. J. Bruins, and P. 8. Soeters. Infusion of soy and casein protein meals affect interorgan amino acid metabolism and urea kinetics differently in pigs. $J$ Nutr 128: 2435-2445, 1998.

11. Deutz, N. E. P., P. L. M. Reijven, G. Athanasas, and P. B. Soeters. Post-operative changes in hepatic, intestinal, splenic and muscle fluxes of amino acids and ammonia in pigs. Clin Sci 83: 607-614, 1992.

12. Deutz, N. E. P., P. L. M. Reijven, M. C. F. Bost, C. L. H. van Berlo, and P. B. Soeters. Modification of the effects of blood on amino acid metabolism by intravenous isoleucine. Gastroenterology 101: 1613-1620, 1991.

13. Deutz, N. E. P., A. J. M. Wagenmakers, and P. B. Soeters. Discrepancy between muscle and whole body protein turnover. Curr Opin Clin Nutr Metab Care 2: 29-32, 1999.

14. Fink, M. P., and S. O. Heard. Laboratory models of sepsis and septic shock. $J$ Surg Res 49: 186-196, 1990.

15. Fuller, M. F., and P. J. Reeds. Nitrogen cycling in the gut. Annu Rev Nutr 18: 385-411, 1998.

16. Goldberg, A. L., and T. W. Chang. Regulation and significance of amino acid metabolism in skeletal muscle. Fed Proc 37: 2301-7, 1978.

17. Groves, A. C., L. I. Woolf, J. H. Duff, and R. J. Finley. Metabolism of branched-chain amino acids in dogs with Escherichia coli endotoxin shock. Surgery 93: 273-278, 1983.

18. Lang, C. H., J. C. A. Obih, G. J. Bagby, J. N. Bagwell, and J. J. Spitzer. Endotoxin-induced increases in regional glucose utilization by small intestine: a TNF-independent effect. Am $J$ Physiol (Gastrintest Liver Physiol) 260(23): G548-G555, 1991.

19. Lowell, B. B., N. B. Ruderman, and M. N. Goodman. Regulation of myofibrillar protein degradation in rat skeletal muscle during brief and prolonged starvation. Metabolism 35: 1121-7, 1986. 
20. Meszaros, K., C. H. Lang, G. J. Bagby, and J. J. Spitzer. In vivo glucose utilization by individual tissues during nonlethal hypermetabolic sepsis. Faseb $J$ 2: 3083-6, 1988.

21. Michie, H. R. Metabolism of sepsis and multiple organ failure. World J Surg 20: 460-4. 1996.

22. Nawabi, M. D., K. P. Block, M. C. Chakrabarti, and $M . G$. Buse. Administration of endotoxin, tumor necrosis factor, or interleukin 1 to rats activates skeletal muscle branched-chain alpha-keto acid dehydrogenase. J Clin Invest 85: 256-63, 1990.

23. Newsholme, E. A., and A. R. Leech. Biochemistry for the Medical Sciences. New York: John Wiley \& Sons, 1983. p. 1-952.

24. Ooiwa, T., H. Goto, Y. Tsukamoto, T. Hayakawa, S. Sugiyama, N. Fujitsuka, and $Y$. Shimomura. Regulation of valine catabolism in canine tissues: tissue distributions of branched-chain aminotransferase and 2-oxo acid dehydrogenase complex, methacrylylCoA hydratase and 3-hydroxyisobutyryl-COA hydrolase. Biochim Biophys Acta 1243: 216220, 1995.

25. Sarac, T. P., W. W. Souba, J. H. Miller, C. K. Ryan, M. Koch, P. Q. Bessey, and H. C. Sax. Starvation induces differential small bowel luminal amino acid transport. Surgery 116: 679-85; discussion 685-6, 1994.

26. Sarantos, P., A. Abouhamze, R. Chakrabarti, and W. W. Souba. Glucocorticoids regulate intestinal glutamine synthetase gene expression in endotoxemia. Arch Surg 129: 5965., 1994.

27. Seglen, P. O., and A. E. Solheim. Effects of aminooxyacetate, alanine and other amino acids on protein synthesis in isolated rat hepatocytes. Biochim Biophys Acta 520: 63041, 1978.

28. Shinnick, F. L., and A. E. Harper. Branchedchain amino acid oxidation by isolated rat tissue preparations. Biochim Biophys Acta 437: 477-486, 1976.

29. Soeters, P. B., I. De Blaauw, B. A. C. Van Acker, M. F. Von Meyenfeldt, and N. E. P. Deutz. In vivo inter-organ protein metabolism of the splanchnic region and muscle during trauma, cancer and enteral nutrition. In: Bailliere's Clinical Endocrinology and metabolism, edited by B. Tindall Bailliere Tindall, 1997.

30. Soeters, P. B., and N. E. P. Deutz. Glutamine. Voeding 53: 266-268, 1992.
31. Soeters, P. B., and N. E. P. Deutz. Interorgan exchange of amino acids after trauma. In: Organ metabolism and nutrition: Ideas for future critical care, edited by T. H. Kinney JM. New York: Raven Press Ltd, 1994.

32. Souba, W. W., K. Herskowitz, V. S. Klimberg, R. M. Salloum, D. A. Plumley, T. C. Flynn, and E. M. Copeland, 3rd. The effects of sepsis and endotoxemia on gut glutamine metabolism. Ann Surg 211: 543-9; discussion 549-51., 1990.

33. Spydevold, O., and B. Hokland. Oxidation of branched-chain amino acids in skeletal muscle and liver of rat. Effects of octanoate and energy state. Biochim Biophys Acta 676: 27988., 1981.

34. Streat, S. J., and G. L. Hill. Nutritional support in the management of critically ill patients in surgical intensive care. World J Surg 11: 194201., 1987.

35. Svanberg, E., R. A. Frost, C. H. Lang, J. Isgaard, L. S. Jefferson, S. R. Kimball, and T. C. Vary. IGF-I/IGFBP-3 binary complex modulates sepsis-induced inhibition of protein synthesis in skeletal muscle. Am J Physiol Endocrinol Metab 279: E1145-58., 2000.

36. Ten Have, G. A. M., M. C. F. Bost, J. C. A. W. Suyk-Wierts, A. E. J. M. van den Bogaart, and N. E. P. Deutz. Simultaneous measurement of metabolic flux in portally-drained viscera, liver, spleen, kidney and hindquarter in the conscious pig. Lab Anim 30: 347-358, 1996.

37. Tourian, A., J. Goddard, and T. T. Puck. Phenylalanine hydroxylase activity in mammalian cells. J Cell Physiol 73: 159-170, 1969.

38. Van Eijk, H. M. H., D. R. Rooyakkers, and N. E. P. Deutz. Automated determination of polyamines by high-performance liquid chromatography with simple sample preparation. $J$ Chromatogr 730: 115-120. 1996.

39. Van Eijk, H. M. H., D. R. Rooyakkers, and N. E. P. Deutz. Rapid routine determination of amino acids in plasma by high-performance liquid chromatography with a 2-3 $\mu \mathrm{M}$ Spherisorb ODS II column. J Chromatogr 620: 143-148, 1993.

40. Vary, T. C. Regulation of skeletal muscle protein turnover during sepsis. Curr Opin Clin Nutr Metab Care 1: 217-24., 1998.

41. Vary, T. C., D. Dardevet, J. Grizard, L. Voisin, C. Buffiere, P. Denis, D. Breuille, and C. Obled. Differential regulation of skeletal muscle protein turnover by insulin and IGF-I after bacteremia. Am J Physiol 275: E584-93, 1998. 
42. Von Allmen, D., P. O. Hasselgren, T. Higashiguchi, and J. E. Fischer. Individual regulation of different hepatocellular functions during sepsis. Metabolism 41: 961-965, 1992.

43. von Allmen, D., P. O. Hasselgren, $T$. Higashiguchi, J. Frederick, O. Zamir, and J. E. Fischer. Increased intestinal protein synthesis during sepsis and following the administration of tumour necrosis factor alpha or interleukin-1 alpha. Biochem J 286: 585-9, 1992.

44. Wajngot, A., V. Chandramouli, W. C. Schumann, K. Kumaran, S. Efendic, and B. R. Landau. Testing of the assumptions made in estimating the extent of futile cycling. $A m ~ J$ Physiol 256: E668-75, 1989.

45. Wannemacher, R. W., Jr., A. S. Klainer, R. E. Dinterman, and W. R. Beisel. The significance and mechanism of an increased serum phenylalanine- tyrosine ratio during infection. Am J Clin Nutr 29: 997-1006, 1976.
46. Watford, M. Glutamine and glutamate metabolism across the liver sinusoid. $J$ Nutr 130: 983S-7S., 2000.

47. Wolfe, R. R. Radioactive and stable isotope tracers in biomedicine. Principles and practice of kinetic analysis. New York: Wiley-Liss, 1992.

48. Wu, G. Intestinal mucosal amino acid catabolism. J Nutr 128: 1249-1252, 1998.

49. Wu, G., T. L. Ott, D. A. Knabe, and F. W. Bazer. Amino acid composition of the fetal pig. J Nutr 129: 1031-8, 1999.

50. Zamir, O., P. O. Hasselgren, T. Higashiguchi, J. A. Frederick, and J. E. Fischer. Effect of sepsis or cytokine administration on release of gut peptides. Am J Surg 163: 181-185, 1992. 
Maaike J Bruins

Peter B Soeters

Nicolaas EP Deutz

Department of Surgery, Maastricht University, The Netherlands 


\section{Abstract}

The metabolic response following sepsis is characterized by net protein loss. Nutritional intervention often is applied to sustain whole body protein mass under such circumstances. The manner in which protein metabolism of the different organs is affected under nutrition-supported and post-septic circumstances remains ambiguous. Therefore, we explored the changes in in vivo organ and whole-body protein turnover following endotoxin-induced sepsis during enteral nutrition in the pig. The use of isotopes enabled simultaneous measurements of protein synthesis, breakdown and amino acid degradation across the portal-drained viscera (PDV, =intestinal tract), liver and hindquarter $(\approx 50 \%$ skeletal muscle). All pigs received continuous enteral infusion of a liquid meal equivalent to $0.3 \mathrm{~g}$ protein $\cdot \mathrm{kg}$ body weight $(\mathrm{bw})^{-1} \cdot \mathrm{h}^{-1} 3$ days prior to and 4 days after a 24-h endotoxemia period. Measurements were performed 1 day before and 1 and 4 days after endotoxemia that was induced by a $24-\mathrm{h}$ endotoxin $\left(3 \mu \mathrm{g} \cdot \mathrm{kg} \mathrm{bw}^{-1} \cdot \mathrm{h}^{-1}\right.$ LPS, $n=7)$ infusion. Controls received $\mathrm{NaCl}(n=7)$. Four days after endotoxemia, hindquarter protein turnover was increased resulting in net synthesis. The amino acid output by the PDV was increased 1 and 4 days post-endotoxemia. In the liver, net protein synthesis was enhanced 1 day after endotoxemia. Increased amino acid transamination in hindquarter and PDV led to glutamine and alanine effluxes that serve as substrate for liver and, possibly, immune system. By providing substrate, enteral nutrition can sustain elevated amino acid demand in the post-endotoxemic state by hindquarter, PDV and liver for protein synthesis and transamination processes.

\section{Introduction}

\section{Protein and amino acid metabolism during enteral nutrition}

One of the characteristic features of the post-septic state is a negative nitrogen balance resulting from net protein catabolism persisting for several days (4). In clinical practice, enteral nutritional support is often applied as a therapeutic intervention to restore protein and energy losses in situations where protein catabolism is manifest. The pro's and contra's of early feeding after sepsis have extensively been discussed. Most studies demonstrate the benefit of enteral nutrition as therapy after trauma by reducing complications and improving clinical outcome $(6,7,23)$. Nutritional intervention may be a preventive therapeutic measure to maintain gut integrity and in limiting the loss of protein from lean body mass by sustaining substrate-dependent protein synthesis $(5,22,23)$. Furthermore, adequate supply of amino acids in the early phase after sepsis may be of great benefit for visceral organs, specifically the liver, with respect to their increased 
substrate demands. Firstly, this may be of importance by providing amino acids for the process of hepatic gluconeogenesis, secondly, by supporting organ energy requirements and thirdly, by supporting visceral immune response and protein synthesis, i.e. of acute phase proteins $(4,8,9)$.

The loss of capacity to produce adequate amounts of endogenous immunonutrients, such as arginine and glutamine is one of the main characteristics in the inadequate immune response of septic patients. These amino acids may become "conditional" when the capacity to produce them is exceeded by their need. Nutritional supplementation of glutamine and arginine, often in combination with nucleotides and n-3 fatty acids, may overcome their depletion in stress situations and has been suggested to improve clinical outcome in critically ill patients.

\section{Protein studies}

Studies on whole body protein metabolism in several metabolic conditions like starvation, feeding, after trauma and during sepsis have been undertaken $(1,21)$. However, data from these studies are very difficult to interpret since they represent the sum of the turnover of several proteins in different organs that respond in different ways. Whole body protein studies provide insufficient insight in the relative importance of the different organs and their participation in protein metabolism. It seems preferable to assess the role that individual organs play in protein kinetics. In this study we used in vivo techniques to obtain information regarding protein and amino acid metabolism across the most important organs. Simultaneous measurements across the hindquarter $(\approx 50 \%$ skeletal muscle), the PDV (zintestinal tract) and the liver allow us, by the use of isotopetechniques, to understand the long-term effects of a sepsis period on the process of protein synthesis and degradation in these organs and their amino acid interactions during feeding. In the clinical situation, sepsis often is succeeded by early enteral nutritional support since early provision of enteral nutrients appears beneficial by reducing septic complications. Knowledge concerning the aspects of protein metabolism following endotoxemia during nutritional intervention is scarce and may be useful to devise nutritional support strategies in disease states associated with sepsis or inflammation.

\section{Choice of the sepsis model}

The aim of this model was to investigate the immediate (d 1) and delayed (d 4) effects of 24-h endotoxin intervention during nutritional support on glucose and protein kinetics in a setting that mimics a condition of post-septicemia. Bacterial endotoxins are often used in experimental models to study the mechanisms involved in inflammation and sepsis. Large doses of endotoxin lead to a hypodynamic profile whereas very small doses of endotoxin elicit a hypermetabolic response representative for hypermetabolic human 
sepsis (16). Therefore, pigs received small doses of endotoxin over an extended period. Pigs were supported by a fluid infusion to compensate for hypovolemia in order to evoke a hemodynamic profile in which cardiac output and organ perfusion are well preserved. For these reasons, we chose to induce an experimental sub-acute hypermetabolic model of sepsis. To reproduce the profile of a chronic human septic condition, endotoxin challenge was followed by enteral infusion of a liquid protein-enriched meal. The pig was chosen as chronically instrumented large animal model, because organ physiology and metabolic changes in this animal are in many respects comparable with those observed in humans $(15,26)$.

\section{Materials and methods}

\section{Animals}

Fourteen female Yorkshire $x$ Dutch Landrace crossbred pigs of 3 month old $(20-22 \mathrm{~kg}$ body weight (bw)) were individualiy housed. Pigs consumed water ad libitum and $1 \mathrm{~kg}$ of regular pig feed (Landbouwbelang, Roermond, the Netherlands; $16 \%$ crude protein (for composition see: (13)) daily in the morning, supporting a growth rate of $-300 \mathrm{~g} / \mathrm{d}$. The Animal Ethics Committee of the Maastricht University approved the study.

\section{Surgical Procedure}

After overnight food deprivation, all pigs were anesthetized with a mixture of $\mathrm{N}_{2} \mathrm{O} / \mathrm{O}_{2}$ (1:2) and halothane $(0.8 \%)$ and intubated. As antimicrobial prophylaxis, the pigs received 6.25 $\mathrm{mg} / \mathrm{kg}$ bw lincomycin and $12.5 \mathrm{mg} / \mathrm{kg}$ bw spectinomycin intravenously. Flunixine, a prostaglandin inhibitor, was administered intravenously for postoperative analgesia and to avoid activation of the coagulation system. A midline laparotomy was performed, and catheters were implanted using a method reported previously $(12,14)$. In brief, eight catheters (I.D. $1.0 \mathrm{~mm}$, O.D. $1.8 \mathrm{~mm}$, Tygon, Westvaco, Cleveland, $\mathrm{OH}$ ) were inserted in the abdominal aorta; one at a level just above the bifurcation (A1) and one at a level above the right renal vein (A2), two in a similar way in the inferior caval vein (V1 and V2 respectively) and in the portal $(P)$, hepatic $(H)$ and splenic vein $(S)$. The $A 1$ and the $S$ catheters were used for the purpose of para-aminohippuric acid $(\mathrm{PAH})$ infusion, the V2 catheter for isotope and endotoxin infusion and the A2, P, H and V1 catheters for blood sampling. Also a gastrostomy catheter (I.D. $1.6 \mathrm{~mm}$, O.D. $4.8 \mathrm{~mm}$, Tygon) was inserted. All catheters were tunneled through the abdominal wall and skin. To avoid catheter tip infections and to maintain patency, catheters were filled with a solution containing a mixture of gentamycin $(20 \mathrm{mg} / \mathrm{mL}$, Gentamycin $5 \%$, A.U.V., Cuyk, the Netherlands) and chymotrypsin (25 U/mL, Merck, Darmstadt, Germany) in $150 \mathrm{mmol} / \mathrm{L} \mathrm{NaCl}$ (saline). 


\section{Postoperative care}

Postoperative care was standardized (32). A canvas harness was fitted to each pig to protect the catheters and to allow easy handling of the animal. From the third day onwards, the pigs were fed $1 \mathrm{~kg}$ of food daily. During the whole recovery and experimental period (twenty days), the animals remained healthy without signs of infection. Four wk after the operation, the position of the catheter tips were checked under anesthesia using fluoroscopy. Subsequently, the pig was killed by an intravenous overdose of a barbiturate (Euthanasate $\odot$, Apharmo, Arnhem, the Netherlands).

\section{Experimental protocol}

Ten days after surgery, to standardize daily food intake, a liquid diet was enterally infused into the gastrostomy catheter via a swivel system connected to a pump. The liquid diet was prepared by mixing $4 \mathrm{~L}$ of Nutrison Steriflo High-Protein ${ }^{1}$ (osmolarity 255 mOsmol/L, Nutricia, Zoetermeer, the Netherlands) and $1 \mathrm{~L}$ of tap water. This enteral nutrition is a high-protein liquid meal composed for clinical patients with an optimal protein/energy ratio and containing all vitamins, minerals and other nutritional elements that meet the requirements of growing pigs (30). For that reason, no dietary supplements were added. Nutrition was given over 4 days (Figure 1). The first $d$, diet was infused at a rate of $2.5 \mathrm{~mL} \cdot \mathrm{kg}^{-1} \cdot \mathrm{h}^{-1}$ and the following three $\mathrm{d}$, pigs received $5 \mathrm{~mL}$ liquid diet $\cdot \mathrm{kg} \mathrm{bw}^{-1} \cdot \mathrm{h}^{-1}$

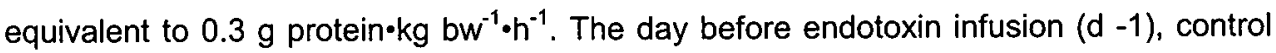
samples of the fed state were taken from 14 pigs (CON -1 group). The night before the endotoxin infusion (12 PM), enteral nutrition was stopped. The next morning ( $d 0,8$ AM), ), seven pigs received $3 \mu \mathrm{g} \cdot \mathrm{kg} \mathrm{bw}^{-1} \cdot \mathrm{h}^{-1}$ endotoxin (ET; lipopolysaccharide from Escherichia coli 055:B5, Sigma Chemicals Co., St. Louis, MO) in sterile pyrogen-free saline via the $\mathrm{V} 2$ catheter for a period of $24 \mathrm{~h}$. Seven control (CON) pigs received saline at an equivalent infusion rate. All pigs received saline as fluid support: $30 \mathrm{~mL} \cdot \mathrm{kg} \mathrm{bw}^{-1} \cdot \mathrm{h}^{-1}$ during the first $8 \mathrm{~h}$ and $20 \mathrm{~mL} \cdot \mathrm{kg} \mathrm{bw}^{-1} \cdot \mathrm{h}^{-1}$ the following $16 \mathrm{~h}$ to ensure the development of a hypermetabolic septic condition. Six $h$ after stopping the ET infusion enteral nutrition was restarted at $5 \mathrm{~mL} \cdot \mathrm{kg} \mathrm{bw}^{-1} \cdot \mathrm{h}^{-1}$. One day after stopping the ET infusion blood samples were taken from the $A 2, P, H, V 1$ catheters from the control group (CON 1) and from the endotoxin-treated pigs (ET 1). Four days after the ET period, again blood samples were taken from both groups (CON 4 and ET 4).

1 Proprietary diet consists of the following (per $1 \mathrm{~L}$ food): $63 \mathrm{~g}$ casein protein, $141 \mathrm{~g}$ carbohydrates; $11 \mathrm{~g}$ sugars, $128 \mathrm{~g}$ polysaccharides, 2 other, $49 \mathrm{~g}$ fat; $4 \mathrm{~g}$ saturated, 30 mono-unsaturated, $15 \mathrm{~g}$ polyunsaturated, minerals; $\mathrm{Na}, \mathrm{K}, \mathrm{Cl}, \mathrm{Ca}, \mathrm{P}, \mathrm{Mg}$, trace elements; $\mathrm{Fe}, \mathrm{Zn}, \mathrm{Cu}, \mathrm{Mn}, \mathrm{F}, \mathrm{Mo}, \mathrm{Se}, \mathrm{Cr}, \mathrm{l}$, vitamins; $\mathrm{A}$, B-6, B-12, C, D, E, K, thiamin, riboflavin, niacin, pantothenic acid, folic acid, $200 \mathrm{mg}$ choline. 


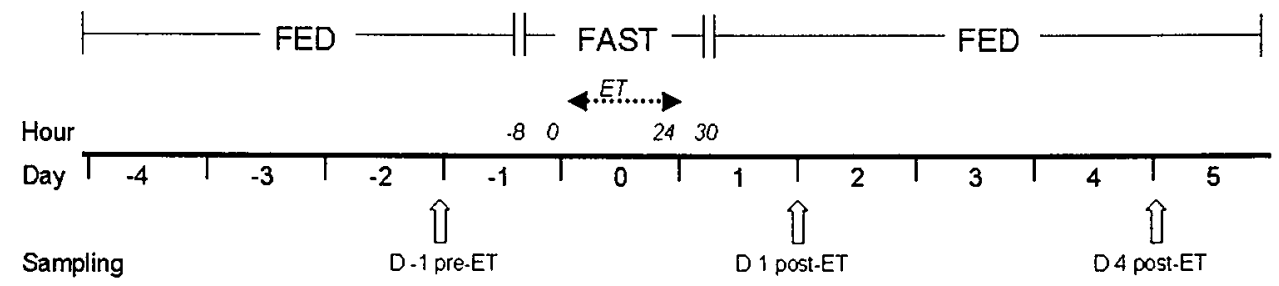

Figure 1: Illustration of the experimental design. ET: endotoxin.

\section{Infusion protocol}

On the morning of the trial, one $\mathrm{h}$ before start of the endotoxin infusion, a primed infusion of para-aminohippuric acid (PAH; 25 mmol/L, A 1422, Sigma Chemicals Co., St. Louis, $\mathrm{MO}$ ) was conducted. After an initial bolus of $5 \mathrm{~mL}$ PAH solution, an infusion was started at a rate of about $40 \mathrm{~mL} \cdot h^{-1}$ per catheter through the splenic vein and the aorta infusion catheters (32). Directly after the PAH bolus infusion, a priming dose $\left(1 \mu \mathrm{Ci} \cdot \mathrm{kg} \mathrm{bw}^{-1}\right)$ followed by a constant infusion $\left(1 \mu \mathrm{Ci} \cdot \mathrm{kg} \mathrm{bw}^{-1}\right)$ of $\mathrm{L}-\left[2,6{ }^{3} \mathrm{H}\right]$ phenylalanine and $\mathrm{L}-[3,4-$ ${ }^{3} \mathrm{H}$ ]valine (Amersham, Buckinghamshire, UK) and a primed $\left(0.5 \mu \mathrm{Ci} \cdot \mathrm{kg} \mathrm{bw}^{-1} \cdot \mathrm{h}^{-1}\right)$ constant infusion $\left(0.5 \mu \mathrm{Ci} \cdot \mathrm{kg} \mathrm{bw}^{-1} \cdot \mathrm{h}^{-1}\right)$ of $\mathrm{D}-\left[6-{ }^{3} \mathrm{H}\right] \mathrm{glucose}$ (NEN Dupont, NET-100A, Mechelen, Belgium) was given via the inferior caval vein catheter. Also via this catheter, a primed $\left(0.1 \mu \mathrm{mol} \cdot \mathrm{kg} \mathrm{bw}^{-1}\right)$ infusion $\left(0.1 \mu \mathrm{mol} \cdot \mathrm{kg} \mathrm{bw}^{-1} \cdot \mathrm{h}^{-1}\right)$ of L-[guanidino $-{ }^{15} \mathrm{~N}_{2}$ ]arginine (Mass Trace, Woburn, MA, USA) was started to calculate arginine kinetics and NO production. However, because of high enteral arginine infusion rates, NO production data were not reliable.

Before the start of the infusions, background blood samples were taken. One $\mathrm{h}$ after the start of the infusions, steady state conditions for PAH (data not shown), L-[2,6$\left.{ }^{3} \mathrm{H}\right]$ phenylalanine, L- $\left[3,4-{ }^{3} \mathrm{H}\right]$ valine, L-[guanidino- $-{ }^{15} \mathrm{~N}_{2}$ ]arginine and $\mathrm{D}-\left[6-{ }^{3} \mathrm{H}\right]$ glucose were obtained (34) and blood was sampled from catheters in the abdominal aorta, the portal, the hepatic and the caval vein.

\section{Sample processing}

Promptly after sampling, blood was distributed in pre-chilled, heparinized tubes (Sarstedt, Nümbrecht, Germany) on ice. Hematocrit was determined to enable calculation of plasma flow from blood flow. For blood gas analysis (arterial $\mathrm{pH}$, bicarbonate, $\mathrm{PO}_{2}$ and $\mathrm{PCO}_{2}$ ), $200 \mu \mathrm{L}$ blood was sealed airtight in heparinized $1 \mathrm{~mL}$ syringes and immediately analyzed on an automatic blood gas system (Acid Base Laboratory (ABL3), Radiometer, Copenhagen, Denmark). Temperature of the pig was measured rectally. Centrifugation was performed at $4{ }^{\circ} \mathrm{C}$ for 5 minutes at $8500 \mathrm{~g}$, plasma 
collected and kept on ice. For urea, glucose and lactate determinations $900 \mu \mathrm{L}$ plasma was added to $90 \mu \mathrm{L} 3 \mathrm{mmol} / \mathrm{L}$ trichloroacetic acid solution, ensuring stability of the substances. For amino acid analysis, $500 \mu \mathrm{L}$ of plasma was deproteinized by mixing with $20 \mathrm{mg}$ dry sulfosalicylic acid. For PAH determination, $300 \mu \mathrm{L}$ of blood was added to 600 $\mu \mathrm{L}$ of $0.7 \mathrm{mmol} / \mathrm{L}$ trichloroacetic acid solution, thoroughly mixed, centrifuged and supernatant collected. All samples were frozen in liquid nitrogen and stored at $-80^{\circ} \mathrm{C}$ until further analysis.

\section{Biochemical analysis}

$\mathrm{PAH}$ was detected spectrophotometrically after deacetylation of the supernatant at $100^{\circ} \mathrm{C}$ for $45 \mathrm{~min}(32)$ and plasma ammonia and urea were detected by standard enzymatic methods on an automated analysis system (Cobas Mira-S, Hoffmann-La Roche, Basel, Switzerland). Plasma amino acids were determined by a fully automated HPLC (Pharmacia, Woerden, the Netherlands) after precolumn derivatization with ophthaldialdehyde (35). Also, glucose and lactate concentrations were determined by HPLC. Glucose and amino acid fractions were collected and counted in respectively 6 and $20 \mathrm{~mL}$ Ultima Gold XR (Packard, Groningen, the Netherlands) for radioactivity on a liquid scintillation spectrophotometer to obtain their specific activity. Although phenylalanine can be converted into tyrosine and glucose into lactate, we could not detect sufficient radioactivity in their respective fractions to calculate a reliable specific activity. Enrichments of arginine were calculated as tracer to tracee ratios and were determined by a fully automated liquid chromatography-mass spectrometry (LC-MS, Thermoquest LCQ, Veenendaal, the Netherlands) system (34) that was, using column switching, in connection with the HPLC.

\section{Calculations}

Plasma flow ( $\mathrm{mL} \cdot \mathrm{kg} \mathrm{bw}^{-1} \cdot \mathrm{min}^{-1}$ ) was calculated using PAH as an indicator in an indicatordilution technique (32). Organ amino acid turnover was calculated in a two-compartment model (37). The substrate net balance was defined as NB (nmol•kg bw ${ }^{-1} \cdot \mathrm{min}^{-1}$ ) and calculated in (1) by multiplying the mean plasma flow by the difference between $\mathrm{V}$ ] and $[A]$ that represent the venous and arterial plasma concentration of the substrate $(\mu \mathrm{mol} / \mathrm{L})$, respectively. Therefore, a positive NB represents net release or efflux and a negative NB represents net uptake or influx of substrate across the organ. The tracer net balance across an organ ( $\mathrm{nb}, \mathrm{nmol} \cdot \mathrm{kg} \mathrm{bw}{ }^{-1} \cdot \mathrm{min}^{-1}$ ) was calculated in (2) in which $\mathrm{SA}_{A}$ and $S A_{V}$ represent the specific activities of the substrate in arterial and venous plasma, respectively.

(1) NB = flow PLASMA $\times([\mathrm{V}]-[\mathrm{A}])$

(2) $\mathrm{nb}=$ flow $\times\left\{\left([\mathrm{A}] \times S A_{A}\right)-\left([\mathrm{V}] \times S A_{V}\right)\right\}$ 
The disposal and production rate (nmoløkg bw ${ }^{-1} \cdot \mathrm{min}^{-1}$ ) across the PDV, the splanchnic region and the hindquarter were calculated in (3) and (4), respectively.

(3) $\quad$ Disposal $=\mathrm{nb} / \mathrm{SA}_{V}$

(4) Production $=$ Disposal + NB

For calculations of arginine kinetics, enrichments in stead of specific activities were used. The venous specific activity was used for the calculation of the disposal because it approaches best the intracellular specific activity (precursor pool) (3). Liver values were calculated by subtracting PDV from splanchnic values. In the PDV and hindquarter, the disposal of phenylalanine is a reflection of protein synthesis and production of phenylalanine of breakdown, as these organs have very low hydroxylase activity (33). Valine disposed in these organs can either become transaminated or be used in protein synthesis (29). In the liver, valine is mainly used for protein synthesis since its transamination rate is very low (29). Disposal of phenylalanine by the liver is a combination of protein synthesis and hydroxylation of phenylalanine to tyrosine (33). The rate of valine transamination in the PDV and hindquarter and the rate of phenylalanine hydroxylation in the liver were estimated from the assumption that the ratio of valine to phenylalanine in these organs constitutes 1.7 (40).

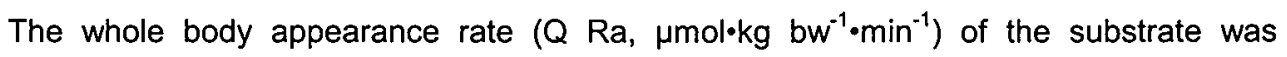
calculated as described before (37) and is shown in formula (5). I represents the rate of tracer infused ( $\mu$ moløkg bw ${ }^{-1} \cdot \mathrm{min}^{-1}$ ) and $\mathrm{Q}$ Ra represents the sum of endogenous appearance and appearance from enterally infused substrate absorbed by the gut.

(5) $Q R a=1 / S A_{A}$

Also across the PDV, the total production rate of a substrate measured is a combination of substrate derived from endogenous production plus output absorbed by the gut from the enterally infused meal that is not retained for disposal and escapes metabolism. This measured total production rate of a substrate $(P)$ by the PDV can be corrected for the contribution of substrate $\left(P_{N D}\right.$ ) that is derived from absorption of enteral infusion ( $E_{A B S}$ ) and is not metabolized or non-disposed. This gives a good approximate of the production of the substrate that represents endogenous production $\left(P_{E N D}\right)$ in the PDV (Figure 2$)$.

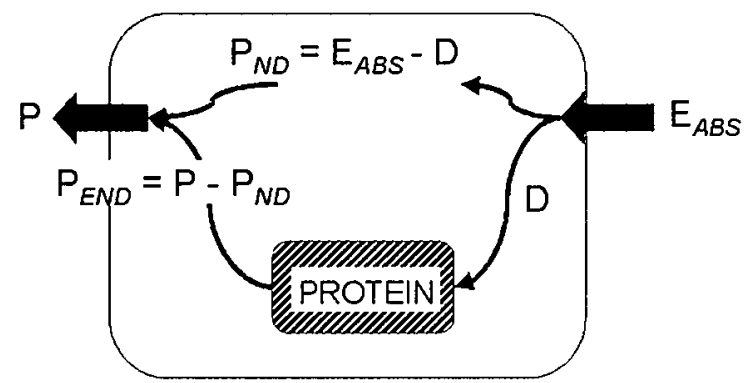

Figure 2: Calculation of correction of production of substrate for the contribution of substrate from enteral infusion that escapes disposal or metabolism $\left(\mathrm{P}_{\mathrm{ND}}\right)$. $\mathrm{P}_{\mathrm{ND}}$ is the difference between the rate of disposal (D) and substrate absorbed from enteral infusion $\left(E_{A B S}\right)$. The endogenous production $\left(P_{E N D}\right)$ of substrate by the portaldrained viscera is calculated by subtracting $P_{N D}$ from the total production $(P)$ as measured. 
Calculations of $E_{A B S}$ are based on the assumption that $90 \%$ of the enteral meal $(E)$ is absorpted, therefore, $E_{A B S}=0.9 \times E(13)$. $P_{N D}$ is the difference between $E_{A B S}$ and $D$, and $P_{E N D}$ can subsequently be calculated by subtracting $P_{N D}$ from the measured $P$.

Alpha-amino nitrogen ( $\alpha-A N)$ was calculated as the sum of the $\alpha$-amino acids. Branchedchain amino acids (BCAA) were calculated as the sum of valine, leucine and isoleucine. GNAA represented the sum of the gluconeogenic $\alpha$-amino acids (all amino acids occurring in protein except for leucine and lysine).

\section{Statistics}

Results are presented as means \pm SEM. Because very similar results were obtained at $d$ -1 in the CON and the ET group, pigs were pooled in one CON -1 group. The data were subjected to a 2-way analysis of variance (ANOVA) to compare group differences at $d 1$ and 4 post-ET, to test changes over time (d 1 and 4 post-ET versus $d-1$ pre-ET) within the CON group and interaction between time and group. Significance from zero was tested by the Wilcoxon's non-parametric test. Levels of significance were set at $P<0.05$.

\section{Results}

\section{Whole body variables}

The temperature and the arterial blood gas values did not change 1 or 4 days after the intervention of ET (Table 1). ET challenge significantly decreased the arterial hemoglobin $(\mathrm{Hb})$ at $\mathrm{d} 1$. The plasma flow of the portal drained viscera (PDV) did not significantly differ between the ET 1 and 4 and their CON groups. The hepatic plasma flow was higher in the ET 1 than in the CON 1 group. ET increased the hindquarter flow in a timedependent manner in the ET 1 and 4 groups.

\section{Arterial concentrations}

One day after ET challenge most amino acid concentrations were lower compared to the control group except for the glutamate concentration that was significantly higher in a time-dependent way (Table 2). Four days post-ET, the decreased plasma levels were maintained, except for glutamate, glutamine, glycine, alanine and lysine. One day after ET challenge, both arterial concentration of glucose and urea were lower in the ET 1 than in the CON 1 group.

\section{Whole body appearances (Q Ra)}

One day after ET intervention, no significant changes were observed in the $Q R a$ of phenylalanine (representing protein degradation), valine, arginine and glucose (Table 3). The Q Ra of arginine was higher in the ET 4 than in the CON 4 group. The Q Ra of valine decreased in time at $\mathrm{d} 4$ compared to $\mathrm{d}-1$. 
Table 1. Temperature, arterial blood gas values and plasma flow across the portal-drained viscera (PDV), liver and hindquarter in fed pigs, before $(d-1)$ and 1 and 4 days after 24-h saline (CON) or endotoxin (ET) infusion.

\begin{tabular}{|c|c|c|c|c|c|c|c|c|c|c|c|}
\hline & \multirow{2}{*}{$\frac{d-1}{\operatorname{CON}-1}$} & \multicolumn{2}{|c|}{ d 1} & \multicolumn{8}{|c|}{04} \\
\hline & & $\operatorname{CON} 1$ & ET 1 & \multicolumn{3}{|c|}{ ANOVA } & CON 4 & ET 4 & \multicolumn{3}{|c|}{ ANOVA } \\
\hline & \multicolumn{3}{|c|}{$n m o l \cdot k g ~ b w^{-1} \cdot \min ^{-1}$} & $P_{E T}$ & $\mathrm{PT}_{\mathrm{T}}$ & $P_{E T X T}$ & \multicolumn{2}{|c|}{$n m o l \cdot k g ~ b w^{-1} \cdot \min ^{-1}$} & $P_{E T}$ & $P_{T}$ & PETXT \\
\hline Temp, ${ }^{\circ} \mathrm{C}$ & $38.2 \pm 0.2$ & $38.0 \pm 0.2$ & $38.1 \pm 0.3$ & - & $\cdot$ & - & $37.5 \pm 0.4$ & $38.1 \pm 0.4$ & - & - & - \\
\hline $\mathrm{Hb}, \mathrm{mmol} / \mathrm{L}$ & $5.2 \pm 0.1$ & $4.9 \pm 0.1$ & $4.3 \pm 0.2$ & 0.05 & 0.01 & - & $4.8 \pm 0.2$ & $4.7 \pm 0.1$ & - & 0.01 & - \\
\hline $\mathrm{PH}$ & $7.44 \pm 0.01$ & $7.47 \pm 0.02$ & $7.47 \pm 0.01$ & - & 0.05 & - & $7.46 \pm 0.02$ & $7.46 \pm 0.01$ & - & 0.05 & - \\
\hline $\mathrm{PcO}_{\mathrm{co}} \mathrm{kPa}$ & $6.1 \pm 0.2$ & $6.1 \pm 0.2$ & $6.0 \pm 0.2$ & - & - & - & $5.6 \pm 0.2$ & $5.9 \pm 0.5$ & - & - & - \\
\hline $\mathrm{Po} 2, \mathrm{kPa}$ & $13.3 \pm 0.5$ & $12.3 \pm 1.0$ & $11.8 \pm 0.5$ & - & 0.05 & - & $11.8 \pm 0.6$ & $12.4 \pm 1.0$ & - & - & - \\
\hline $\mathrm{HCO}_{3}^{-}$ & $29.6 \pm 0.9$ & $31.0 \pm 1.7$ & $31.4 \pm 1.1$ & - & - & - & $29.7 \pm 1.2$ & $31.0 \pm 4.2$ & - & - & - \\
\hline \multirow[t]{2}{*}{ So2, \% } & $97.7 \pm 0.3$ & $97.8 \pm 0.4$ & $97.0 \pm 0.4$ & - & - & - & $97.6 \pm 0.4$ & $97.6 \pm 0.5$ & - & - & - \\
\hline & \multicolumn{3}{|c|}{$m L \cdot k g b w^{-1} \cdot \min ^{-1}$} & \multicolumn{8}{|c|}{$m L \cdot k g b w^{-1} \cdot \min ^{-1}$} \\
\hline Flow PDV & $36.2 \pm 1.3$ & $31.2 \quad 4.1$ & $42.0 \pm 7.0$ & - & - & - & $30.4 \pm 6.8$ & $32.4 \pm 2.9$ & - & - & - \\
\hline Flow liver & $49.8 \pm 2.9$ & $44.5 \pm 6.5$ & $56.8 \pm 5.7$ & 0.05 & - & - & $33.7 \pm 8.7$ & $44.2 \pm 6.7$ & - & - & - \\
\hline Flow & $30.4 \pm 3.0$ & $15.3 \pm 3.0$ & $36.4 \pm 5.6$ & - & - & 0.01 & $18.0 \pm 2.5$ & $31.7 \pm 2.5$ & - & - & 0.01 \\
\hline
\end{tabular}

Abbreviations used: $\mathrm{HCO}_{3}{ }^{-}$, bicarbonate; bw, body weight. Data are means \pm SEM. Number of pigs: CON group: $n=7$ and ET group: $n=7$. Statistics by 2 -way ANOVA: $P$ values of significance indicate probability of differences: $P_{E T}$, between $C O N$ and $E T$ at $d 1$ and $4 ; P_{T}$, within $C O N$ between $d 1$ or 4 and $d-1 ; P_{E T X T}$, no interaction between ET and time was observed.

Table 2. Arterial concentrations in fed pigs, before ( $d-1)$ and 1 and 4 days after the start of saline (CON) or endotoxin (ET) infusion.

\begin{tabular}{lccccccccccc}
\hline & $\mathrm{d}-1$ & \multicolumn{3}{c}{$\mathrm{d} 1$} & \multicolumn{3}{c}{$\mathrm{d} 4$} \\
\hline & $\mathrm{CON}-1$ & $\mathrm{CON} 1$ & $\mathrm{ET} 1$ & \multicolumn{1}{c}{ ANOVA } & \multicolumn{1}{c}{ CON 4} & ET 4 & ANOVA \\
\hline Glutamate & $142 \pm 29$ & $135 \pm 26$ & $218 \pm 28$ & 0.01 & - & - & $135 \pm 27$ & $184 \pm 29$ & - & - & - \\
Glutamine & $587 \pm 40$ & $723 \pm 60$ & $571 \pm 83$ & - & - & - & $575 \pm 49$ & $548 \pm 49$ & - & - & - \\
Glycine & $628 \pm 61$ & $618 \pm 95$ & $437 \pm 54$ & 0.05 & - & - & $593 \pm 65$ & $646 \pm 82$ & - & - & - \\
Citrulline & $106 \pm 7$ & $109 \pm 8$ & $74 \pm 7$ & 0.01 & - & - & $105 \pm 7$ & $93 \pm 7$ & 0.05 & - & - \\
Arginine & $130 \pm 11$ & $152 \pm 15$ & $88 \pm 9$ & 0.01 & - & - & $142 \pm 25$ & $89 \pm 7$ & 0.01 & - & - \\
Alanine & $369 \pm 37$ & $427 \pm 45$ & $317 \pm 35$ & 0.05 & - & - & $339 \pm 55$ & $371 \pm 48$ & - & - & - \\
Tyrosine & $140 \pm 11$ & $262 \pm 18$ & $129 \pm 22$ & 0.01 & 0.01 & - & $164 \pm 32$ & $121 \pm 14$ & 0.05 & - & - \\
Valine & $524 \pm 36$ & $517 \pm 39$ & $388 \pm 34$ & 0.01 & 0.05 & - & $469 \pm 25$ & $409 \pm 39$ & 0.010 .05 & - \\
Isoleucine & $181 \pm 15$ & $205 \pm 14$ & $150 \pm 18$ & 0.01 & - & - & $174 \pm 15$ & $168 \pm 15$ & 0.05 & - & - \\
Phenylalanin & $68 \pm 6$ & $114 \pm 10$ & $92 \pm 7$ & 0.01 & 0.01 & - & $78 \pm 10$ & $66 \pm 4$ & 0.05 & - & - \\
Ornithine & $164 \pm 12$ & $169 \pm 17$ & $96 \pm 15$ & 0.01 & 0.05 & - & $172 \pm 33$ & $105 \pm 10$ & 0.01 & - & - \\
Leucine & $244 \pm 24$ & $301 \pm 19$ & $217 \pm 20$ & 0.01 & - & - & $247 \pm 26$ & $215 \pm 21$ & 0.05 & - & - \\
Lysine & $225 \pm 43$ & $370 \pm 40$ & $226 \pm 30$ & 0.05 & - & - & $217 \pm 41$ & $193 \pm 20$ & - & - & - \\
BCAA & $949 \pm 72$ & $1023 \pm 62$ & $755 \pm 71$ & 0.01 & - & - & $890 \pm 62$ & $792 \pm 99$ & - & - & - \\
Q-AN & $4392 \pm 296$ & $5147 \pm 307$ & $3624 \pm 273$ & 0.01 & - & - & $4252 \pm 384$ & $3858 \pm 227$ & 0.05 & - & - \\
Glucose & $6307 \pm 173$ & $7318 \pm 356$ & $5939 \pm 309$ & 0.01 & - & - & $6158 \pm 312$ & $6499 \pm 340$ & - & - & - \\
Lactate & $671 \pm 46$ & $648 \pm 39$ & $733 \pm 68$ & 0.05 & - & - & $546 \pm 46$ & $667 \pm 57$ & 0.05 & - & - \\
Urea & $3674 \pm 316$ & $3317 \pm 486$ & $1987 \pm 240$ & 0.05 & - & - & $4859 \pm 101$ & $3885 \pm 770$ & - & - & - \\
\hline
\end{tabular}

Abbreviations used: BCAA, branched-chain amino acids; $\alpha$-AN, total amino nitrogen. Data are means \pm SEM. Number of pigs: CON group: $n=7$ and ET group: $n=7$. Statistics by 2-way ANOVA: $P$ values of significance indicate probability of differences: $P_{E T}$, between CON and ET at $d 1$ and $4 ; P_{T}$, within CON between $d 1$ or 4 and $d-1 ; P_{E T X T}$, no interaction between $E T$ and time was observed. 
Table 3. Whole body production of glucose, phenylalanine, valine and arginine in the fed state, before (day 1) and 1 and 4 days after the start of saline (CON) or endotoxin (ET) infusion.

\begin{tabular}{|c|c|c|c|c|c|c|c|c|c|c|c|}
\hline & \multirow{2}{*}{$\frac{d-1}{\operatorname{CON}-1}$} & \multicolumn{2}{|c|}{ d 1} & \multicolumn{8}{|c|}{ d 4} \\
\hline & & CON 1 & ET 1 & \multicolumn{3}{|c|}{ ANOVA } & $\operatorname{CON} 4$ & ET 4 & \multicolumn{3}{|c|}{ ANOVA } \\
\hline & \multicolumn{3}{|c|}{$n m o l \cdot k g ~ b w^{-1} \cdot \min ^{-1}$} & $P_{E T}$ & $\mathrm{P}_{\mathrm{T}}$ & PETXT & \multicolumn{2}{|c|}{$n m o l \cdot k g ~ b w^{-1} \cdot \min ^{-1}$} & $P_{E T}$ & $\mathrm{PT}_{\mathrm{T}}$ & PETXT \\
\hline Phenylalanine & $2.3 \pm 0.2$ & $2.3 \pm 0.3$ & $2.1 \pm 0.3$ & - & - & - & $2.0 \pm 0.1$ & $2.1 \pm 0.3$ & - & - & - \\
\hline Valine & $4.9 \pm 0.3$ & $4.3 \pm 0.4$ & $4.2 \pm 0.4$ & - & - & - & $4.0 \pm 0.2$ & $4.1 \pm 0.3$ & - & 0.01 & - \\
\hline Arginine & $1.9 \pm 0.2$ & $1.6 \pm 0.2$ & $1.5 \pm 0.2$ & - & 0.05 & - & $1.4 \pm 0.2$ & $1.8 \pm 0.2$ & 0.01 & - & - \\
\hline Glucose & $44.7 \pm 2.5$ & $49.5 \pm 4.4$ & $46.9 \pm 3.5$ & - & - & - & $43.1 \pm 6.2$ & $45.2 \pm 3.0$ & - & - & - \\
\hline
\end{tabular}

Abbreviations used: bw, body weight. Data are means \pm SEM. Data are not corrected for portal output from enteral infusion. Number of pigs: CON group: $n=7$ and ET group: $n=7$. Statistics by 2 -way ANOVA: $P$ values of significance indicate probability of differences: $P_{E T}$, between CON and ET at $d 1$ and $4 ; P_{T}$, within CON between $d 1$ or 4 and $d-1 ; P_{E T X T}$, no interaction between ET and time was observed.

\section{Hindquarter}

One and 4 days after ET infusion, the net uptake of glutamate and arginine and the efflux of glutamine was higher by the hindquarter by an interaction of ET and time. At d 4, the uptake of alanine and glycine was lower in the ET-treated pigs than in the controls (Table 4). The period of ET infusion had no significant effect on the glucose or lactate flux across the hindquarter.

\section{Phenylalanine, valine and arginine kinetics}

Compared to controls, the ET infusion had a time-related effect by increasing both the phenylalanine disposal (protein synthesis) and production (protein degradation) across the hindquarter at d 4 (Figure $3 \mathrm{~A}$ ). The net uptake of phenylalanine was higher in the ET 4 than in the CON 4 group. Both 1 and 4 days after ET challenge, valine disposal and production were also significantly higher compared to controls (Figure 3B). The estimated disposed valine used in the hindquarter transamination was significantly higher in the ET 1 than in the CON 1 (995 vs. $63 \mathrm{nmol} \cdot \mathrm{kg} \mathrm{bw}^{-1} \cdot \mathrm{min}^{-1}$ ). Similarly, the disposal ( $\mathrm{d}$ 1 and 4) and production (d 4) of arginine by the hindquarter were significantly higher in the ET-treated pigs than in their controls net resulting in higher influx of arginine (d 1 and 4 ) by the hindquarter (Figure $3 C$ ).
Table 6. Infusion rate of (secondary) amino acids, present in the enteral supplemented Nutrison-HP*.

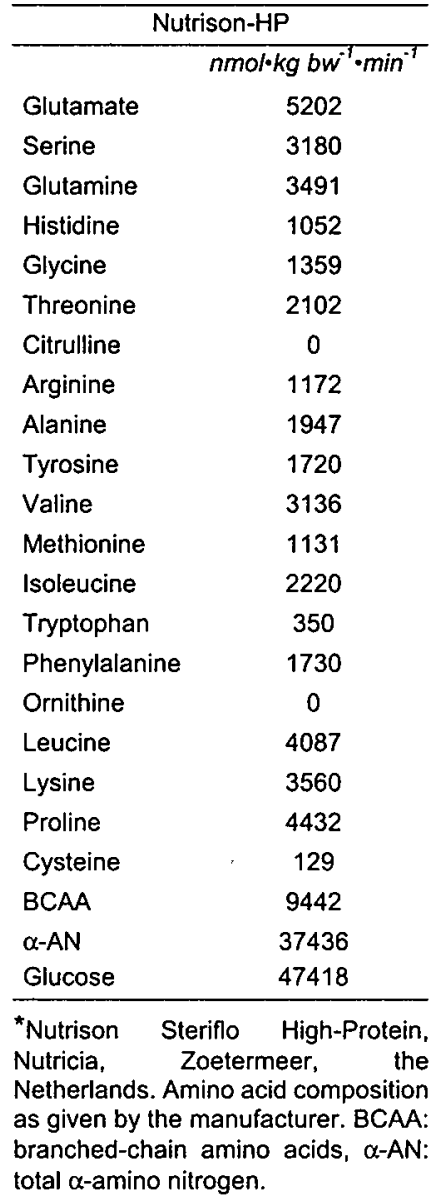


Table 4. Substrate net balances across the hindquarter in fed pigs, before ( $d-1)$ and 1 and 4 days after the start of saline (CON) or endotoxin (ET) infusion.

\begin{tabular}{|c|c|c|c|c|c|c|c|c|c|c|c|}
\hline & \multirow{2}{*}{$\frac{d-1}{\operatorname{CON}-1}$} & \multicolumn{2}{|c|}{ d 1} & \multicolumn{8}{|c|}{ d 4} \\
\hline & & CON 1 & ET 1 & & ANOV! & & $\mathrm{CON} 4$ & ET 4 & & NOV & \\
\hline & \multicolumn{3}{|c|}{$n \mathrm{~mol} \cdot k a b^{-1} \cdot \mathrm{min}^{-1}$} & $P_{E T}$ & $\operatorname{Pr}$ & $P_{E T \times T}$ & \multicolumn{2}{|c|}{$n m o l \cdot k g b w^{-9} \cdot \min ^{-1}$} & $P_{E T}$ & $P_{r}$ & $P_{E T X T}$ \\
\hline Glutamate & $-1812 \pm 426$ & $-779 \pm 324^{A}$ & $-2392 \pm 459$ & 0.05 & - & 0.05 & $-866 \pm 197$ & $-2180 \pm 182$ & 0.01 & - & 0.05 \\
\hline Glutamine & $1346 \pm 304$ & $469 \pm 308^{\#}$ & $1247 \pm 113$ & - & - & 0.05 & $547 \pm 137$ & $1371 \pm 239$ & - & - & 0.05 \\
\hline Glycine & $-2089 \pm \frac{1}{h}$ & $-547 \pm 185$ & $-629 \pm 175$ & - & - & - & $-922 \pm 215$ & $-533 \pm 264^{\#}$ & 0.05 & - & 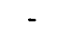 \\
\hline Arginine & $-653 \pm 94$ & $-419 \pm 51$ & $-822 \pm 159$ & - & 0.05 & 0.01 & $-373 \pm 123$ & $-610 \pm 65$ & - & - & 0.05 \\
\hline Alanine & $-1184 \pm 245$ & $-758 \pm 348^{\#}$ & $-453 \pm 259^{\#}$ & - & $\cdot$ & - & $-724 \pm 288$ & $-278 \pm 240^{\#}$ & 0.05 & 0.05 & \\
\hline Tyrosine & $-330 \pm 112$ & $-280 \pm 190^{*}$ & $-172 \pm 77^{*}$ & - & - & - & $-13 \pm 81^{z}$ & $-157 \pm 92^{\#}$ & - & - & - \\
\hline Valine & $23 \pm 174$ & $-585 \pm 80$ & $-754 \pm 224$ & - & 0.05 & - & $-614 \pm 151$ & $-972 \pm 248$ & - & - & - \\
\hline Isoleuci & $-919 \pm 194$ & $-534 \pm 72$ & $-438 \pm 113$ & - & 0.05 & - & $-343 \pm 104$ & $-653 \pm 74$ & - & - & - \\
\hline Phenylalanine & $-287 \pm 58$ & $-279 \pm 92$ & $-302 \pm 81$ & - & - & - & $-115 \pm 46$ & $-276 \pm 53$ & - & - & 0.05 \\
\hline Leucine & $-1221 \pm 248$ & $-899 \pm 170$ & $-751 \pm 209$ & - & - & - & $-605 \pm 120$ & $-921 \pm 183$ & - & - & - \\
\hline Lysine & $\begin{array}{r}-1238 \pm 757 \\
\mu \mathrm{n}\end{array}$ & $\begin{array}{l}-1193 \pm 530 \\
10 \mathrm{l} \cdot \mathrm{kg} \mathrm{bw}^{-1} \cdot \mathrm{mi}\end{array}$ & $\begin{array}{l}-1040 \pm 323 \\
n^{-1}\end{array}$ & - & - & - & $\begin{array}{r}-739 \pm 359 \\
\mu \mathrm{mol} / \mathrm{kg}\end{array}$ & $\begin{array}{c}-643 \pm 104 \\
b w^{-1} \cdot \min ^{-1}\end{array}$ & - & - & - \\
\hline$B C A A$ & $-3.4 \pm 0.7$ & $-1.9 \pm 0.2$ & $-1.9 \pm 0.5$ & - & 0.05 & - & $-1.5 \pm 0.3$ & $-2.5 \pm 1.1^{\#}$ & - & - & - \\
\hline$\alpha-A N$ & $-12.8 \pm 3.0$ & $-8.1 \pm 1.8$ & $-8.5 \pm 1.3$ & - & - & - & $-7.2 \pm 1$ & $-7.3 \pm 1.5$ & - & - & - \\
\hline Glucos & $-11.9 \pm 2.5$ & $-12.1 \pm 3.2$ & $-11.2 \pm 3.1$ & - & - & - & $-13.1 \pm 2.5$ & $-16.1 \pm 6.1^{*}$ & - & - & - \\
\hline Lactate & $-0.2 \pm 0.7^{\#}$ & $2.6 \pm 0.7$ & $1.0 \pm 1.1^{\#}$ & - & 0.05 & - & $0.7 \pm 0.6^{\#}$ & $0 \pm 1.4^{\#}$ & - & - & - \\
\hline
\end{tabular}

Abbreviations used: bw, body weight; BCAA, branched-chain amino acids; $\alpha$-AN, sum of amino acids measured. Data are means \pm SEM. $+=$ Net efflux or release. Number of pigs: CON group: $n=7$ and ET group: $n=7$. Statistics by 2 -way ANOVA: $P$ values of significance indicate probability of differences: $P_{E T}$. between CON and $E T$ at $d 1$ and $4 ; P_{T}$, within CON between $d 1$ or 4 and $d-1 ; P_{E T X T}$, interaction between ET and time. Wilcoxon test: "not different from zero.

Table 5. Substrate net balances across the portal-drained viscera in fed pigs, before (d -1) and 1 and 4 days after the start of saline (CON) or endotoxin (ET) infusion.

\begin{tabular}{|c|c|c|c|c|c|c|c|c|c|c|c|}
\hline & \multirow{2}{*}{$\frac{d-1}{\operatorname{CON}-1}$} & \multicolumn{2}{|c|}{ d 1} & \multicolumn{8}{|c|}{ d 4} \\
\hline & & CON 1 & ET 1 & \multirow{2}{*}{\multicolumn{5}{|c|}{$n m o l \cdot k g ~ b w^{-1} \cdot \mathrm{min}^{-1}$}} & \multicolumn{3}{|c|}{ ANOVA } \\
\hline & \multicolumn{3}{|c|}{$n m o l \cdot k g ~ b w^{-1} \cdot \mathrm{min}^{-1}$} & & & & & & $P_{E T}$ & $\mathrm{P}_{\mathrm{T}}$ & $P_{E T \times T}$ \\
\hline Glutamate & $-72 \pm 88^{\#}$ & $1250 \pm 1604^{\sharp}$ & $51 \pm 174^{\#}$ & - & - & 0.05 & $709 \pm 454^{\#}$ & $453 \pm 375^{\#}$ & - & - & - \\
\hline Glutamine & $-891 \pm 480$ & $-2281 \pm 903$ & $389 \pm 387^{\#}$ & - & - & 0.01 & $-1553 \pm 700$ & $274 \pm 393^{\#}$ & - & - & 0.05 \\
\hline Glycine & $1300 \pm 528^{\#}$ & $589 \pm 439^{*}$ & $1364 \pm 599^{\#}$ & - & - & - & $54 \pm 589^{\#}$ & $2010 \pm 679^{\#}$ & - & - & 0.05 \\
\hline Citrulline & $667 \pm 97$ & $413 \pm 78$ & $454 \pm 52$ & - & 0.05 & - & $607 \pm 95$ & $816 \pm 130$ & - & - & - \\
\hline Arginine & $75 \pm 121$ & $412 \pm 190$ & $857 \pm 136$ & 0.05 & - & 0.05 & $543 \pm 271$ & $970 \pm 122$ & 0.05 & - & - \\
\hline Alanine & $3477 \pm 563$ & $3066 \pm 367$ & $4297 \pm 419$ & 0.01 & - & - & $2493 \pm 854$ & $5742 \pm 138$ & 0.01 & - & - \\
\hline Tyrosine & $14 \pm 255$ & $452 \pm 100$ & $1230 \pm 345$ & - & - & - & $1225 \pm 279$ & $1319 \pm 273$ & - & 0.05 & - \\
\hline Valine & $907 \pm 261$ & $1075 \pm 266$ & $1808 \pm 398$ & 0.05 & - & - & $1440 \pm 388$ & $2153 \pm 145$ & 0.05 & 0.05 & - \\
\hline Isoleucine & $934 \pm 299$ & $842 \pm 221$ & $1395 \pm 213$ & 0.01 & - & - & $1340 \pm 146$ & $1887 \pm 393^{\#}$ & $\Rightarrow-$ & 0.01 & - \\
\hline Phenylalanine & $735 \pm 189$ & $772 \pm 118$ & $1000 \pm 79$ & 0.05 & - & - & $1007 \pm 251$ & $1233 \pm 132$ & - & - & 0.05 \\
\hline Ornithine & $214 \pm 123^{\#}$ & $449 \pm 418^{\#}$ & $675 \pm 198$ & - & - & - & $349 \pm 154$ & $832 \pm 265^{*}$ & - & - & - \\
\hline Leuci & $1578 \pm 446$ & $1775 \pm 342$ & $2606 \pm 491$ & 0.05 & - & - & $2122 \pm 313$ & $2712 \pm 274$ & 0.01 & - & - \\
\hline Lysine & $909 \pm 378^{\# \prime}$ & $840 \pm 242$ & $1509 \pm 228$ & 0.01 & - & - & $1447 \pm 244$ & $1839 \pm 196$ & - & 0.05 & 0.05 \\
\hline & & & & & & & & & & & \\
\hline BCAA & $3.7 \pm 0.7$ & $4.1 \pm 0.3$ & $6.0 \pm 0.5$ & 0.01 & - & - & $6.9 \pm 0.5$ & $7.1 \pm 1.6$ & 0.01 & - & - \\
\hline$\alpha-A N$ & $14.6 \pm 3.6$ & $13.8 \pm 1.6$ & $23.9 \pm 2.5$ & 0.01 & - & - & $17.5 \pm 3.4$ & $28.7 \pm 2.7$ & 0.01 & - & - \\
\hline Glucose & $25.7 \pm 5.0$ & $37.0 \pm 3.2$ & $39.0 \pm 4.7$ & - & - & - & $14.1 \pm 1.1$ & $19.5 \pm 1.9$ & - & - & - \\
\hline Lactate & $5.9 \pm 1.0$ & $7.3 \pm 1.1$ & $7.6 \pm 1.9$ & - & - & - & $3.3 \pm 1.3$ & $3.1 \pm 2.2^{\#}$ & - & - & - \\
\hline
\end{tabular}

Abbreviations used: bw, body weight; BCAA, branched-chain amino acids; $\alpha$-AN, sum of amino acids measured. Data are means \pm SEM. $+=$ Net efflux or release. Number of pigs: CON group: $n=7$ and ET group: $n=7$. Statistics by 2-way ANOVA: $P$ values of significance indicate probability of differences: $P_{E T}$, between $C O N$ and $E T$ at $d 1$ and $4 ; P_{T}$, within CON between $d 1$ or 4 and $d-1 ; P_{E T \times T}$, interaction between ET and time. Wilcoxon test: "not different from zero. 

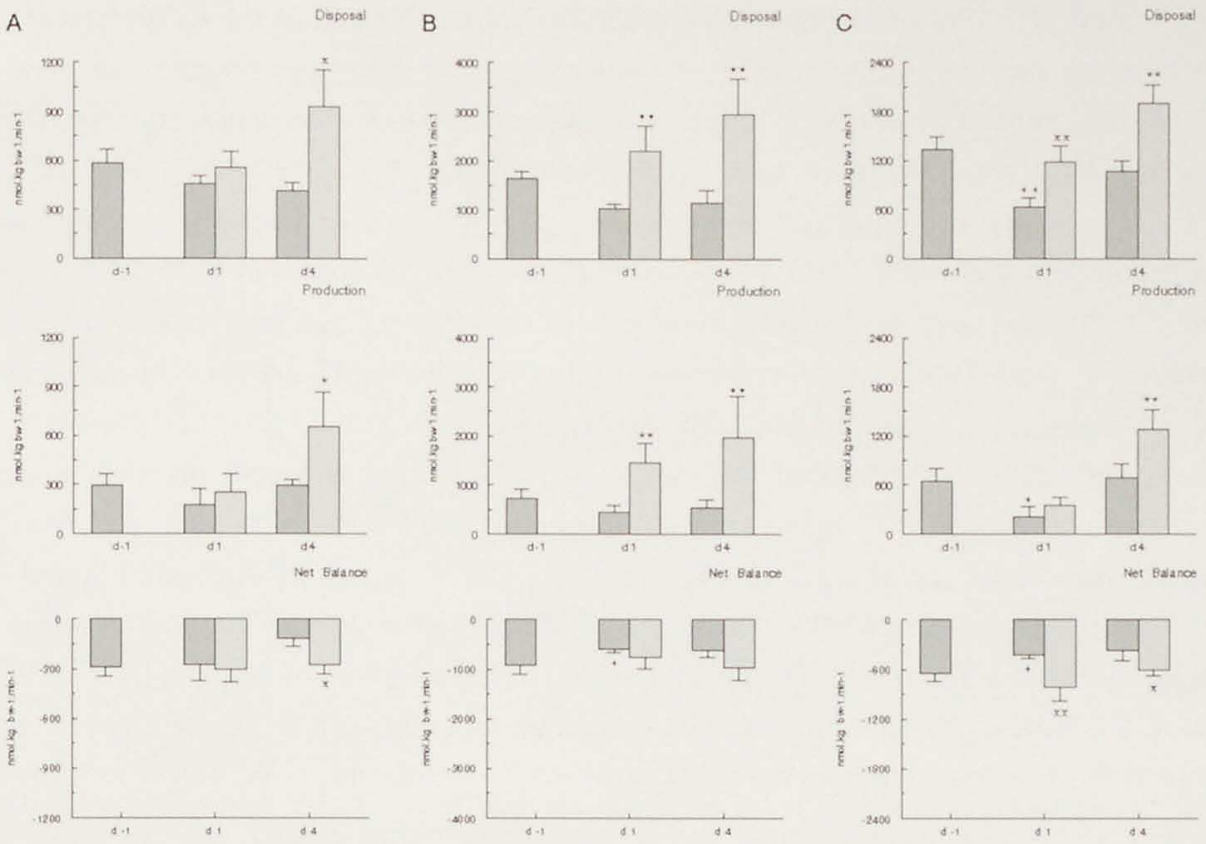

Figure 3: Disposal, production and net balance $(+=$ efflux) A: of phenylalanine (representing protein kinetics), B: of valine and $\mathrm{C}$ : of arginine across the hindquarter in fed pigs, before (d-1) and 1 and 4 after the start of saline (CON) or endotoxin (ET) infusion. Data are mean \pm SEM. Statistics by 2-way ANOVA: $P^{\star}<0.05, P^{\star \star}<0.01$, effect of ET at $d 1$ and $4 ; P^{*}<0.05, P^{+*}<0.01$, change in time; $P^{x}<0.05, P^{x x}<0.01$, interaction between ET and time.

\section{Portal-Drained Viscera (PDV)}

The ratio of the efflux of total amino acids by the PDV (Table 5) relative to the total amino acids infused by enteral infusion (Table 6 ) was higher in the ET 1 than in the CON 1 group ( 0.6 vs. 0.4 , respectively) and was also higher in the ET4 than in the CON4 group ( 0.8 vs. 0.5 , respectively). At $d 1$ after ET-treatment, the glutamate efflux by the PDV was lower, the uptake of glutamine by the PDV converted into release and efflux of most other amino acids was higher compared to control pigs (Table 5). In the ET 1 group, the total $\alpha$-AN output by the PDV was $73 \%$ higher than in the CON 1 group. The amino acid output by the PDV was still $64 \%$ higher at $\mathrm{d} 4$ after ET-treatment.

\section{Phenylalanine, valine and arginine kinetics}

Neither $1 \mathrm{~d}$, nor 4 days after ET challenge, significant changes in phenylalanine disposal (representing protein synthesis) or production of phenylalanine were observed across the PDV. The net release of phenylalanine was higher 1 day after ET than in controls. To calculate endogenous production, we corrected the measured production rate of phenylalanine for contribution of phenylalanine from the enterally infused meal (Table 6) that is escapes metabolism as illustrated in Figure 2. 
For example, the rate of disposed or metabolized phenylalanine in the CON 1 group is $791 \mathrm{nmol} \cdot \mathrm{kg} \mathrm{bw}^{-1} \cdot \mathrm{min}^{-1}$. The rate of phenylalanine from enterally absorpted is: $90 \% \times$ $1730=1557 \mathrm{nmol} \cdot \mathrm{kg} \mathrm{bw}^{-1} \cdot \mathrm{min}^{-1}$. The rate of phenylalanine of enteral nutrition that is not disposed $\left(\mathrm{P}_{N D}\right)$ and contributes to the production rate is: $1557-791=766 \mathrm{nmol} \cdot \mathrm{kg} \mathrm{bw}^{-}$ ${ }^{1} \cdot \mathrm{min}^{-1}$. Corrected for non-disposed supply, the phenylalanine derived from endogenous production $\left(\mathrm{P}_{E N D}\right)$ is: $1563-766=797 \mathrm{nmol} \cdot \mathrm{kg} \mathrm{bw}^{-1} \cdot \mathrm{min}^{-1}$ as shown in Table 7 . One day after ET infusion, higher disposal, production and efflux of valine by the PDV were observed in the ET-challenged compared to the control pigs (Table 7). The estimated endogenous valine production was also higher. Four days after ET infusion, the efflux of valine was higher compared to the control pigs. The rate of valine disposal that is transaminated in the PDV, estimated from the phenylalanine to valine molecular ratio in protein, was higher in the ET 1 than in the CON 1 group ( 1000 vs. $0 \mathrm{nmol} \cdot \mathrm{kg}$ $\left.\mathrm{bw}^{-1} \cdot \mathrm{min}^{-1}\right)$. The arginine production by the PDV was higher than in the control group 1 and 4 days post-endotoxemia (Table 7 ) resulting in higher efflux of arginine by the PDV. Also at these days, the production of estimated endogenous production of arginine was higher in the ET-treated than in the CON pigs.
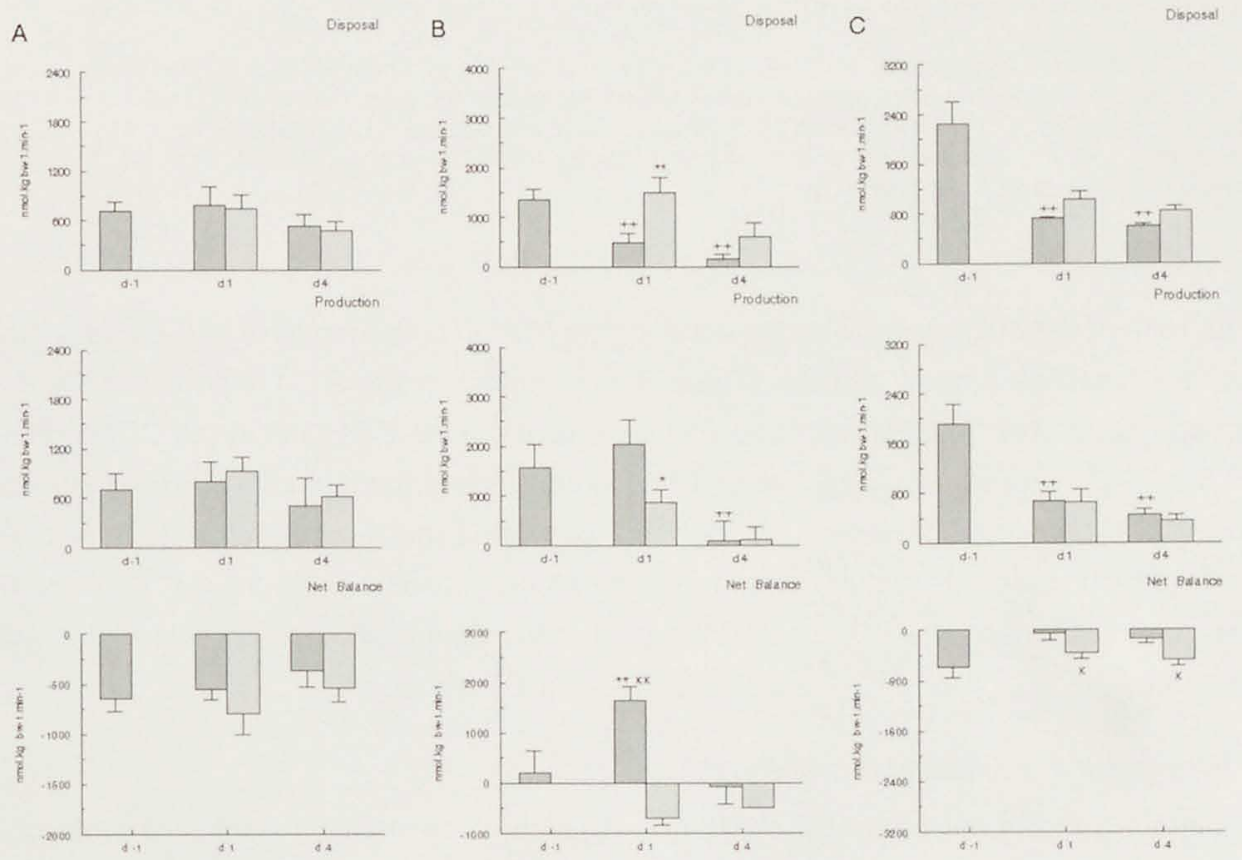

Figure $4 \mathrm{~A}$ : Disposal, production (derived from metabolism and enteral infusion) and net balance (+ = efflux) A: of phenylalanine, B: of valine (representing protein kinetics) and $C$ : of arginine across the liver in fed pigs, before $(\mathrm{d}-1)$ and 1 and 4 days after the start of saline (CON) or endotoxin (ET) infusion. Data are mean \pm SEM. Statistics by 2 -way ANOVA: $P^{*}<0.05, P^{* *}<0.01$, effect of $E T$ at $d 1$ and $4 ; P^{+}<0.05, P^{++}<0.01$, change in time; $P^{x}<0.05, P^{x \times}<0.01$, interaction between $E T$ and time. 
Table 7. Disposal, production and for enteral infusion corrected production and net balance of phenylalanine (representing protein kinetics), valine and arginine across the portal-drained viscera in fed pigs, before (d -1$)$ and 1 and 4 days after the start of saline (CON) or endotoxin (ET) infusion.

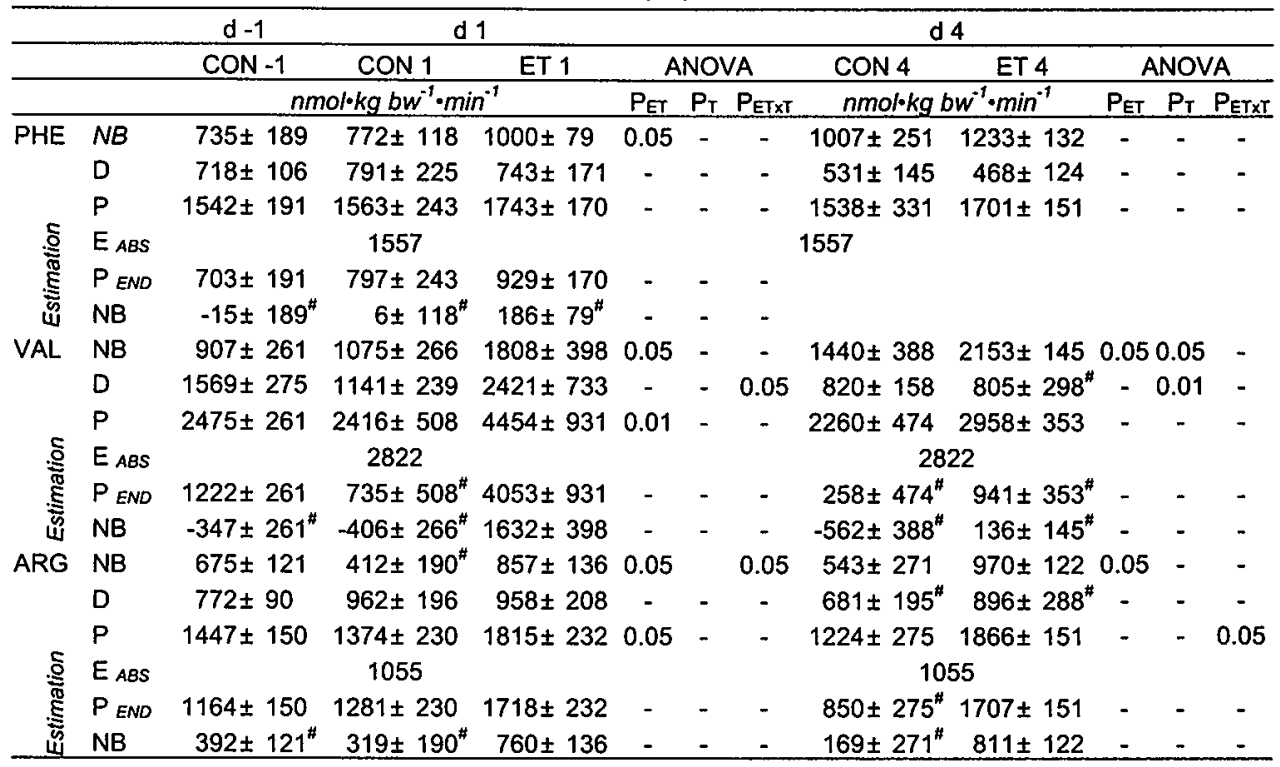

Abbreviations used: bw, body weight; NB, net balance; $D$, disposal; $P$, measured production derived from endogenous production plus enteral infusion; $E_{A B S}$, enterally absorption estimated from $90 \%$ the enteral infusion; $P_{E N D}$, calculated endogenous production; $N_{E N D}$, calculated endogenous net balance. Calculations are made accordingly to Figure 2. Data are means \pm SEM. Statistics by 2-way ANOVA: $p$ Values of significance indicate probability of differences: $P_{E T}$, between CON and ET at $d 1$ and $4 ; P_{T}$, within CON between d 1 or 4 and -1 ; $P_{E T}$, interaction between ET and time. Wilcoxon test: "not different from zero.

Table 8. Net balance (NB= flux) across the liver in fed pigs, before $(d-1)$ and 1 and 4 days after the start of saline (CON) or endotoxin (ET) infusion.

\begin{tabular}{|c|c|c|c|c|c|c|c|c|c|c|c|}
\hline & \multirow{2}{*}{$\frac{d-1}{\operatorname{CON}-1}$} & \multicolumn{2}{|c|}{ d 1} & \multicolumn{8}{|c|}{$d 4$} \\
\hline & & CON 1 & ET 1 & \multicolumn{3}{|c|}{ ANOVA } & $\mathrm{CON} 4$ & ET 4 & \multicolumn{3}{|c|}{ ANOVA } \\
\hline & \multicolumn{3}{|c|}{$n m o l \cdot k a b w^{-1} \cdot m^{-1}$} & $\mathrm{PEr}$ & Pr & $P_{E Y X Y}$ & \multicolumn{2}{|c|}{$n m o l \cdot k g b w^{-1} \cdot m^{-1}$} & $P_{E T}$ & $P_{T}$ & $P_{E I X I}$ \\
\hline Glutamate & $7840 \pm 785$ & $4052 \pm 564$ & $8282 \pm 123$ & - & - & - & $4141 \pm 553$ & $4987 \pm 675$ & - & - & - \\
\hline Glutamine & $-3041 \pm 583$ & $155 \pm 1 Q 5$ & $-2758 \pm 582$ & 0.050 & 0.05 & - & $342 \pm 668^{\#}$ & $-1657 \pm 653$ & 0.05 & - & - \\
\hline Glycine & $-3103 \pm 223$ & $-848 \pm 158^{\#}$ & $-1486 \pm 695^{\#}$ & $7-$ & - & - & $-120 \pm 504^{\#}$ & $-40 \pm 317^{\#}$ & - & - & - \\
\hline Arginine & $153 \pm 105^{\#}$ & $85 \pm 50^{\#}$ & $120 \pm 76^{*}$ & - & - & - & $-2 \pm 70^{*}$ & $-98 \pm 107^{*}$ & - & - & - \\
\hline Alanine & $-580 \pm 165$ & $-246 \pm 117^{\#}$ & $-372 \pm 84$ & - & - & - & $-147 \pm 73^{\#}$ & $-481 \pm 92$ & 0.01 & - & - \\
\hline Tyrosine & $-4483 \pm 769$ & $-1215 \pm 662^{\#}$ & $-1606 \pm 258$ & - & - & - & $-1189 \pm 615^{\#}$ & $-2393 \pm 110$ & - & - & - \\
\hline Valine & $-916 \pm 251$ & $-375 \pm 385^{\#}$ & $-1317 \pm 241$ & 0.05 & - & - & $-149 \pm 231^{\#}$ & $-759 \pm 251$ & 0.05 & - & - \\
\hline Isoleucine & $200 \pm 438^{\#}$ & $1638 \pm 276$ & $-709 \pm 128$ & 0.01 & - & 0.01 & $-73 \pm 348^{\#}$ & $-484 \pm 12$ & 0.01 & - & 0.01 \\
\hline Phenylalanin & $-259 \pm 300^{\#}$ & $1111 \pm 359^{\#}$ & $-594 \pm 181$ & 0.01 & - & - & $82 \pm 89^{*}$ & $-602 \pm 187$ & 0.05 & - & - \\
\hline Leucine & $-641 \pm 134$ & $-553 \pm 100$ & $-800 \pm 203$ & - & - & - & $-365 \pm 165^{\#}$ & $-540 \pm 136$ & - & - & - \\
\hline Lysine & $-155 \pm 166^{\#}$ & $-325 \pm 227^{\#}$ & $-178 \pm 158$ & - & - & - & $82 \pm 131^{\prime \prime}$ & $-249 \pm 111^{\#}$ & - & - & - \\
\hline & & $n o l \cdot k g b w^{-1} \cdot m i$ & & & & & $\mu \mathrm{mol} / \mathrm{kg} \mathrm{b}$ & 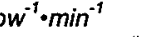 & & & \\
\hline BCAA & $-0.9 \pm 1.2^{\#}$ & $2.8 \pm 0.5$ & $-2.6 \pm 0.7$ & 0.01 & - & - & $1.0 \pm 0.4^{\#}$ & $-1.6 \pm 1.8^{\#}$ & - & - & - \\
\hline$\alpha-A N$ & $-10.4 \pm 4.8^{\#}$ & $1.9 \pm 3.7^{\#}$ & $-6.4 \pm 1.8$ & 0.05 & - & - & $3.5 \pm 2.6^{\#}$ & $-2.6 \pm 3.4^{\#}$ & 0.05 & 0.05 & - \\
\hline Glucose & $20.9 \pm 3.2$ & $21.2 \pm 2.2$ & $19.2 \pm 3.9$ & 0.01 & - & - & $21.5 \pm 2.9$ & $21.6 \pm 2.0$ & - & - & - \\
\hline Lactate & $-6.2 \pm 1.9$ & $-8.0 \pm 2.5$ & $-17.7 \pm 3.7^{\#}$ & 0.01 & - & - & $-7.2 \pm 1.0$ & $-5.2 \pm 1.9$ & - & - & - \\
\hline Urea & $8.3 \pm 1.3$ & $6.6 \pm 0.6$ & $1.5 \pm 1.9^{*}$ & - & - & - & $6.4 \pm 2.3$ & $5.1 \pm 2.9$ & - & - & - \\
\hline
\end{tabular}

Abbreviations used: see Table 4. Data are means \pm SEM. $+=$ Net efflux or release. Statistics by 2-way ANOVA: $P$ values of significance indicate probability of differences: $P_{E T}$, between CON and ET at $d 1$ and 4; PT, within CON between $d$ or 4 and $d-1$; PETxT, interaction between ET and time. Wilcoxon test: "not different from zero. 


\section{Liver}

A shift of efflux into influx of glutamine, valine and isoleucine in the ET 1 and 4 group was observed (Table 8). One days after ET-treatment the uptake of lactate by the liver was significantly higher compared to CON pigs.

\section{Phenylalanine, valine and arginine kinetics}

One and 4 days after ET challenge, the phenylalanine disposal (protein synthesis plus hydroxylation) and production (protein degradation) by the liver did not significantly differ between the ET and the CON groups (not shown). No differences in the estimated rate of phenylalanine hydroxylation between the two groups were observed at $d 1$ and 4 (not shown). Within the CON group, the disposal of valine decreased at $d 1$ and 4 compared to control $d-1$ (Figure 4A). At $d 1$ after the ET infusion, the disposal of valine (protein synthesis) was higher and the production of valine (protein degradation) was lower compared to the CON 1 group net resulting in uptake of valine across the liver. Both the disposal and production of arginine by the liver decreased at $d 1$ and 4 compared to $d-1$ (Figure 4B). In comparison to CON pigs, ET had a time-related effect by increasing the hepatic disposal of arginine 1 and 4 days post-endotoxemia resulting in higher net uptake of arginine.

\section{Discussion}

Muscle wasting, intestinal permeability and enhanced liver protein synthesis are wellknown characteristics of the septic state that are of prolonged duration in fasting conditions. Preventing protein loss is one of the many reasons to favor a policy of early enteral feeding to critically ill patients $(23,24)$. Under starved circumstances, nutritional supply proved beneficial by promoting protein synthesis rates (28). The exact manner in which organ protein metabolism is altered after a period of endotoxemia under conditions of adequate nutritional substrates, however, remains unclear. In this study, the changes in protein synthesis and breakdown and the amino acid degradation across the main organs was explored to better understand adaptations of protein and amino acid metabolism in a post-endotoxemic period during nutritional intervention. It has to be taken into account that protein turnover in pig is higher compared to man $(18,19)$ what, together with the use of a moderate sub-acute model of sepsis, may lead to relative rapid manifestation of the endotoxemia-induced modifications in protein metabolism under feeding conditions.

In this post-sepsis model, many of the observed changes we observed were attributable to both endotoxin intervention and changes over time. Differences that exist between $d$ 1 and $d 1$ or 4 within the control group may result from the extensive experimental 
procedure during which all the treatments of the endotoxin-treated pigs were given to control pigs, except for endotoxin. We suppose that the flow and flow-related rates of disposal, production and flux dropped because of the procedure the control pigs were subjected to.

Across the hindquarter muscle, 1 day after endotoxemia during feeding, no significant changes in phenylalanine disposal or production occurred, indicating unchanged muscle protein metabolism. In a more prolonged phase of endotoxemia at $d 4$, protein turnover appeared to increase in such a way that the increase in protein synthesis exceeded the increase in protein degradation resulting in net protein anabolism. The increased protein synthesis possibly represents replenishment and renovation of wasted proteins. In the muscle under anabolic circumstances, amino acids (except for glutamine) are not only used for protein synthesis but also partly transaminated. In muscle, amino acids, especially the BCAA, can either be transaminated producing glutamine and alanine, involving glutamate as an intermediate or can be completely oxidized for energy supply after transamination (31). One day after endotoxemia, the amount of valine disposal used in transamination in muscle estimated from phenylalanine to valine ratio in protein was elevated. An increased glutamine and alanine efflux in parallel with an increased disposal of valine and net consumption of glutamate, suggest increased transamination activity in muscle during the acute and the prolonged phase after endotoxemia. Glucose provides most of the pyruvate used for alanine synthesis (27). However, the endotoxin infusion period had no effect on the giucose consumption or lactate production by the hindquarter. In addition, the net uptake of alanine decreased to a much lesser extent than the net release of glutamine in the post-endotoxemia period, suggesting a minor role for the glycolytic pathway during feeding. After endotoxemia, muscle may both use glutamate and BCAA from increased inward transport and from increased protein breakdown preferentially for glutamine than for alanine production.

Arginine is considered an indispensable amino acid under disease conditions like sepsis and its availability to support immune function (39) and protein homeostasis (41) may become limiting under such circumstances. Considering the major route of entry of arginine into the plasma is via its release from proteins (2), the increased release of arginine from muscle catabolism during endotoxemia has led to depletion of intramuscular arginine. Therefore, the observed enhanced net uptake of arginine by the hindquarter may serve protein synthesis and reflect replenishment of the muscle arginine pool.

Studies dealing with intestinal protein metabolism during sepsis are relatively scarce and few data of the long-term effects of sepsis on intestinal protein metabolism are available. Studies using long-term models of sepsis in rats yield contradictory results since both enhanced (9) and decreased intestinal protein synthesis (11) were reported. In this post- 
sepsis model, protein synthesis and degradation in the PDV were not significantly affected during feeding at 1 and 4 days after endotoxemia. Compromised integrity of the gut wall and decreased intestinal transit, which are known to be affected by endotoxin for several days (36), could have resulted in decreased amino acid retention and subsequent increased amino acid release by the PDV. The assumption that intestinal absorption of amino acids from enteral supply is $90 \%$ may not apply for the endotoxemia group. Nevertheless, a higher efficiency of absorption can not explain the $60-70 \%$ higher output of amino acids occurring across the PDV of the ET-treated pigs. Moreover, since many studies showed substrate absorption rather to impair than to enhance under septic conditions (17), this concept seems doubtful.

In the small intestine, BCAA are catabolized by transamination involving alanine production and the utilization of glutamine or glutamate or by further oxidation to $\mathrm{CO}_{2}$ (38). We observed that, 1 day after endotoxin-treatment in pigs, alanine was released to a larger extent accompanying decreased release of glutamate. Besides decreased glutamate efflux, the PDV appear to acquire substrate for alanine production by increasing valine disposal. Possibly, BCAA together with glutamate are increasingly metabolized in the gut 1 day after endotoxemia resulting in alanine production. If glutamine synthesis acts as a substrate involved in the increased transamination activity, this explains the observed reduced influx of glutamine across the PDV in our postendotoxemia model. The estimated rate of valine used for transamination relative to protein synthesis in the PDV increased 1 day post-endotoxemia. This observation suggests that BCAA that are made available by disposal are increasingly used for the transamination process. Glucose derived pyruvate can be used for alanine synthesis or energy requirements, however increased uptake of glucose was not observed. Whereas under conditions of fasted endotoxemia the gut glucose disposal is increased (25), during enteral nutrition the glucose supply will be sufficient to foresee in increased demands by the PDV.

In the liver, the net protein anabolism that occurred during the acute and prolonged phase after endotoxemia resulted from both increased protein synthesis and decreased protein degradation. Although the hepatic uptake of lactate was increased 1 day after endotoxin-treatment, the hepatic output of glucose was not altered. In addition, the glucose production of the whole body was not changed after endotoxemia. This implied that no significant elevated hepatic gluconeogenesis rates were present likely due to high rates of glucose provided by enteral feeding. The augmented amino acid consumption by liver occurring 1 and 4 days after endotoxemia therefore appears to serve increased protein synthesis rather than gluconeogenesis. The increase in hepatic protein synthesis possibly represents the synthesis of acute-phase proteins in the acute-phase response that can last for several days (20). Under fed circumstances, the major site of amino acid 
degradation to urea is the liver, glutamate in this process acting as intermediate. Opposite to the control pigs, we observed a quantitative release of hepatic glutamate into the circulation 1 and 4 days post-endotoxemia. This glutamate possibly derives from conversion of amino acids that are increasingly consumed by the liver. A shift of nitrogen released as urea towards glutamate seems apparent in liver after endotoxemia. Glutamate can serve an important role as carbon and nitrogen carrier between organs.

Whereas the sudden effect of endotoxin on skeletal muscle normally constitutes accelerated protein degradation $(4,10)$, no muscle catabolism was manifest during nutritional intervention as soon as 1 day after endotoxemia. The long-term effect of sepsis included increased protein turnover in muscle resulting in increased net protein synthesis, possibly reflecting a recovery mechanism of the muscle to regain protein mass. The effect of endotoxemia also comprises the enhanced transamination of BCAA with glutamate into alanine but predominantly, glutamine. The need for glutamine for different processes may be sustained after endotoxemia. The need for alanine in the liver for gluconeogenesis is relatively low since glucose is no longer a limiting fuel in the anabolic state. The increased rates of amino acid retained by the liver at both the acutephase and the prolonged-phase after endotoxemia seem for the purpose of hepatic protein synthesis. In the acute and more prolonged phase after endotoxemia, the increased amino acid release by the PDV area that possibly results from impaired amino acid retention by the gut caused an increased portal output of amino acids. In combination with the process of increased transamination, the PDV makes more amino acids, specifically glutamine, glycine, alanine and arginine available for protein synthesis in liver and other consuming organs, e.g., immune-cell rich tissues and kidneys during the post-endotoxemia recovery period. The arginine production by PDV and hindquarter predominantly account for the increase in whole body production of arginine. Nevertheless, lower arginine plasma levels were present indicating elevated need for arginine by organs like the liver and hindquarter.

The carbon cycle (Felig cycle) of glucose and glutamine/alanine between periphery and PDV on the one site and liver on the other site, seems quantitatively more important in the fed condition than the carbon cycle (Cori cycle) of lactate and glucose. Organ protein metabolism after endotoxemia in the fed state is modified in such a way that net amino acid release by the PDV is enhanced whereas in muscle and liver amino acid uptake for net protein synthesis predominates. Elevated rates of amino acid release, from the transamination process in both PDV and muscle, may serve in providing substrate for the synthesis of various plasma proteins in liver and immune cells that are of importance for sustained tissue repair and host defense after endotoxemia. Therefore, it seems that in acute and prolonged post-endotoxemic conditions, the PDV and hindquarter are entirely in service of the liver and, possibly, the immune system by releasing amino acid 
precursors used in recovery mechanisms. In such situations, substrate supply by nutritional intervention can assist the altered organ protein metabolism in a rapid recovery.

\section{Acknowledgements}

The authors thank Mr. H.M.H. van Eijk and Mr. J.L.J.M. Scheijen for analytical amino acid measurements and Mrs. G.A.M. ten Have for assistance during operational and experimental procedures.

\section{References}

1. Arnold, J., I. T. Campbell, T. A. Samuels, J. C. Devlin, C. J. Green, L. J. Hipkin, I. A. MacDonald, C. M. Scrimgeour, K. Smith, and M. J. Rennie. Increased whole body protein breakdown predominates over increased whole body protein synthesis in multiple organ failure. Clin Sci (Colch) 84: 655-61, 1993.

2. Beaumier, L., L. Castillo, Y. M. Yu, A. M. Ajami, and $V$. R. Young. Arginine: new and exciting developments for an "old" amino acid. Biomed Environmental Sci 9: 296-315, 1996.

3. Biolo, G., R. Y. D. Fleming, S. Maggi, and R. R. Wolfe. Transmembrane transport and intracellular kinetics of amino acids in human skeletal muscle. Am J Physiol 268: E75-E84, 1995.

4. Biolo, G., G. Toigo, B. Ciocchi, R. Situlin, F. Iscra, A. Gullo, and G. Guarnieri. Metabolic response to injury and sepsis: changes in protein metabolism. Nutrition 13: 52S-57S, 1997.

5. Bower, R. H. Nutrition during critical illness and sepsis. New Horiz 1: 348-52., 1993.

6. Braga, M., L. Gianotti, G. Radaelli, A. Vignali, G. Mari, O. Gentilini, and V. Di Carlo. Perioperative immunonutrition in patients undergoing cancer surgery: results of a randomized double-blind phase 3 trial. Arch Surg 134: 428-33, 1999.

7. Braga, M., L. Gianotti, A. Vignali, A. Cestari, P. Bisagni, and V. Di Carlo. Artificial nutrition after major abdominal surgery: impact of route of administration and composition of the diet [see comments]. Crit Care Med 26: 24-30, 1998.

8. Breuille, D., M. Arnal, F. Rambourdin, G. Bayle, D. Levieux, and C. Obled. Sustained modifications of protein metabolism in various tissues in a rat model of long-lasting sepsis. Clin Sci (Colch) 94: 413-23, 1998.

9. Breuillé, D., M. Arnal, F. Rambourdin, G. Bayle, D. Levieux, and C. Obled. Sustained modifications of protein metabolism in various tissues in a rat model of long-lasting sepsis. Clin Sci 94: 413-423, 1998.
10. Bruins, M. J., P. B. Soeters, and N. E. P. Deutz. Inter-organ protein metabolism in a hyperdynamic model of sepsis and starvation in the pig. submitted.

11. Cooney, R. N., E. Owens, D. Slaymaker, and T. C. Vary. Prevention of skeletal muscle catabolism in sepsis does not impair visceral protein metabolism. Am J Physiol 270: E621E626, 1996.

12. Deutz, N. E., C. H. Dejong, G. Athanasas, and P. B. Soeters. Partial enterectomy in the rat does not diminish muscle glutamine production. Metabolism 41: 1343-50, 1992.

13. Deutz, N. E. P., M. J. Bruins, and P. B. Soeters. Infusion of soy and casein protein meals affect interorgan amino acid metabolism and urea kinetics differently in pigs. $J$ Nutr 128: 2435-2445, 1998.

14. Deutz, N. E. P., P. L. M. Reijven, G. Athanasas, and P. B. Soeters. Post-operative changes in hepatic, intestinal, splenic and muscle fluxes of amino acids and ammonia in pigs. Clin Sci 83: 607-614, 1992.

15. Dodds, W. J. The pig model for biomedical research. Federation Proc 41: 247-256, 1982.

16. Fink, M. P., and S. O. Heard. Laboratory models of sepsis and septic shock. J Surg Res 49: 186-196, 1990.

17. Gardiner, K. R., R. E. Gardiner, and A. Barbul. Reduced intestinal absorption of arginine during sepsis. Crit Care Med 23: 1227-1232, 1995.

18. Garlick, P. J., T. L. Burk, and R. W. Swick. Protein synthesis and RNA in tissues of the pig. Am J Physiol 230: 1108-12, 1976.

19. Garlick, P. J., and M. I. A technique for measuring brain protein synthesis. $J$ Neurochem 19: 577-583, 1972. 
20. Heegaard, P. M., J. Klausen, J. P. Nielsen, N. Gonzalez-Ramon, M. Pineiro, F. Lampreave, and $M$. A. Alava. The porcine acute phase response to infection with Actinobacillus pleuropneumoniae. Haptoglobin, C-reactive protein, major acute phase protein and serum amyloid $A$ protein are sensitive indicators of infection. Comp Biochem Physiol B Biochem Mol Biol 119: 365-73, 1998.

21. Jackson, N. C., P. V. Carroll, D. L. RussellJones, P. H. Sonksen, D. F. Treacher, and A. $M$. Umpleby. The metabolic consequences of critical illness: acute effects on glutamine and protein metabolism. Am J Physiol 276: E16370, 1999.

22. Jeevanandam, M., R. F. Shamos, and S. R. Petersen. Substrate efficacy in early nutrition support of critically ill multiple trauma victims. $J$ Parenter Enteral Nutr 16: 511-20, 1992.

23. Kudsk, K. A. Gut mucosal nutritional support-enteral nutrition as primary therapy after multiple system trauma. Gut 35: S52-4, 1994.

24. Mainous, M. R., E. F. Block, and E. A. Deitch. Nutritional support of the gut: how and why. New Horiz 2: 193-201, 1994.

25. Meszaros, K., C. H. Lang, G. J. Bagby, and J. J. Spitzer. In vivo glucose utilization by individual tissues during nonlethal hypermetabolic sepsis. Faseb $J$ 2: 3083-6, 1988.

26. Miller, E. R., and D. E. Ullrey. The pig as a model for human nutrition. Annu Rev Nutr 7: 361-82, 1987.

27. Newsholme, E. A., and A. R. Leech. Biochemistry for the Medical Sciences. New York: John Wiley \& Sons, 1983. p. 1-952.

28. Ogata, E. S., S. K. Foung, and M. A. Holliday. The effects of starvation and refeeding on muscle protein synthesis and catabolism in the young rat. J Nutr 108: 759-65, 1978.

29. Ooiwa, T., H. Goto, Y. Tsukamoto, T. Hayakawa, S. Sugiyama, N. Fujitsuka, and $Y$ Shimomura. Regulation of valine catabolism in canine tissues: tissue distributions of branchedchain aminotransferase and 2-oxo acid dehydrogenase complex, methacrylyl-CoA hydratase and 3-hydroxyisobutyryl-CoA hydrolase. Biochim Biophys Acta 1243: 216220, 1995.
30. Pond, W. G. Life cycle feeding: maternal nutrition and progency development. In: Swine in biomedical research. New York: Plenum press, 1986, p. 915-930.

31. Skeie, B., V. Kvetan, K. M. Gil, M. M. Rothkopf, E. A. Newsholme, and J. Askanazi. Branchchain amino acids: their metabolism and clinical utility. Crit Care Med 18: 549-571, 1990.

32. Ten Have, G. A. M., M. C. F. Bost, J. C. A. W. Suyk-Wierts, A. E. J. M. van den Bogaart, and N. E. P. Deutz. Simultaneous measurement of metabolic flux in portally-drained viscera, liver, spleen, kidney and hindquarter in the conscious pig. Lab Anim 30: 347-358, 1996.

33. Tourian, A., J. Goddard, and T. T. Puck. Phenylalanine hydroxylase activity in mammalian cells. J Cell Physiol 73: 159-170, 1969.

34. Van Eijk, H. M. H., D. R. Rooyakkers, and N. E. $P$. Deutz. Determination of amino acid isotope enrichment using liquid chromatography-mass spectrometry. Anal Biochem 271: 8-17, 1999.

35. Van Eijk, H. M. H., D. R. Rooyakkers, and N. E. P. Deutz. Rapid routine determination of amino acids in plasma by high-performance liquid chromatography with a 2-3 $\mu \mathrm{M}$ Spherisorb ODS II column. J Chromatogr 620: 143-148, 1993.

36. Wang, Q., N. Pantzar, B. Jeppsson, B. R. Westrom, and B. W. Karisson. Increased intestinal marker absorption due to regional permeability changes and decreased intestinal transit during sepsis in the rat. Scand $J$ Gastroenterol 29: 1001-1008, 1994.

37. Wolfe, R. R. Radioactive and stable isotope tracers in biomedicine. Principles and practice of kinetic analysis. New York: Wiley-Liss, 1992.

38. Wu, G. Intestinal mucosal amino acid catabolism. J Nutr 128: 1249-1252, 1998.

39. $\mathrm{Wu}, \mathrm{G}$, and S. M. Morris, Jr. Arginine metabolism: nitric oxide and beyond. Biochem J 336: 1-17, 1998.

40. Wu, G., T. L. Ott, D. A. Knabe, and F. W Bazer. Amino acid composition of the fetal pig. J Nutr 129: 1031-8, 1999.

41. Yu, Y.-M., R. L. Sheridan, J. F. Burke, T. E. Chapman, R. G. Tompkins, and V. R. Young. Kinetics of plasma arginine and leucine in pediatric burn patients. Am J Clin Nutr 64: 6066, 1996. 

L-ARGININE SUPPLEMENTATION IN THE PIG DECREASES LIVER PROTEIN TURNOVER AND INCREASES HINDQUARTER PROTEIN TURNOVER BOTH DURING AND AFTER ENDOTOXEMIA

\author{
Maaike J Bruins \\ Peter $B$ Soeters \\ Wouter H Lamers * \\ Nicolaas EP Deutz
}

Department of Surgery, Maastricht University, The Netherlands

- Department of Anatomy \& Embryology, University of Amsterdam, AMC, The Netherlands 


\section{Abstract}

Background: Accumulating evidence suggests that L-arginine under conditions of septicemia not only enhances immune function but also improves protein metabolism. Objective: Because the effect of L-arginine administration on the protein metabolism of the different organs is unknown, the aim of the study was to elucidate the effects of exogenous supplementation of L-arginine during endotoxemia on the in vivo protein metabolism of individual organs and at whole body level. Design: Female pigs were cannulated with catheters in the aorta, the splenic-, caval-, portal-, hepatic- and renal vein, enabling measurements across the hindquarter, portal-drained viscera, liver and kidneys. Endotoxemia was induced by a 24-h continuous intravenous infusion of endotoxin $\left(3 \mu \mathrm{g} \cdot \mathrm{kg}^{-1} \cdot \mathrm{h}^{-1}\right)$. At $8 \mathrm{~h}$, intravenous infusion of L-arginine was started $(n=8)$. Control pigs $(n=6)$ received L-alanine. At $24-h$, blood was sampled. After cessation of endotoxin, L-arginine and L-alanine infusions were continued as supplement in the enterally infused diet. At 48-h, samples of the postendotoxemic and nutritionally supported condition were obtained. Stable isotopes were used to assess protein metabolism and phenylalanine hydroxylation. Results: Both during and after endotoxin challenge, L-arginine administration enhanced protein synthesis and degradation across the hindquarter and simultaneously reduced the liver protein synthesis and degradation at equal rates. Protein turnover across the kidneys and portal-drained viscera remained unaffected. After endotoxemia, L-arginine infusion decreased the whole body protein turnover without affecting the net protein balance. Conclusions: The results of this (post)endotoxemia study demonstrate that L-arginine administration affects protein turnover of the muscle-area and the liver oppositely.

\section{Introduction}

L-Arginine plays a pivotal role in many functions including its role in protein synthesis and breakdown (reviewed in: (1)). In stress situations such as injury, burn and sepsis, Larginine may become an indispensable amino acid (35). In animals and humans with sepsis, plasma L-arginine concentrations are markedly decreased and low L-arginine concentrations are correlated with a worse prognosis in septic patients (18). In times of stress or severe injury, the endogenous supply of L-arginine may become critical for sustenance of homeostasis. Dietary administrated L-arginine has been shown to enhance several functions including immune defense (11) and wound healing (7). Under catabolic conditions, L-arginine was ascribed anti-catabolic properties by improving impaired nitrogen balance $(7,8,28,30)$. Moreover, L-arginine can be involved in protein metabolism through its metabolites. L-Ornithine serves as precursor in the synthesis of 
polyamines that are required for DNA synthesis and cell growth (19), and of proline, which is used in collagen synthesis (7). Dietary supplementation with L-ornithine or Lornithine analogues was demonstrated to exert anti-catabolic or anabolic effects (for overview see: (12)). L-Arginine-derived nitric oxide (NO), on the other hand, seems involved in decreased protein synthesis in vitro $(9,15,22)$.

L-Arginine supplemented dietary formulas used in clinical studies suggest a significant benefit for injured patients not only by improved immune function, but also by improved nitrogen balance $(3,4,36)$. These formulas, however, consist of multiple supplements, this composition not allowing discrimination of the effect of L-arginine as an individual component.

To our opinion, it remains to be elucidated whether L-arginine supplementation in critical illness is capable of modifying protein metabolism. Therefore, we assessed the effect of L-arginine supplementation on protein metabolism of the portal-drained viscera, liver, hindquarter and kidney in this model of endotoxemia.

Because generally in human sepsis endotoxin is slowly released from the gut (21) and only low doses of endotoxin are detected in the circulation (6), we conducted a study in pigs infusing a low dose of endotoxin for several hours. We hypothesized that exogenous L-arginine administration during the initial phase of sepsis could possibly induce excessive production of NO. Therefore, L-arginine was started a few hours after initiation of endotoxin infusion to prevent NO-mediated hypotension and shock.

The aim of this research was to investigate the effects of intravenously (I.V.) administered L-arginine intervention during endotoxemia and intragastrically supplied Larginine after endotoxemia on protein metabolism. The use of tracer methodology in a multi-catheterized animal model enabled us to not only investigate the changes in whole body protein synthesis and breakdown at a whole body level but also across the individual organs. To our knowledge, the influence of L-arginine treatment during or after endotoxemia on organ protein metabolism has not previously been performed.

\section{Materials and methods}

\section{Animals}

Pigs were offsprings of Yorkshire $x$ Dutch Landrace. Three-month old pigs, weighting 20$22 \mathrm{~kg}$ body weight (bw) were individually housed. Pigs were given $1 \mathrm{~kg}$ of regular pig feed ( $149 \mathrm{~g}$ crude protein $/ \mathrm{kg}$; Landbouwbelang, Roermond, the Netherlands; $16 \%$ crude protein) daily, which supported a growth rate of approximately 300 gram per day. Before 
surgery, pigs were randomly assigned to one of the two treatment groups. The Animal Ethics Committee of the Maastricht University approved the study.

\section{Surgical procedure}

The night before surgery, the pigs were withheld from food. One hour after premedication with $10 \mathrm{mg} / \mathrm{kg}$ bw intramuscular azoperone (Stresnil, Janssen pharmaceutica, Beersse, Belgium), anesthesia was induced by a mixture of $\mathrm{N}_{2} \mathrm{O} / \mathrm{O}_{2}(1: 2)$ and halothane $(0.8 \%)$. After intubation, the pigs were intravenously administrated $6.25 \mathrm{mg} / \mathrm{kg} \mathrm{bw}$ lincomycin. $2 \mathrm{HCl}$ (Lincomycin, A.U.V., Cuyk, the Netherlands) as bactericidal prophylaxis and $12.5 \mathrm{mg} / \mathrm{kg}$ bw spectinomycin. $\mathrm{HCl}$ lyophil (Spectinomycin, A.U.V.) as bacteriostatic prophylaxis. To avoid coagulation and as postoperative analgesia, $50 \mathrm{mg} / \mathrm{kg}$ bw flunixine (Finadyne, Schering-Ploegh, Brussel, Belgium) was given. During surgery, the anesthesia was maintained by the $\mathrm{N}_{2} \mathrm{O} / \mathrm{O}_{2}$-halothane mixture and I.V. given Lactetrol (per ml: $5,76 \mathrm{mg} \mathrm{NaCl}, 0.37 \mathrm{mg} \mathrm{KCl}, 0.37 \mathrm{mg} \mathrm{CaCl}$, $0.2 \mathrm{mg} \mathrm{MgCl}_{2}, 5 \mathrm{mg}$ sodium lactate, methyl parahydroxy bezoicacid, Janssen pharmaceutica). The surgical procedure has been described in detail $(14,31)$. In summary, 8 vessels were cannulated after a midline incision. Two catheters were inserted in the abdominal aorta; one at a level just above the bifurcation (A1) and one at a level above the right renal vein (A2) and, similarly, two in the inferior caval vein (V1 and V2, respectively). Furthermore, catheters were inserted in the portal $(P)$, hepatic $(H)$, splenic $(S)$ and renal $(R)$ vein. The $A 1$ and the $S$ catheters were used for the infusion of para-aminohippuric acid $(\mathrm{PAH})$ for determination of plasma flow and the V2 catheter for isotope and endotoxin infusion. For portal-drained viscera, splanchnic, hindquarter and renal fiux measurements, blood sampled from the $P, H, V 1$ and $\mathrm{R}$ catheters, was used respectively. Also, a gastrostomy catheter was inserted in the stomach to enable infusion of a liquid diet. All catheters were tunneled through the abdominal wall and skin.

The pigs wore a canvas harness to protect the catheters, stoma and to allow easy handling. The first day post-operatively, the pigs were fed $100 \mathrm{~g}$, the second day $200 \mathrm{~g}$ and from the third day onwards, $1 \mathrm{~kg}$ of food daily. To avoid catheter tip infections and to maintain patency, catheters were regularly flushed with $150 \mathrm{mmol} / \mathrm{L} \mathrm{NaCl}$ (saline) and filled with a solution containing a mixture of gentamycin sulfate $(20 \mathrm{~g} / \mathrm{L}$, Gentamycin $5 \%$, A.U.V.) and chymotrypsin ( $0.225 \mathrm{U} / \mathrm{L}$, Merck, Darmstadt, Germany) in saline.

\section{Postoperative care}

Postoperative care was standardized (31). A canvas harness was fitted to each pig to protect the catheters and to allow easy handling of the animal. During the procedure of postoperative care, the pigs were placed in a movable cage to get accustomed to their experimental condition. The procedure of postoperative care of the pigs was 
standardized, as has been described previously (14). During the whole recovery (10-15 days) and experimental period ( 7 days), the animals remained healthy without signs of infection. Four weeks after the operation, the position of the catheter tips were checked under anesthesia using fluoroscopy.

\section{Experimental protocol}

From the tenth postoperative day onwards a ready-to-use liquid diet (per $1 \mathrm{~L}$ liquid food: $63 \mathrm{~g}$ casein protein, $141 \mathrm{~g}$ carbohydrates; $11 \mathrm{~g}$ sugars, $128 \mathrm{~g}$ polysaccharides, $49 \mathrm{~g}$ fat, minerals, trace elements and vitamins, osmolarity $255 \mathrm{mOsmol} / \mathrm{L}$; Nutrison Steriflo Protein-Plus, Nutricia, Zoetermeer, the Netherlands) was intragastrically infused into the gastrostomy catheter via a swivel system. This meal is a high-protein liquid meal with an optimal protein/energy ratio, containing all vitamins, minerals and other nutritional elements that meet the requirements for clinical patients but also for growing pigs (27). To get the pigs accustomed to the diet, the first day enteral nutrition was started at a rate of $2 \mathrm{~mL} \cdot \mathrm{kg} \mathrm{bw}^{-1} \cdot \mathrm{h}^{-1}$ in addition to $500 \mathrm{~g}$ of their regular diet. The following 3 days, the diet was infused at a rate of $4 \mathrm{~mL} \cdot \mathrm{kg} \mathrm{bw}^{-1} \cdot \mathrm{h}^{-1}$ corresponding with $0.3 \mathrm{~g} \cdot \mathrm{kg} \mathrm{bw}^{-1} \cdot \mathrm{h}^{-1}$ of protein. The experimental design is depicted in (Figure 1).

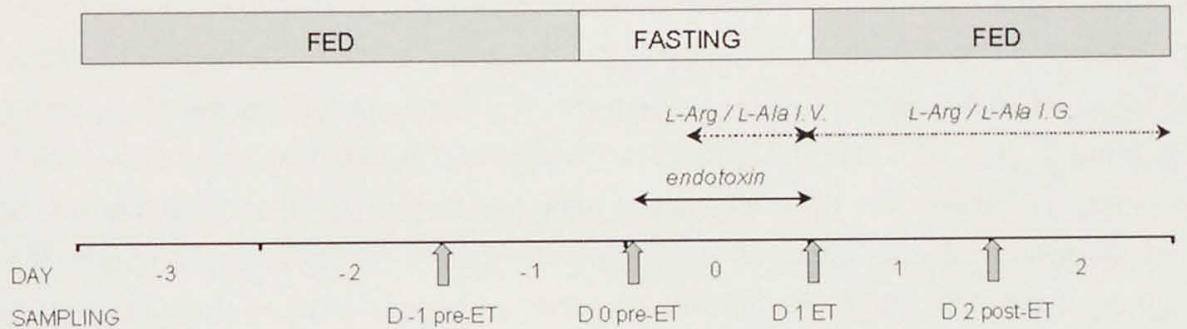

Figure 1. Illustration of the experimental design. I.V. = intravenously and I.G. = intragastrically.

The last day (d -1), experiments were started and control blood samples of the postprandial state were obtained from 14 pigs (Con-1). After an 8-h fast, control samples of the postabsorptive state were taken at $d 0$ (Con0). Directly afterward, an infusion of the lipopolysaccharide endotoxin from Escherichia coli (serotype 055:B5, Sigma Chemicals Co., St. Louis, MO, USA) dissolved in saline was started at a rate of $3 \mu \mathrm{g} \bullet \mathrm{kg}$ $\mathrm{bw}-1 \cdot \mathrm{h}-1$ via the venous (V2) catheter for a period of 24 hour. All pigs were administrated extra saline infusion to secure replenishment of intravascular volume losses; $30 \mathrm{ml} \bullet \mathrm{kg}$ $\mathrm{bw}-1 \cdot \mathrm{h}-1$ the first 8 hour and $20 \mathrm{ml} \cdot \mathrm{kg}$ bw- $1 \cdot \mathrm{h}-1$ the following 16 hour. Eight pigs were given an I.V. infusion of L-arginine (iso-osmolar, $5.3 \mu$ moløkg bw-1•min-1, pH 7.3) starting 8 hour after initiation of the endotoxin infusion. Six control pigs received alanine 
as a control amino acid at an isocaloric rate $(10.6 \mu \mathrm{mol} \bullet \mathrm{kg} \mathrm{bw}-1 \bullet \mathrm{min}-1, \mathrm{pH} 7.3)$ to check for eventual non-specific effects of giving an amino acid. The following day (d 1), at $24 \mathrm{~h}$ endotoxin infusion, blood was sampled from the L-arginine-treated group and the control group and the I.V. infusions of endotoxin, saline and amino acid were stopped. Immediately hereafter, enteral nutrition was restarted (4 mL・kg bw-1•h-1). The I.V. infusion of L-arginine or L-alanine was continued at an equal infusion rate as supplement in the intragastrically infused diet. The following day ( $\mathrm{d} \mathrm{2}$ ), $24 \mathrm{~h}$ after cessation of LPS, blood samples were obtained of the post-endotoxemic state from the L-arginine-treated (Arg2) and the control (Con2) pigs. During the experimental protocol, arterial blood pressure, weight and temperature of the pigs were monitored.

\section{Infusion protocol}

Before the start of the infusions, background blood samples were taken. On the morning of the trial, one hour before start of the endotoxin infusion, a primed infusion of paraaminohippuric acid (PAH, $25 \mathrm{mmol} / \mathrm{L}, \mathrm{A}$ 1422, Sigma Chemicals Co.) was conducted. Infusion was started at a rate of about $40 \mathrm{~mL} / \mathrm{h}$ per catheter through the $S$ and the $A 1$ catheter, after an initial bolus of $5 \mathrm{~mL}$ (31). Directly after the PAH bolus infusion, a primed and constant infusion of the stable isotopes $\mathrm{L}-\left[\right.$ ring $\left.-{ }^{2} \mathrm{H}_{5}\right]$ phenylalanine $\left(\mathrm{D}_{5}\right.$-phenylalanine; prime: $1 \mu \mathrm{mol} / \mathrm{kg}$ bw, infusion: $1.9 \mu$ moløkg bw $\left.{ }^{-1} \bullet \mathrm{h}^{-1}\right), \mathrm{L}-\left[\right.$ ring-3,5- $\left.{ }^{2} \mathrm{H}_{2}\right]$ tyrosine $\left(\mathrm{D}_{2}\right.$-tyrosine; prime: $1 \mu \mathrm{mol} / \mathrm{kg}$ bw, infusion: $\left.1.9 \mu \mathrm{mol} \bullet \mathrm{kg} \mathrm{bw}{ }^{-1} \bullet \mathrm{h}^{-1}\right)$ and of $\mathrm{L}-\left[1{ }^{13} \mathrm{C}\right]$ valine $\left({ }^{13} \mathrm{C}\right.$-valine;

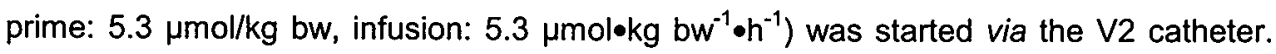
Also a primed and constant infusion of L-[guanidino- ${ }^{15} \mathrm{~N}_{2}$ ]arginine, L-[ureido- ${ }^{13} \mathrm{C} ; 5,5$ -

$\left.{ }^{2} \mathrm{H}_{2}\right]$ citrulline, $\left[{ }^{13} \mathrm{C}\right]$ urea and $\mathrm{D}-\left[6-{ }^{3} \mathrm{H}\right] \mathrm{glucose}$ was conducted, results of which will be reported elsewhere. Stable isotopes were purchased from Mass Trace, Woburn, MA, USA. During the last 60 min of the infusion protocol, an isotopic plateau (calculated slope of isotopic enrichment against time not different from zero) was observed (33). Also one hour after the start of the infusions, steady state conditions for PAH (data not shown) were obtained. Blood samples were collected in triplicate at 15-min intervals, during the last 60 min of the isotope and PAH infusion.

\section{Sample processing}

Promptly after sampling, blood was distributed in heparinized tubes (Sarstedt, Nümbrecht, Germany) on ice. For blood gas analysis (arterial $\mathrm{pH}, \mathrm{HCO}_{3}{ }^{-}, \mathrm{PO}_{\mathrm{O}}, \mathrm{PCO}_{\mathrm{CO}}$ and $\mathrm{S}_{\mathrm{O} 2}$ ), $0.2 \mathrm{~mL}$ blood was sealed airtight in heparinized $1 \mathrm{~mL}$ syringes and immediately analyzed on an automatic blood gas system (Acid Base Laboratory, Radiometer, Copenhagen, Denmark). Hematocrit was obtained with a microfuge. For determination of $\mathrm{PAH}$ concentration, $300 \mu \mathrm{L}$ of whole blood was added to $600 \mu \mathrm{L}$ of $120 \mathrm{~g} / \mathrm{L}$ trichloroacetic acid solution, thoroughly mixed, centrifuged and supernatant collected. 
Centrifugation was performed at $4{ }^{\circ} \mathrm{C}$ for $5 \mathrm{~min}$ at $8500 \mathrm{~g}$, plasma collected and kept on ice. For amino acid analysis, $500 \mu \mathrm{L}$ of plasma was deproteinized by mixing with $20 \mathrm{mg}$ dry sulfosalicylic acid. All samples were stored at $-80^{\circ} \mathrm{C}$ until further analysis.

\section{Biochemical analysis}

PAH was detected spectrophotometrically after deacetylation of the supernatant at $100^{\circ} \mathrm{C}$ for $45 \mathrm{~min}$ (13). Plasma concentrations of amino acids were determined by a fully automated High Performance Liquid Chromatography system (HPLC, Pharmacia, Woerden, The Netherlands) after precolumn derivatization with o-phthaldialdehyde (34). Amino acid enrichments of amino acids were calculated as tracer to tracee ratios (TTR) and were determined by a fully automated liquid chromatography-mass spectrometry system (LC-MS, Thermoquest LCQ, Veenendaal, the Netherlands) on-line connected to a HPLC (33).

Measurements of the acute-phase proteins haptoglobin, fibrinogen and $\alpha-1$ antitrypsin and total protein concentration were performed in plasma and carried out on a Nephelometer BN 100 (Dade Behring Vertriebs $\mathrm{GmbH}$ and Co., Germany). Proteins were determined using haptoglobin, fibrinogen and $\alpha-1$ antitrypsin anti-human antibodies from rabbits (Dade-Behring) as performed for human plasma samples. Standard curves for haptoglobin and fibrinogen were constructed using a purified human (Dade-Behring) and a secondary porcine standard (Sigma Chemicals Co.). High correlation coefficients $(>0.9)$ were found for the two proteins between the standards. Because a porcine standard for $\alpha-1$ antitrypsin was not available, protein concentrations were expressed in human units.

\section{Calculations}

The sum of AA represents the sum of measurable $\alpha$-amino acids (glutamine, glycine, threonine, histidine, citrulline, alanine, taurine, arginine, $\alpha$-amino butyric acid, tyrosine, valine, methionine, isoleucine, phenylalanine, tryptophan, leucine, ornithine and lysine) and BCAA the sum of branched-chain amino acids, valine, leucine and isoleucine.

Organ phenylalanine and valine kinetics

The portal-drained viscera are defined as the total of all portal-drained organs mainly representing the intestines. The splanchnic area is the sum of the portal-drained viscera and liver, therefore, calculations on liver were made by subtracting the portal-drained viscera from splanchnic values. Substrate metabolism across the hindquarter, the portal-drained viscera, the liver and the kidneys was calculated in a two-compartment model as described previously (38). The plasma flow rates $\left(\mathrm{mL} \cdot \mathrm{kg} \mathrm{bw}^{-1} \cdot \mathrm{min}^{-1}\right)$ across the organs were calculated using formula (1) based on the principle of indicator dilution methods in which I represents the rate of the 
PAH infused $\left(\mathrm{nmol} \cdot \mathrm{kg} \mathrm{bw}^{-1} \cdot \mathrm{min}^{-1}\right)$ and $[\mathrm{PAH}]_{\mathrm{v}}$ and $[\mathrm{PAH}]_{\mathrm{A}}$ the concentration $(\mu \mathrm{mol} / \mathrm{L})$ of $\mathrm{PAH}$ in the venous and arterial blood, respectively.

$$
\text { (1) } \text { FlOW }_{B L O O D}=1 /\left([\mathrm{PAH}]_{V}-[\mathrm{PAH}]_{\mathrm{A}}\right)
$$

The blood flow was converted to plasma flow by using the equation:

$$
\text { (2) Flow PLASMA }=\text { Flow }_{\text {BLOOD }} \times 100 /(100-\text { hematocrit) }
$$

Substrate net balance was defined as NB (nmol $\mathrm{kg} \mathrm{bw}^{-1} \cdot \mathrm{min}^{-1}$ ) and calculated in (3) by multiplying the mean plasma flow by the difference between $[\mathrm{V}]$ and $[\mathrm{A}]$ that represent the venous and arterial plasma concentration of the amino acid $(\mu \mathrm{mol} / \mathrm{L})$, respectively. Therefore, a positive NB represents net efflux and a negative NB represents net influx of substrate across the organ.

$$
\text { (3) } \quad \mathrm{NB}=\text { flow } \times([\mathrm{M}-[\mathrm{A}])
$$

The tracer net balance $\left(\mathrm{nb}, \mathrm{nmol} \cdot \mathrm{kg} \mathrm{bw}^{-1} \cdot \mathrm{min}^{-1}\right)$ was calculated in a similar way, using the $T T R_{A}$ and $T T R_{V}$ representing the arterial and venous tracer to tracee ratio of the amino acid, corrected for their background (pre-tracer infusion) TTR values:

(4) $n b=$ flow $\times\left\{\left([A] \times \operatorname{TTR}_{A}\right)-\left([V] \times T T R_{V}\right)\right\}$

The disposal rate $\left(\mathrm{nmol} \cdot \mathrm{kg} \mathrm{bw}^{-1} \cdot \mathrm{min}^{-1}\right)$ represents the total rate of metabolism of the amino acid (incorporation into protein plus degradation) and is calculated as follows: (5)

$$
\text { Disposal }=n b / T T R_{V}
$$

The TTR $v$ was thought to approach best the intracellular enrichment (precursor pool) of the organ (2). Since the NB of an amino acid across an organ is the net difference between production and disposal, the production is represented by:

(6) Production $=$ NB + Disposal

In the muscle, the gut and the kidneys, the disposal and production of phenylalanine are a reflection of protein synthesis and protein breakdown, respectively, as these organs have relatively low phenylalanine hydroxylase activity (32). Valine disposed in these organs can either become transaminated or be used in protein synthesis (26). In the liver, valine is mainly used for protein synthesis because valine degradation rate has been considered low $(16,26)$. Disposal of phenylalanine by the liver is a combination of protein synthesis and hydroxylation of phenylalanine to tyrosine (32). To calculate the organ rate of phenylalanine hydroxylation, conversion of $D_{5}$-phenylalanine into $D_{4}$-tyrosine $(\mathrm{PHE} \rightarrow$ TYR) is calculated using formula (10). The arterial $D_{5}$-phenylalanine enrichment was thought to best reflect the precursor pool. Because of eventual loss of tyrosine across the organ, the $\mathrm{nb}$ of $\mathrm{D}_{4}$-tyrosine ( $\mathrm{nb} \mathrm{D}_{\mathrm{A}-\mathrm{TYR}}$ ) was corrected in (9) for this fractional extraction (FE TYR). FE $E_{D 4-T Y R}$ was estimated by using the nb of $D_{2}$-tyrosine and calculated in (8): 
(8) $\quad F E_{\text {D4-TYR }}=\left\{\left([\mathrm{A}] \times T T R_{A}\right)-\left([\mathrm{V}] \times T T R_{V}\right)\right\}_{D 2-T T R} /\left([\mathrm{A}] \times T T R_{A}\right)_{D 2-T T R}$

$$
\mathrm{nb}_{\text {D4-TYR }}=\text { flow } \times\left\{\left([\mathrm{V}] \times \operatorname{TTR}_{V}\right)-\left([\mathrm{A}] \times \operatorname{TTR}_{\mathrm{A}}\right) \times(1-\mathrm{FE})\right\}_{\text {D4-TYR }}
$$

(10) Organ ${ }_{P H E} \rightarrow T Y R=n b_{D 4-T Y R} / T_{T R} R_{A D 5-P H E}$

Across the portal-drained viscera, the total production rate of a substrate measured is a combination of substrate derived from endogenous production plus output of substrate absorbed by the gut from the enterally infused meal that is not retained for disposal and escapes metabolism. This measured total production rate of a substrate by the portaldrained viscera can be corrected for the contribution of substrate that derives from nondisposed absorption. This gives a good approximate of the part of substrate production that represents endogenous production in the portal-drained viscera.

Whole body phenylalanine and valine kinetics The whole body rate of production or appearance ( $Q \mathrm{Ra}$; $\mathrm{nmol} \cdot \mathrm{kg} \mathrm{bw}^{-1} \cdot \mathrm{min}^{-1}$ ) of phenylalanine, valine or tyrosine is derived from formula (11) in which I represents the rate of $D_{5}$-phenylalanine, ${ }^{13} \mathrm{C}$-valine or $D_{4}$ tyrosine tracer infused $\left(\mathrm{nmol} \bullet \mathrm{kg} \mathrm{bw}^{-1} \cdot \mathrm{min}^{-1}\right)$. The TTR $\mathrm{T}_{\mathrm{A}}$ represents the isotopic TTR in the arterial plasma (38).

$$
\mathrm{QRa}=1 / T T R_{\mathrm{A}}
$$

The whole body rate of production of phenylalanine was used as an indication of whole body protein breakdown (Q PB) since this amino acid can not be newly synthesized. The rate of whole body hydroxylation of phenylalanine ( $Q$ PHE $\rightarrow$ TYR $)$ was represented by formula (12) in which the rate of $D_{5}$-phenylalanine to $D_{4}$-tyrosine conversion is calculated.

$$
Q_{\text {PHE } \rightarrow T Y R}=Q_{\text {Ra TYR }} \times T T R_{A \text { D4-TYR }} / T T R_{A D 5-P H E}
$$

$Q$ RA TYR is the whole body rate of appearance of tyrosine calculated from $D_{2}$-tyrosine isotope and TTR $R_{A}$ D-TYR and TTR A D5-PHE are the respectively TTR's of $D_{4}$-tyrosine and of $D_{5}$-phenylalanine in arterial plasma. The whole body rate of disappearance of $D_{5}$ phenylalanine is a combination of whole body protein synthesis rate (Q PS) and $Q$ PHE $\rightarrow$ TYR. Therefore, Q PS is represented as follows:

$$
Q P S=Q P B-Q_{P H E \rightarrow T Y R}
$$

\section{Statistics}

Results are presented as means \pm SEM. When the normality or equal variance test failed, data were transformed or log transformed where appropriate. The data were analyzed by a two-factor repeated measures ANOVA, using as within factor time with 4 levels (repeated measures: day $-1,0,1,2)$ and as between-factors treatment $(+/-\mathrm{L}$ arginine). Error alpha values are reported in tables in which $P_{T}$ indicates the significance of repeated measures over time, $P_{T \times G}$ the interaction of repeated measures and 
treatment group (time $x$ group) and $P_{G}$ the effect of L-arginine treatment. Levels of significance were set at $P<0.05$. When an overall significance for time was observed, univariate $F$ - tests were used to evaluate contrasts among the different days; to assess the effect of the short-term effect of endotoxin infusion plus fasting during fasting as compared to fasting state baseline values (Day 1 vs. 0) and the postponed effect of endotoxin infusion plus fasting during feeding as compared to fed baseline values (Day 2 vs. -1$)$. $P<0.05$ was considered significant. Contrasts between Day 0 and 1 were assessed for the arginine and alanine variables (concentration, net balance) within the Con and the Arg group, respectively after observing significant time $x$ group interaction as indicated in the text. In case overall significant time $x$ group interaction was observed, pair-wise group comparisons were performed at Day 1 and 2 using Bonferronni post-hoc tests to adjust the nominal level of significance for the multiple comparisons made $(P<0.05$ with correction).

Table 1. Organ plasma flow in pigs. Day -1: before endotoxin infusion in the fed state, Day 0 : in the fasting state, Day 1: during endotoxin infusion receiving L-alanine (Con) or L-arginine intravenously and Day 2: after endotoxin infusion receiving L-alanine or L-arginine intragastrically.

\begin{tabular}{|c|c|c|c|c|c|c|c|c|}
\hline & & \multirow{2}{*}{$\begin{array}{c}\text { Day -1 } \\
\text { FED }\end{array}$} & \multirow{2}{*}{$\begin{array}{c}\text { Day } 0 \\
\text { FASTING }\end{array}$} & \multirow{2}{*}{$\begin{array}{c}\text { Day } 1 \\
\text { FASTING }\end{array}$} & \multirow{2}{*}{$\begin{array}{l}\text { Day } 2 \\
\text { FED }\end{array}$} & \multicolumn{3}{|c|}{ ANOVA } \\
\hline & & & & & & PT & $P_{T \times G}$ & $P_{G}$ \\
\hline \multicolumn{9}{|c|}{$m L \cdot k g b w^{-1} \cdot \min ^{-1}$} \\
\hline \multirow[t]{2}{*}{ Portal-drained viscera } & Con & $40 \pm 3$ & $37 \pm 4$ & $39 \pm 4$ & $48 \pm 7$ & \multirow{2}{*}{0.03} & \multirow{2}{*}{0.95} & \multirow{2}{*}{0.50} \\
\hline & Arg & $35 \pm 2$ & $35 \pm 3$ & $42 \pm 6$ & $46 \pm 5$ & & & \\
\hline \multirow[t]{2}{*}{ Liver } & Con & $50 \pm 9$ & $59 \pm 7$ & $66 \pm 11$ & $55 \pm 20$ & \multirow{2}{*}{0.88} & \multirow{2}{*}{0.32} & \multirow{2}{*}{0.86} \\
\hline & Arg & $53 \pm 3$ & $69 \pm 4$ & $59 \pm 8$ & $54 \pm 7$ & & & \\
\hline \multirow[t]{2}{*}{ Hindquarter } & Con & $31 \pm 3$ & $31 \pm 5$ & $33 \pm 2$ & $33 \pm 7$ & \multirow{2}{*}{0.28} & \multirow{2}{*}{0.06} & \multirow{2}{*}{0.19} \\
\hline & Arg & $22 \pm 1$ & $32 \pm 4$ & $40 \pm 8$ & $41 \pm 8$ & & & \\
\hline \multirow[t]{2}{*}{ Kidneys } & Con & $38 \pm 6$ & $54 \pm 12$ & $68 \pm 16$ & $48 \pm 9$ & \multirow{2}{*}{0.42} & \multirow{2}{*}{0.78} & \multirow{2}{*}{0.12} \\
\hline & Arg & $30 \pm 2$ & $41 \pm 3$ & $45 \pm 11$ & $31 \pm 4$ & & & \\
\hline
\end{tabular}

Mean ( \pm SEM). Data were transformed or logtranformed as appropriate before analysis. Statistics by repeated measures ANOVA; $P$ values in table indicate significance for time $\left(P_{T}\right)$, for group $\left(P_{G}\right)$ and time $x$ group interactions $\left(P_{T \times G}\right)$. No significant group effects were observed. The significant time effect on portaldrained viscera flow showed no significant $(P>0.05)$ contrasts between Day 0and Day 1 and Day -1 and Day 2 (effect of the fasting endotoxin infusion period). Con group: $n=6$, Arg group: $n=8$.

\section{Results}

Organ plasma flow, temperature and blood gas values

Table 1 shows that the plasma flow across the hindquarter, the liver and the kidneys did not significantly change in time. Although overall time effect for the portal-drained viscera plasma flow was present, no significant effect of the fasting endotoxin infusion period was detected (Day 1 vs. 0 and Day 2 vs. -1 ). Organ plasma flows did not change upon L-arginine treatment. 
Body temperature of the pigs (Table 2) significantly changed over time; temperature increased as result of the fasting endotoxin infusion period (Day 1 vs. 0 ) and was still increased one day after cessation of the endotoxin infusion during intragastric feeding (Day 2 vs. -1). The arterial pH also increased with endotoxin infusion during fasting (Day 1 vs. 0). Arterial hematocrit levels, $\mathrm{HCO}_{3}{ }^{-}$and $\mathrm{PacO}_{2}$ dropped during the fasting endotoxin infusion period. Except for its lowering effect on $\mathrm{PacO}_{\mathrm{co}}$ did L-arginine intervention not exert any effects on temperature or blood gas values.

Acute-phase proteins The $\alpha-1$ antitrypsin and total protein concentration (Table 3) decreased as result of endotoxin infusion during fasting (Day 1 vs. 0 and Day 0 vs. 1). LArginine infusion had no influence on the measured acute-phase or total protein concentration.

Arterial concentrations As can be observed from Table 4, endotoxin infusion with prolonged fasting (Day 0 vs. 1) decreased the concentration of branched-chain amino acids (BCAA), Gly, GIn and Tyr but increased the concentration of Phe. Endotoxin infusion during fasting also decreased Arg concentrations within the control group and Ala concentrations within the Arg group from Day 0 to Day $1(P<0.01)$. L-Arginine and Lalanine intervention resulted in a rise in Arg and Ala concentration within the Arg and control group, respectively $(P<0.01)$. L-Arginine treatment time-dependently reduced the Gln and Tyr concentrations.

Table 2. Temperature, hematocrit and arterial blood gas values in pigs. Day -1: before endotoxin infusion in the fed state, Day 0: in the fasting state, Day 1: during endotoxin infusion receiving L-alanine (Con) or Larginine intravenously and Day 2: after endotoxin infusion receiving L-alanine or L-arginine intragastrically.

\begin{tabular}{|c|c|c|c|c|c|c|c|c|}
\hline & & \multirow{2}{*}{$\begin{array}{c}\text { Day -1 } \\
\text { FED }\end{array}$} & \multirow{2}{*}{$\begin{array}{c}\text { Day } 0 \\
\text { FASTING }\end{array}$} & \multirow{2}{*}{$\begin{array}{c}\text { Day } 1 \\
\text { FASTING }\end{array}$} & \multirow{2}{*}{$\begin{array}{l}\text { Day } 2 \\
\text { FED }\end{array}$} & \multicolumn{3}{|c|}{ ANOVA } \\
\hline & & & & & & $\mathrm{P}_{\mathrm{T}}$ & $P_{T x G}$ & $P_{G}$ \\
\hline \multirow[t]{2}{*}{ Temperature $\left({ }^{\circ} \mathrm{C}\right)$} & Con & $38.4 \pm 0.4$ & $38.1 \pm 0.4$ & $39.9 \pm 0.3$ & $38.6 \pm 0.1$ & \multirow{2}{*}{$0.001^{A B}$} & \multirow{2}{*}{0.82} & \multirow{2}{*}{0.51} \\
\hline & Arg & $38.2 \pm 0.4$ & $38.4 \pm 0.1$ & $39.6 \pm 0.4$ & $38.6 \pm 0.2$ & & & \\
\hline \multirow[t]{2}{*}{ Hematocrit } & Con & $27.5 \pm 0.8$ & $25.1 \pm 1.3$ & $23.1 \pm 1.5$ & $21.5 \pm 1.2$ & \multirow{2}{*}{$0.00^{A}$} & \multirow{2}{*}{0.47} & \multirow{2}{*}{0.38} \\
\hline & Arg & $29.8 \pm 0.4$ & $26.6 \pm 0.6$ & $22.9 \pm 0.8$ & $21.8 \pm 1.1$ & & & \\
\hline \multirow[t]{2}{*}{$\mathrm{pH}$} & Con & $7.4 \pm 0.0$ & $7.4 \pm 0.0$ & $7.5 \pm 0.0$ & $7.4 \pm 0.0$ & \multirow{2}{*}{$0.040^{A}$} & \multirow{2}{*}{0.74} & \multirow{2}{*}{0.73} \\
\hline & Arg & $7.4 \pm 0.0$ & $7.4 \pm 0.0$ & $7.4 \pm 0.0$ & $7.5 \pm 0.0$ & & & \\
\hline \multirow[t]{2}{*}{$\mathrm{Paco2}(\mathrm{mmol} / \mathrm{L})$} & Con & $6.6 \pm 0.3$ & $6.0 \pm 0.2$ & $5.4 \pm 0.2$ & $6.6 \pm 0.1$ & \multirow{2}{*}{$0.00^{A}$} & \multirow{2}{*}{0.17} & \multirow{2}{*}{0.03} \\
\hline & Arg & $6.1 \pm 0.1$ & $5.9 \pm 0.2$ & $4.6 \pm 0.2$ & $5.9 \pm 0.1$ & & & \\
\hline \multirow[t]{2}{*}{$\mathrm{Paoz}(\mathrm{mmol} / \mathrm{L})$} & Con & $12.7 \pm 0.7$ & $11.9 \pm 0.4$ & $12.3 \pm 0.2$ & $13.4 \pm 0.6$ & \multirow{2}{*}{0.08} & \multirow{2}{*}{0.86} & \multirow{2}{*}{0.70} \\
\hline & $\operatorname{Arg}$ & $12.9 \pm 0.2$ & $11.8 \pm 0.4$ & $13.1 \pm 0.5$ & $13.3 \pm 0.3$ & & & \\
\hline
\end{tabular}

Mean ( \pm SEM). Data were transformed or logtranformed as appropriate before analysis. Statistics by repeated measures ANOVA; $P$ values in table indicate significance for time $\left(P_{T}\right)$, for group $\left(P_{G}\right)$ and time $x$ group interactions $\left(P_{\text {TXG }}\right)$. ${ }^{A B} P<0.05$ by Univariate F-test; ${ }^{A}$ Day 1 vs. Day 0 and ${ }^{E}$ Day 2 vs. Day $-1=$ significant effect of the fasting endotoxin infusion period in, respectively, fasting and fed pigs compared to baseline values. Con group: $n=6$, Arg group: $n=8$. 
Table 3. The acute-phase proteins haptoglobin, fibrinogen, $\alpha-1$ antitrypsin and total protein in pigs. Day -1: before endotoxin infusion in the fed state, Day 0 : in the fasting state, Day 1: during endotoxin infusion receiving L-alanine (Con) or L-arginine intravenously and Day 2: after endotoxin infusion receiving L-alanine or L-arginine intragastrically.

\begin{tabular}{|c|c|c|c|c|c|c|c|c|}
\hline & & Day -1 & Day 0 & Day 1 & Day 2 & & ANOVA & \\
\hline & & FED & FASTING & FASTING & FED & PT & $P_{T \times G}$ & $\mathrm{PG}_{\mathrm{G}}$ \\
\hline \multicolumn{9}{|c|}{$g / L$} \\
\hline \multirow[t]{2}{*}{$\alpha-1$ Antitrypsin } & Con & $0.08 \pm 0$ & $0.08 \pm 0$ & $0.07 \pm 0.01$ & $0.06 \pm 0.01$ & \multirow{2}{*}{$0.002^{A B}$} & \multirow{2}{*}{0.77} & \multirow{2}{*}{0.40} \\
\hline & $\operatorname{Arg}$ & $0.08 \pm 0$ & $0.09 \pm 0$ & $0.08 \pm 0$ & $0.08 \pm 0.01$ & & & \\
\hline \multirow[t]{2}{*}{ Haptoglobin } & Con & $0.60 \pm 0.03$ & $0.59 \pm 0.03$ & $0.17 \pm 0.01$ & $0.17 \pm 0.01$ & \multirow{2}{*}{0.21} & \multirow{2}{*}{0.64} & \multirow{2}{*}{0.53} \\
\hline & Arg & $0.64 \pm 0.03$ & $0.61 \pm 0.03$ & $0.17 \pm 0.01$ & $0.18 \pm 0.01$ & & & \\
\hline \multirow[t]{2}{*}{ Fibrinogen } & Con & $0.40 \pm 1.14$ & $1.41 \pm 0.15$ & $1.67 \pm 0.23$ & $1.55 \pm 0.25$ & \multirow{2}{*}{0.37} & \multirow{2}{*}{0.26} & \multirow{2}{*}{0.20} \\
\hline & Arg & $1.26 \pm 0.90$ & $1.49 \pm 0.64$ & $1.09 \pm 0.15$ & $1.60 \pm 0.19$ & & & \\
\hline \multirow[t]{2}{*}{ Total protein } & Con & $30.6 \pm 1.4$ & $31.5 \pm 1.8$ & $26.0 \pm 1.4$ & $23.4 \pm 1.2$ & \multirow{2}{*}{$0.00^{A B}$} & \multirow{2}{*}{0.57} & \multirow{2}{*}{0.74} \\
\hline & Arg & $30.7 \pm 1.4$ & $32.6 \pm 1.4$ & $26.9 \pm 1.9$ & $25.5 \pm 1.8$ & & & \\
\hline
\end{tabular}

Mean ( \pm SEM). Data were transformed or logtranformed as appropriate before analysis. Statistics by repeated measures ANOVA; $P$ values in table indicate significance for time $\left(P_{\tau}\right)$, for group $\left(P_{G}\right)$ and time $x$ group interactions $\left(P_{T \times G}\right)$. ${ }^{A B} \mathrm{P}<0.05$ by Univariate F-test; ${ }^{A}$ Day 1 vs. Day 0 and ${ }^{8}$ Day 2 vs. Day $-1=$ significant effect of the fasting endotoxin infusion period in, respectively, fasting and fed pigs compared to baseline values. $P<0.05$ with correction by Bonferroni $t$-test: significant difference between groups.
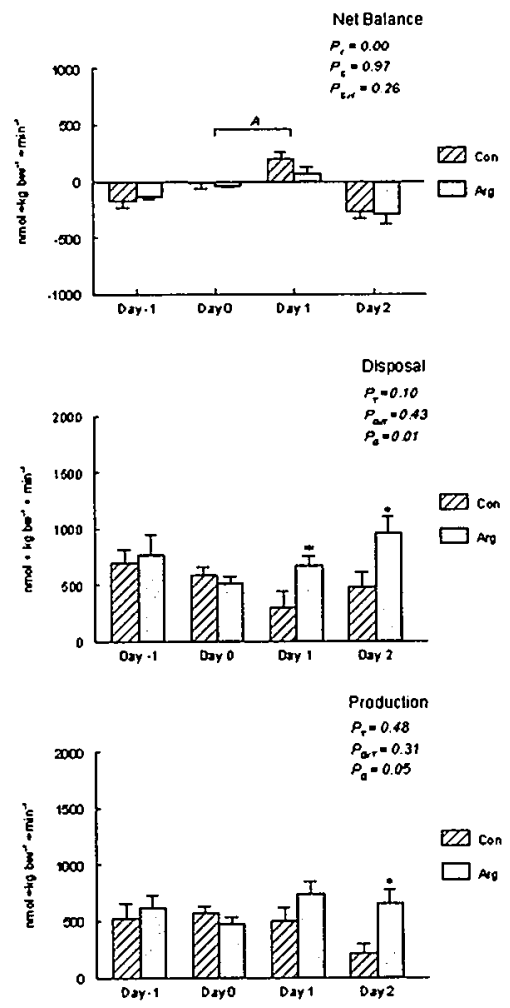

Figure 2. Rate of phenylalanine net balance, disposal (protein synthesis) and production (protein degradation) across the hindquarter in pigs before endotoxin infusion in the fed state (Day -1), in the fasting state (Day 0), during endotoxin infusion receiving $L$-alanine (Con) or $L$ arginine I.V. (Day 1) and after endotoxin infusion receiving L-alanine or L-arginine intragastrically (Day 2). Data are mean \pm SEM. Statistics by repeated measures ANOVA; $P$ values in figure indicate significance for time $\left(P_{T}\right)$, for group $\left(P_{G}\right)$ and time $x$ group interactions $\left(P_{T \times G}\right) .{ }^{A} P<0.05$ by Univariate F-test; Day 1 vs. Day $0=$ significant effect of the fasting endotoxin infusion period in fasting pigs compared to baseline values. No effect of the fasting endotoxin infusion period in fed pigs was observed (Day 2 vs. Day -1). ${ }^{*} P<0.05$ with correction by Bonferroni $t$-test: significant difference between treatment groups at Day 1 or Day 2. 


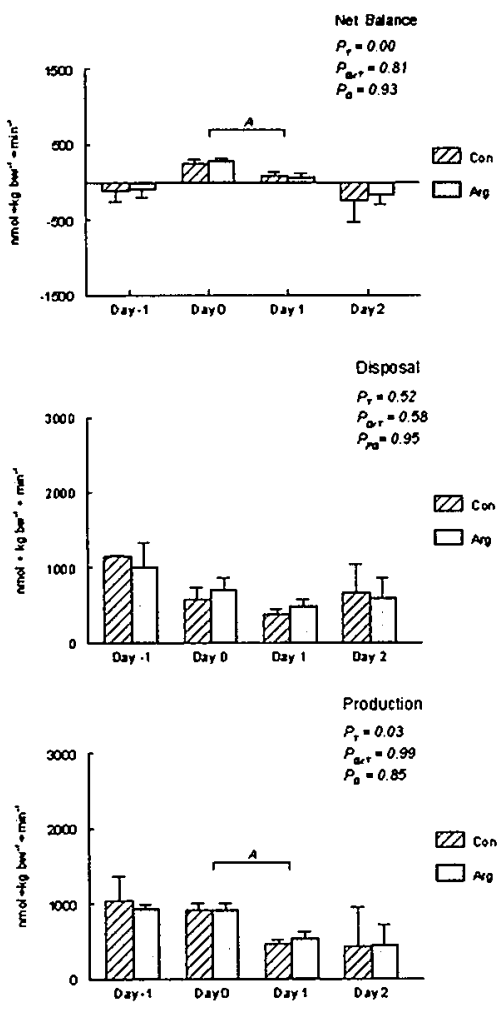

Figure 3. Rate of phenylalanine net balance, disposal (protein synthesis) and production (protein degradation) corrected for PHE production derived from enteral infusion across the portal-drained viscera in pigs before endotoxin infusion in the fed state (Day -1), in the fasting state (Day 0 ), during endotoxin infusion receiving L-alanine (Con) or L-arginine I.V. (Day 1) and after endotoxin infusion receiving $L$-alanine or L-arginine intragastrically (Day 2). Data are mean \pm SEM. Statistics by repeated measures ANOVA; $P$ values in figure indicate significance for time $\left(P_{T}\right)$, for group $\left(P_{G}\right)$ and time $x$ group interactions $\left(\mathrm{P}_{\mathrm{T} \times \mathrm{G}}\right) \cdot{ }^{A} \mathrm{P}<0.05$ by Univariate F-test; Day 1 vs. Day $0=$ significant effect of the fasting endotoxin infusion period in fasting pigs compared to baseline values. No effect of the fasting endotoxin infusion period in fed pigs was observed (Day 2 vs. Day -1). No significant group $x$ time or group interactions were observed.

Whole body rate of appearance After pigs were exposed to endotoxin infusion plus fasting at Day 1 , the whole body Phe appearance and Phe to Tyr conversion rates (Table 5) were elevated compared to Day 0 indicating accelerated protein breakdown and Phe hydroxylation. When feeding was resumed after cessation of endotoxin, increased protein breakdown and Phe hydroxylation were attenuated (Day 2 vs. -1). L-Arginine intervention time-dependently reduced the whole body protein degradation at Day 1 and Day 2 as measured from whole body Phe appearance. L-Arginine intervention also reduced the whole body Phe hydroxylation and protein synthesis although not timedependently. This implies that L-arginine exerts an inhibitory effect on the whole body protein turnover rate under endotoxemic conditions. The proportion Phe to Tyr hydroxylation of total protein breakdown amounted to $15-17 \%$ during endotoxin challenge in the fasted state and $10 \%$ after endotoxin challenge in the fed state. 
Table 4. Arterial concentrations in pigs. Day -1 : before endotoxin infusion in the fed state, Day 0 : in the fasting state, Day 1: during endotoxin infusion receiving L-alanine (Con) or L-arginine intravenously and Day 2: after endotoxin infusion receiving L-alanine or L-arginine intragastrically.

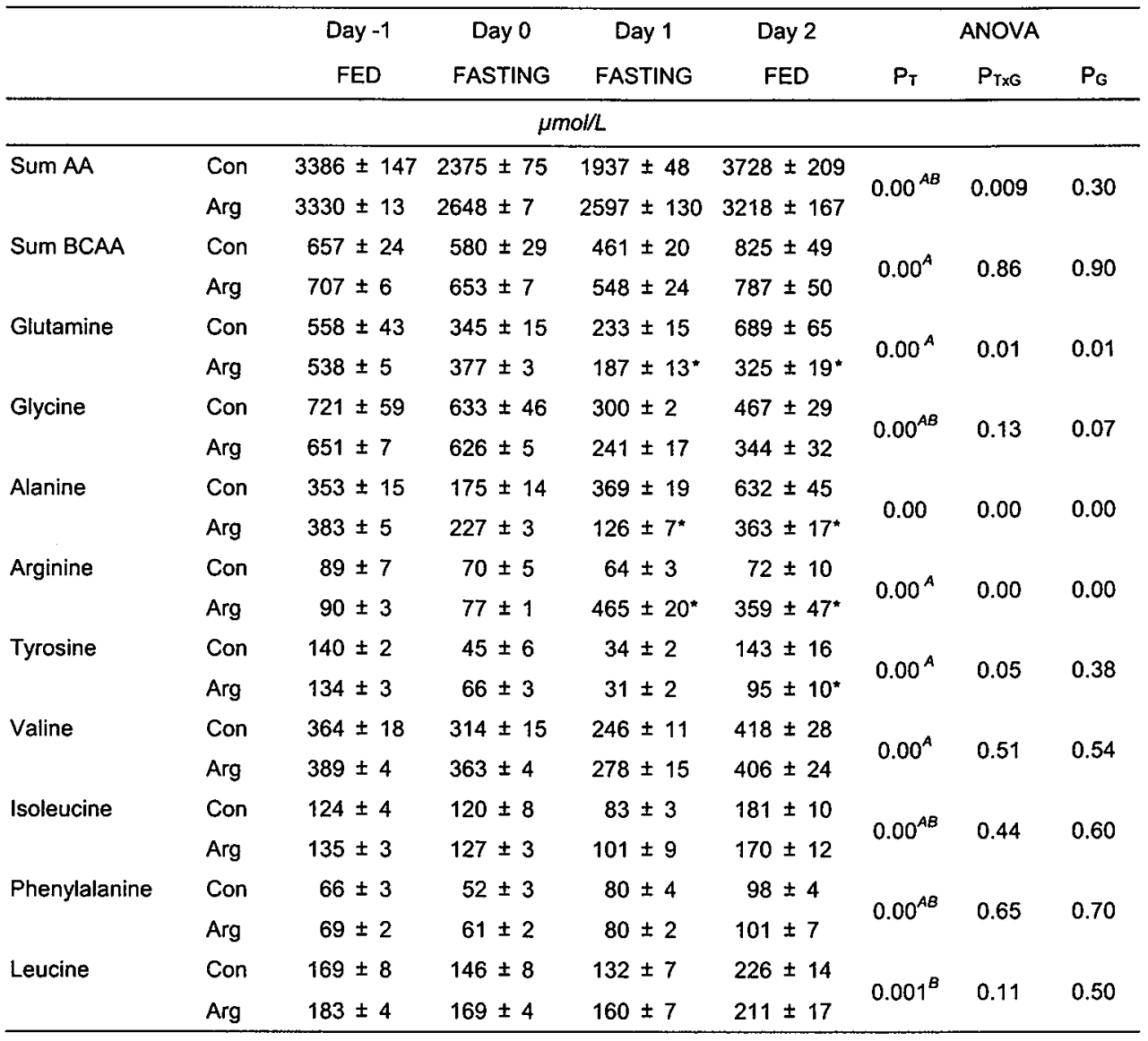

Abbreviations used: AA, sum measured amino acids; BCAA, sum branched-chain amino acids. Mean ( \pm $S E M)$. Data were transformed or logtranformed as appropriate before analysis. Statistics by repeated measures ANOVA; $P$ values in table indicate significance for time $\left(P_{T}\right)$, for group $\left(P_{G}\right)$ and time $x$ group interactions $\left(P_{T \times G}\right)$. ${ }^{A B} P<0.05$ by Univariate $F$-test; ${ }^{A}$ Day 1 vs. Day 0 and ${ }^{B}$ Day 2 vs. Day $-1=$ significant effect of the fasting endotoxin infusion period in, respectively, fasting and fed pigs compared to baseline values. ${ }^{*} P<0.05$ with correction by Bonferroni $t$-test: significant difference between treatment groups. Con group: $n=6$, Arg group: $n=8$. 
Table 5. Whole body appearance rate of Valine (Val), Tyrosine (Tyr) and Phenylalanine (Phe), Phe to Tyr conversion rate and difference between Phe appearance and conversion rate in pigs. Day -1: before endotoxin infusion in the fed state, Day 0 : in the fasting state. Day 1: during endotoxin infusion receiving Lalanine (Con) or L-arginine intravenously and Day 2: after endotoxin infusion receiving L-alanine or Larginine intragastrically.

\begin{tabular}{|c|c|c|c|c|c|c|c|c|c|}
\hline & & & \multirow{2}{*}{$\begin{array}{l}\text { Day }-1 \\
\text { FED }\end{array}$} & \multirow{2}{*}{$\begin{array}{c}\text { Day } 0 \\
\text { FASTING }\end{array}$} & \multirow{2}{*}{$\begin{array}{c}\text { Day } 1 \\
\text { FASTING }\end{array}$} & \multirow{2}{*}{$\begin{array}{c}\text { Day } 2 \\
\text { FED }\end{array}$} & \multicolumn{3}{|c|}{ ANOVA } \\
\hline & & & & & & & PT & $P_{T \times G}$ & $\mathrm{PG}_{\mathrm{G}}$ \\
\hline \multicolumn{10}{|c|}{ 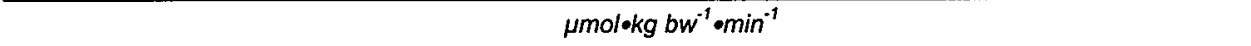 } \\
\hline \multirow[t]{2}{*}{ Val } & Q Ra & Con & $7.3 \pm 0.9$ & $5.7 \pm 0.6$ & $4.8 \pm 0.4$ & $7.3 \pm 0.7$ & \multirow{2}{*}{0.00} & \multirow{2}{*}{0.002} & \multirow{2}{*}{0.67} \\
\hline & & Arg & $6.5 \pm 0.9$ & $4.7 \pm 0.7$ & $3.5 \pm 0.3^{*}$ & $5.4 \pm 0.7^{\star}$ & & & \\
\hline \multirow[t]{2}{*}{ Tyr } & Q Ra & Con & $3.6 \pm 0.5$ & $1.8 \pm 0$ & $1.5 \pm 0.1$ & $3.0 \pm 0.4$ & \multirow{2}{*}{$0.00^{A}$} & \multirow{2}{*}{0.52} & \multirow{2}{*}{0.24} \\
\hline & & Arg & $3.0 \pm 0.1$ & $1.9 \pm 0$ & $1.2 \pm 0.1$ & $2.6 \pm 0.2$ & & & \\
\hline \multirow[t]{6}{*}{ Phe } & Q Ra & Con & $3.9 \pm 0.5$ & $2.4 \pm 0.1$ & $2.9 \pm 0.2$ & $5.1 \pm 0.3$ & \multirow{2}{*}{$0.00^{A B}$} & \multirow{2}{*}{0.02} & \multirow{2}{*}{0.005} \\
\hline & & $\operatorname{Arg}$ & $3.5 \pm 0.1$ & $2.4 \pm 0.1$ & $2.6 \pm 0.1$ & $4.0 \pm 0.2^{*}$ & & & \\
\hline & \multirow[t]{2}{*}{$Q_{\text {PHE } \rightarrow T Y R}$} & Con & $0.3 \pm 0$ & $0.2 \pm 0$ & $0.5 \pm 0.1$ & $0.5 \pm 0$ & \multirow{2}{*}{$0.002^{A B}$} & \multirow{2}{*}{0.22} & \multirow{2}{*}{0.02} \\
\hline & & Arg & $0.3 \pm 0$ & $0.3 \pm 0$ & $0.4 \pm 0$ & $0.4 \pm 0.1$ & & & \\
\hline & \multirow[t]{2}{*}{ (Q Ra) - (Q PHE $\rightarrow T Y R)$} & Con & $3.6 \pm 0.2$ & $2.2 \pm 0.1$ & $2.4 \pm 0.2$ & $4.9 \pm 0.3$ & \multirow{2}{*}{0.005} & \multirow{2}{*}{0.21} & \multirow{2}{*}{0.05} \\
\hline & & Arg & $3.2 \pm 0$ & $2.1 \pm 0$ & $2.2 \pm 0.1$ & $3.6 \pm 0.2$ & & & \\
\hline
\end{tabular}

Mean $( \pm S E M)$. Data were transformed or logtranformed as appropriate before analysis. ; $P$ values in table indicate significance for time $\left(P_{T}\right)$, for group $\left(P_{G}\right)$ and time $x$ group interactions $\left(P_{T \times G}\right)$. ${ }^{A B} P<0.05$ by Univariate F-test; ${ }^{A}$ Day 1 vs. Day 0 and ${ }^{B}$ Day 2 vs. Day $-1=$ significant effect of the fasting endotoxin infusion period in, respectively, fasting and fed pigs compared to baseline values. " $P<0.05$ with correction by Bonferroni $t$-test: significant difference between treatment groups. $Q$ Ra: whole body rate of Phe appearance representing protein degradation. $Q_{\text {PHE } \rightarrow \text { TYR }}$ : whole body Phe to Tyr conversion (hydroxylation) representing net protein balance. The difference between $Q$ Ra and $Q$ PHE $\rightarrow$ TYR represents protein synthesis.Con group: $n=6$, Arg group: $n=8$.

Hindquarter Fasting accompanied by endotoxin infusion increased the hindquarter efflux of total $A A$ and BCAA (Day 1 vs. 0). Fasting endotoxin infusion increased hindquarter Ala efflux within the Arg group and changed Arg influx into efflux within the control group (Table 6). L-Arginine significantly induced hindquarter influx of Arg. Figure 2 shows that fasting plus endotoxin infusion (Day 1 vs. 0 ) resulted in Phe efflux from the hindquarter, indicating net protein degradation. L-Arginine treatment in pigs increased Phe disposal as well as Phe production by the hindquarter (Figure 2) indicating that L-arginine stimulated both protein synthesis and degradation in the hindquarter area. The Phe net balance (net protein balance) across the hindquarter, nevertheless, remained unaffected with L-arginine. The stimulating effect of L-arginine on hindquarter protein turnover was not time-dependent, suggesting not being dependent on endotoxin infusion. The Phe hydroxylation rate in the hindquarter area (not shown) was not different from zero and not different between the two treatment groups, neither during, nor after endotoxin challenge. 
Table 6. Amino acid fluxes across the hindquarter in pigs. Day -1 : before endotoxin infusion in the fed state, Day 0: in the fasting state, Day 1: during endotoxin infusion receiving L-alanine (Con) or L-arginine intravenously and Day 2: after endotoxin infusion receiving L-alanine or L-arginine intragastrically.

\begin{tabular}{|c|c|c|c|c|c|c|c|c|c|}
\hline & & & \multirow{2}{*}{$\begin{array}{c}\text { Day }-1 \\
\text { FED }\end{array}$} & \multirow{2}{*}{$\begin{array}{c}\text { Day } 0 \\
\text { FASTING }\end{array}$} & \multirow{2}{*}{$\begin{array}{c}\text { Day } 1 \\
\text { FASTING }\end{array}$} & \multirow{2}{*}{$\begin{array}{l}\text { Day } 2 \\
\text { FED }\end{array}$} & \multicolumn{3}{|c|}{ ANOVA } \\
\hline & & & & & & & PT & $P_{T \times G}$ & $P_{G}$ \\
\hline \multicolumn{10}{|c|}{$\mu \mathrm{mol} \cdot \mathrm{kg} \mathrm{bw^{-1 }} \cdot \mathrm{min}^{-1}$} \\
\hline \multirow[t]{2}{*}{ Sum AA } & NB & Con & $-4.0 \pm 2.6$ & $1.9 \pm 1.4$ & $6.0 \pm 1.4$ & $-6.1 \pm 3.6$ & \multirow{2}{*}{$0.00^{A}$} & \multirow{2}{*}{0.46} & \multirow{2}{*}{0.49} \\
\hline & & Arg & $-3.5 \pm 3.0$ & $1.4 \pm 1.7$ & $7.8 \pm 4.1$ & $-6.6 \pm 3.0$ & & & \\
\hline \multirow[t]{2}{*}{ Sum BCAA } & NB & Con & $-1.6 \pm 0.9$ & $0.1 \pm 0.1$ & $0.9 \pm 0.3$ & $-1.7 \pm 0.7$ & \multirow{2}{*}{$0.009^{A}$} & \multirow{2}{*}{0.81} & \multirow{2}{*}{0.89} \\
\hline & & Arg & $-1.2 \pm 0.4$ & $0.2 \pm 0.1$ & $1.4 \pm 0.6$ & $-1.6 \pm 0.9$ & & & \\
\hline \multirow[t]{2}{*}{ Alanine } & NB & Con & $-0.6 \pm 0.3$ & $0.6 \pm 0.1$ & $1.3 \pm 0.4$ & $-1.6 \pm 0.6$ & \multirow{2}{*}{$0.00^{A}$} & \multirow{2}{*}{0.03} & \multirow{2}{*}{0.07} \\
\hline & & Arg & $-0.1 \pm 0.5$ & $0.4 \pm 0.2$ & $1.8 \pm 0.2$ & $-0.5 \pm 0.5$ & & & \\
\hline \multirow[t]{2}{*}{ Glutamine } & NB & Con & $0.8 \pm 0.4$ & $1.6 \pm 0.2$ & $1.4 \pm 0.2$ & $1.7 \pm 0.6$ & \multirow{2}{*}{0.51} & \multirow{2}{*}{0.14} & \multirow{2}{*}{0.68} \\
\hline & & Arg & $1.2 \pm 0.4$ & $1.1 \pm 0.3$ & $1.4 \pm 0.1$ & $1.7 \pm 0.4$ & & & \\
\hline \multicolumn{10}{|c|}{$\mathrm{nmol} / \mathrm{kg} \mathrm{bw}{ }^{-1} \cdot \min ^{-1}$} \\
\hline \multirow[t]{2}{*}{ Arginine } & NB & Con & $-445 \pm 68$ & $-149 \pm 53$ & $115 \pm 76$ & $-641 \pm 71$ & \multirow{2}{*}{0.00} & \multirow{2}{*}{0.26} & \multirow{2}{*}{0.05} \\
\hline & & $\operatorname{Arg}$ & $-476 \pm 115$ & $-230 \pm 57$ & $-50 \pm 112$ & $-1438 \pm 352$ & & & \\
\hline
\end{tabular}

Abbreviations used: AA, sum measured amino acids; BCAA, sum branched-chain amino acids; NB, net balance $(+=$ release). Mean $( \pm S E M)$. Data were transformed or logtranformed as appropriate before analysis. Statistics by repeated measures ANOVA; $P$ values in table indicate significance for time $\left(P_{T}\right)$, for group $\left(P_{G}\right)$ and time $x$ group interactions $\left(P_{\gamma \times G}\right)$. ${ }^{A} P<0.05$ by Univariate $F$-test; Day 1 vs. Day $0=$ significant effect of the fasting endotoxin infusion period in fasting pigs compared to baseline values. No effect of the fasting endotoxin infusion period in fed pigs was observed (Day 2 vs. Day -1).

Table 7. Amino acid fluxes corrected for the enteral infusion rate across the portal-drained viscera in pigs.

\begin{tabular}{|c|c|c|c|c|c|c|c|c|c|}
\hline & & & \multirow{2}{*}{$\begin{array}{c}\text { Day }-1 \\
\text { FED }\end{array}$} & \multirow{2}{*}{$\begin{array}{c}\text { Day } 0 \\
\text { FASTING }\end{array}$} & \multirow{2}{*}{$\begin{array}{c}\text { Day } 1 \\
\text { FASTING }\end{array}$} & \multirow{2}{*}{$\begin{array}{l}\text { Day } 2 \\
\text { FED }\end{array}$} & \multicolumn{3}{|c|}{ ANOVA } \\
\hline & & & & & & & $\mathrm{P}_{\mathrm{T}}$ & $P_{T \times G}$ & $P_{G}$ \\
\hline \multicolumn{10}{|c|}{$\mu \mathrm{mol} \cdot \mathrm{kg} \mathrm{bw}^{-1} \cdot \mathrm{min}^{-1}$} \\
\hline \multirow[t]{2}{*}{ Sum AA } & \multirow[t]{2}{*}{ NB } & Con & $21.2 \pm 5.0$ & $5.1 \pm 1.4$ & $1.5 \pm 1.1$ & $25.3 \pm 6.3$ & \multirow{2}{*}{0.01} & \multirow{2}{*}{0.84} & \multirow{2}{*}{0.94} \\
\hline & & Arg & $17.6 \pm 1.0$ & $6.7 \pm 3.0$ & $3.0 \pm 4.1$ & $23.1 \pm 4.2$ & & & \\
\hline \multirow[t]{2}{*}{ Sum BCAA } & \multirow[t]{2}{*}{ NB } & Con & $5.5 \pm 1.4$ & $1.5 \pm 0.6$ & $0.0 \pm 0.2$ & $5.6 \pm 1.9$ & \multirow{2}{*}{0.04} & \multirow{2}{*}{0.36} & \multirow{2}{*}{0.26} \\
\hline & & Arg & $4.6 \pm 0.7$ & $2.0 \pm 0.9$ & $0.5 \pm 1.1$ & $5.6 \pm 1.0$ & & & \\
\hline \multirow[t]{2}{*}{ Alanine } & \multirow[t]{2}{*}{ NB } & Con & $4.9 \pm 1.2$ & $1.0 \pm 0.2$ & $0.2 \pm 0.2$ & $11.1 \pm 2.0$ & \multirow{2}{*}{$0.00^{8}$} & \multirow{2}{*}{0.005} & \multirow{2}{*}{0.03} \\
\hline & & Arg & $4.5 \pm 0.5$ & $1.3 \pm 0.3$ & $1.1 \pm 0.2^{*}$ & $5.9 \pm 0.6^{*}$ & & & \\
\hline \multirow[t]{2}{*}{ Glutamine } & \multirow[t]{2}{*}{ NB } & Con & $-1.7 \pm 0.6$ & $-1.1 \pm 0.3$ & $-0.8 \pm 0.2$ & $-0.9 \pm 0.7$ & \multirow{2}{*}{$0.00^{A B}$} & \multirow{2}{*}{0.72} & \multirow{2}{*}{0.22} \\
\hline & & Arg & $-1.7 \pm 0.4$ & $-1.3 \pm 0.4$ & $0.0 \pm 0.3$ & $0.1 \pm 0.2$ & & & \\
\hline \multicolumn{10}{|c|}{$n m o l \cdot k g ~ b w^{-1} \cdot \min ^{-1}$} \\
\hline \multirow[t]{2}{*}{ Arginine } & \multirow[t]{2}{*}{ NB } & Con & $974 \pm 196$ & $292 \pm 76$ & $134 \pm 35$ & $161 \pm 170$ & \multirow{2}{*}{$0.00^{A B}$} & \multirow{2}{*}{0.00} & \multirow{2}{*}{0.05} \\
\hline & & Arg & $555 \pm 141$ & $295 \pm 74$ & $-613 \pm 276^{*}$ & $-590 \pm 545$ & & & \\
\hline
\end{tabular}

For explanations see Table $6 .{ }^{A B} \mathrm{P}<0.05$ by Univariate F-test; ${ }^{A}$ Day 1 vs. Day 0 and ${ }^{B}$ Day 2 vs. Day $-1=$ significant effect of the fasting endotoxin infusion period in, respectively, fasting and fed pigs compared to baseline values. ${ }^{*} P<0.05$ with correction by Bonferroni $t$-test: significant difference between treatment groups. Con group: $n=6$, Arg group: $n=8$. 
Table 8. Amino acid fluxes across the liver in pigs. Day -1 : before endotoxin infusion in the fed state, Day 0: in the fasting state, Day 1: during endotoxin infusion receiving L-alanine (Con) or L-arginine intravenously and Day 2: after endotoxin infusion receiving L-alanine or L-arginine intragastrically.

\begin{tabular}{|c|c|c|c|c|c|c|c|c|c|}
\hline & & & Day -1 & Day 0 & Day 1 & Day 2 & \multicolumn{3}{|c|}{ ANOVA } \\
\hline & & & FED & FASTING & FASTING & FED & $P_{T}$ & $P_{T \times G}$ & $P_{G}$ \\
\hline \multicolumn{10}{|c|}{ 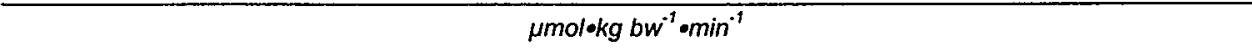 } \\
\hline \multirow[t]{2}{*}{ Sum AA } & NB & Con & $-9.1 \pm 5.2$ & $-4.1 \pm 1.8$ & $-24.2 \pm 2.2$ & $-21.8 \pm 4.5$ & \multirow{2}{*}{$0.00^{A}$} & \multirow{2}{*}{0.004} & \multirow{2}{*}{0.05} \\
\hline & & Arg & $-11.0 \pm 6.0$ & $-6.2 \pm 2.1$ & $-12.1 \pm 4.3$ & $-9.4 \pm 5.4$ & & & \\
\hline \multirow[t]{2}{*}{ Sum BCAA } & NB & Con & $-0.9 \pm 2.2$ & $-0.0 \pm 0.3$ & $1.3 \pm 1.1$ & $-3.0 \pm 1.7$ & \multirow{2}{*}{$0.00^{A B}$} & \multirow{2}{*}{0.30} & \multirow{2}{*}{0.52} \\
\hline & & Arg & $0.5 \pm 2.2$ & $0.4 \pm 0.2$ & $-1.6 \pm 0.4$ & $-3.6 \pm 0.8$ & & & \\
\hline \multirow[t]{2}{*}{ Alanine } & NB & Con & $-3.4 \pm 2.6$ & $-2.4 \pm 0.5$ & $-14.7 \pm 1.2$ & $-7.2 \pm 1.0$ & \multirow{2}{*}{$0.00^{A}$} & \multirow{2}{*}{0.00} & \multirow{2}{*}{0.002} \\
\hline & & Arg & $-2.5 \pm 1.0$ & $-3.4 \pm 0.5$ & $-5.0 \pm 0.5^{\star}$ & $-3.7 \pm 0.3^{*}$ & & & \\
\hline \multirow[t]{2}{*}{ Glutamine } & NB & Con & $-1.9 \pm 1.4$ & $-1.5 \pm 0.4$ & $-1.8 \pm 0.4$ & $-2.9 \pm 0.7$ & \multirow{2}{*}{0.11} & \multirow{2}{*}{0.15} & \multirow{2}{*}{0.02} \\
\hline & & Arg & $-1.9 \pm 0.7$ & $-1.3 \pm 0.4$ & $-1.4 \pm 0.3$ & $-2.4 \pm 0.4$ & & & \\
\hline \multicolumn{10}{|c|}{$n m o l \cdot k g ~ b w^{-1} \cdot \min ^{-1}$} \\
\hline \multirow[t]{2}{*}{ Arginine } & NB & Con & $149 \pm 432$ & $127 \pm 52$ & $-613 \pm 50$ & $-164 \pm 116$ & \multirow{2}{*}{$0.00^{A B}$} & \multirow{2}{*}{0.009} & \multirow{2}{*}{0.001} \\
\hline & & Arg & $-34 \pm 285$ & $-21 \pm 81$ & $-4205 \pm 484^{*}$ & $-1937 \pm 375^{*}$ & & & \\
\hline
\end{tabular}

Abbreviations used: AA, sum measured amino acids; BCAA, sum branched-chain amino acids; NB, net balance $(+=$ release). Mean $( \pm S E M)$. Data were transformed or logtranformed as appropriate before analysis. Statistics by repeated measures ANOVA; $P$ values in table indicate significance for time $\left(P_{T}\right)$, for group $\left(P_{G}\right)$ and time $x$ group interactions $\left(P_{T \times G}\right) .{ }^{A} P<0.05$ by Univariate $F$-test; Day 1 vs. Day $0=$ significant effect of the fasting endotoxin infusion period in fasting pigs compared to baseline values. No effect of the fasting endotoxin infusion period in fed pigs was observed (Day 2 vs. Day -1). ${ }^{*} P<0.05$ with correction by Bonferroni $t$-test: significant difference between treatment groups. Con group: $n=6$, Arg group: $n=8$.

Table 9. Amino acid fluxes across the kidneys in pigs.

\begin{tabular}{|c|c|c|c|c|c|c|c|c|c|}
\hline & & & \multirow{2}{*}{$\begin{array}{c}\text { Day }-1 \\
\text { FED }\end{array}$} & \multirow{2}{*}{$\begin{array}{c}\text { Day } 0 \\
\text { FASTING }\end{array}$} & \multirow{2}{*}{$\begin{array}{c}\text { Day } 1 \\
\text { FASTING }\end{array}$} & \multirow{2}{*}{$\begin{array}{c}\text { Day } 2 \\
\text { FED }\end{array}$} & \multicolumn{3}{|c|}{ ANOVA } \\
\hline & & & & & & & $P_{T}$ & $P_{T \times G}$ & $P_{G}$ \\
\hline \multicolumn{10}{|c|}{$\mu m o l \circ k g b w^{-1} \cdot \min ^{-1}$} \\
\hline \multirow[t]{2}{*}{ Sum AA } & NB & Con & $-1.8 \pm 4.0$ & $4.8 \pm 1.4$ & $2.6 \pm 4.8$ & $-8.3 \pm 7.6$ & \multirow{2}{*}{0.03} & \multirow{2}{*}{0.71} & \multirow{2}{*}{0.33} \\
\hline & & Arg & $-0.4 \pm 2.7$ & $6.4 \pm 3.2$ & $0.4 \pm 4.5$ & $-1.9 \pm 1.8$ & & & \\
\hline \multirow[t]{2}{*}{ Sum BCAA } & NB & Con & $0.6 \pm 0.8$ & $1.3 \pm 0.7$ & $1.1 \pm 1.0$ & $-0.8 \pm 2.1$ & \multirow{2}{*}{0.25} & \multirow{2}{*}{0.61} & \multirow{2}{*}{0.57} \\
\hline & & Arg & $0.0 \pm 0.8$ & $2.1 \pm 1.6$ & $1.4 \pm 1.2$ & $0.0 \pm 0.5$ & & & \\
\hline \multirow[t]{2}{*}{ Alanine } & NB & Con & $-0.6 \pm 0.4$ & $0.2 \pm 0.3$ & $3.1 \pm 1.0$ & $3.6 \pm 0.8$ & \multirow{2}{*}{$0.00^{8}$} & \multirow{2}{*}{0.009} & \multirow{2}{*}{0.02} \\
\hline & & Arg & $-0.1 \pm 0.7$ & $0.0 \pm 0.3$ & $0.5 \pm 0.6^{\star}$ & $-1.4 \pm 0.3^{*}$ & & & \\
\hline \multirow[t]{2}{*}{ Glutamine } & NB & Con & $0.6 \pm 0.2$ & $-0.1 \pm 0.3$ & $0.0 \pm 0.2$ & $0.0 \pm 0.7$ & \multirow{2}{*}{0.16} & \multirow{2}{*}{0.38} & \multirow{2}{*}{0.04} \\
\hline & & Arg & $1.0 \pm 0.2$ & $0.2 \pm 0.6$ & $-1.5 \pm 0.3$ & $-1.1 \pm 0.3$ & & & \\
\hline \multicolumn{10}{|c|}{$n m o l \circ k g ~ b w^{-1} \cdot \min ^{-1}$} \\
\hline \multirow[t]{2}{*}{ Arginine } & NB & Con & $356 \pm 170$ & $343 \pm 86$ & $179 \pm 34$ & $10 \pm 176$ & \multirow{2}{*}{$0.002^{A}$} & \multirow{2}{*}{0.001} & \multirow{2}{*}{0.07} \\
\hline & & Arg & $414 \pm 149$ & $472 \pm 99$ & $-532 \pm 113^{*}$ & $-507 \pm 142^{\star}$ & & & \\
\hline
\end{tabular}

For explanations see Table $8 .{ }^{A B} \mathrm{P}<0.05$ by Univariate F-test; ${ }^{A}$ Day 1 vs. Day 0 and ${ }^{B}$ Day 2 vs. Day $-1=$ significant effect of the fasting endotoxin infusion period in, respectively, fasting and fed pigs compared to baseline values. " $P<0.05$ with correction by Bonferroni $t$-test: significant difference between treatment groups. Con group: $n=6$, Arg group: $n=8$. 
Portal-drained viscera

In the L-arginine treatment group, influx of Arg across the portal-drained viscera (Table 7) was higher while in the L-alanine infused control group Ala efflux was lower. Figure 3 shows that fasting with endotoxin intervention reduced the net protein catabolism (Day 1 vs. 0). Addition with L-arginine neither affected the protein net balance, nor the protein synthesis or breakdown in the portal-drained viscera. The Phe hydroxylation rate across the portal-drained viscera during and after endotoxinchallenge was not significantly different from zero and not significantly different between the L-arginine-treated and control pigs (not shown).

Liver Compared to baseline values, the fasting with endotoxin challenge period resulted in net uptake of Val across the liver during as well after endotoxin infusion (Day 1 vs. 0 and Day 2 vs. -1 ), indicating endotoxin-induced net protein synthesis in the liver (Figure 4). L-Arginine treatment induced influx of Arg across the liver while, similarly, Lalanine infusion in the control group induced Ala influx across the liver (Table 8). LArginine infusion during and after endotoxin infusion decreased the Val disposal and production in the same extent (Figure 4) implying reduced liver protein turnover in absence of changes in liver protein net balance. The liver hydroxylation rate of Phe into Tyr (not shown) approximated $10 \%$ of the Phe disposal rate during fasting both before and after endotoxin treatment. During feeding. Phe hydroxylation nevertheless did not reach significance from zero due to relatively low Tyr enrichments in plasma.

Kidneys Within the control pigs, the Arg efflux from the kidneys decreased as consequence of fasting plus endotoxin challenge (Day 1 vs. $0, P<0.01$ ). Endotoxin infusion during fasting had no effect on the renal protein net balance, synthesis or breakdown since no significant effects on Phe disposal, production or net balance across the kidneys (Figure 5) were observed. Treatment with L-arginine induced Arg influx into the kidneys (Table 9) while L-alanine in the control group increased the Ala efflux. LArginine administration enhanced renal influx of glutamine although not time-dependent. L-Arginine also had no effect on the renal protein net balance, synthesis or breakdown (Figure 5). Moreover, the measured Phe to Tyr conversion across the kidneys (not shown) was not influenced by L-arginine treatment. Both during and after endotoxemia, irrespective of L-arginine infusion, the hydroxylation rate of Phe into Tyr amounted to $\sim 20 \%$ of the rate of Phe disposed. 
Figure 4. Rate of valine net balance, disposal (protein synthesis) and production (protein degradation) across the liver in pigs.
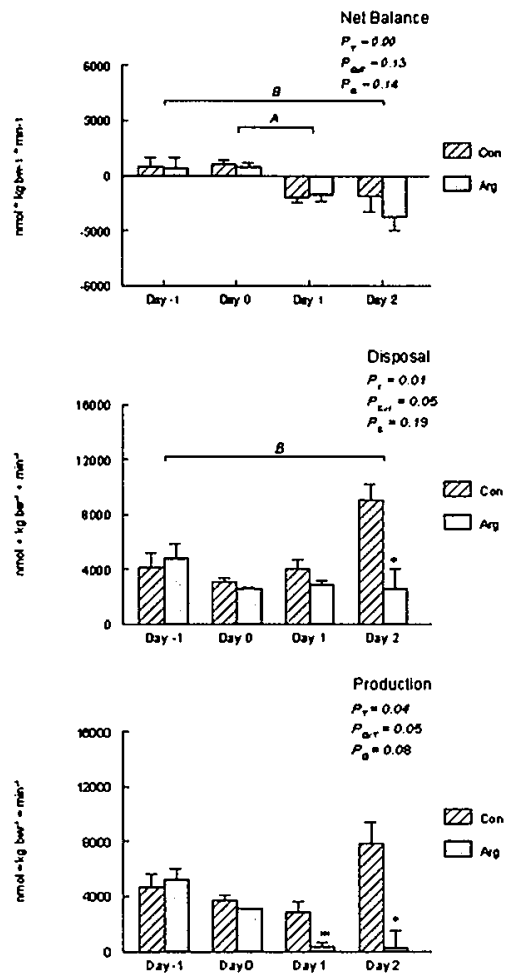

Figure 5. Rate of phenylalanine net balance, disposal (protein synthesis) and production (protein degradation) across the kidneys in pigs.

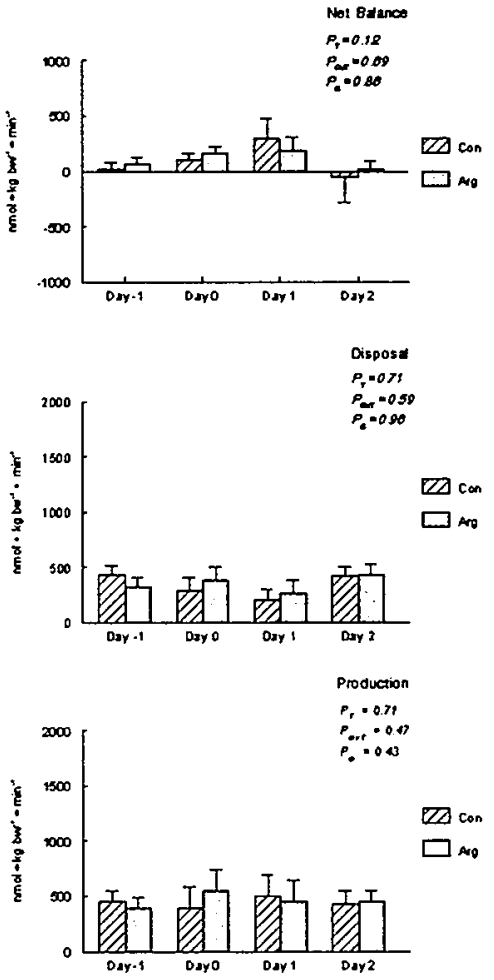

Before endotoxin infusion in the fed state (Day -1), in the fasting state (Day 0), during endotoxin infusion receiving L-alanine (Con) or L-arginine I.V. (Day 1) and after endotoxin infusion receiving L-alanine or Larginine intragastrically (Day 2 ). Data are mean \pm SEM. Statistics by repeated measures ANOVA; $P$ values in figure indicate significance for time $\left(P_{T}\right)$, for group $\left(P_{G}\right)$ and time $x$ group interactions $\left(P_{T \times G}\right){ }^{A 8} P<0.05$ by Univariate F-test; ${ }^{A}$ Day 1 vs. Day 0 and ${ }^{8}$ Day 2 vs. Day $-1=$ significant effect of the fasting endotoxin infusion period in, respectively, fasting and fed pigs compared to baseline values. ${ }^{\star} P<0.05$ with correction by Bonferroni $t$-test: significant difference between treatment groups at Day 1 or Day 2.

\section{Discussion}

Clinical trials using multiple component test-diets suggested anabolic and nitrogen retaining properties of L-arginine formulas (reviewed in: (17)). Intervention with L-arginine has shown to exert beneficial effects in a number of experimental diseases. However, only few studies have dealt with the sole effect of L-arginine on protein synthesis and degradation of the individual organs during stressed or infectious conditions. The current in vivo study was undertaken to investigate the influence of L-arginine on both protein synthesis and degradation under endotoxemic conditions. For this purpose, L-arginine was administered intravenously during endotoxin infusion and administered intragastrically as supplement in the diet after endotoxin infusion. We showed that under 
these endotoxemic conditions, L-arginine intervention increased protein turnover in hindquarter and decreased protein turnover in liver.

The current used model of endotoxemia was characterized by increased whole body and hindquarter protein breakdown as was measured by the stable phenylalanine isotope. In addition, tyrosine tracer revealed that the whole body phenylalanine hydroxylation simultaneously increased. The supplementation of L-arginine under endotoxemic conditions reduced whole body protein degradation and protein synthesis, i.e. turnover, and decreased whole body rate of phenylalanine hydroxylation.

Hindquarter The net protein breakdown that was observed across the hindquarter within the control group after endotoxin infusion reflects muscle catabolism, one of the main prominent features of the metabolic response to sepsis. Enteral nutrition one day after endotoxemia was capable of abolishing this net protein degradation. The intravenous L-arginine infusion during endotoxemia and intragastric L-arginine infusion one day after endotoxemia not only promoted protein synthesis in the hindquarter area but also increased the protein breakdown to a similar extent, therefore not resulting in improved net protein balance. In addition, the efflux of the total sum of amino acids by the hindquarter was not affected by L-arginine infusion, neither during nor after endotoxemia, implying unchanged net protein balance across hindquarter muscle. Enhanced muscle protein synthesis was also demonstrated in response to dietary Larginine in traumatized rats, nevertheless, in this study no concomitant muscle protein breakdown was measured (8). High protein turnover has been put forward to facilitate a rapid response by means of rapid alteration of enzyme concentrations and elimination of damaged proteins (25). Therefore, although no improved net protein balance was achieved across the muscle by L-arginine infusion, increased muscle protein turnover can be of benefit under altered physiological conditions such as septicemia.

Portal-drained viscera Only few flux studies with regard to the portal-drained viscera are available since the portal vein cannot easily be sampled. This study showed that endotoxin infusion during fasting reduced the portal-drained viscera protein net catabolism due to reduced protein degradation. Although we did not detect changes in phenylalanine disposal, a stimulation of intestinal protein synthesis was found in bacteremic rats (5). Previously it was shown that intravenous L-arginine supplementation in bowel-resected rats decreased the protein synthesis in the small intestine (37). In our model of endotoxemia, L-arginine supplementation exerted no effects on the portaldrained viscera rate of protein synthesis or breakdown neither during nor after endotoxemia. Because of a relatively high protein turnover rate of gut enterocytes, eventual effects of L-arginine on the protein metabolism of the portal-drained viscera may have become manifest at an earlier stage of endotoxemia. 
Liver During endotoxemia, cytokine-driven synthesis of acute-phase proteins by the liver is activated (23). The observed increase in net liver protein synthesis during and after endotoxin infusion, therefore, appears to represent enhanced acute-phase protein synthesis. The endotoxin-induced protein synthesis was however not reflected in increased plasma acute-phase protein concentrations. Plasma dilution resulting from fluid resuscitation during endotoxin infusion could have concealed possible increases in acute-phase protein concentrations. As far known, no studies on the effect of L-arginine on hepatic protein metabolism during sepsis or endotoxemia are available. In this in vivo model, L-arginine supplementation during and after endotoxin-infusion exerted a pronounced reducing effect on hepatic protein synthesis and breakdown, i.e., protein turnover, without affecting the protein net balance. The measured plasma acute-phase protein concentrations, nevertheless, did not decrease by L-arginine. Hence, whether the observed decrease in hepatic protein synthesis concerns delayed secretion of secretory proteins or rather concerns structural proteins of endogenous origin remains to be established. By the hepatic iNOS enzyme formed NO may be involved in the decreased hepatic protein turnover by means of inhibition of cell replication in the liver (10).

Phenylalanine hydroxylase was considered to be predominantly located in the liver (20, 32). Our data on measured phenylalanine to tyrosine conversion across the liver showed high variance due to low plasma tyrosine enrichments. Phenylalanine hydroxylation during fasting, irrespective of endotoxin or L-arginine infusion, accounted for approximately $10 \%$ of phenylalanine disposal in the liver, which approximates findings of a study performed on human liver in vivo (24).

Kidneys Dietary intervention with L-arginine has been associated with amelioration of a number of experimental kidney diseases (editorial: (29)). The effect of L-arginine administration on renal protein synthesis and degradation under septic conditions, however, remains to be explored. In our model of hyperdynamic endotoxemia with wellpreserved renal function, the renal protein synthesis and degradation remained unchanged by the treatment of L-arginine as measured by phenylalanine isotope. It thereby should to be taken into account that the kidneys of the pig appear to be capable of hydroxylating phenylalanine into tyrosine at a rate of $20 \%$ of the total phenylalanine disposal rate. This seems consistent with a study in which phenylalanine hydroxylation was found to occur at a rate of $40 \%$ in human kidney (24). Neither endotoxemia, nor Larginine exerted any effect on the rate of renal phenylalanine hydroxylation.

During L-arginine supplementation the decline in hepatic protein turnover surmounted the increase in muscle protein turnover, accounting for the decrease in whole body protein turnover at whole body level that was observed after endotoxemia. It is not excluded that differences in severity of the disease condition and related L-arginine requirements 
underlie the way by which L-arginine influences protein metabolism. Because in our sepsis model, L-arginine infusion increased the whole body production of NO (in press, Bruins et al, Crit Care Med, 2001), NO-mediated indirect effects on protein metabolism may not be excluded. L-Arginine as an intravenous or intragastric supplement may be considered promising with respect to its capacity to increase protein turnover in the hindquarter muscle after endotoxemia. Although no significant decrease in acute-phase protein concentration was observed, the in the liver effectuated decrease in protein turnover by L-arginine under (post)endotoxemic conditions may imply that L-arginine reduces the severity of the hepatic response to tissue injury and inflammation.

\section{Acknowledgments}

The authors wish to express many thanks to Mr. H.M.H. van Eijk, Mr. J.L.J.M. Scheijen and Mrs. K. Slot for measurements and Mrs. G.A.M. ten Have for assistance during operational and experimental procedures.

\section{References}

1. Beaumier, L., L. Castillo, Y. M. Yu, A. M. Ajami, and V. R. Young. Arginine: new and exciting developments for an "old" amino acid. Biomed Environmental Sci 9: 296-315, 1996.

2. Biolo, G., R. Y. D. Fleming, S. Maggi, and R. R. Wolfe. Transmembrane transport and intracellular kinetics of amino acids in human skeletal muscle. Am J Physiol 268: E75-E84, 1995.

3. Braga, M., L. Gianotti, A. Cestari, A. Vignali, F. Pellagatta, A. Dolci, and V. Di Carlo. Gut function and immune and inflammatory responses in patients perioperatively fed with supplemented enteral formulas. Arch Surg 131: 1257-1265, 1996.

4. Braga, M., L. Gianotti, G. Radaelli, A. Vignali, G. Mari, O. Gentilini, and V. Di Carlo. Perioperative immunonutrition in patients undergoing cancer surgery: results of a randomized double-blind phase 3 trial. Arch Surg 134: 428-33, 1999.

5. Breuille, D., M. Arnal, F. Rambourdin, G. Bayle, D. Levieux, and C. Obled. Sustained modifications of protein metabolism in various tissues in a rat model of long-lasting sepsis. Clin Sci (Colch) 94: 413-23, 1998.

6. Casey, L. C., R. A. Balk, and R. C. Bone. Plasma cytokine and endotoxin levels correlate with survival in patients with the sepsis syndrome [see comments]. Ann Intern Med 119: 771-8, 1993.
7. Chyun, J. H., and P. Griminger. Improvement of nitrogen retention by arginine and glycine supplementation and its relation to collagen synthesis in traumatized mature and aged rats. J Nutr 114: 1697-704, 1984.

8. Cui, X. L., M. Iwasa, Y. Iwasa, Y. Ohmori, A. Yamamoto, H. Maeda, M. Kume, S. Ogoshi, A. Yokoyama, T. Sugawara, and T. Funada. Effects of dietary arginine supplementation on protein turnover and tissue protein synthesis in scald-burn rats [In Process Citation]. Nutrition 15: 563-9, 1999.

9. Curran, R. D., T. R. Billiar, D. J. Stuehr, J. B. Ochoa, B. G. Harbrecht, S. G. Flint, and R. L. Simmons. Multiple cytokines are required to induce hepatocyte nitric oxide production and inhibit total protein synthesis. Ann Surg 212: 462-9; discussion 470-1, 1990.

10. Curran, R. D., F. K. Ferrari, P. H. Kispert, J. Stadler, D. J. Stuehr, R. L. Simmons, and T. R. Billiar. Nitric oxide and nitric oxide-generating compounds inhibit hepatocyte protein synthesis. Faseb J 5: 2085-92, 1991.

11. Daly, J. M., J. Reynolds, A. Thom, L. Kinsley, M. Dietrick-Gallagher, J. Shou, and B. Ruggieri. Immune and metabolic effects of arginine in the surgical patient. Ann Surg 208: 512-523, 1988.

12. De Bandt, J. P., and L. A. Cynober. Amino acids with anabolic properties. Curr Opin Clin Nutr Metab Care 1: 263-72, 1998. 
13. Deutz, N. E. P., R. PLM, B. MCF, v. B. CLH, and $P$. B. Soeters. Modification of the effects of blood on amino acid metabolism by intravenous isoleucine. Gastroenterology 101: 1613-1620, 1991.

14.Deutz, N. E. P., P. L. M. Reijven, G. Athanasas, and P. B. Soeters. Post-operative changes in hepatic, intestinal, splenic and muscle fluxes of amino acids and ammonia in pigs. Clin Sci 83: 607-614, 1992.

15. Doutheil, J., S. Althausen, $M$. Treiman, and $W$. Paschen. Effect of nitric oxide on endoplasmic reticulum calcium homeostasis, protein synthesis and energy metabolism [In Process Citation]. Cell Calcium 27: 107-15, 2000.

16. Elwyn, D. H. The role of the liver in regulation of amino acid and protein metabolism. In: Mammalian Protein Metabolism, edited by H. N. Munro. New York: Academic Press, 1970, p. 523-557.

17. Evoy, D., M. D. Lieberman, T. J. Fahey, 3rd, and J. M. Daly. Immunonutrition: the role of arginine. Nutrition 14: 611-7, 1998.

18. Freund, H., S. Atamian, J. Holroyde, and J. E. Fischer. Plasma amino acids as predictors of the severity and outcome of sepsis. Ann Surg 190: 571-6, 1979.

19. Hosomi, M., N. H. Stace, F. Lirussi, S. M. Smith, G. M. Murphy, and R. H. Dowling. Role of polyamines in intestinal adaptation in the rat. Eur J Clin Invest 17: 375-85, 1987.

20. Hsieh, M. C., and H. K. Berry. Distribution of phenylalanine hydroxylase (EC 1.14.3.1) in liver and kidney of vertebrates. J Exp Zool 208: 1617, 1979.

21. Hynninen, M., M. Valtonen, H. Markkanen, M. Vaara, P. Kuusela, I. Jousela, A. Piilonen, and O. Takkunen. Intramucosal $\mathrm{pH}$ and endotoxin and cytokine release in severe acute pancreatitis. Shock 13: 79-82, 2000.

22. Kolpakov, V., D. Gordon, and T. J. Kulik. Nitric oxide-generating compounds inhibit total protein and collagen synthesis in cultured vascular smooth muscle cells. Circ Res 76: 305-9, 1995.

23. Matuschak, G. M. Liver-lung interactions in critical illness. New Horiz 2: 488-504, 1994.

24. Moller, N., S. Meek, M. Bigelow, J. Andrews, and K. S. Nair. The kidney is an important site for in vivo phenylalanine-to-tyrosine conversion in adult humans: A metabolic role of the kidney. Proc Natl Acad Sci USA 97: 1242-6, 2000.

25. Newsholme, E. A., and A. R. Leech. Biochemistry for the medical sciences. New York: John Wiley \& Sons, 1983. p. 1-952.
26. Ooiwa, T., H. Goto, Y. Tsukamoto, T. Hayakawa, S. Sugiyama, N. Fujitsuka, and $Y$. Shimomura. Regulation of valine catabolism in canine tissues: tissue distributions of branchedchain aminotransferase and 2-oxo acid dehydrogenase complex, methacrylyl-CoA hydratase and 3-hydroxyisobutyryl-COA hydrolase. Biochim Biophys Acta 1243: 216$220,1995$.

27. Pond, $W$. Life cycle feeding: maternal nutrition and progeny development. In: Swine in biomedical research Plenum press, New York, 1986, p. 915-930.

28. Pui, $Y$. M., and $H$. Fisher. Factorial supplementation with arginine and glycine on nitrogen retention and body weight gain in the traumatized rat. J Nutr 109: 240-6, 1979.

29. Reyes, A. A., I. E. Karl, and S. Klahr. Role of arginine in health and in renal disease [editorial]. Am J Physiol 267: F331-46, 1994.

30. Saito, H., O. Trocki, S. L. Wang, S. J. Gonce, S. N. Joffe, and J. W. Alexander. Metabolic and immune effects of dietary arginine supplementation after burn. Arch Surg 122: 784-9, 1987.

31. Ten Have, G. A. M., M. C. F. Bost, J. C. A. W. Suyk-Wierts, A. E. J. M. van den Bogaart, and N. E. P. Deutz. Simultaneous measurement of metabolic flux in portally-drained viscera, liver, spleen, kidney and hindquarter in the conscious pig. Lab Anim 30: 347-358, 1996.

32. Tourian, A., J. Goddard, and T. T. Puck. Phenylalanine hydroxylase activity in mammalian cells. J Cell Physiol 73: 159-170, 1969.

33. Van Eijk, H. M. H., D. R. Rooyakkers, and N. E. $P$. Deutz. Determination of amino acid isotope enrichment using liquid chromatography-mass spectrometry. Anal Biochem 271: 8-17, 1999.

34. Van Eijk, H. M. H., D. R. Rooyakkers, and N. E. $P$. Deutz. Rapid routine determination of amino acids in plasma by high-performance liquid chromatography with a 2-3 $\mu \mathrm{M}$ Spherisorb ODS II column. J Chromatogr 620: 143-148, 1993.

35. Visek, W. J. Arginine and disease states. J Nutr 115: 532-41, 1985.

36. Weimann, A., L. Bastian, W. E. Bischoff, M. Grotz, M. Hansel, J. Lotz, C. Trautwein, G. Tusch, H. J. Schlitt, and G. Regel. Influence of arginine, omega- 3 fatty acids and nucleotidesupplemented enteral support on systemic inflammatory response syndrome and multiple organ failure in patients after severe trauma. Nutrition 14: 165-72, 1998. 
37. Welters, C. F., C. H. Dejong, N. E. Deutz, and E. Heineman. Effects of parenteral arginine supplementation on the intestinal adaptive response after massive small bowel resection in the Rat. J Surg Res 85: 259-66, 1999.
38. Wolfe, R. R. Radioactive and stable isotope tracers in biomedicine. Principles and practice of kinetic analysis. New York: Wiley-Liss, 1992. 
IN VIVO MEASUREMENTS OF ORGAN NITRIC OXIDE PRODUCTION DURING HYPERDYNAMIC ENDOTOXEMIA IN PIGS DETERMINED FROM STABLE ISOTOPE CONVERSION

\author{
Maaike J Bruins \\ Wouter H Lamers * \\ Peter B Soeters \\ Alfred J Meijer * \\ Nicolaas EP Deutz
}

Department of Surgery, Maastricht University, The Netherlands

"Departments of Anatomy \& Embryology (WHL) and Biochemistry (AJM), University of Amsterdam, AMC, The Netherlands 


\section{Abstract}

Inducible nitric oxide synthase (iNOS) activity depends on extracellular arginine, availability of which may become limiting as substrate for nitric oxide (NO) production under sustained conditions of sepsis. The quantitative relation between organ arginine utilization or production and NO metabolism in vivo was established as a reference for pharmaceutically modulating organ NO production.

Pigs (control, $n=7$, endotoxin, $n=7$ ) were instrumented with catheters to enable NO measurements across the hindquarter $(\approx$ muscle), the portal-drained viscera ( $\approx$ gut) and the liver. As a model of sepsis, pigs received a continuous i.v. endotoxin infusion and saline resuscitation. After an initial prime, the stable isotope ${ }^{15} \mathrm{~N}_{2}$-arginine and the flow indicator P-aminohippurate were infused one hour prior to blood sampling. Organ NO synthesis rate was estimated from ${ }^{15} \mathrm{~N}_{2}$-arginine to ${ }^{15} \mathrm{~N}$-citrulline conversion and plasma flow. Blood was obtained before and 24 hour after endotoxin or saline infusion.

During endotoxin infusion a hyperdynamic circulation was achieved, characteristic of the human septic condition.

Endotoxin induced NO production by the gut and liver, which was quantitatively matched by enhanced arginine disposal.

In the hindquarter, the arginine production (mainly from protein degradation) was accelerated during endotoxemia providing two-third of the arginine appearing in the circulation. Nevertheless, arginine plasma concentration was lower in endotoxin-treated animals than in controls.

On a whole-body level, the muscle serves as main arginine supplier and, in view of the reduced arginine plasma levels, seems critical in providing arginine as the precursor for NO to the splanchnic region.

\section{Introduction}

The role of nitric oxide (NO) in sepsis represents one of the most studied yet controversial subjects. NO has a dual role inasmuch as its biological effect differs depending on its quantities produced $(19,28)$. Generated in physiologic amounts by the constitutive isoform of the NO synthase (CNOS), NO has clearly been shown to possess an indispensable role in organ perfusion by acting as a regulator of vascular tone and blood flow distribution. In basic amounts, NO is also considered to represent an important mediator in immune-mediated disease by protecting against inflammatory injury in various tissues. On the other hand, formed in high quantities by the inducible 
NOS type (iNOS), NO is considered harmful since at higher concentrations, NO may have pro-inflammatory effects. Moreover, high NO production rate may exert a detrimental effect by contributing to hypotension via cardiodepression and vascular hyporeactivity (23).

Inhibitors of NOS were proposed as a therapeutic modality to compensate these complications by reducing hyperproduction of NO in sepsis. However, thus far both nonselective and selective inhibitors showed equivocal effects since their effects were strongly related to the experimental model of sepsis $(23,24)$. Thus, although overproduction of NO may have harmful effects, a basic level of NO production seems to be required for the maintenance of organ function in sepsis since inhibition of production of NO may threaten the protective role of controlled NO production in inflammatory disease. Under immune-mediated conditions of high NO output such as sepsis, the availability of arginine from blood is of importance for the biosynthesis of NO because cells expressing the iNOS isoform are strictly dependent on extracellular arginine and arginine uptake may become rate-limiting for NO synthesis by these cells $(14,32,35)$. Several studies have shown decreased arterial arginine levels under endotoxemic conditions $(9,25)$. Therefore, as a result of decreased local availability for its semiessential substrate arginine, the protective role of NO in gut and liver may become compromised at a time of increased demand under conditions of sustained stress in long-term sepsis.

In view of the fact that NO plays a paradoxical role in inflammation and blood flow regulation, development of drugs that modulate the NO production is extremely difficult. Supplying arginine as the precursor of $\mathrm{NO}$ and reducing NO production by NOS inhibitors have both been suggested as a therapeutic modality in disease, including sepsis. However, to be able to manipulate NO production rates meaningfully, it is necessary to quantify arginine and NO metabolism in the different organs during sepsis. For that reason, we conducted a study in pigs, measuring arginine and NO metabolism in the gut, liver and hindquarter during endotoxemia, using the stable isotope L-[guanidino${ }^{15} \mathrm{~N}_{2}$ ]arginine and its conversion to $\mathrm{L}-\left[\right.$ ureido- $\left.{ }^{15} \mathrm{~N}\right]$ citrulline. The pig was chosen as a chronically instrumented large animal model, because organ physiology and metabolic changes in this animal are in many respects comparable with those observed in humans $(13,26)$. 


\section{Methods}

\section{Surgical procedure}

The study was approved by the Animal Ethics Committee of the Maastricht University. Fourteen female crossbred pigs (Yorkshire $\times$ Dutch Landrace: weighting 20-22 kg) received water ad libitum and $1 \mathrm{~kg}$ of regular pig feed (Landbouwbelang, Roermond, the Netherlands; $16 \%$ crude protein) daily in the morning, supporting a growth rate of approximately $300 \mathrm{gram}$ per day. After an overnight fast, all animals were anesthetized with a mixture of $\mathrm{N}_{2} \mathrm{O} / \mathrm{O}_{2}(1: 2)$ and halothane $(0.8 \%)$ and intubated. After intubation, the pigs were intravenously administrated $6.25 \mathrm{mg} / \mathrm{kg}$ lincomycin.2HCl (Lincomycin, A.U.V., Cuyk, the Netherlands) as bactericidal prophylaxis and $12.5 \mathrm{mg} / \mathrm{kg}$ spectinomycin. $\mathrm{HCl}$ lyophil (Spectinomycin, A.U.V.) as bacteriostatic prophylaxis. To avoid coagulation and as postoperative analgesia, $50 \mathrm{mg} / \mathrm{kg}$ flunixine (Finadyne, Schering-Ploegh, Brussels, Belgium) was given. The surgical procedure has been described in detail (10). In brief, after a midline laparotomy was performed, seven catheters were inserted in the blood vessels as depicted in Figure 1. Two catheters were placed in the abdominal aorta; just above the bifurcation (A1) and just above the right renal vein (A2), and two in the inferior caval vein at the corresponding positions ( $V 1$ and $V 2$, respectively). Furthermore, catheters were placed in the portal $(P)$, hepatic $(H)$ and splenic $(S)$ veins.

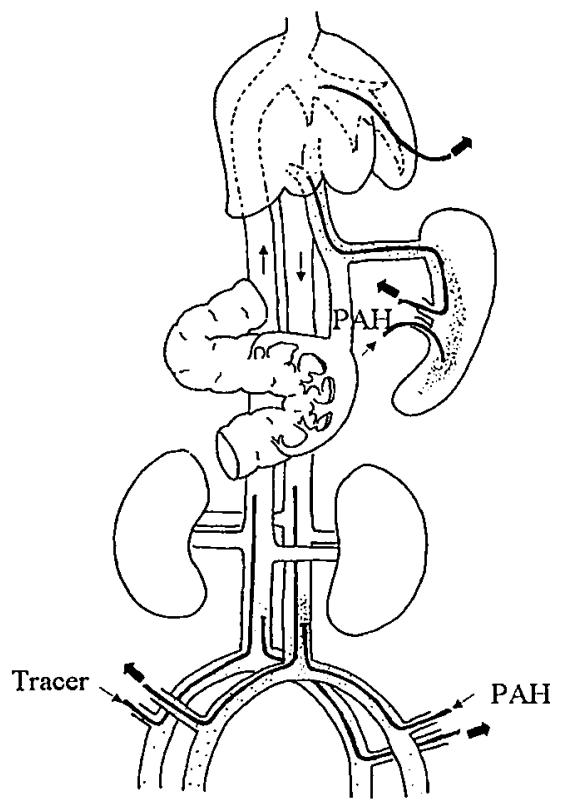

Figure 1. Schematic illustration of the catheter implantation. Catheters were placed in the abdominal aorta just above the bifurcation (A1) and just above the right renal vein (A2), in the inferior caval vein at the corresponding positions ( $V 1$ and $V 2$, respectively) and in the portal $(P)$, hepatic $(H)$ and splenic $(S)$ vein. The $A 1$ and $S$ catheters were used for the infusion of $P$ aminohippurate whereas the V2 catheter was used for isotope and endotoxin infusion. The $A 2, P, H$ and $V 1$ catheters were used for blood sampling. 
The abdominal aorta (A1) and the splenic vein catheters were used for the infusion of $P$ aminohippurate (PAH). The caval vein (V2) catheter was used for isotope and endotoxin infusion. The abdominal aorta (A2), portal (P), hepatic and caval vein (V1) catheters were used for blood sampling. Also, a gastrostomy catheter was inserted. All catheters were tunneled through the abdominal wall and skin. Catheters were flushed with heparinized saline $(50 \mathrm{U} / \mathrm{mL})$ to maintain patency, as previously described (31). Postoperative care was standardized (37). Each pig wore a canvas harness to allow easy handling of the animal. Ten days after surgery when pigs were fully recovered without signs of infection, liquid enteral nutrition (Nutrison Steriflo High-Protein, Nutricia, Zoetermeer, the Netherlands) was infused into the gastrostomy catheter via a swivel system connected to a pump for a period of 4 days to standardize daily food intake. During blood sampling, pigs were held in a small cage to which they were accustomed.

\section{Experimental protocol}

The night prior the endotoxin infusion, enteral nutrition was stopped. The next morning, the pigs were placed in a movable cage and experiments were conducted. Seven pigs received $3 \mu \mathrm{g} \bullet \mathrm{kg}^{-1} \bullet \mathrm{h}^{-1}$ endotoxin (Escherichia coli lipopolysaccharide 055:B5, Sigma Chemicals Co., St. Louis, MO, USA) in saline for $24 \mathrm{~h}$ via the caval vein (V2) catheter. Seven control animals received saline at an equivalent infusion rate. Also via this catheter, all pigs received extra saline infusion: $30 \mathrm{~mL} \cdot \mathrm{kg}^{-1} \cdot \mathrm{h}^{-1}$ during the first $8 \mathrm{~h}$ and 20 $\mathrm{mL} \cdot \mathrm{kg}^{-1} \cdot \mathrm{h}^{-1}$ the following $16 \mathrm{~h}$ to ensure the development of a hyperdynamic septic condition. Samples were taken before (CON 0 group) and $24 \mathrm{~h}$ after the start of endotoxin (ET 24 group) or saline (CON 24 group) infusion. Pigs were fasted during the whole period of endotoxin infusion. Also, rectal temperature, mean arterial pressure (MAP) and heart rate (HR) were regularly monitored in both groups. The endotoxemia model has been validated in a different study in which cardiac output was followed during 24-h endotoxin infusion.

\section{Infusion protocol}

One hour before blood sampling, P-aminohippurate (PAH, $25 \mathrm{mM}, \mathrm{A}$ 1422, Sigma Chemicals Co., St. Louis, MO, USA) and isotope primed-constant-infusion protocois were performed. PAH was infused at a rate of $40 \mathrm{~mL} / \mathrm{h}$ per catheter through the splenic vein and the abdominal aorta (A1) catheters after an initial prime of $5 \mathrm{~mL}(37)$. Immediately thereafter, a priming dose $(0.1 \mu \mathrm{mol} / \mathrm{kg})$ followed by a constant infusion $(0.1$ $\mu$ mol $\left.\bullet \mathrm{kg}^{-1} \cdot \mathrm{h}^{-1}\right)$ of $\mathrm{L}$-[guanidino- ${ }^{15} \mathrm{~N}_{2}$ ] arginine $\left({ }^{15} \mathrm{~N}_{2}\right.$-arginine, Mass Trace, Woburn, MA, USA) was given via the caval vein (V2) catheter. Before the start of the infusions, background blood samples were collected. One hour after the start of the infusions, 
steady state conditions for PAH (37) and ${ }^{15} \mathrm{~N}_{2}$-arginine (38) were obtained and blood was sampled.

\section{Sample processing}

Promptly after sampling, blood was distributed in heparinized tubes (Sarstedt, Nümbrecht, Germany) on ice. Hematocrit was determined to enable calculation of plasma flow from blood flow. For arterial blood gas analysis $\left(\mathrm{pH}\right.$, bicarbonate, $\mathrm{PaO}_{2}$ and $\mathrm{PaCO}_{2}$ ), $200 \mu \mathrm{L}$ blood was sealed airtight in heparinized $1 \mathrm{~mL}$ syringes and immediately analyzed on an automatic blood gas system (Acid Base Laboratory, Radiometer, Copenhagen, Denmark). Centrifugation was performed at $4^{\circ} \mathrm{C}$ for $5 \mathrm{~min}$ at $8500 \mathrm{~g}$, plasma collected and kept on ice. For urea determinations $900 \mu \mathrm{L}$ plasma was added to $90 \mu \mathrm{L} 50 \%$ trichloroacetic acid solution, ensuring stability of the substances. To determine plasma nitrite $\left(\mathrm{NO}_{2}{ }^{\circ}\right)$ and nitrate $\left(\mathrm{NO}_{3}{ }^{\circ}\right)$ concentrations, $100 \mu$ plasma was deproteinized with $200 \mu \mathrm{l}$ acetonitrile. For amino acid analysis, $500 \mu \mathrm{L}$ of plasma was deproteinized by mixing with $20 \mathrm{mg}$ dry sulfosalicylic acid. For PAH determination, $300 \mu \mathrm{l}$ of whole blood was added to $600 \mu \mathrm{L}$ of $12 \%$ (w/v) trichloroacetic acid solution, thoroughly mixed, centrifuged and supernatant collected. All samples were frozen in liquid nitrogen and stored at $-80^{\circ} \mathrm{C}$.

\section{Biochemical analysis}

Whole blood PAH concentration and plasma urea concentration were detected spectrophotometrically by standard enzymatic methods on an automated analysis system (Cobas Mira-S, Hoffmann-La Roche, Basel, Switzerland). PAH was detected after deacetylation of the supernatant at $100^{\circ} \mathrm{C}$ for $45 \mathrm{~min}$ (37). Before urea measurements, plasma ammonia was removed by conversion into glutamate. Urea was enzymatically converted into ammonia by the addition of urease. Ammonia formed in this reaction was quantified by measuring the extinction of NADPH utilized in the ammonia conversion into giutamate. Plasma $\mathrm{NO}_{2}{ }^{-}$and $\mathrm{NO}_{3}{ }^{-}$concentrations were determined by a fully automated HPLC (Pharmacia, Woerden, the Netherlands) and sum (NOX) was taken. Hereto, $100 \mu \mathrm{L}$ acetonitrile was added to $50 \mu \mathrm{L}$ plasma, vortexed and centrifuged. From the clear supernatant, $60 \mu \mathrm{L}$ was mixed with $140 \mu \mathrm{L}$ Super-Q water and subsequently, $100 \mu \mathrm{L}$ injected and UV-detected on the HPLC system. The $\mathrm{NO}_{2}$ and $\mathrm{NO}_{3}$ contents are then determined by ion chromatography and UV-detected at $280 \mathrm{~nm}$. Plasma amino acids were determined by HPLC after pre-column derivatization with ophthaldialdehyde (39). Enrichments of amino acids were determined by a fully automated liquid chromatography-mass spectrometry (Thermoquest LCQ, Veenendaal, the Netherlands) system and calculated as racer-to-tracee ratios (TTR) as has previously been described (38). 


\section{Calculations}

Using the $N_{2}$-arginine tracer, the whole-body rate of appearance ( $Q R a_{A R G}$, $\mu \mathrm{mol} \bullet \mathrm{kg}^{-1} \bullet \mathrm{min}^{-1}$ ) of arginine is derived from formula $Q \mathrm{Ra}_{\mathrm{ARG}}=1 / T T R_{\mathrm{A}}$. The rate of the tracer infused is represented by $I\left(\mu \mathrm{mol} \bullet \mathrm{kg}^{-1} \bullet \mathrm{min}^{-1}\right)$ and $T T R_{A}$ represents the tracer to tracee ratio of the isotopic tracer in the arterial plasma (41). The "gut" is defined as the total of all portal-drained organs which, besides for the spleen, stomach and pancreas, largely represent the intestines. The splanchnic area is the sum of the portal-drained viscera and liver, therefore, calculations on liver are made by subtracting portal-drained viscera from splanchnic values. Substrate metabolism across the hindquarter, the gut and the liver was calculated in a two-compartment model as was described previously (41). Calculations on muscle kinetics were made by assuming that the hindquarter

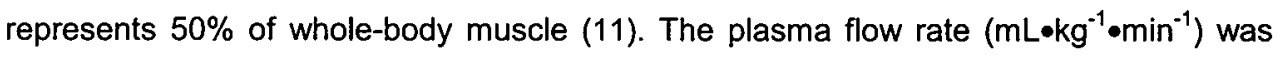
calculated using the PAH indicator-dilution technique (37). Substrate net balance (NB; $\mathrm{nmol} \bullet \mathrm{kg}^{-1} \bullet \mathrm{min}^{-1}$ ) was calculated by multiplying the mean plasma flow with the difference between the venous and arterial plasma concentration of the amino acid (NB $=$ flow $\times$ (V] - $[A])$ ). A positive NB represents net efflux from the organ and a negative NB net influx into the organ. The disposal rate $\left(\mathrm{nmol} \bullet \mathrm{kg}^{-1} \bullet \mathrm{min}^{-1}\right)$ of an amino acid represents its total rate of disappearance by conversion into other compounds or incorporation into protein. Disposal can be calculated by using the tracer disappearance across that organ. For that purpose, the net balance of the tracer $(\mathrm{nb})$ across an organ is calculated from arterial-venous tracer concentration differences in the plasma. Tracer concentrations are the product of tracer to tracee ratio (TTR) and tracee (or amino acid) concentration in the plasma, respectively; $n b=$ flow $\times\left([A] \times T T R_{A}-[V] \times T T R_{V}\right)$. Amino acid disposal is subsequently calculated by dividing the net balance of the tracer with the venous enrichment or TTR (Disposal $=n b / T T R_{V}$ ). The venous enrichment was used for the calculation of the disposal rate because it best approaches the intracellular enrichment (precursor pool) of the organ (2). Since the net balance of a substrate across an organ is the difference between disposal and production by that organ, the production is calculated by subtracting the net balance from the disposal rate. The rate of organ NO synthesis can be calculated from conversion of $N_{2}$-arginine to $N_{1}$-citrulline (6) across that organ, therefore, is calculated by using the venous-arterial organ net balance of $\mathrm{N}_{1}$ citrulline tracer divided by the arterial enrichment of $\mathrm{N}_{2}$-arginine. Because of possible disposal of $N_{1}$-citrulline across the organ, tracer $n b$ of $N_{1}$-citrulline and, therefore, calculated NO production is an underestimate of true NO production. 


\section{Statistics}

Results are presented as means \pm SEM. Because very similar results were obtained at Day 0 between the CON and the ET group, pigs were pooled into one CON 0 group. The data were subjected to an analysis of variance (ANOVA) to compare group differences after 24-h endotoxin (ET 24 vs. CON 24) and to test changes in time within the groups. Significance from zero was tested by the Wilcoxon's non-parametric test. Levels of significance were set at $P<0.05$.

\section{Results}

The body temperature of the pigs (Figure 2a) rose to a maximum at $5 \mathrm{~h}$ after initiation of endotoxin and gradually returned to control values at $24 \mathrm{~h}$. The heart rate of the pigs (Figure $2 \mathrm{~b}$ ) increased to a maximum after about $4 \mathrm{~h}$ of endotoxin infusion and almost stabilized to control values at 8-h endotoxin infusion. After an initial decrease in MAP after $1.5 \mathrm{~h}$ of endotoxin infusion, MAP steadily returned to baseline values at $4.5-5 \mathrm{~h}$ (Figure 2c). After 5-h endotoxin infusion, MAP again fall below baseline values and remained low at $24 \mathrm{~h}$. Changes in cardiac output $(\mathrm{CO})$ were followed in time in a separate pilot study using 6 endotoxin-treated pigs. $\mathrm{CO}$ of the pigs progressively increased after initiation of endotoxin and remained significantly elevated at $24 \mathrm{~h}$ (Figure 2d). These findings show that the experimental protocol induced the symptoms of hyperdynamic endotoxemia.

Table 1. Temperature, hematocrit, arterial blood gas values and plasma flow in endotoxemic pigs and controls.

\begin{tabular}{|c|c|c|c|}
\hline & CON 0 & CON 24 & ET 24 \\
\hline Temperature $\left({ }^{\circ} \mathrm{C}\right)$ & $37.9 \pm 0.2$ & $37.6 \pm 0.3$ & $39.9 \pm 0.2^{* *}$ \\
\hline Hematocrit (\%) & $28.9 \pm 0.6$ & $27.9 \pm 1$ & $24.5 \pm 0.8^{* *}$ \\
\hline $\mathrm{pH}$ & $7.4 \pm 0.1$ & $7.3 \pm 0.1$ & $7.4 \pm 0.1$ \\
\hline $\mathrm{PaCO}_{2}(\mathrm{kPa})$ & $5.7 \pm 0.2$ & $5.2 \pm 0.6$ & $4.7 \pm 0.1^{*}$ \\
\hline $\mathrm{PaO}_{2}$ & $12.6 \pm 0.5$ & $11.9 \pm 0.8$ & $13.0 \pm 0.8$ \\
\hline $\mathrm{HCO}_{3}^{-}(\mathrm{mM})$ & $27.5 \pm 1.0$ & $21.8 \pm 3.2$ & $24.1 \pm 1.1$ \\
\hline $\mathrm{O}_{2}$ saturation $(\%)$ & $97.2 \pm 0.3$ & $96.7 \pm 0.9$ & $97.2 \pm 0.4$ \\
\hline \multicolumn{4}{|c|}{ Flow $\left(m L \cdot k^{-1} \cdot \min ^{-1}\right)$} \\
\hline PDV & $33.5 \pm 3.5$ & $26.3 \pm 4.2$ & $34.0 \pm 1.5$ \\
\hline Liver & $45.7 \pm 4.4$ & $48.1 \pm 4.4$ & $55.9 \pm 4.3$ \\
\hline Muscle & $28.1 \pm 3.6$ & $31.7 \pm 7.2$ & $42.6 \pm 4.4^{* *}$ \\
\hline
\end{tabular}

Data are mean \pm s.e.mean. During fasting before (CON 0 ) and after 24-h saline (CON 24) or endotoxin (ET 24) infusion. $\mathrm{PaCO}_{2}$ and $\mathrm{PaO}_{2}$ arterial $\mathrm{CO}_{2}$ and $\mathrm{O}_{2}$ partial pressure, $\mathrm{HCO}_{3}{ }^{-}$; arterial bicarbonate. Statistics: no significance differences between the CON 24 and CON 0 group were observed, ${ }^{*} P<0.05$, ${ }^{\star *} P<0.01$; ET 24 vs. CON. 
a

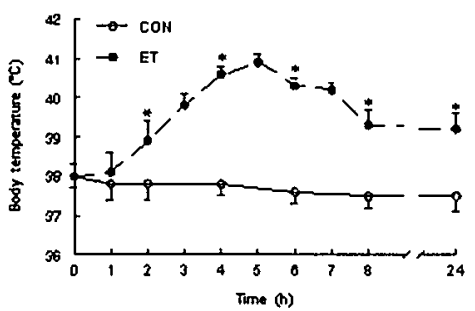

b

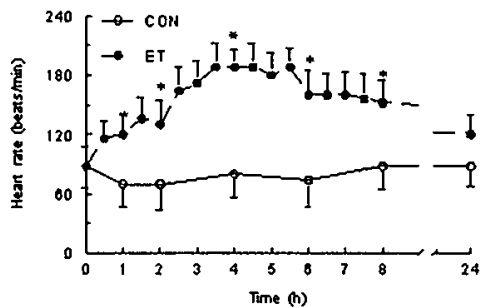

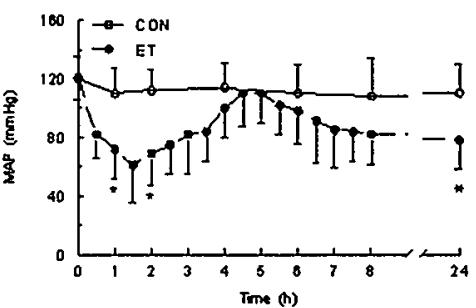

d

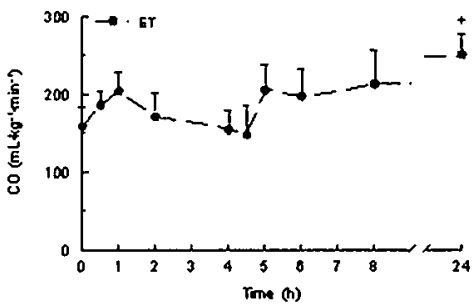

Figure 2. Data are mean \pm s.e.mean. (a) Body temperature (CON, $n=7 ; E T, n=7$ ), (b) heart rate (CON, $n=4$; $E T, n=5)$ and (c) mean arterial blood pressure or MAP (CON, $n=3 ; E T, n=3$ ) are followed during 24-h saline or endotoxin infusion. (d) Cardiac output (CO) was measured in a different endotoxin-treated group (ET, $n=6)$. Statistics: ${ }^{*} P<0.05$; ET vs. CON group and ${ }^{*} P<0.05$; vs. baseline values $(0 \mathrm{~h})$.

\section{Organ plasma flow and arterial blood gas values}

Table 1 shows that the arterial blood gas values and plasma flow across the gut, liver and hindquarter were not affected within the control group by the 24-h starvation plus fluid resuscitation period. The arterial $\mathrm{PaCO}_{2}$ was decreased by $24-\mathrm{h}$ endotoxin challenge when compared to saline infused pigs whereas other blood gas values did not significantly change (Table 1). The plasma flow across the hindquarter increased during endotoxin infusion while gut and liver flow tended to increase (both $P=0.09$ ).

\section{Whole-body parameters}

The 24-h fasting plus saline infusion period within the control group decreased the arterial concentration of $\mathrm{NO}_{2}{ }^{-} / \mathrm{NO}_{3}{ }^{-}$, ornithine, citrulline and urea but increased that of arginine (Table 2). Endotoxin infusion did not affect arterial concentration of $\mathrm{NO}_{2}{ }^{-} / \mathrm{NO}_{3}{ }^{-}$ and ornithine, increased the urea concentration and reduced the arginine concentration. A drop in whole-body rate of arginine appearance was observed after pigs were fasted and resuscitated for $24 \mathrm{~h}$ (Figure 3 ) while endotoxin induced a rise in whole-body arginine appearance compared to controls. 


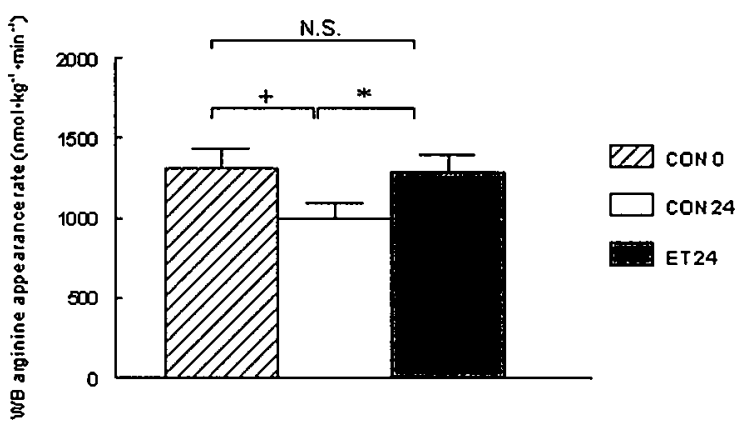

Figure 3. Whole-body appearance rate of arginine in the postabsorptive state before (CON 0 ) and $24 \mathrm{~h}$ after starvation plus saline (CON 24) or endotoxin (ET 24) infusion. Statistics: ${ }^{+} P<0.05 ;$ CON 24 vs. CON 0 and " $P$ $<0.05$; ET 24 vs. CON 24. N.S.: not significant.

\section{Gut}

No significant net balances of urea (not shown) and ornithine (Figure 4) across the gut were detected $(P>0.05)$. Intervention with endotoxin exerted no effect on the intestinal urea, ornithine or citrulline efflux. Endotoxin infusion reduced the arginine efflux 6-fold. Figure 5 shows that the arginine efflux from the gut in the endotoxin-treated animals decreased due to increased arginine disposal (or disappearance) and unchanged arginine production (or appearance) compared to corresponding control animals. NO production by the gut, measured from $\mathrm{N}_{2}$-arginine to $\mathrm{N}_{1}$-citrulline conversion, was not significantly different from zero $(P>0.05)$ in both the control groups (Figure 6$)$. In the endotoxin-treated pigs, the increase in arginine disposal $\left(174 \mathrm{nmol} \bullet \mathrm{kg}^{-1} \bullet \mathrm{min}^{-1}\right)$ matched the increase in NO production (171 $\mathrm{nmol}^{\circ} \mathrm{kg}^{-1} \bullet \mathrm{min}^{-1}$; Figure 6$)$.

Table 2. Arterial concentrations in endotoxemic pigs and controls.

\begin{tabular}{lccc}
\hline & $\mathrm{CON} 0$ & $\mathrm{CON} 24$ & ET 24 \\
\hline $\mathrm{NO}_{2}{ }^{-} \mathrm{NO}_{3}{ }^{-}$ & $153 \pm 38$ & $54 \pm 15^{+*}$ & $33 \pm 6$ \\
Urea & $3535 \pm 451$ & $1368 \pm 457^{+*}$ & $2729 \pm 227^{\star *}$ \\
Ornithine & $107 \pm 9$ & $79 \pm 10^{+}$ & $58 \pm 4$ \\
Citrulline & $79 \pm 4$ & $58 \pm 3^{+*}$ & $42 \pm 4^{\star \star}$ \\
Arginine & $92 \pm 4$ & $106 \pm 4^{+}$ & $70 \pm 3^{* \star}$ \\
$\mathrm{HCO}_{3}{ }^{\circ}(\mathrm{mM})$ & $27.5 \pm 1.0$ & $21.8 \pm 3.2$ & $24.1 \pm 1.1$ \\
\hline
\end{tabular}

Data are mean $\pm \mathrm{SEM}$ in $\mu \mathrm{M}$ except for $\mathrm{HCO}_{3}{ }^{-}$in $\mathrm{mM}$. During fasting before (CON 0 ) and after $24-\mathrm{h}$ saline (CON 24) or endotoxin (ET 24) infusion. Statistics: ${ }^{+} P<0.05,{ }^{+4} P<0.01 ;$ CON 24 versus CON $0,{ }^{*} P<0.05$, ${ }^{* \star} P<0.01$; ET 24 versus CON 24. 
Gut

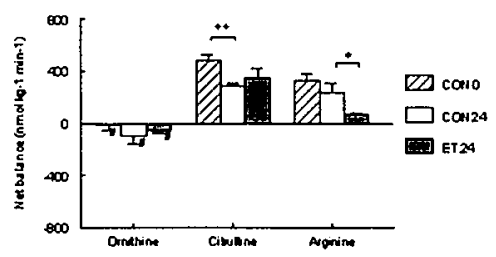

Liver

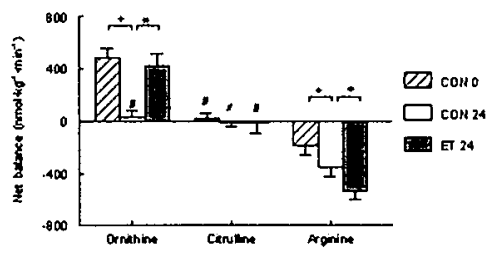

Hindquarter

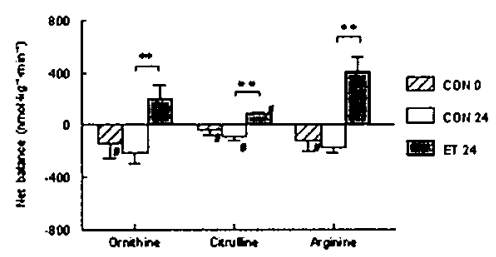

Figure 4. Net balance ( $+=$ efflux, - = influx) of, ornithine, citrulline and arginine across the gut, liver and hindquarter in the postabsorptive state before (CON 0 ) and $24 \mathrm{~h}$ after starvation plus saline (CON 24) or endotoxin (ET 24) infusion. Statistics: ${ }^{+} P<0.05,{ }^{++} P<0.01$; CON 24 vs. CON 0 . ${ }^{\star} P<0.05,{ }^{\star \star} P<0.01$; ET 24 vs. CON 24 and ${ }^{\#} P$ $>0.05$; not different from zero.
Gut

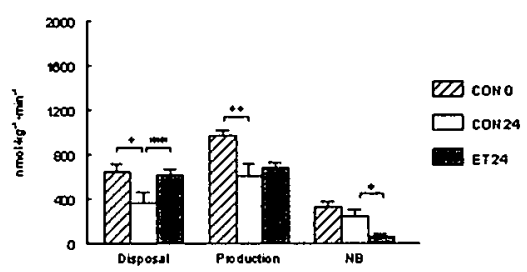

LNer

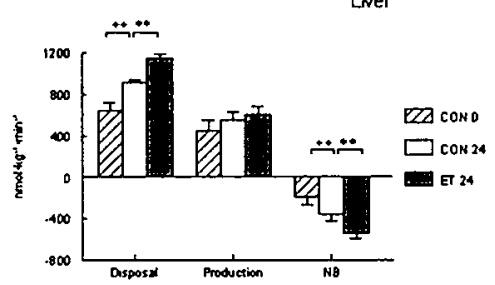

Hindquarter

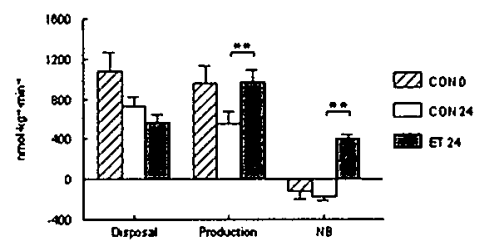

Figure 5. Disposal, production and net balance (+ $=$ efflux) of arginine across the gut, liver and hindquarter in the postabsorptive state before (CON 0 ) and $24 \mathrm{~h}$ after starvation plus saline (CON 24) or endotoxin (ET 24) infusion. Statistics: ${ }^{+} P<0.05,{ }^{++} P<0.01$; CON 24 vs. CON $0,{ }^{*} P<0.05,{ }^{* *} P<0.01$; ET 24 vs. CON 24 and ${ }^{\sharp} P$ $>0.05$; not different from zero.

\section{Liver}

The endotoxin treatment tended to increase the liver urea efflux from $2.9 \pm 0.7$ in control pigs to $7.4 \pm 1.7 \mu \mathrm{mol} \bullet \mathrm{kg}^{-1} \bullet \mathrm{min}^{-1}$ although this was not significant $(P=0.08)$. Ornithine efflux also increased by endotoxin infusion (Figure 4). No significant citrulline net balance across the liver was detectable. The influx of arginine into the liver significantly increased upon 24-h starvation and even more when endotoxin was infused. These effects could be ascribed to an increased disposal of arginine, whereas arginine production remained unaffected (Figure 5). In the endotoxin-treated animals liver NO synthesis was significantly enhanced (Figure 6). Increased arginine disposal closely matched increased NO synthesis. However, because no correction was made for $N_{1}$-citrulline tracer uptake 
across the liver, estimated NO production probably is an underestimate of true NO production.

\section{Hindquarter}

The net balance of urea across the hindquarter was not significant $(P>0.05)$ in control and endotoxin groups (not shown). When pigs were treated with endotoxin, hindquarter ornithine, citrulline, and arginine influx turned into efflux (Figure 4). Whereas fasting and fluid resuscitation tended to reduce arginine production from the hindquarter $(P=0.07)$, endotoxin intervention significantly increased arginine production relative to the corresponding controls. Production of arginine by the hindquarter accounted for approximately $60 \%$ of the systemic whole-body arginine production, assuming that the hindquarter represents $50 \%$ of the whole-body muscle. The net release of arginine from the hindquarter muscle $\left(\sim 400 \mathrm{nmol} \bullet \mathrm{kg}^{-1} \bullet \mathrm{min}^{-1}\right)$ covered all of the arginine net taken up by the splanchnic region. The measured production of NO by the hindquarter was not significantly different from zero in any of the groups (Figure 6).

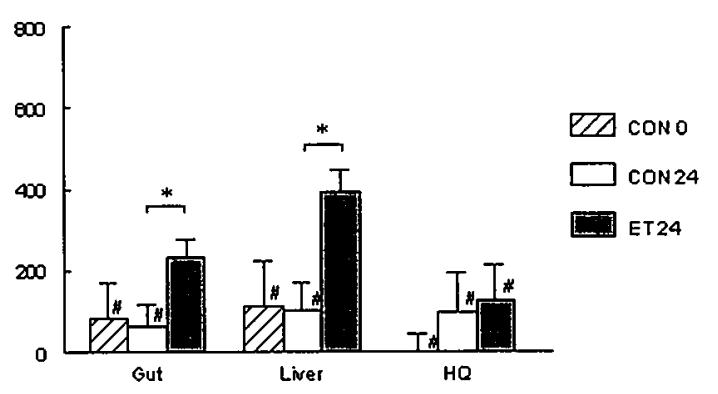

Figure 6 . Nitric oxide production by the gut, liver and hindquarter in the postabsorptive state before (CON 0 ) and $24 \mathrm{~h}$ after starvation plus saline (CON 24) or endotoxin (ET 24) infusion. Statistics: ${ }^{*} P<0.05 ;$; $E T 24$ vs. CON 24 and $" P>0.05$; not different from zero.

\section{Discussion}

In order to understand organ NO production and requirements for arginine as substrate for NO during sepsis, we performed a study in pigs in which we used stable isotope techniques to quantify the metabolic interrelationships between arginine and NO in gut, liver and hindquarter. The observed reduced MAP, elevated heart rate, cardiac output and slightly increased blood flow to the organs represent a hemodynamic response characteristic of a hyperdynamic state of compensated sepsis. Our observation that the increases in gut and liver arginine disposal correspond with increases in NO production during endotoxemia support the hypothesis that arginine metabolism is strongly involved in the regulation of $\mathrm{NO}$ production. 
The $\mathrm{NO}_{x}$ levels did not rise in the endotoxemic pigs despite augmented organ NO production measured by isotope conversion. Elevated plasma levels of $\mathrm{NO} x$ were postulated to reflect enhanced NO production in sepsis (16). However, such increases could well reflect loss of circulating plasma volume due to reduced peripheral vascular resistance and impaired renal clearance, prominent features of insufficiently fluidsupported sepsis (4). In this experimental model, endotoxemic pigs were resuscitated with saline. Preserved plasma volume together with maintained renal clearance for that reason may, in this model, have concealed endotoxin-induced increases in plasma $\mathrm{NO}_{\mathrm{x}}$ levels. Since $\mathrm{NO}_{\mathrm{x}}$ concentrations fluctuate with the patho-physiologic condition they do not appear to be a reliable reflection of the NO production rate (23).

In the gut, apart from citrulline release, the zonal distribution of urea cycle enzymes favors the production of arginine from citrulline accounting for the observed net arginine efflux by the gut, which is known to be unique for the younger pig (42). The iNOS enzyme is activated by synergistic action of cytokines and endotoxin (17). In the intestinal tract, iNOS was predominantly found in the esophagus, duodenum, jejunum, ileum, and colon, but not in the stomach its expression reaching a maximum at $4 \mathrm{~h}$ after endotoxin challenge (7). In the liver, iNOS expression is identified in hepatocytes, Kupffer cells and stellate cells, its mRNA expression peaking $6 \mathrm{~h}$ after stimulation $(5,27)$. Activity of iNOS may be present for several hours, still being present at $24 \mathrm{~h}$ after endotoxin challenge $(7$, $21,29,33)$. It is therefore most likely that the rise in visceral and hepatic NO synthesis that we observed in this model of endotoxemia results from endotoxin-induced iNOS expression.

Increased de novo synthesis of arginine from citrulline in gut and liver cells is an essential tool to meet the substrate demand for the increased NO production by iNOS (20). Such an arrangement however would result in decreased gut and liver arginine production, which we did not observe. The net arginine consumption in the gut and liver was predominantly regulated by enhanced disposal or consumption and not by decreased production of arginine. Endotoxins and cytokines are known to increase arginine transport activity via the CAT2B system, expression of which is found coinduced with iNOS in different types of cell (43). Under endotoxemic conditions the gut and liver may, for that reason, acquire arginine by increasing its capacity of active arginine transport. Such an arrangement may support important arginine-utilizing pathways including NO synthesis during sepsis. Such increased hepatic arginine transport activity accompanying increased NO production was found to occur in endotoxin-treated rats $(22,36)$.

Relatively high levels of the high $\mathrm{Km}$ enzyme arginase $\|$ are expressed the gut and arginase I in the liver. In macrophages, endotoxin was found to simultaneously activate 
arginase and iNOS enzyme expression $(34,40)$. Supposing this to be the case, increased arginine disposal may not only represent increased iNOS-mediated, but also arginase-mediated conversion. However, arginase and, therefore, arginine catabolism has been postulated to be differentiated between compartments in pig enterocytes (8). Also within the liver, a functional specialization with different zonation patterns of the urea cycle enzymes may be present $(12,30)$. It therefore seems likely that arginase and NOS enzyme activity are regulated independently, thus, increased arginine disposal for NO production not necessarily reflect increased arginase activity. Since enhanced NO synthesis in the gut and liver during endotoxemia stoichiometrically matched increased arginine disposal, the latter appears to represent, at least in part, arginine utilization for NO production.

In the skeletal muscle, expression of inducible iNOS is probably of marginal significance (15). Accordingly, we found no significant elevation of NO synthesis in the hindquarter after 24-h of endotoxin infusion. Increased arginine production from the hindquarter most likely represents accelerated protein degradation, which is present within the skeletal muscle under endotoxemic conditions (3). We showed that muscle arginine production largely determines whole-body production during fasting endotoxemia. Previously we showed that under prolonged fasting conditions, arginine production derived from muscle protein degradation is a larger source of arginine than renal de novo synthesis, the muscle contributing twice as much as the kidneys to the whole-body arginine appearance (in press, Bruins et al, Am J Clin Nutr, 2001). Therefore, by means of protein degradation, the muscle is a main supplier of arginine precursor for accelerated splanchnic NO synthesis. The rate of arginine released by the hindquarter accounted for all of the arginine taken up by the splanchnic region. It has indeed been proposed by Beaumier et al. (1) that the short-term maintenance of arginine homeostasis is largely dependent on the catabolic side of arginine metabolism, rather than on the anabolic side.

Extracellular arginine can become rate limiting as a substrate for the enhanced production of NO by the iNOS isoform $(14,32,35)$. Plasma levels of arginine were lower in the endotoxemic than in the control pigs despite elevated whole-body production. During 24-h endotoxin infusion, extracellular arginine concentration dropped from 120 to $70 \mu \mathrm{M}$, reaching the half-saturating concentration of $60-70 \mu \mathrm{M}$ for iNOS-dependent NO synthesis (18). The NO synthesis by the gut and liver at 24-h endotoxin infusion, however, appears not to be limited by the low circulating arginine levels. Increased arginine disposal rather than reduced arginine production from de novo synthesis mediated the net arginine uptake in the gut and liver. When arginine substrate availability becomes limiting, increased cellular transport of arginine may meet the increased organ arginine requirement for enhanced NO synthesis during endotoxemia. The current findings indicate that enhanced arginine disposal by the gut and liver during endotoxemia 
is associated with increased NO production in a quantitative manner, whereas muscle functions as a main supplier of arginine. In vivo organ NO measurements may contribute to a better delineation of the involvement of $N O$ in endotoxemia and help in the development of new therapeutic strategies aiming at NO targeting in the treatment of sepsis.

\section{References}

1. Beaumier, L., L. Castillo, Y. M. Yu, A. M. Ajami, and V. R. Young. Arginine: new and exciting developments for an "old" amino acid. Biomed Environmental Sci 9: 296-315, 1996.

2. Biolo, G., R. Y. D. Fleming, S. Maggi, and R. R. Wolfe. Transmembrane transport and intracellular kinetics of amino acids in human skeletal muscle. Am J Physiol 268: E75-E84, 1995.

3. Biolo, G., G. Toigo, B. Ciocchi, R. Situlin, F. Iscra, A. Gullo, and G. Guarnieri. Metabolic response to injury and sepsis: changes in protein metabolism. Nutrition 13: 52S-57S, 1997.

4. Bock, H. A. Pathophysiology of acute renal failure in septic shock: from prerenal to renal failure. Kidney Int Supp/ 64: S15-8., 1998.

5. Cantoni, L., F. Di Nicolantonio, D. Barelli, M. Rizzardini, and F. De Matteis. Modulation of cerebellar and hepatic nitric oxide synthase by exogenous arginine and endotoxin. Nitric Oxide 5: 198-207., 2001.

6. Castillo, L., M. Sanchez, J. Vogt, T. E. Chapman, T. C. DeRojas-Walker, S. R. Tannenbaum, A. M. Ajami, and V. R. Young. Plasma arginine, citrulline, and ornithine kinetics in adults, with observations on nitric oxide synthesis. Am J Physiol 268: E360-E367, 1995.

7. Chen, K., M. Inoue, and A. Okada. Expression of inducible nitric oxide synthase mRNA in rat digestive tissues after endotoxin and its role in intestinal mucosal injury. Biochem Biophys Res Commun 224: 703-8., 1996.

8. Davis, P. K., and G. Wu. Compartmentation and kinetics of urea cycle enzymes in porcine enterocytes. Comp Biochem Physiol B 119: 527-537, 1998.

9. Desmukh, D. R., V. S. Ghole, B. Marescau, and P. P. De Deyn. Effect of endotoxemia on plasma and tissue levels of nitric oxide metabolites and guanidino compounds. Arch Physiol Biochem 105: 32-37, 1997.
10. Deutz, N. E. P., P. L. M. Reijven, G. Athanasas, and P. B. Soeters. Post-operative changes in hepatic, intestinal, splenic and muscle fluxes of amino acids and ammonia in pigs. Clin Sci 83 : 607-614, 1992.

11. Deutz, N. E. P., A. J. M. Wagenmakers, and P. B. Soeters. Discrepancy between muscle and whole body protein turnover. Cur Opin Clin Nutr Metab Care 2: 29-32, 1999.

12. Dingemanse, M. A., W. J. de Jonge, P. A. J. de Boer, M. Mori, W. H. Lamers, and A. F. M. Moorman. Development of the ornithine cycle in rat liver: zonation of a metabolic pathway. Hepatology 24: 407-411, 1996.

13. Dodds, W. J. The pig model for biomedical research. Federation Proc 41: 247-256, 1982.

14. Durante, W., L. Liao, I. Iftikhar, W. E. O'Brien, and A. I. Schafer. Differential regulation of Larginine transport and nitric oxide production by vascular smooth muscle and endothelium. Circ Res 78: 1075-82., 1996.

15. el-Dwairi, Q., A. Comtois, Y. Guo, and S. N. Hussain. Endotoxin-induced skeletal muscle contractile dysfunction: contribution of nitric oxide synthases. Am J Physiol 274: C770-9, 1998.

16. Evans, T., A. Carpenter, H. Kinderman, and J. Cohen. Evidence of increased nitric oxide production in patients with the sepsis syndrome. Circ Shock 41: 77-81, 1993.

17. Geller, D. A., A. K. Nussler, M. Di Silvo, C. J. Lowenstein, R. A. Shapiro, S. C. Wang, R. L. Simmons, and T. R. Billiar. Cytokines, endotoxin, and glucocorticoids regulate the expression of inducible nitric oxide synthase in hepatocytes. Proc Natl Acad Sci USA 90: 522526, 1993.

18. Granger, D. L., J. B. Hibbs, Jr., J. R. Perfect, and D. T. Durack. Metabolic fate of L-arginine in relation to microbiostatic capability of murine macrophages. J Clin Invest 85: 264-73, 1990. 
19. Grisham, M. B., D. Jourd'Heuil, and D. A. Wink. Nitric oxide. I. Physiological chemistry of nitric oxide and its metabolites:implications in inflammation. Am J Physiol 276: G315-21, 1999.

20. Hattori, Y., S. Shimoda, and S. S. Gross. Effect of lipopolysaccharide treatment in vivo on tissue expression of argininosuccinate synthetase and argininosuccinate lyase mRNAs: relationship to nitric oxide synthase. Biochem Biophys Res Commun 215: 148-53, 1995.

21. Heba, G., T. Krzeminski, M. Porc, J. Grzyb, A. Ratajska, and A. Dembinska-Kiec. The time course of tumor necrosis factor-alpha, inducible nitric oxide synthase and vascular endothelial growth factor expression in an experimental model of chronic myocardial infarction in rats. $J$ Vasc Res 38: 288-300., 2001.

22. Hwang, T. L., J. T. Yang, and Y. T. Lau. Arginine-nitric oxide pathway in plasma membrane of rat hepatocytes during early and late sepsis. Crit Care Med 27: 137-141, 1999.

23. Kirkeboen, K. A., and O. A. Strand. The role of nitric oxide in sepsis-an overview. Acta Anaesthesiol Scand 43: 275-88, 1999.

24. Laszlo, F., B. J. R. Whittle, and S. Moncada. Time-dependent enhancement or inhibition of endotoxin-induced vascular injury in rat intestine by nitric oxide synthase inhibitors. $\mathrm{Br} J$ Pharmacol 111: 1309-1315, 1994.

25. Lortie, M. J., S. Ishizuka, D. Schwartz, and R. C. Blantz. Bioactive products of arginine in sepsis: tissue and plasma composition after LPS and iNOS blockade. Am J Physiol Cell Physiol 278: C1191-9., 2000.

26. Miller, E. R., and D. E. Ullrey. The pig as a model for human nutrition. Annu Rev Nutr 7 361-82, 1987.

27. Muriel, $P$. Regulation of nitric oxide synthesis in the liver. J Appl Toxicol 20: 189-95., 2000.

28. Muscara, M. N., and J. L. Wallace. Nitric Oxide. $V$. therapeutic potential of nitric oxide donors and inhibitors. Am J Physiol 276: G1313-6, 1999.

29. Niwa, M., S. Inao, M. Takayasu, T. Kawai, Y. Kajita, T. Nihashi, R. Kabeya, T. Sugimoto, and J. Yoshida. Time course of expression of three nitric oxide synthase isoforms after transient middle cerebral artery occlusion in rats. Neurol Med Chir (Tokyo) 41: 63-72; discussion 72-3., 2001.

30. O'Sullivan, D., J. T. Brosnan, and M. E. Brosnan. Hepatic zonation of the catabolism of arginine and ornithine in the perfused rat liver. Biochem J 330: 627-632, 1998.
31. Palm, U., W. Boemke, D. Bayerl, N. Schnoy, N. C. Juhr, and $H$. W. Reinhardt. Prevention of catheter-related infections by a new, catheterrestricted antibiotic filling technique. Lab Anim 25: 142-52, 1991.

32. Pastor, C. M., S. M. Morris, Jr., and T. R. Billiar. Sources of arginine for induced nitric oxide synthesis in the isolated perfused liver. $A m \mathrm{~J}$ Physiol 269: G861-G866, 1995.

33. Preiser, J. C., H. Zhang, B. Vray, A. Hrabak, and $J$. L. Vincent. Time course of inducible nitric oxide synthase activity following endotoxin administration in dogs. Nitric Oxide 5: 208-11. 2001.

34. Salimuddin, A. Nagasaki, T. Gotoh, H. Isobe, and M. Mori. Regulation of the genes for arginase isoforms and related enzymes in mouse macrophages by lipopolysaccharide. Am JPhysiol 277: E110-7, 1999.

35. Schott, C. A., G. A. Gray, and J. C. Stoclet. Dependence of endotoxin-induced vascular hyporeactivity on extracellular L-arginine. $\mathrm{Br} J$ Pharmacol 108: 38-43., 1993

36. Stark, M. E., A. J. Bauer, and J. H. Szurszewski. Effect of nitric oxide on circular muscle of the canine small intestine. J Physiol (Lond) 444: 743-61, 1991.

37. Ten Have, G. A. M., M. C. F. Bost, J. C. A. W. Suyk-Wierts, A. E. J. M. van den Bogaart, and N. E. P. Deutz. Simultaneous measurement of metabolic flux in portally-drained viscera, liver, spleen, kidney and hindquarter in the conscious pig. Lab Anim 30: 347-358, 1996.

38. Van Eijk, H. M. H., D. R. Rooyakkers, and N. E. $P$. Deutz. Determination of amino acid isotope enrichment using liquid chromatography-mass spectrometry. Anal Biochem 271: 8-17, 1999.

39. Van Eijk, H. M. H., D. R. Rooyakkers, and N. E. $P$. Deutz. Rapid routine determination of amino acids in plasma by high-performance liquid chromatography with a 2-3 $\mu \mathrm{M}$ Spherisorb ODS II column. J Chromatogr 620: 143-148, 1993.

40. Wang, W. W., C. P. Jenkinson, J. M. Griscavage, R. M. Kern, N. S. Arabolos, R. E. Byrns, S. D. Cederbaum, and L. J. Ignarro. Coinduction of arginase and nitric oxide synthase in murine macrophages activated by lipopolysaccharide. Biochem Biophys Res Comm 210: 1009-1016, 1995.

41. Wolfe, R. R. Radioactive and stable isotope tracers in biomedicine. Principles and practice of kinetic analysis. New York: Wiley-Liss, 1992.

42. Wu, G., and D. A. Knabe. Arginine synthesis in enterocytes of neonatal pigs. Am J Physiol 269: R621-R629, 1995.

43. Wu, G., and S. M. Morris, Jr. Arginine metabolism: nitric oxide and beyond. Biochem $J$ 336: 1-17, 1998. 
L-ARGININE SUPPLEMENTATION IN HYPERDYNAMIC ENDOTOXEMIC PIGS: EFFECT ON NO SYNTHESIS BY THE DIFFERENT ORGANS

\author{
Maaike J Bruins \\ Peter $B$ Soeters \\ Wouter H Lamers * \\ Alfred J Meijer * \\ Nicolaas EP Deutz
}

Department of Surgery, Maastricht University, The Netherlands

-Department of Anatomy \& Embryology, University of Amsterdam, AMC, The Netherlands 


\section{Abstract}

Under septic conditions, the protective role of nitric oxide (NO) in the organs may become compromised at a time of increased demand as a result of decreased availability for L-arginine. It remains unknown whether supplementation with L-arginine, as a substrate, can modulate organ NO production. Pigs were challenged with Escherichia coli endotoxin (i.v.) and received fluid resuscitation (i.v.) for $24 \mathrm{~h}$ to reproduce a model of long-lasting hyperdynamic endotoxemia. Pigs were either infused L-arginine or L-alanine via the intravenous route during endotoxin and via the intragastric route after cessation of endotoxin infusion. The effects of L-arginine supplementation on NO synthesis and the relationships with arginine metabolism were determined with a stable isotope infusion protocol. Also, organ nitrite plus nitrate $\left(\mathrm{NO}_{\mathrm{x}}\right)$ fluxes were measured. Implantation of multiple catheters enabled in vivo measurements across the hindquarter muscle, the portal-drained viscera, the liver and the kidneys. The isotope conversion method showed that L-arginine intervention significantly increased NO production by the portal-drained viscera, liver and kidneys, resulting in elevated whole-body NO synthesis under endotoxemic and postendotoxemic conditions. Organ $\mathrm{NO}_{x}$ fluxes only tended to increase due to high variance among data. In this endotoxemia model, supplemental use of Larginine favored the NO synthesis in various organs.

\section{Introduction}

L-arginine is the precursor of the short-living molecule nitric oxide (NO). NO is involved in the regulation of many processes such as neurotransmission, vasodilatation and immune defense. NO plays a dual role since the effects of NO seem to depend on the quantity formed (for a thorough discussion, see: (19)). Under normal circumstances, small quantities of NO are synthesized by the constitutively expressed NO synthase isoforms (NOS-I and III) that produce NO when intracellular calcium levels increase. Elevated calcium-independent production of NO results from the expression of the inducible NOS isoform (NOS-II) that is induced by inflammatory mediators such as endotoxin or cytokines (for extended reviews, see: $(13,18)$ ). Uncontrolled production of NO can be detrimental and contribute to tissue injury, hypotension and myocardial failure (5). Inhibition of the excessive NO production by using non-specific NOS inhibitors during sepsis entails, however, the simultaneous inhibition of the basal NO production that mediates "normal functioning", thereby resulting in disturbed tissue perfusion (19) and increased tissue injury (20). An increasing number of studies suggest a beneficial effect 
of L-arginine supplementation under disease conditions, including anti-catabolic effects and improved cell-mediated immune response (reviewed in: (8)).

In septic animals and humans with severe metabolic stress such as sepsis, plasma Larginine levels are markedly decreased, suggesting that the endogenous synthesis and dietary supply of L-arginine do not meet the increased demand (10). Since intracellular Larginine availability is largely determined by L-arginine transporters with a $K_{M}$ in the same range as circulating L-arginine concentration, L-arginine may become rate limiting as substrate for the synthesis of NO $(11,16)$. In view of the fact that basal levels of NO are crucial for maintenance of cell and organ function, it could be envisioned that L-arginine supplementation is beneficial under these circumstances. The aim of the present experiment was to explore the effects of L-arginine supplementation on NO formation and the quantitative relations to the L-arginine metabolism under both fasting endotoxemic and fed postendotoxemic conditions. Since intravenous $L$-arginine did not affect whole-body NO synthesis in non-endotoxemic pigs (unpublished results, Poeze M, 2001), the effect of L-arginine on whole-body and organ NO synthesis was only investigated in endotoxemic pigs. Under conditions with markedly elevated NOS-II expression such as sepsis (14), sustained L-arginine utilization might lead to gradual Larginine depletion. Therefore, we chose to start L-arginine supplementation several hours after the initiation of endotoxin infusion as a strategy to avoid substrate deficiency for NO synthesis. To mimic hyperdynamic sepsis, small doses of endotoxin and adequate quantities of fluid were infused over an extended period of time (9). The pig was chosen as experimental animal model, closely resembling the human physiology $(7,17)$. This model not only enables measurements on a whole-body level but also across individual organs. The L-arginine-NO metabolism could be studied by means of stable isotope techniques. In vivo studies regarding whole-body L-arginine and NO kinetics were earlier undertaken in healthy man (3) and across organs in healthy dogs (27). In addition, wholebody L-arginine and ornithine metabolism were studied in humans given L-arginineenriched diets (1). To our knowledge, the therapeutic effects of L-arginine supplementation on organ L-arginine-citrulline-NO metabolism under conditions of endotoxemia have not yet been explored.

\section{Materials and methods}

\section{Animals}

Female crossbred pigs (Yorkshire $\times$ Dutch Landrace: $20-22 \mathrm{~kg}$ body weight (bw)) were individually housed and fed $1 \mathrm{~kg}$ of regular pig feed (Landbouwbelang, Roermond, the Netherlands; $16 \%$ crude protein) daily, supporting a growth rate of approximately 300 
gram per day. Before surgery, pigs were randomly assigned to one of two treatment groups. The study was approved by the Animal Ethics Committee of Maastricht University.

\section{Surgical procedure}

The night before surgery, food was withheld from the pigs. One hour after premedication with $10 \mathrm{mg} / \mathrm{kg}$ bw intramuscular azoperone (Stresnil, Janssen pharmaceutica, Beersse, Belgium), anesthesia was induced by a mixture of $\mathrm{N}_{2} \mathrm{O}^{\prime} \mathrm{O}_{2}(1: 2)$ and halothane $(0.8 \%)$. After intubation, the pigs were intravenously administrated $6.25 \mathrm{mg} / \mathrm{kg}$ bw lincomycin. $2 \mathrm{HCl}$ (Lincomycin, A.U.V., Cuyk, the Netherlands) as bactericidal prophylaxis and $12.5 \mathrm{mg} / \mathrm{kg}$ bw spectinomycin. $\mathrm{HCl}$ lyophil (Spectinomycin, A.U.V.) as bacteriostatic prophylaxis. To avoid coagulation and as postoperative analgesia, $50 \mathrm{mg} / \mathrm{kg} \mathrm{bw}$ flunixine (Finadyne, Schering-Ploegh, Brussel, Belgium) was given. During surgery, the anesthesia was maintained by the $\mathrm{N}_{2} \mathrm{O} / \mathrm{O}_{2}$-halothane mixture and I.V. given Lactetrol (per ml: $5,76 \mathrm{mg} \mathrm{NaCl}, 0.37 \mathrm{mg} \mathrm{KCl}, 0.37 \mathrm{mg} \mathrm{CaCl}, 0.2 \mathrm{mg} \mathrm{MgCl}_{2}, 5 \mathrm{mg}$ sodium lactate, methyl parahydroxy bezoicacid, Janssen pharmaceutica). The surgical procedure has been described in detail elsewhere (22). In brief, after a midline laparotomy was performed, two catheters were cannulated into the abdominal aorta (one set just above the bifurcation (A1) and one set above the right renal vein (A2) and two in the inferior caval vein at the corresponding positions (V1 and V2, respectively). Catheters were also inserted into the portal $(P)$, hepatic $(H)$, renal $(R)$, and splenic $(S)$ vein. The $A 1$ and the $S$ catheters were used for infusion of para-aminohippuric acid (PAH), the V2 catheter for infusion of isotopes and endotoxin and the $A 2, P, H, V 1$ and $R$ catheters for blood sampling across the portal-drained viscera, the splanchnic area, the hindquarter and the kidney, respectively. Also, a gastrostomy catheter was inserted. All catheters were tunneled through the abdominal wall and skin. The pigs also underwent construction of a Bishop-Cook stoma. Briefly, $20 \mathrm{~cm}$ distal to the ligament of Treitz (where the duodenum changes into the jejunum), the jejunum was transected and the anatomical continuity of the remaining bowel reestablished by a single layer running suture (PDS 4-0 synthetic polydioxanon; Ethicon, Norderstedt, Germany) according to an end-to-side anastomosis. During recovery of the pigs, an inflated balloon catheter (size Ch.16, 30-50 ml; RüschGold, Kernen, Germany) closed the stoma to avoid leakage from the jejunum.

\section{Postoperative care}

The pigs wore a canvas harness to protect the catheters and stoma, and to allow easy handling. To support postoperative recovery of the pigs, they were fed $100 \mathrm{~g}$ of regular pig food the first and $200 \mathrm{~g}$ the second postoperative day. From the third day onwards, pigs were fed $1 \mathrm{~kg}$ of food daily ( $72 \mathrm{~g}$ protein / day). To avoid catheter tip infections and 
to maintain patency, catheters were regularly flushed with $150 \mathrm{mmol} / \mathrm{L} \mathrm{NaCl}$ (saline) and filled with a solution containing a mixture of gentamycin (20 g/L; A.U.V., Cuyk, the Netherlands) and chymotrypsin (0.225 U/L, Merck, Darmstadt, Germany) in saline. During handling, the pigs were placed in a movable cage to get accustomed to this condition. Postoperative care of the pigs was standard (22). During the whole recovery (10-15 days) and experimental period (7 days), the animals remained healthy without signs of infection.

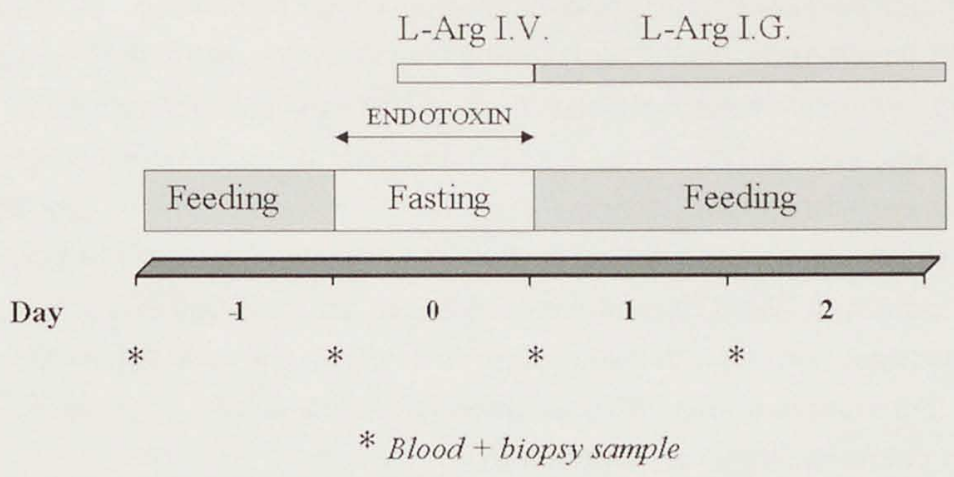

Figure 1. Schematic illustration of the experimental design. Day -1: during feeding, Day 0: after 8-h overnight fasting, Day 1: during 24-h fasting with endotoxin infusion and Day 2: during feeding and recovery from endotoxin infusion. Pigs received L-Ala (CON) or L-Arg (ARG) intervention. During Day 0 to $1 \mathrm{~L}$-Ala/LArg is intravenously (I.V.) infused whereas during Day 1 to 2 L-Ala/L-Arg is intragastrically (I.G.) infused. Blood samples and biopsies are collected at Day -1, 0, 1 and 2, one hour after stable isotope and paraaminohippuric acid infusion.

\section{Experimental protocol}

During the entire experimental protocol, blood gas values, heart rate, arterial blood pressure, weight and temperature of the pig were monitored. When the pigs were recovered from surgery, a high-protein liquid enteral nutrition (Nutrison Steriflo ProteinPlus, Nutricia, Zoetermeer, the Netherlands; $37.5 \mathrm{mg}$ protein $/ \mathrm{mL}$ ) was infused into the gastrostomy catheter via a swivel system connected to a pump. The enteral nutrition was infused at a rate of $4 \mathrm{~mL} \cdot \mathrm{kg} \mathrm{bw}^{-1} \cdot \mathrm{h}^{-1}$ ( $\sim 72 \mathrm{~g}$ protein / day) for 3 days of which the first day enteral nutrition was infused at half rate in addition to $500 \mathrm{~g}$ of their regular diet to get pigs accustomed to the enteral nutrition. On the last day of enteral feeding (Day -1) the experimental protocol is started. The experimental design is depicted in Figure 1. At Day -1 control blood samples of the postprandial state were obtained from 14 pigs. The following day (Day 0) after an 8-h overnight fast, control samples of the postabsorptive state were taken. Immediately thereafter, an infusion of LPS endotoxin from Escherichia coli (serotype 055:B5, Sigma Chemicals Co, St. Louis, MO, USA), dissolved in saline, was started at a rate of $3 \mu \mathrm{g} \bullet \mathrm{kg} \mathrm{bw}^{-1} \cdot \mathrm{h}^{-1}$ via the venous (V2) catheter for a period of $24 \mathrm{~h}$. 
All pigs received infusions with extra saline to replenish intravascular volume losses (30 mi $\cdot k g ~ b w^{-1} \cdot h^{-1}$ the first $8 \mathrm{~h}$ and $20 \mathrm{ml} \cdot \mathrm{kg} \mathrm{bw}^{-1} \cdot \mathrm{h}^{-1}$ the following $16 \mathrm{~h}$ ). Pigs were either $(n=8)$ given an intravenous infusion of L-arginine (iso-osmolar, $5.3 \mu \mathrm{mol} \bullet \mathrm{kg} \mathrm{bw}^{-1} \cdot \mathrm{min}^{-1}$, $\mathrm{pH} 7.3)$ or $(n=6)$ L-alanine as a control amino acid at an isocaloric rate $(10.6 \mu \mathrm{mol} \bullet \mathrm{kg} \mathrm{bw}$ ${ }^{1} \cdot \mathrm{min}^{-1}, \mathrm{pH} 7.3$ ) starting $8 \mathrm{~h}$ after initiation of endotoxin. The next day, after $24 \mathrm{~h}$ of endotoxin infusion, blood was sampled from the L-arginine-treated (ARG) and the Lalanine-treated (CON) group. When the intravenous infusions of endotoxin, saline and amino acid infusions were discontinued, enteral nutrition was restarted $\left(4 \mathrm{~mL} \cdot \mathrm{kg} \mathrm{bw}^{-1} \cdot \mathrm{h}^{-}\right.$ $\left.{ }^{1}\right)$. The intravenous infusion of L-arginine or L-alanine was followed up by intragastric infusion at a similar infusion rate. The following day (Day 2), $24 \mathrm{~h}$ after cessation of endotoxin, blood samples were obtained from the intragastrically L-arginine and Lalanine-treated pigs. Also at Day $-1,0,1$ and 2, mucosal biopsies were collected from the jejunum via the Bishop-Cook stoma. Body temperature and mean arterial blood pressure (MAP) were determined during 4-h and 8-h endotoxin infusion at Day 0 . The endotoxemia model has been validated in a different study in which 24-h endotoxin infusion was compared with 24-h saline infusion (unpublished results, Bruins MJ, 2001). In this study, important features of hyperdynamic endotoxemia such as increased temperature, heart rate and MAP were observed.

\section{Isotope infusion protocol}

Before the start of the infusions, background blood samples were taken. On the morning of the trial, one $h$ before start of the endotoxin infusion, primed infusions of paraaminohippuric acid (PAH, $25 \mathrm{mmol} / \mathrm{L}, \mathrm{A}$ 1422, Sigma Chemicals CO, St. Louis, MO, USA) and isotopes were performed. The PAH infusion was started at a rate of about 40 $\mathrm{mL} / \mathrm{h}$ per catheter through the $S$ and the $A 1$ catheter after an initial bolus of $5 \mathrm{~mL}$ (22). Directly after the PAH bolus infusion, a primed and constant infusion of the stable isotopes L-[guanidino- ${ }^{15} \mathrm{~N}_{2}$ ]-arginine $\left(\mathrm{N}_{2}\right.$-arginine; prime: $3.2 \mu \mathrm{mol} \bullet \mathrm{kg} \mathrm{bw}^{-1}$, infusion: 6.4 $\mu$ mol $\left.\bullet k g ~ b w^{-1} \bullet h^{-1}\right)$ and L-[ureido- $\left.{ }^{13} \mathrm{C} ; 5,5-{ }^{2} \mathrm{H}_{2}\right]$ citrulline $\left(\mathrm{C}_{1} \mathrm{D}_{2}\right.$-citrulline; prime: $1.5 \mu \mathrm{mol} \bullet \mathrm{kg}$ bw ${ }^{-1}$, infusion: $0.6 \mu$ mol $\left.\bullet \mathrm{kg} \mathrm{bw}^{-1} \cdot \mathrm{h}^{-1}\right)$ was started via the $\mathrm{V} 2$ catheter. $\mathrm{N}_{2}$-arginine and $\mathrm{C}_{1}$ $\mathrm{D}_{2}$-citrulline were purchased from Mass Trace (Woburn, MA, USA).

Steady state conditions for PAH and stable isotopes were assessed in a pilot experiment in which blood samples were collected in triplicate at 10-min intervals. Isotopic plateau was reached when isotopic enrichment values at different sites no longer increased with time during the previous $60 \mathrm{~min}$ of the infusion protocol (24). Therefore, at Day -1, 01 and 2, blood samples were collected $1 \mathrm{~h}$ after the start of PAH and isotope infusions. 


\section{Sample processing}

Directly after sampling, blood was apportioned in pre-chilled, heparinized tubes (Sarstedt, Nümbrecht, Germany). For blood gas analysis (arterial $\mathrm{pH}$, bicarbonate, $\mathrm{PO}_{2}$ and $\left.\mathrm{PCO}_{2}\right), 0.2 \mathrm{~mL}$ arterial blood was sealed airtight in heparinized $1 \mathrm{~mL}$ syringes and immediately analyzed on an automatic blood gas system (Acid Base Laboratory, Radiometer, Copenhagen, Denmark). Centrifugation was performed at $4^{\circ} \mathrm{C}$ for $5 \mathrm{~min}$ at $8,500 \mathrm{~g}$, plasma collected and kept on ice. For amino acid and isotopic enrichment analysis, $500 \mu \mathrm{L}$ of plasma was deproteinized by mixing with $20 \mathrm{mg}$ dry sulfosalicylic acid. To determine ammonia and urea, $400 \mu \mathrm{L}$ plasma was mixed with $40 \mu \mathrm{L}$ of $3.5 \mathrm{~mol} / \mathrm{L}$ trichloroacetic acid (TCA; Merck, Darmstadt, Germany) solution. For PAH determination, $300 \mu \mathrm{L}$ of whole blood was added to $600 \mu \mathrm{L}$ of $0.7 \mathrm{mmol} / \mathrm{L}$ TCA solution, thoroughly mixed, centrifuged and supernatant collected. Tissue biopsies of the jejunum were collected on ice and rinsed in saline. All plasma and tissue samples were frozen in liquid nitrogen and stored at $-80^{\circ} \mathrm{C}$ until use.

\section{Biochemical analysis}

Plasma PAH, ammonia and urea were detected spectrophotometrically on an automated analysis system (Cobas Mira-S, Hoffmann-La Roche, Basel, Switzerland) by standard enzymatic methods, using commercially available kits (6). Plasma $\mathrm{NO}_{2}{ }^{-}$and $\mathrm{NO}_{3}{ }^{-}$were determined by a fully automated High Performance Liquid Chromatography system (Pharmacia, Woerden, the Netherlands), using the USA Environmental Protection Agency B-1011 method. The sum of $\mathrm{NO}_{2}{ }^{-}$and $\mathrm{NO}_{3}{ }^{-}$concentrations $(\mathrm{NO})$ was taken. Concentrations and enrichments of amino acids were calculated as tracer-to-tracee ratios (TTR) and determined by a fully automated liquid chromatography-mass spectrometry (LC-MS, Thermoquest LCQ, Veenendaal, the Netherlands) system after pre-column derivatization with o-phthaldialdehyde (24). Tissue was deproteinized by adding approximately $30 \mathrm{mg}$ tissue to $400 \mu \mathrm{L} 5 \%$ SSA for amino acid and polyamine determinations, and to $400 \mu \mathrm{L} 10 \%$ TCA for ammonia determinations. Subsequently, tissue was homogenized by beating for $30 \mathrm{~s}$ with $300 \mathrm{mg}$ glass beads (Biospeck, Bartlesville, OK, USA) in a Mini-Beadbeater (Biospeck). The homogenate was centrifuged at $11,000 \mathrm{~g}$ at $4{ }^{\circ} \mathrm{C}$ for $10 \mathrm{~min}$ and supernatant collected. Amino acid concentrations were determined by $\operatorname{HPLC}(23,24)$.

\section{Calculations}

The whole-body rate of appearance (in $\mu \mathrm{mol} \cdot \mathrm{kg} \mathrm{bw}^{-1} \cdot \mathrm{min}^{-1}$ ) of $L$-arginine (Q Ra ${ }_{A R G}$ ) and citrulline (Q Ra $\mathrm{cIT}$ ) were derived from formula (1) using the $\mathrm{N}_{2}$-arginine and the $C_{1} D_{2^{-}}$ citrulline isotope, respectively. The tracer infused (in $\mu \mathrm{mol} \cdot \mathrm{kg} \mathrm{bw}^{-1} \cdot \mathrm{min}^{-1}$ ) is represented 
by $I$ and divided by $T T R_{A}$, which is the tracer-to-tracee ratio in the arterial plasma (26). TTR values were corrected for background values.

$$
\text { (1) } \quad \mathrm{QRa}=1 / \mathrm{TTR}_{\mathrm{A}}
$$

The endogenous rate of $Q R_{A R G}$, assumed to reflect de novo L-arginine synthesis plus L-arginine derived from protein breakdown, was estimated by correcting the total $Q$ Prod ARG for the contribution of L-arginine infused. The contribution of intragastric L-arginine infusion was estimated from the difference in L-arginine production by the splanchnic area between the L-arginine-treated and untreated group. Formula (2) was used to calculate the rate of whole-body synthesis of $N O\left(Q_{N O}\right)$ based upon the plasma isotope transfer from $\mathrm{N}_{2}$-arginine to $\mathrm{N}_{1}$-citrulline.

(2) $\quad Q_{\text {NO }}=Q_{\text {RaT }} *\left(T_{T R} R_{\text {N1-CIT }} / T_{T R}\right.$ N2-ARG $)$

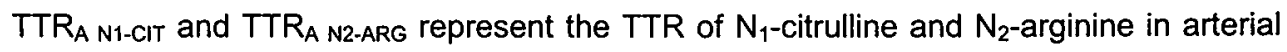
plasma, respectively.

The portal-drained viscera is defined as the total of all portal-drained organs which, besides for the spleen, stomach and pancreas, largely represents the intestines. The splanchnic area is the sum of the portal-drained viscera and liver, so that liver values could be obtained by subtracting portal-drained viscera from splanchnic values. Renal kinetics were calculated per two kidneys. L-arginine and NO metabolism across the hindquarter, the portal-drained viscera, the splanchnic region and the kidneys were calculated in a two-compartment model as was described previously (26).

The plasma flow rate $\left(\mathrm{mL} \cdot \mathrm{kg} b \mathrm{w}^{-1} \cdot \mathrm{min}^{-1}\right)$ across liver, portal-drained viscera and hindquarter was calculated using formula (1) based on the principle of indicator dilution methods in which I represents the rate of the $\mathrm{PAH}$ infused $\left(\mathrm{nmol} \cdot \mathrm{kg} \mathrm{bw}^{-1} \cdot \mathrm{min}^{-1}\right)$ near the organ. $[\mathrm{PAH}]_{V}$ and $[\mathrm{PAH}]_{\mathrm{A}}$ represent the concentration $(\mu \mathrm{mol} / \mathrm{L})$ of $\mathrm{PAH}$ in the venous and arterial blood, respectively. The venous $[\mathrm{PAH}]$ dilution depends on the organ blood flow rate. By sampling upstream and downstream of the organ, at the arterial and venous site, respectively, the organ blood flow can be calculated in (3). The blood flow was converted to plasma flow by using equation (4).

(3) Flow $_{\text {BLOOD }}=1 /\left([\mathrm{PAH}]_{V}-[\mathrm{PAH}]_{\mathrm{A}}\right)$

(4) FlOW $_{\text {PLASMA }}=$ flow $_{\text {BLOOD }} * 100 /(100-$ hematocrit $)$

Amino acid net balance was defined as NB (nmolekg $\left.\mathrm{bw}^{-1} \cdot \mathrm{min}^{-1}\right)$ and calculated according to formula (5) by multiplying the mean plasma flow by the difference between $[\mathrm{V}]$ and $[\mathrm{A}]$ that represent the venous and arterial plasma concentration of the amino acid ( $\mu \mathrm{mol} / \mathrm{L})$, respectively. Therefore, a negative NB represents net influx of amino acid into, and a positive NB, net efflux of amino acid from the organ. 


$$
\text { (5) } \mathrm{NB}=\text { flow } *([\mathrm{~V}]-[\mathrm{A}])
$$

In the fed pigs, the L-arginine net balance across the portal-drained viscera is corrected for the non-disposed L-arginine derived from enteral nutrition escaping disposal (incorporation into protein plus degradation) in the portal-drained viscera, appearing in the portal vein (2).

The rate of NO synthesis by individual organs (Organ No) can be calculated from conversion of $\mathrm{N}_{2}$-arginine to $\mathrm{N}_{1}$-citrulline across that organ. The term nb $\mathrm{N1}_{1-\mathrm{CIT}}$ represents the tracer net balance of the $\mathrm{N}_{1}$-citrulline isotope in arterial and venous plasma, respectively. Because of possible fractional extraction of citrulline and, therefore, $N_{1}$ citrulline $\left(F E_{\mathrm{CIT}}\right.$ ) by the organ, the $\mathrm{nb} \mathrm{N1}_{1} \mathrm{ClT}$ must be corrected for the loss of $\mathrm{N}_{1}$-citrulline tracer across the organ, which is possible by using the $\mathrm{C}_{1} \mathrm{D}_{2}$-citrulline isotope in equation (6). The term (1 - FE) represents the fraction of total amino acid that bypasses metabolism in the organ and so appears in the output and is used for correction of the $\mathrm{N}_{1}$-citrulline $\mathrm{nb}(7)$. The organ Syn No is derived from formula (8), in which TTR $\mathrm{A}$ N2-ARG represents the arterial $\mathrm{N}_{2}$-arginine enrichment. The TTR of $\mathrm{N}_{2}$-arginine in the arterial plasma free pool was assumed to be the same as the TTR of $\mathrm{N}_{2}$-arginine in the direct precursor pool of NO synthesis.

(6) $\quad \mathrm{FE} \mathrm{CIT}=\left\{\left(\mathrm{TTR}_{\mathrm{A}} *[\mathrm{~A}]-\mathrm{TTR}_{\mathrm{V}} *[\mathrm{~N}]\right) / \mathrm{TTR}_{\mathrm{A}} *[\mathrm{~A}]\right\}_{\mathrm{C102}-\mathrm{CIT}}$

(7) $\quad \mathrm{nb}_{\mathrm{N} 1-\mathrm{CIT}}=$ flow $^{*}\left\{[\mathrm{~V}] * \operatorname{TTR}_{\mathrm{V}}-[\mathrm{A}] * \operatorname{TTR}_{\mathrm{A}} *\left(1-\mathrm{FE}_{\mathrm{CIT}}\right)\right\}_{\mathrm{N1}-\mathrm{CIT}}$

(8) Organ $_{\mathrm{NO}}=\mathrm{nb}_{\mathrm{N1}-\mathrm{CIT}} / \mathrm{TTR}_{\mathrm{AN2}-\mathrm{ARG}}$

\section{Statistics}

Results are presented as means \pm SEM. Results at Day -1 and Day 0 between the two groups were very similar and, for that reason, pooled into one control group. The data were analyzed by a two-factor repeated measures ANOVA, using as within factor time with 4 levels (repeated measures: day $-1,0,1,2)$ and as between-factors treatment $(+1-$ $\mathrm{L}$-arginine treatment). Error alpha values are reported in tables in which $\mathrm{P}_{\mathrm{T}}$ indicates the significance of repeated measures over time (effect fasting plus endotoxin infusion), $P_{T \times G}$ the significance of interaction of repeated measures and treatment (time $x$ L-arginine treatment) and $P_{G}$ the significance of group effects ( $+/$ - L-arginine treatment). In case time effects were observed, the non-parametric 2 related samples test (Wilcoxon) was performed within the control group to test for differences between days: 1. to assess the effect of 8-h overnight fasting (Day 0 vs. Day -1) and 2. to assess the effect of fasting plus endotoxin intervention in time (Day 1 vs. Day 0 or Day 2 vs. Day -1). Additionally within the control group, data of temperature and MAP at 4-h and 8-h timepoints at Day 0 were compared with $0 \mathrm{~h}$ at Day 0 . Levels of significance were set at $P<0.05$. 


\section{Results}

Plasma flow: $\quad$ As shown in Table 1, the plasma flow across the liver, hindquarter and kidneys did not change in time. Although overall time effect for the portal-drained viscera plasma flow was present, no significant effect of 8 -h overnight fasting (Day 0 vs. 1) or the 24-h fasting endotoxin infusion period was detected (Day 1 vs. 0 and Day 2 vs. 1). L-Arginine intervention also did not significantly affect the organ plasma flow.

Table 1: Plasma flow in pigs at Day -1: during feeding, Day 0: after 8-h overnight fasting, Day 1: during 24-h fasting with endotoxin infusion and Day 2: during feeding and recovery from endotoxin infusion. Pigs received L-Ala (CON) or L-Arg (ARG) intervention.

\begin{tabular}{lccccccccc}
\hline & Day -1 & Day 0 & \multicolumn{2}{c}{ Day 1 } & \multicolumn{2}{c}{ Day 2 } \\
\hline & FED & FASTING & \multicolumn{2}{c}{ FASTING + ENDOTOXIN } & FED + POSTENDOTOXIN & \multicolumn{2}{c}{ ANOVA } \\
\hline CON & CON & CON & ARG & CON & ARG & P $_{\text {T }}$ & P $_{\text {TXG }}$ & PG $_{\text {G }}$ \\
\hline PDV & $39 \pm 3$ & $36 \pm 3$ & $39 \pm 4$ & $42 \pm 6$ & $48 \pm 7$ & $46 \pm 5$ & .03 & .70 & .68 \\
Liver & $59 \pm 8$ & $64 \pm 9$ & $66 \pm 11$ & $59 \pm 8$ & $55 \pm 20$ & $54 \pm 7$ & .84 & .26 & .76 \\
Hindquarter & $28 \pm 2$ & $34 \pm 5$ & $33 \pm 2$ & $40 \pm 8$ & $33 \pm 7$ & $41 \pm 8$ & .18 & .20 & .53 \\
Kidneys & $34 \pm 4$ & $54 \pm 7$ & $68 \pm 16$ & $45 \pm 11$ & $48 \pm 9$ & $31 \pm 4$ & .42 & .78 & .12 \\
\hline
\end{tabular}

Data are mean $\pm \mathrm{SEM}$ in $\mathrm{mL} \cdot \mathrm{kg} \mathrm{bw}^{-1} \cdot \mathrm{min}^{-1}$. Abbreviation: portal-drained viscera (PDV). Statistics by repeated measures ANOVA: a significant time effect $\left(P_{T}=0.03\right)$ was observed for PDV plasma flow.

Temperature, mean arterial pressure and blood gas values: The body temperature was elevated at 4 and $8 \mathrm{~h}$ of endotoxin infusion within the control group and was still elevated at $24 \mathrm{~h}$ as compared to $\mathrm{O}$ h as is shown in Figure 2A. Supplementary L-arginine did not influence the body temperature. A small, but significant transient increase in mean arterial blood pressure (MAP) was observed at $4 \mathrm{~h}$ of endotoxin infusion (Figure 2B), stabilizing again at $8 \mathrm{~h}$ (not significantly different from $0 \mathrm{~h}$ ). L-Arginine administration in endotoxin-treated pigs resulted in significantly reduced MAP $\left(P_{T X G}=0.01\right)$ at Day 1 and 2. All arterial blood gas values except for $\mathrm{pH}$ decreased upon 8-h overnight fasting as shown in Table 2. The endotoxin treatment plus fasting period within the control group resulted in decreased hematocrit and increased arterial $\mathrm{HCO}_{3}{ }^{-}$at Day 2 compared to Day -1. Arterial $\mathrm{PaCO}_{2}$ significantly decreased $\left(\mathrm{PG}_{\mathrm{G}}=0.01\right)$ and $\mathrm{HCO}_{3}^{-}$almost decreased $\left(P_{G}=0.06\right)$ with $L$-arginine infusion.

Arterial concentrations: The arterial concentrations of arginine and ornithine were significantly decreased after 8-h overnight fasting (Table 3 ) and further decreased upon sustained fasting plus endotoxin infusion. Endotoxin infusion plus fasting also reduced the $\mathrm{NO}_{x}$ concentration. Arginine, ornithine and urea concentrations were all higher $\left(P_{G}=0.00\right)$ in L-arginine-supplemented pigs whereas the $\mathrm{NO}_{X}$ concentration was not different $\left(P_{T X G}=0.09\right)$ compared with control pigs. 
Table 2: Hematocrit and blood gas values in pigs at Day -1: during feeding, Day 0 : after 8 -h overnight fasting, Day 1: during 24-h fasting with endotoxin infusion and Day 2: during feeding and recovery from endotoxin infusion. Pigs received L-Ala (CON) or L-Arg (ARG) intervention.

\begin{tabular}{|c|c|c|c|c|c|c|c|c|c|}
\hline & Day -1 & Day 0 & Day & y 1 & $\mathrm{Da}$ & & & & \\
\hline & FED & FASTING & FASTING + E & ENDOTOXIN & $F E D+P O S T$ & ENDOTOXIN & & ANOVA & \\
\hline & $\mathrm{CON}$ & $\mathrm{CON}$ & $\mathrm{CON}$ & ARG & $\mathrm{CON}$ & ARG & $P_{T}$ & $P_{T \times G}$ & $P_{G}$ \\
\hline $\mathrm{Ht}$ & $27.2 \pm 0.9$ & $24.0 \pm 0.6^{A A}$ & $23.1 \pm 1.5$ & $22.9 \pm 0.8$ & $21.5 \pm 1.2^{B}$ & $21.8 \pm 1.1$ & .00 & .47 & .38 \\
\hline $\mathrm{pH}$ & $7.41 \pm 0.01$ & $7.43 \pm 0.01$ & $7.46 \pm 0.01$ & $7.45 \pm 0.01$ & $7.45 \pm 0.01$ & $7.46 \pm 0.02$ & .04 & .74 & .73 \\
\hline $\mathrm{PaCO}_{2}$ & $46 \pm 1$ & $42 \pm 1^{A A}$ & $40 \pm 1^{B}$ & $34 \pm 1$ & $49 \pm 1$ & $44 \pm 1$ & .00 & .57 & .01 \\
\hline $\mathrm{PaO}_{2}$ & $99 \pm 3$ & $93 \pm 1^{\mathrm{AA}}$ & $92 \pm 1$ & $99 \pm 4$ & $100 \pm 4$ & $99 \pm 2$ & .05 & .93 & .55 \\
\hline $\mathrm{HCO}_{3}{ }^{-}$ & $29.1 \pm 0.8$ & $26.7 \pm 0.9^{A}$ & $27.1 \pm 1.2$ & $21.8 \pm 1.5$ & $32.9 \pm 1.4^{B}$ & $29.8 \pm 1$ & .00 & .48 & .06 \\
\hline $\mathrm{SO}_{2}$ & $97.2 \pm 0.3$ & $96.9 \pm 0.3^{A A}$ & $96.9 \pm 0.3$ & $97.7 \pm 0.2$ & $97.7 \pm 0.3$ & $97.7 \pm 0.3$ & .05 & .24 & .61 \\
\hline
\end{tabular}

Data are mean \pm SEM. Abbreviations: hematocrit=Ht, arterial $\mathrm{PCO}_{2}=\mathrm{PaCO}_{2}$ (Torr), $\mathrm{PO}_{2}=\mathrm{PaO}_{2}$ (Torr), bicarbonate $=\mathrm{HCO}_{3}{ }^{\circ}(\mathrm{mmol} / \mathrm{L}), \mathrm{O}_{2}$ saturation $=\mathrm{SO}_{2}(\%)$. Statistics by repeated measures ANOVA. Overall time effect $\left(P_{T}<0.05\right)$ was observed for all parameters and arginine effect $\left(P_{G}=0.01\right)$ was observed for $\mathrm{PaCO}_{2}$. ${ }^{A A} \mathrm{P}<0.01,{ }^{A} \mathrm{P}<0.05$ comparing Day 0 to -1 control sub-groups (effect overnight fasting) and ${ }^{\mathrm{B}} \mathrm{P}<0.05$ comparing Day 1 to 0 control sub-groups (effect endotoxin intervention in time).

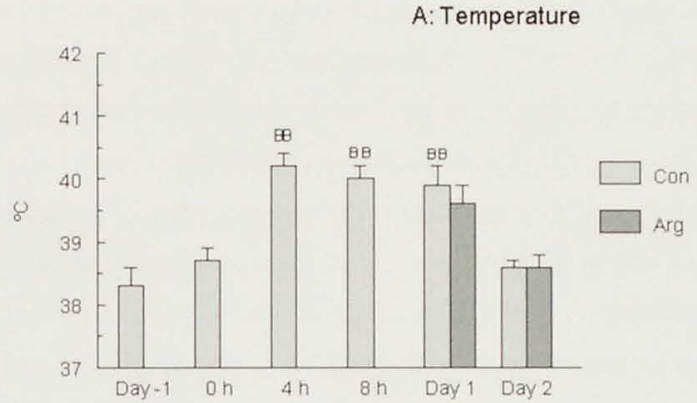

B: MAP

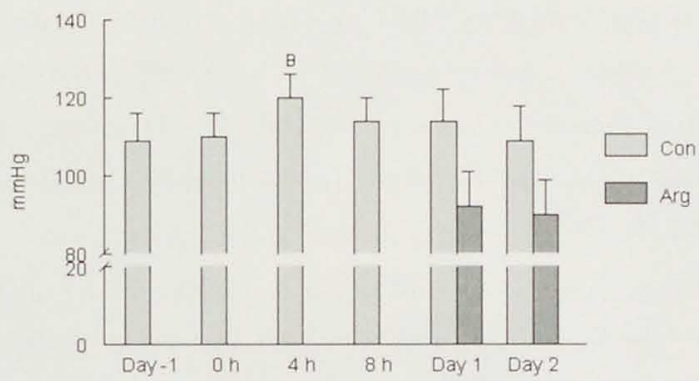

Figure 2 A: Body temperature and B: mean arterial pressure (MAP). Day -1 : during feeding, Day 0 : after 8-h overnight fasting, Day 1: during 24-h fasting with endotoxin infusion and Day 2: during feeding and recovery from endotoxin infusion. Pigs received L-Ala (CON) or L-Arg (ARG) intervention. Statistics by repeated measures ANOVA. A: overall time effect $\left(P_{T}=0.001\right)$ but no arginine effect for temperature was observed. ${ }^{\mathrm{BB}} \mathrm{P}<0.01$ comparing $4 \mathrm{~h}$ and $8 \mathrm{~h}$ to $0 \mathrm{~h}$ control subgroups (effect endotoxin intervention in time). B: overall time effect $\left(P_{T}=0.04\right)$ and time $x$ arginine effect $\left(P_{T X G}=0.01\right)$ for MAP was observed. ${ }^{8} \mathrm{P}<0.05$ comparing $4 \mathrm{~h}$ to $0 \mathrm{~h}$ control sub-groups (effect endotoxin intervention in time). 
Table 3: Arterial concentrations in pigs at Day -1: during feeding, Day 0: after 8-h overnight fasting, Day 1: during 24-h fasting with endotoxin infusion and Day 2: during feeding and recovery from endotoxin infusion. Pigs received L-Ala (CON) or L-Arg (ARG) intervention.

\begin{tabular}{|c|c|c|c|c|c|c|c|c|c|}
\hline & \multirow{2}{*}{$\frac{\text { Day -1 }}{\text { FED }}$} & \multirow{2}{*}{$\begin{array}{c}\text { Day } 0 \\
\text { FASTING }\end{array}$} & \multicolumn{2}{|c|}{ Day 1} & \multicolumn{2}{|c|}{ Day 2} & \multirow{2}{*}{\multicolumn{3}{|c|}{ ANOVA }} \\
\hline & & & FASTING + & ENDOTOXIN & FED + POST & ENDOTOXIN & & & \\
\hline & CON & CON & CON & ARG & CON & $A R G$ & $P_{T}$ & $P_{T \times G}$ & $P_{G}$ \\
\hline Arg & $86 \pm 6$ & $74 \pm 3^{\text {AA }}$ & $64 \pm 3^{B}$ & $465 \pm 20$ & $72 \pm 10$ & $359 \pm 46$ & .00 & .00 & .00 \\
\hline Orn & $98 \pm 7$ & $72 \pm 5^{A A}$ & $48 \pm 3^{88}$ & $562 \pm 26$ & $108 \pm 8$ & $337 \pm 18$ & .00 & .00 & .00 \\
\hline Urea & $3.7 \pm 0.3$ & $3.5 \pm 0.5$ & $2.7 \pm 0.2$ & $10.2 \pm 0.8$ & $3.2 \pm 0.4$ & $9.3 \pm 0.4$ & .00 & .00 & .00 \\
\hline $\mathrm{NO}_{x}$ & $49 \pm 10$ & $51 \pm 8$ & $20 \pm 7^{8}$ & $29 \pm 11$ & $31 \pm 10$ & $39 \pm 13$ & .03 & .09 & .40 \\
\hline
\end{tabular}

Data are mean $\pm S E M$ in $\mu \mathrm{mol} / \mathrm{L}$ except for urea in mmol/L. NO represents the sum of $\mathrm{NO}_{2}{ }^{-}$and $\mathrm{NO}_{3}{ }^{-}$. Statistics by repeated measures ANOVA; a significant time effect $\left(P_{T}<0.05\right)$ was observed for all parameters and both a group effect $\left(P_{G}<0.05\right)$ and time $x$ group interactions $\left(P_{T \times G}<0.05\right)$ were observed for Arg, Orn and urea concentrations. ${ }^{A A} P<0.01$ comparing Day 0 to -1 control sub-groups (effect overnight fasting) and ${ }^{\mathrm{BB}} \mathrm{P}<0.01,{ }^{\mathrm{B}} \mathrm{P}<0.05$ comparing Day 1 to 0 control sub-groups (effect endotoxin intervention in time).

Whole-body appearances: As shown in Figure 3A, the whole-body arginine appearance decreased $8 \mathrm{~h}$ after enteral nutrition was ceased within the control group but remained unchanged upon further fasting accompanied with endotoxin infusion. This whole-body arginine appearance increased as a result of L-arginine supplementation $\left(P_{G}=0.00\right)$. Intravenous $L$-arginine infusion at Day 1 endotoxin increased the arginine appearance 3-fold whereas intragastrically L-arginine at Day 2 postendotoxin increased arginine appearance 2 -fold. The total splanchnic arginine production between the intragastrically $L$-arginine-supplemented and $L$-alanine-supplemented pigs differed about $3.4 \mu \mathrm{mol} \cdot \mathrm{kg} \mathrm{bw}^{-1} \cdot \mathrm{min}^{-1}$, indicating a $65 \%$ recovery of the intragastrically infused Larginine in the circulation. The estimated endogenous production of arginine (total arginine appearance minus arginine infused) appeared reduced at both Day 1 and 2 when compared to controls.

The arterial $\mathrm{N}_{2}$-arginine to $\mathrm{N}_{1}$-citrulline conversion, representing whole-body synthesis of NO, dropped after 8-h food deprivation but remained unchanged after further fasting plus endotoxin challenge (Figure 3B). L-Arginine infusion increased whole-body synthesis of NO $\left(\mathrm{P}_{\mathrm{T} \times \mathrm{G}}=0.01\right)$. L-Arginine increased whole-body synthesis of NO 2.5-fold in the fasted endotoxemic animals and 1.7 -fold in the postendotoxemic condition. NO synthesis as percentage of L-arginine production under fasted, endotoxemic and fed, postendotoxemic conditions amounted to $10-20 \%$.

Hindquarter: Overnight 8-h fasting reduced the arginine influx or net uptake across the hindquarter (Figure 4A). Subsequent infusion with endotoxin and continuing fasting reversed the net uptake of arginine across the hindquarter into net release. When L-arginine was infused, arginine net uptake across the hindquarter tended to enhance 
$\left(P_{G}=0.11\right)$. The hindquarter $N O$ synthesis as measured by $N_{2}$-arginine to $N_{1}$-citrulline conversion is depicted in Figure 5A. No time effect on the hindquarter NO synthesis was observed. Although L-arginine treatment augmented the hindquarter NO synthesis about 7- to 8-fold during and after endotoxemia, respectively, this did not reach significance $\left(P_{T X G}=0.09\right)$ due to high variance in the $N_{1}$-citrulline tracer net balances across the hindquarter. L-arginine infusion did not change the $\mathrm{NO}_{x}$ efflux from the hindquarter at Day 1 , although an increasing trend was observed $\left(P_{G}=0.14\right.$, Figure $\left.6 A\right)$.

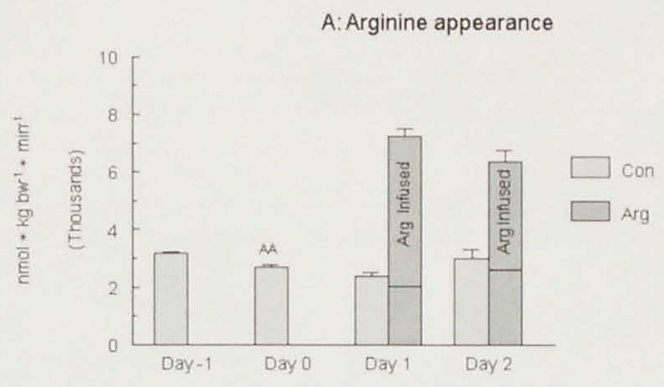

B: Nitric oxide synthesis

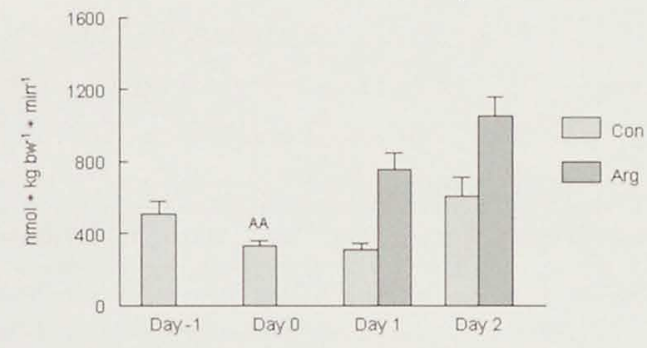

Figure 3 A: Whole-body arginine appearance and estimated L-Arg infusion (indicated in bar) and $\mathrm{B}$ : whole-body NO synthesis. Day 1 : during feeding, Day 0: after 8h overnight fasting, Day 1: during 24-h fasting with endotoxin infusion and Day 2: during feeding and recovery from endotoxin infusion. Pigs received L-Ala (CON) or L-Arg (ARG) intervention. Statistics by repeated measures ANOVA. A: overall time $\left(P_{T}=0.00\right)$, arginine $\left(P_{G}=0.00\right)$ and time $x$ arginine $\left(P_{T \times G}=0.00\right)$ effect was observed for whole-body arginine production. ${ }^{A A} \mathrm{P}<0.01$ comparing Day 0 to -1 control sub-groups (effect overnight fasting). B: overall time $\left(P_{T}=0.01\right)$, arginine $\left(P_{G}=0.01\right)$ and time $x$ arginine $\left(P_{T \times G}=0.01\right)$ effect was observed for whole-body NO synthesis. ${ }^{A A} \mathrm{P}<0.01$ comparing Day 0 to -1 control sub-groups (effect overnight fasting).

Portal-drained viscera: The arginine net balance across the portal-drained viscera was corrected for the arginine entering the portal circulation from enteral infusion as described in the materials and methods section. The net release of arginine was reduced after an 8-h overnight fast (Figure 4B), while an extra period of 24-h fasting plus endotoxin challenge resulted in a further reduction of arginine net release. L-Arginine supplementation time-dependently resulted in conversion of arginine efflux into influx across the portal-drained viscera $\left(P_{T X G}=0.00\right)$. Concomitantly, elevated arginine concentrations in jejunal mucosa biopsies were observed $\left(P_{G}=0.01\right.$, Figure 7$)$. No significant effect of 8-h fasting or endotoxin infusion plus fasting on the NO synthesis by the portal-drained viscera could be detected (Figure 5B). On the other hand, a significant increase in NO synthesis was achieved by L-arginine supplementation $\left(P_{T X G}=0.05\right)$. 
Moreover, L-arginine infusion tended to increase the $\mathrm{NO}_{\mathrm{x}}$ efflux from the portal-drained viscera at Day 1 , although not significantly ( $P G=0.12$, Figure $6 B$ ).
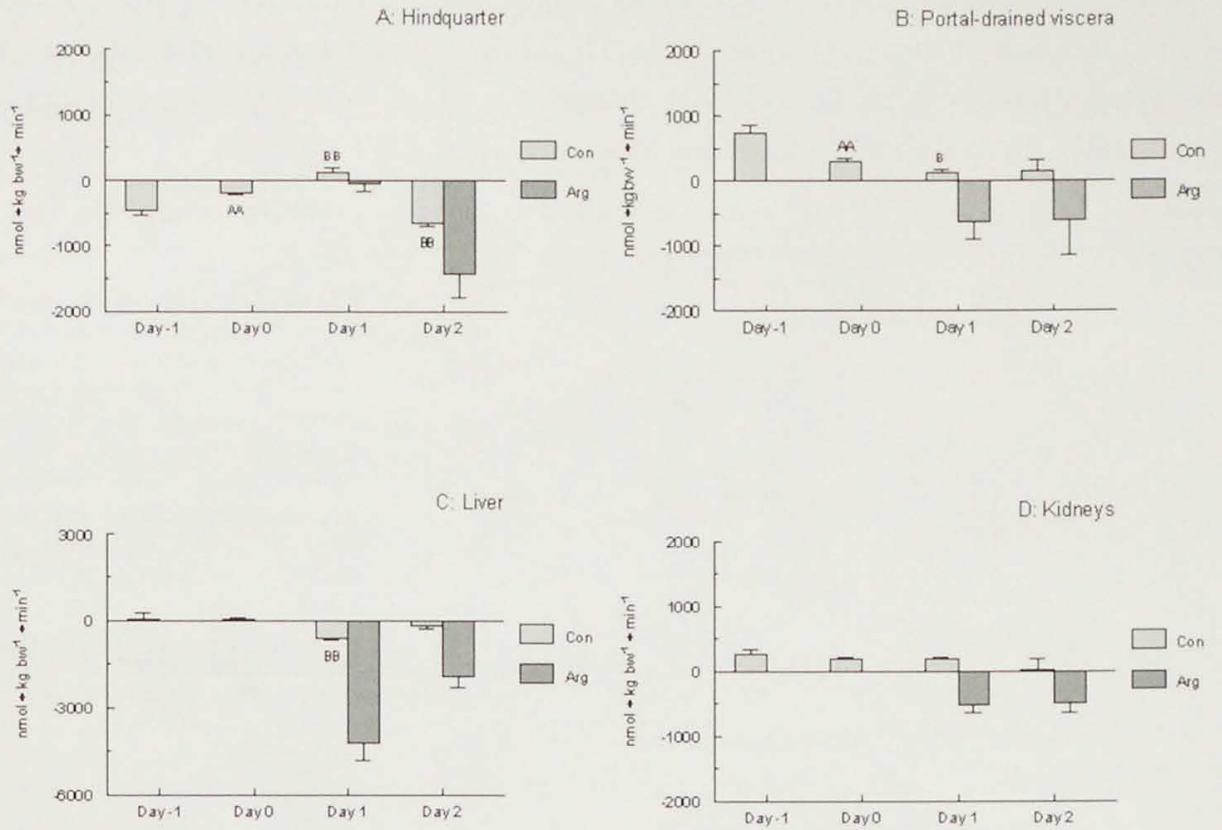

Figure 4. Net balance of arginine across $A$ : the hindquarter, B: the portal-drained viscera, C: the liver and D: the kidneys. Day -1: during feeding, Day 0: after 8-h overnight fasting, Day 1: during 24-h fasting with endotoxin infusion and Day 2: during feeding and recovery from endotoxin infusion. Pigs received L-Ala (CON) or L-Arg (ARG) intervention. Statistics by repeated measures ANOVA. A: overall time $\left(P_{T}=0.00\right)$ effect was observed for hindquarter arginine net balance. ${ }^{A A} \mathrm{P}<0.01$ comparing Day 0 to -1 control subgroups (effect overnight fasting) and ${ }^{\mathrm{BB}} \mathrm{P}<0.01$ comparing Day 1 to 0 control sub-groups (effect endotoxin intervention in time). $B$ : overall time $\left(P_{T}=0.00\right)$ and time $x$ arginine $\left(P_{T \times G}=0.00\right)$ effect was observed for portal-drained viscera arginine net balance. ${ }^{A} P<0.01$ comparing Day 0 to -1 control sub-groups (effect overnight fasting) and ${ }^{B} \mathrm{P}<0.05$ comparing Day 1 to 0 control sub-groups (effect endotoxin intervention in time). C: overall time $\left(P_{T}=0.00\right)$, arginine $\left(P_{G}=0.01\right)$ and time $x$ arginine $\left(P_{T \times G}=0.01\right)$ effect was observed for liver arginine net balance. ${ }^{B 8} \mathrm{P}<0.01$ comparing Day 1 to 0 control sub-groups (effect endotoxin intervention in time). $D$ : overall time $\left(P_{T}=0.00\right)$, arginine $\left(P_{G}=0.04\right)$ and time $x$ arginine $\left(P_{T \times G}=0.00\right)$ effect was observed for renal arginine net balance.

Liver: $\quad$ Under fed or fasted conditions the liver does not take up or release Larginine (Figure 4C). Endotoxin challenge in combination with fasting induced an influx of arginine into the liver. Treatment with $L$-arginine enhanced this arginine influx $\left(P_{G}=0.01\right.$ and $\mathrm{P}_{\mathrm{TXG}}=0.01$ ). Neither 8-h fasting, nor challenge with endotoxin plus fasting, changed the NO synthesis rate by the liver (Figure $5 \mathrm{C}$ ). Yet, administration of L-arginine was able to induce a time-related increase in hepatic NO synthesis compared to the endotoxemic control pigs $\left(P_{T X G}=0.05\right)$. Administration of $L$-arginine at Day 1 nevertheless exerted no 
significant effect on the $\mathrm{NO}_{x}$ efflux from the liver although a tendency to incline was present $\left(P_{G}=0.14\right.$, Figure $\left.6 C\right)$.

Kidneys: Infusion of endotoxin plus fasting did not affect the arginine net release from the kidneys (Figure 4D). Infusion with L-arginine resulted in a significant $\left(P_{G}=0.04\right.$ and $P_{T x G}=0.00$ ) net uptake of arginine into the kidneys. The fasting or endotoxin plus fasting did not significantly change the rate of NO synthesized by the kidneys. L-Arginine intervention, on the other hand, significantly increased it $\left(P_{T \times G}=0.05\right.$, Figure 5D). Moreover, L-arginine administration was almost able to promote renal $\mathrm{NO}_{\mathrm{x}}$ efflux $\left(P_{T X G}=0.06\right.$, Figure 6D).
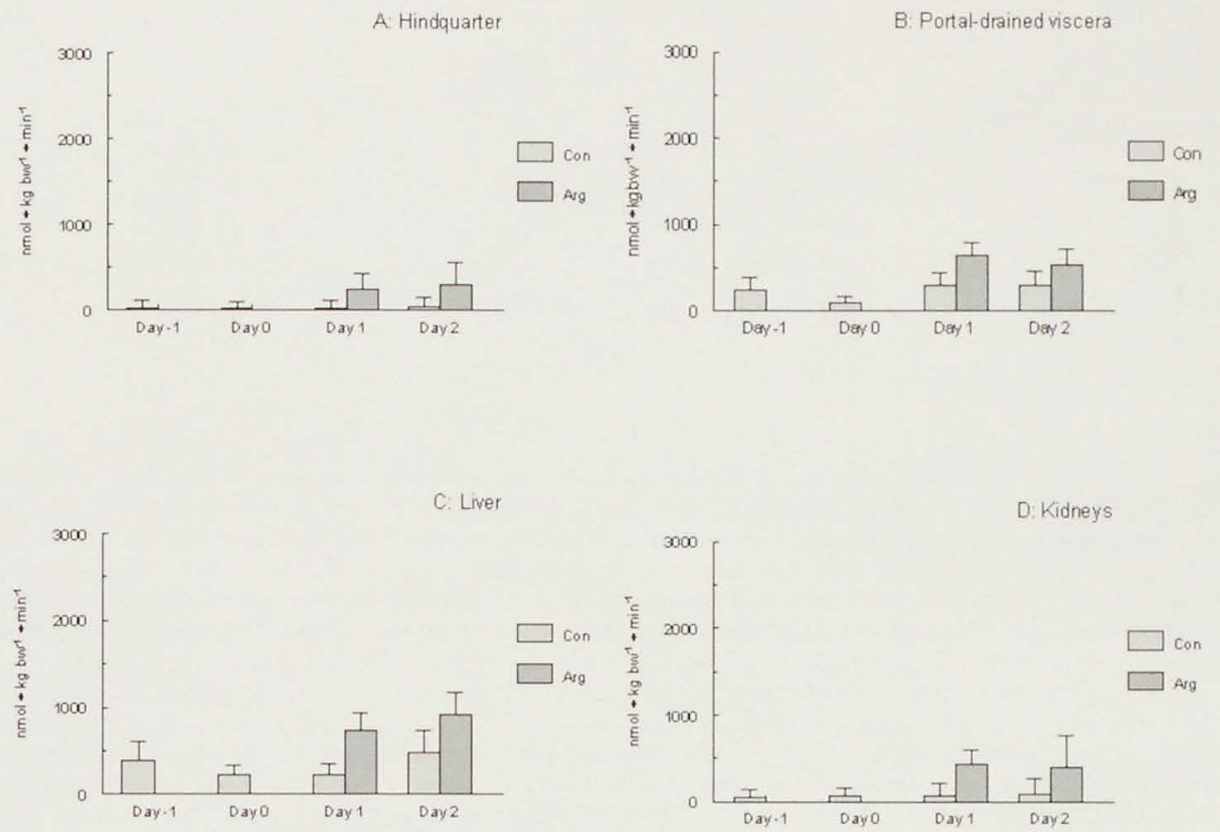

Figure $5 \mathrm{~A}$ : Synthesis of $\mathrm{NO}$ across the hindquarter, B: the portal-drained viscera, C: the liver and $\mathrm{D}$ : the kidneys. Day -1: during feeding, Day 0: after 8-h overnight fasting, Day 1: during 24-h fasting with endotoxin infusion and Day 2: during feeding and recovery from endotoxin infusion. Pigs received L-Ala (CON) or L$\operatorname{Arg}(A R G)$ intervention. Statistics by repeated measures ANOVA. A: no effect was observed for hindquarter NO synthesis. $B$ : overall time $\left(P_{T}=0.04\right)$ and overall time $x$ arginine $\left(P_{T \times G}=0.05\right)$ effect was observed for portal-drained viscera NO synthesis. C: overall time $\left(P_{T}=0.03\right)$ and overall time $x$ arginine $\left(P_{T \times G}=0.05\right)$ effect was observed for liver NO synthesis. $D$ : overall time $\left(P_{T}=0.04\right)$ and overall time $x$ arginine $\left(P_{T \times G}=0.05\right)$ effect was observed for kidney NO synthesis. 

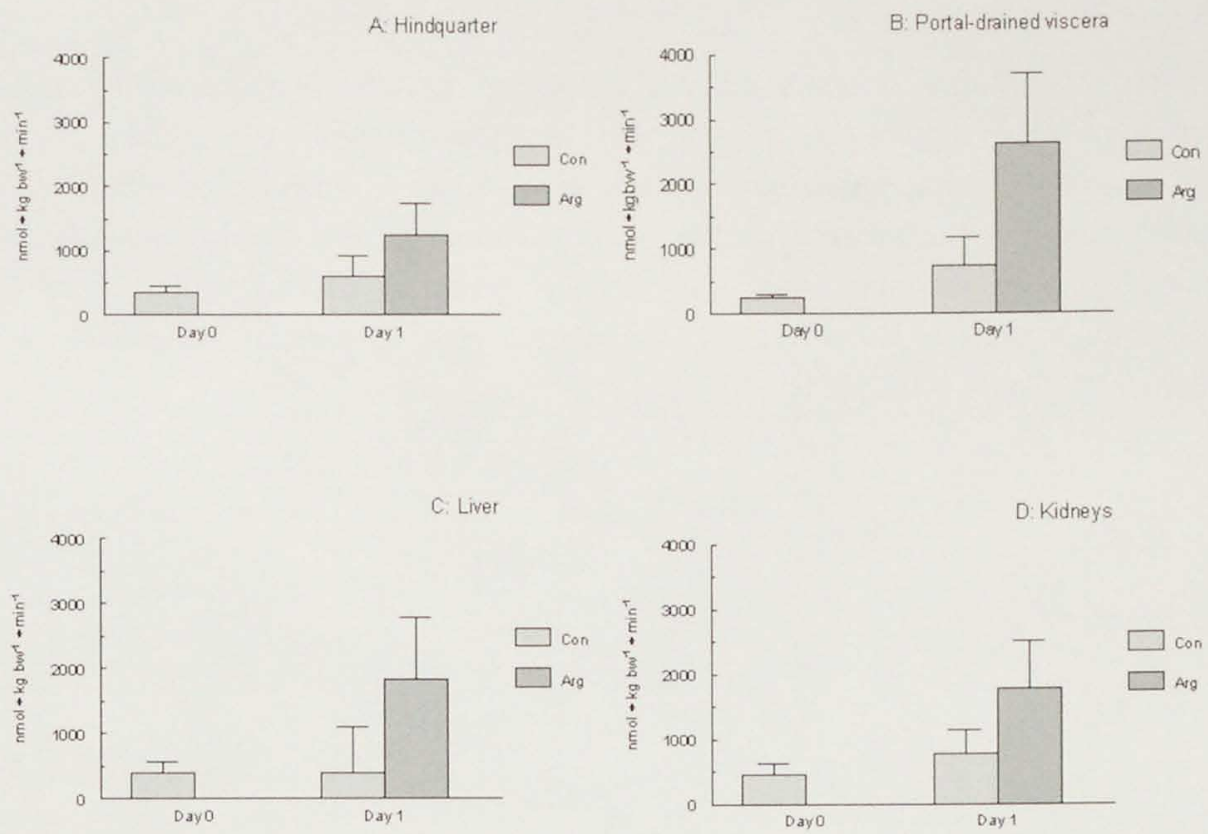

Figure $6 \mathrm{~A}$ : Flux of $\mathrm{NO}_{x}$ across the hindquarter, $\mathrm{B}$ : the portal-drained viscera, $\mathrm{C}$ : the liver and $\mathrm{D}$ : the kidneys. Day 0: after 8-h overnight fasting and Day 1: during 24-h fasting with endotoxin infusion. Pigs received $L-A l a(C O N)$ or $L-A r g(A R G)$ intervention. Statistics by repeated measures ANOVA. A: no effect was observed for hindquarter $\mathrm{NO}_{X}$ flux. $\mathrm{B}$ : overall time $\left(\mathrm{P}_{\mathrm{T}}=0.01\right)$ but no arginine effect was observed for portal-drained viscera $N O_{x}$ flux. C: overall time $\left(P_{T}=0.02\right)$ but no arginine effect was observed for liver $N O_{x}$ flux. D: overall time $\left(P_{T}=0.02\right)$ but no arginine effect was observed for kidney $\mathrm{NO}_{\mathrm{x}}$ flux.

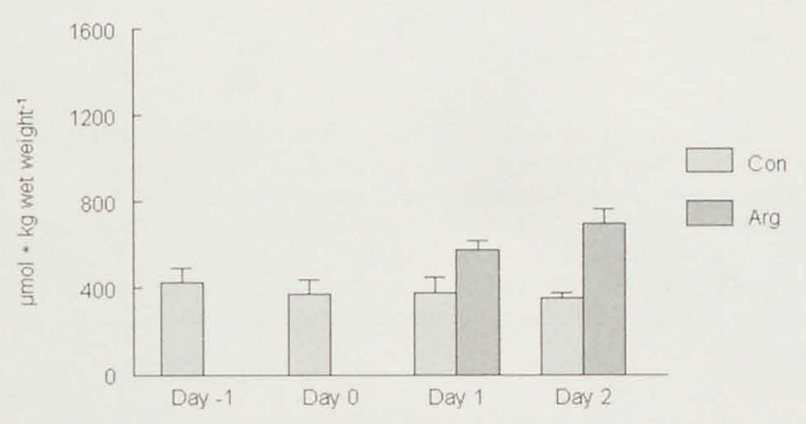

Figure 7. Tissue concentration of arginine in jejunal mucosa. Day -1 : during feeding, Day 0: after 8 -h overnight fasting, Day 1 : during 24 $\mathrm{h}$ fasting with endotoxin infusion and Day 2: during feeding and recovery from endotoxin infusion. Pigs received L-Ala $(\mathrm{CON})$ or $\mathrm{L}$ Arg (ARG) intervention. Statistics by repeated measures ANOVA. Overall arginine $\left(P_{G}=0.01\right)$ effect was observed. 


\section{Discussion}

We hypothesized that under conditions of chronic stress such as long-lasting endotoxemia, the availability of L-arginine may become limiting as a substrate for NO. Providing L-arginine under such circumstances may support organ NO formation by meeting the increased substrate demand. In order to investigate the effect of L-arginine supplementation during prolonged endotoxemia on the organ L-arginine metabolism including the role of L-arginine as a precursor in the NO-pathway, we measured the Larginine net balance and NO synthesis across the main organs by means of stable isotope techniques. Our hypothesis that supply of L-arginine could be of benefit in sustaining NO generation during and after endotoxemia is supported by the observation that in these conditions both intravenously and intragastrically administrated L-arginine enhanced the whole-body synthesis of NO.

In the present study we demonstrated that the response to endotoxin represented the development of a normotensive state including increased temperature and maintained arterial pressure and blood flow to the organs. The $20 \mathrm{mmHg}$ decline in arterial blood pressure that was observed during one day L-arginine infusion we suppose was attributable to a NO-mediated decrease in systemic vascular resistance $(25,27)$. Despite this modest hypodynamic effect of L-arginine, the body temperature of the pigs was not significantly affected by L-arginine. Although a NO-mediated dilatory action by L-arginine on regional blood flow might be expected $(12,15)$, L-arginine did not increase the organ perfusion. These results therefore create the premise that L-arginine only provokes some minor hemodynamic effects during and after endotoxin. Moreover, as decreased $\mathrm{PaCO}_{2}$ and nearly decreased arterial $\mathrm{HCO}_{3}{ }^{-}$show, L-arginine appears to be involved to some extent in compensated metabolic acidosis.

In this experiment, fasting exerted a decreasing effect on the rate of L-arginine appearing in plasma, whereas endotoxin infusion in combination with fasting appeared to exert no effect on the whole-body rate of L-arginine appearance. Previous experiments (unpublished results, Bruins MJ, 2001) showed that increased whole-body L-arginine appearance by endotoxin challenge was counterbalanced by decreased whole-body Larginine appearance by fasting. Remarkably, as was assessed by $\mathrm{N}_{2}$-arginine to $\mathrm{N}_{1}$ citrulline isotope conversion in plasma, the whole-body NO production also decreased upon fasting and remained unchanged upon fasting along endotoxin infusion. The rate of NO generated, therefore, seems related to the rate of L-arginine appearing in the circulation. The observation that whole-body NO synthesis during endotoxemia did not drop below basal synthesis rates present during fasting, indicates absence of an absolute L-arginine deficiency. This however may not exclude that L-arginine availability 
becomes rate limiting to sustain maximal rates of NO formation by the endotoxin-induced NOS-Il enzyme.

As we intended, the intravenously administrated L-arginine during endotoxemia in the fasting state increased L-arginine whole-body appearance 3-fold. Dietary L-arginine was only partially metabolized by the portaL-drained viscera and liver, $-33 \%$ by-passing splanchnic metabolism, comparable to findings in healthy humans fed an L-arginineenriched diet (4). Consequently, intragastrically provided L-arginine resulted in a 2 -fold increase in L-arginine whole-body appearance. Irrespective of the administration route, additional L-arginine under endotoxemia and recovery thereof, was capable of promoting the whole-body NO production implying that formation of NO is dependent on the Larginine rate of appearance in the circulation. The portal-drained viscera, the kidneys and, most pronounced the liver appeared to account for this upregulation in NO synthesis as measured by stable isotope conversion techniques but not by means of nitrite/nitrate fluxes. We cannot explain why the sum of NO synthesized by the organs exceeded the whole-body NO synthesis. Possibly, steady state conditions for the product of $\mathrm{N}_{2}$-arginine, $\mathrm{N}_{1}$-citrulline, were not entirely achieved thereby contributing to an underestimation of the whole-body appearance of $\mathrm{N}_{1}$-citrulline and an overestimation of the organ $\mathrm{N}_{1}$-citrulline appearance. Moreover, the present findings demonstrate that the increase in NO synthesized by the portal-drained viscera is strongly related to the increased intracellular L-arginine concentrations of jejunal mucosal tissue. Apparently, the NOS enzyme under endotoxemic conditions is not saturated with L-arginine and is able to respond to changes in intracellular L-arginine concentration. In the present study we demonstrate, by means of direct measurements, a stimulating effect of L-arginine supply under endotoxemic circumstances on NO production.

L-Arginine administration did not significantly affect the arterial nitrite/nitrate concentrations whereas it tended to promote the net nitrite/nitrate fluxes released from the organ. Arterial nitrite/nitrate concentration has been considered an unreliable indicator of NO synthesis since it may be confounded by denitrificating commensal anaerobes (21) but also by fluid shifts that vary with renal function (14). In addition to their high variation, the organ nitrite/nitrate fluxes were quantitatively incomparable to the $\mathrm{NO}$ synthesis rates as measured by $\mathrm{N}_{2}$-arginine to $\mathrm{N}_{1}$-citrulline conversion. Therefore, arterial nitrite/nitrate concentrations, but also organ nitrite/nitrate fluxes give poor indicative value of NO synthesis, and stable isotope measurements to quantify wholebody NO production are preferable. An accompanying advantage of the stable isotope technique is that, whereas nitrite/nitrate measurements can not be performed under fed conditions, it can be applied under both fasting and fed conditions. 
Additional L-arginine during endotoxemia induced a negative balance of L-arginine across the hindquarter, the portal-drained viscera, the liver and the kidneys, indicating Larginine net uptake by these organs. Besides decreased organ production, increased Larginine net uptake may represent elevated organ metabolism including accelerated Larginine degradation by the arginase and NOS pathways. The observation that L-arginine is released by the portal-drained viscera under both fasting and fed conditions implies that, besides the kidneys, the intestines of the pig are well capable of L-arginine biosynthesis due to presence of argininosuccinate synthase and lyase.

In this experiment, exogenous supplementation of L-arginine showed to be capable of supporting enhanced biosynthesis of NO both during hyperdynamic endotoxemia and recovery thereof. These findings indicate that the exogenous L-arginine constitutes a determining factor in the endogenous synthesis of NO under septic conditions, irrespective of the administration route. In this mild model of chronic endotoxemia, Larginine availability does not become a rate-limiting factor for basal rates of NO production. However, under more severe septic conditions, L-arginine availability may become insufficient to sustain minimal rates of NO synthesis and, under such circumstances, provide a therapeutic tool by means of upregulating organ and wholebody NO formation.

\section{Acknowledgments}

The authors wish to thank Mr. H.M.H. van Eijk and Mr. J.L.J.M. Scheijen for analytical amino acid measurements and Mrs. G.A.M. ten Have for assistance with operational procedures.

\section{References}

1. Beaumier, L., L. Castillo, A. M. Ajami, and V. $R$. Young. Urea cycle intermediate kinetics and nitrate excretion at normal and "therapeutic" intakes of arginine in humans. Am Physiol Soc 269: E884-E896, 1995.

2. Bruins, M. J., P. B. Soeters, and N. E. Deutz. Endotoxemia affects organ protein metabolism differently during prolonged feeding in pigs. $J$ Nutr 130: 3003-13., 2000.

3. Castillo, L., L. Beaumier, A. M. Ajami, and V. $R$. Young. Whole body nitric oxide synthesis in healthy men determined from [15N]-arginineto-[15N]citrulline labeling. Proc Natl Acad Sci USA 93: 11460-11465, 1996.

4. Castillo, L., T. E. Chapman, Y. M. Yu, A. Ajami, J. F. Burke, and V. R. Young. Dietary uptake by the splanchnic region in adult humans. Am J Physiol 265: E532-E539, 1993.
5. Cook, H. T., and V. Cattell. Role of nitric oxide in immune-mediated diseases. Clin Sai 91: 375-384, 1996.

6. Dejong, C. H., N. E. Deutz, and P. B. Soeters. Renal ammonia and glutamine metabolism during liver insufficiency- induced hyperammonemia in the rat. $J$ Clin Invest 92: 2834-40., 1993.

7. Dodds, W. J. The pig model for biomedical research. Federation Proc 41: 247-256, 1982.

8. Evoy, D., M. D. Lieberman, T. J. Fahey, 3rd, and J. M. Daly. Immunonutrition: the role of arginine. Nutrition 14: 611-7, 1998.

9. Fink, M. P., and S. O. Heard. Laboratory models of sepsis and septic shock. J Surg Res 49: 186-196, 1990. 
10. Freund, H., S. Atamian, J. Holroyde, and J. E. Fischer. Plasma amino acids as predictors of the severity and outcome of sepsis. Ann Surg 190: 571-6, 1979.

11. Hattori, Y., K. Kasai, and S. S. Gross. Cationic amino acid transporter gene expression in cultured vascular smooth muscle cells and in rats. Am J Physio/ 276: H2020-8, 1999.

12. Henderson, J. L., R. Statman, J. N. Cunningham, W. Cheng, P. Damiani, A. Siconolfi, and J. H. Horovitz. The effects of nitric oxide inhibition on regional hemodynamics during hyperdynamic endotoxemia. Arch Surg 129: 1271-1275, 1994.

13. Kelly, E., S. M. Morris, and T. R. Billiar. Nitric oxide, sepsis, and arginine metabolism. J Parenter Enteral Nutr 19: 234-238, 1995.

14. Kirkeboen, K. A., and O. A. Strand. The role of nitric oxide in sepsis-an overview. Acta Anaesthesiol Scand 43: 275-88, 1999.

15. Kreimeier, U., U. B. Brueckner, S. Gerspach, $\mathrm{K}$. Veitinger, and K. Messmer. A porcine model of hyperdynamic endotoxemia: pattern of respiratory, macrocirculatory, and regional blood flow changes. $J$ Invest Surg 6: 143-156, 1993.

16. Lorente, J. A., M. A. Delgado, C. Tejedor, E. Mon, M. Hervas, T. Pascual, P. FernandezSegoviano, G. Rieppi, A. Soler, D. Ayuso, and A. Esteban. Modulation of systemic hemodynamics by exogenous L-arginine in normal and bacteremic sheep. Crit Care Med 27: 2474-9, 1999.

17. Miller, E. R., and D. E. Ullrey. The pig as a model for human nutrition. Annu Rev Nutr 7: 361-82, 1987.

18. Moncada, S., R. M. J. Palmer, and E. A. Higgs. Nitric oxide: physiology, pathophysiology and pharmacology. Pharmacol Rev 13: 109-142, 1991.
19. Muscara, M. N., and J. L. Wallace. Nitric Oxide. V. therapeutic potential of nitric oxide donors and inhibitors. Am J Physiol 276: G1313-6, 1999.

20. Park, J.-H., S.-H. Chang, K.-M. Lee, and S.-H. Shin. Protective effect of nitric oxide in an endotoxin-induced septic shock. Am J Surg 171: 340-345, 1996.

21. Salzman, A. L., and L. Andrew. Nitric oxide in the gut. New Horizons 3: 352-364, 1995.

22. Ten Have, G. A. M., M. C. F. Bost, J. C. A. W. Suyk-Wierts, A. E. J. M. van den Bogaart, and N. E. P. Deutz. Simultaneous measurement of metabolic flux in portally-drained viscera, liver, spleen, kidney and hindquarter in the conscious pig. Lab Anim 30: 347-358, 1996.

23. Van Eijk, H. M. H., D. R. Rooyakkers, and N. E. P. Deutz. Automated determination of polyamines by high-performance liquid chromatography with simple sample preparation. $J$ Chromatogr 730: 115-120, 1996.

24. Van Eijk, H. M. H., D. R. Rooyakkers, and N. E. P. Deutz. Determination of amino acid isotope enrichment using liquid chromatography-mass spectrometry. Anal Biochem 271: 8-17, 1999.

25. Vromen, A., M. S. Arkovitz, B. Zingarelli, A. L. Salzman, V. F. Garcia, and C. Szabo. Lowlevel expression and limited role for the inducible isoform of nitric oxide synthase in the vascular hyporeactivity and mortality associated with cecal ligation and puncture in the rat. Shock 6: 248-53, 1996.

26. Wolfe, R. R. Radioactive and stable isotope tracers in biomedicine. Principles and practice of kinetic analysis. New York: Wiley-Liss, 1992.

27. Yu, Y. M., J. F. Burke, R. G. Tompkins, R. Martin, and V. R. Young. Quantitative effects of interorgan relationships among arginine and citrulline metabolism. Am J Physiol 271: E1098-E1109, 1996. 


\title{
EFFECTS OF ARGININE SUPPLEMENTATION ON ORGAN UREA CYCLE INTERMEDIATE METABOLISM IN ENDOTOXEMIC PIGS
}

\author{
Maaike J Bruins \\ Wouter H Lamers ${ }^{*}$ \\ Alfred J Meijer * \\ Nicolaas EP Deutz
}

Department of Surgery, Maastricht University, The Netherlands

- Departments of Anatomy \& Embryology (WHL) and Biochemistry (AJM), University of Amsterdam, AMC, The Netherlands 


\section{Abstract}

Arginine supplementation is one of the therapeutic strategies under disease conditions. Increased arginine levels may resemble the metabolic condition as occurs with arginase deficiency. This study allows to assess the consequences of high levels of arginine for on whole body and organ fluxes of urea cycle intermediates under disease conditions.

At Day 1 conscious pigs were challenged with $E$. coli endotoxin for 24-h followed 8-h later by intravenous arginine infusion $(n=8)$ whereas controls $(n=6)$ received alanine. After cessation of endotoxin infusion at $24 \mathrm{~h}$, pigs received arginine or alanine-enriched intragastric nutrition (Day 2). Stable isotope protocols enabled measurements of amino acid and urea whole-body and organ (hindquarter muscle, portal-drained viscera, liver and kidneys) fluxes at Day 1 and 2. Jejunal biopsies provided intracellular amino acid concentrations.

Arginine infusion at endotoxemia Day 1 and post-endotoxemia Day 2 increased the whole-body urea appearance, mostly accounted for by the liver. Arginine decreased the visceral net glutamine uptake at Days 1 and 2 and citrulline synthesis at Day 1 in parallel with reduced glutamine and citrulline concentrations in gut tissue. Reduced renal citrulline disposal and citrulline-to-arginine recycling was observed during arginine treatment at Day 1 and 2 accounting for the reduced whole-body citrulline turnover and arginine de novo synthesis.

Not only increased arginine degradation but also decreased arginine de novo synthesis, although to a lesser extent, regulate elevated levels of arginine during endotoxemia. Arginine homeostasis therefore appears achieved by adaptive changes in both arginine degradation and endogenous synthesis.

\section{Introduction}

Arginine participates in two major metabolic pathways including its incorporation into protein and its conversion to urea and ornithine catalyzed by arginase. Arginine also serves as precusor in the synthesis of polyamines via ornithine by ornithine decarboxylase (ODC), of creatine and of nitric oxide (NO) by NO synthase (NOS). The flux through the urea cycle and NOS pathway appears to be accelerated by inflammatory stimuli such as endotoxin and cytokines $(21,25,30)$. Increased arginine utilization during severe prolonged sepsis may participate in the depletion of plasma arginine levels as frequently observed under septicemic conditions $(10,20,24)$.

Arginine supplementation during sepsis has been implicated as a therapeutic modality to meet elevated requirements. An increasing number of studies provide support for the 
benefit of the supplemental use of arginine under conditions such as severe sepsis and postoperative stress. Previous studies suggest that the nitrogen-retaining property of arginine might be of benefit in catabolic conditions such as severe sepsis and postoperative stress (for review: (13)). Moreover, arginine showed great potential as an immunomodulator under stress conditions such as sepsis. However, high levels of arginine resulting from intravenous or enteral supplementation may evoke major metabolic changes, especially those of the urea cycle intermediates. The capacity of arginase greatly exceeds the normal rates of arginine produced and the liver will largely deal with high arginine levels by increasing the arginine conversion to urea. The manner in which the gut, the muscle and kidney deal with high arginine supply is, however, unknown. Similar as to patients receiving arginine-supplemented diets, the metabolic alterations evoked by high arginine levels may resemble the metabolic alterations that occur with hereditary argininemia owing to arginase deficiency. The arginine which accumulates in these patients may lead to eventual alternative yet unknown pathways of arginine metabolism.

The body arginine homeostasis is regulated by interplay of the different organs, which simultaneously produce and metabolize arginine. Previously, the effect of arginineenriched diets on whole body changes in arginine, ornithine and NO metabolism was explored in healthy humans by the use of stable isotopes (5). In view of the fact that arginine is supplied as a therapeutic intervention in a clinical setting to injured patients, we explored the effect of increasing plasma arginine levels on whole body arginine, citrulline and urea appearance rates under endotoxemic and post-endotoxemic conditions. In addition, catheter implantation together with stable isotope infusion enabled us to study the effect of elevated arginine levels on urea cycle intermediate fluxes across the individual organs (portal-drained viscera, the liver, the hindquarter muscle and the kidney). As a model of hyperdynamic sepsis (14), pigs were continuously infused low doses of endotoxin and were resuscitated with high amounts of fluid. To mimic the clinical situation, arginine was administered either by the intravenous route during fasting endotoxemia, or via the intragastric route as supplement in the diet under postendotoxemic conditions.

\section{Materials and methods}

\section{Animals}

Pigs were offsprings of Yorkshire $x$ Dutch Landrace. Three-month old pigs, weighting 20$22 \mathrm{~kg}$ body weight were individually housed. Pigs were given $1 \mathrm{~kg}$ of regular pig feed (149 g crude protein $/ \mathrm{kg}$; Landbouwbelang, Roermond, The Netherlands; $16 \%$ crude 
protein) daily, which supported a growth rate of approximately 300 gram per day. Before surgery, pigs were randomly assigned to one of the two treatment groups. The Animal Ethics Committee of the Maastricht University approved the study.

\section{Surgical Procedure}

The night before surgery, food was withheld from pigs. One hour after premedication with $10 \mathrm{mg} / \mathrm{kg}$ body weight (bw) intramuscular azoperone (Stresnil, Janssen Pharmaceutica, Beersse, Belgium), anesthesia was induced by a mixture of $\mathrm{N}_{2} \mathrm{O} / \mathrm{O}_{2}(1: 2)$ and halothane $(0.8 \%)$. After intubation, the pigs were intravenously administered $6.25 \mathrm{mg} / \mathrm{kg}$ bw lincomycin.2 $\mathrm{HCl}$ (Lincomycin, A.U.V., Cuyk, The Netherlands) as bactericidal prophylaxis and $12.5 \mathrm{mg} / \mathrm{kg}$ bw spectinomycin. $\mathrm{HCl}$ lyophil (Spectinomycin, A.U.V.) as bacteriostatic prophylaxis. To avoid coagulation and as postoperative analgesia, $50 \mathrm{mg} / \mathrm{kg}$ bw flunixine (Finadyne, Schering-Ploegh, Brussel, Belgium) was given. During surgery, the anesthesia was maintained by the $\mathrm{N}_{2} \mathrm{O} / \mathrm{O}_{2}$-halothane mixture and i.v. Lactetrol (per $\mathrm{mL}$ : $5.76 \mathrm{mg} \mathrm{NaCl}, 0.37 \mathrm{mg} \mathrm{KCl}, 0.37 \mathrm{mg} \mathrm{CaCl}_{2}, 0.2 \mathrm{mg} \mathrm{MgCl}_{2}, 5 \mathrm{mg}$ sodium lactate, methyl parahydroxy bezoicacid, Janssen Pharmaceutica). The surgical procedure has been described in detail elsewhere (26). In brief, after a midline laparotomy was performed, eight catheters were inserted in the blood vessels. Two catheters were placed in the abdominal aorta; just above the bifurcation (A1) and just above the right renal vein (A2), and two in the inferior caval vein at the corresponding positions (V1 and V2, respectively). Furthermore, catheters were placed into the portal $(P)$, hepatic $(H)$, renal $(R)$, and splenic (S) veins. The $A 1$ and the $S$ catheters were used for infusion of paraaminohippuric acid (PAH), the $\mathrm{V} 2$ catheter for infusion of isotopes and endotoxin and the $A 2, P, H, V 1$ and $R$ catheters for blood sampling across the portal-drained viscera, the splanchnic area, the hindquarter and the kidney, respectively. A gastrostomy catheter was inserted into the stomach. All catheters were tunneled through the abdominal wall and skin. The pigs also underwent construction of a Bishop-Koop stoma. Briefly, $20 \mathrm{~cm}$ distal to the ligament of Treitz, the jejunum was transected and the anatomical continuity of the remaining bowel reestablished with a single-layer running suture (PDS 4-0 synthetic polydioxanon; Ethicon, Norderstedt, Germany) according to an end-to-side anastomosis. During recovery of the pigs, an inflated balloon catheter (size Ch.16, 3-5 $\mathrm{ml}$; Rüsch-Gold, Kernen, Germany) served to close the stoma and avoid leakage from the jejunum.

\section{Postoperative care}

The pigs wore a canvas harness to protect the catheters and stoma, and to allow easy handling. Postoperative care of the pigs was standard (26). To avoid catheter tip infections and to maintain patency, catheters were regularly flushed with $0.9 \% \mathrm{NaCl}$ 
(saline) and filled with a solution containing a mixture of gentamycin ( $20 \mathrm{~g} / \mathrm{L}$, Gentamycin 5\%, A.U.V., Cuyk, The Netherlands) and chymotrypsin (0.225 U/L, Chymotrypsin, Merck, Darmstadt, Germany) in saline. During handling, the pigs were placed in a movable cage to get accustomed to this condition.

\section{Experimental protocol}

Pigs recovered from the operation within 14 days. Before starting the experiments, pigs were accustomed to a high-protein liquid ready-to-use enteral diet ( $37.5 \mathrm{mg}$ protein per $\mathrm{mL}$, Nutrison Steriflo Protein-Plus, Nutricia, Zoetermeer, The Netherlands). For that purpose, the enteral diet was continuously infused via a swivel system into their gastrostomy catheter. The enteral diet was infused at a rate of $4 \mathrm{~mL} \cdot \mathrm{kg} \mathrm{bw}-1 \cdot \mathrm{h}-1(\sim 150 \mathrm{~g}$ protein / day) for 3 days of which the first day the diet was infused at half rate in addition to $500 \mathrm{~g}$ of their regular pig feed to get pigs accustomed to the enteral nutrition. After an 8-h overnight fast, experiments were started. An infusion of endotoxin (Escherichia coli lipopolysaccharide 055:B5, Sigma Chemicals Co., St. Louis, MO, USA), dissolved in saline, was started at a rate of $3 \mu \mathrm{g} \cdot \mathrm{kg} \mathrm{bw}^{-1} \cdot \mathrm{h}^{-1}$ via the venous (V2) catheter. Endotoxin was infused for a period of $24 \mathrm{~h}$. Simultaneously, pigs were resuscitated with $20 \mathrm{~mL} \cdot \mathrm{kg}$ $b w^{-1} \cdot h^{-1}$ saline infusion to replenish intravascular volume losses. Pigs received either an iso-osmolar infusion of L-arginine (Arg group, $\mathrm{n}=8,5.3 \mu \mathrm{mol} \bullet \mathrm{kg} \mathrm{bw}^{-1} \bullet \mathrm{min}^{-1}, \mathrm{pH} 7.3$ ) or Lalanine as a control amino acid at an isocaloric rate (Con group, $n=6,10.6 \mu \mathrm{mol} \bullet \mathrm{kg} \mathrm{bw}^{-}$ ${ }^{1} \cdot \mathrm{min}^{-1}, \mathrm{pH}$ 7.3). At $24 \mathrm{~h}$ of endotoxin and L-arginine/L-alanine infusion, blood was sampled from the A2, P, H, V1 and R catheters. Also at 24-h, mucosal tissue biopsies were taken via the jejunal stoma. Immediately thereafter, endotoxin and saline infusions

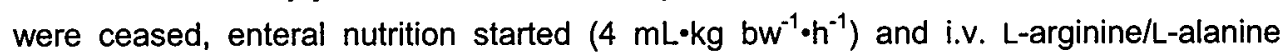
infusion continued by intragastric infusion at a similar infusion rate. Again, $24 \mathrm{~h}$ after feeding and cessation of endotoxin, blood samples and jejunal tissue biopsies were obtained. Temperature of the pigs was monitored during the entire experimental protocol.

\section{Isotope infusion protocol}

Blood was sampled at Day 1 and 2 when steady state conditions for PAH and stable isotopes were reached. Steady state conditions for PAH and isotopes were assessed in a pilot experiment in which blood samples were collected in triplicate at 10-min intervals. A plateau was reached when concentrations (PAH) or tracer-to-tracee ratios (isotopes) at different blood sampling sites no longer increased with time. One hour after start of the infusions, steady state conditions for PAH and stable isotopes were obtained $(26,28)$.

Background blood samples were taken before performing the infusion protocol. The infusion protocol (26) was started by giving a prime of PAH $(25 \mathrm{mmol} / \mathrm{L}, \mathrm{A} 1422$, Sigma Chemicals Co., St. Louis, MO, USA). Directly thereafter, PAH infusion was started at a 
constant rate of $40 \mathrm{~mL} / \mathrm{h}$ per catheter through the $\mathrm{S}$ and the A1 catheter. Simultaneously, a prime followed by a constant infusion of the stable isotopes was administered via the V2 catheter. The prime of tracer was calculated on basis of the body pool size of tracee whereas the infusion rate was calculated on basis of body appearance rate. The primed infusion of isotopes consisted of L-[guanidino- ${ }^{15} \mathrm{~N}_{2}$ ]arginine $\left(\mathrm{N}_{2}\right.$-arginine; prime: 3.2

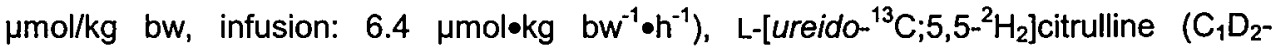
citrulline; prime: $1.5 \mu \mathrm{mol} / \mathrm{kg}$ bw, infusion: $0.6 \mu$ moløkg bw $\left.{ }^{-1} \bullet \mathrm{h}^{-1}\right)$ and $\left[{ }^{13} \mathrm{C}\right]$ urea $\left({ }^{13} \mathrm{C}\right.$-urea; prime: $54 \mu \mathrm{mol} / \mathrm{kg}$ bw, infusion: $\left.6.0 \mu \mathrm{mol} \bullet \mathrm{kg} \mathrm{bw}^{-1} \bullet \mathrm{h}^{-1}\right) . N_{2}$-arginine, $C_{1} D_{2}$-citrulline and ${ }^{13} \mathrm{C}$-urea were purchased from Mass Trace (Woburn, MA, USA). Blood was collected during steady state, one hour after start of the primed infusions.

\section{Sample processing}

Directly after sampling, blood was apportioned in pre-chilled, heparinized tubes (Sarstedt, Nümbrecht, Germany). After centrifugation at $4^{\circ} \mathrm{C}$ for $5 \mathrm{~min}$ at $8,500 \mathrm{~g}$, plasma was collected and kept on ice. For amino acid concentration and isotopic enrichment analysis, $500 \mu \mathrm{L}$ of plasma was deproteinized by mixing with $20 \mathrm{mg}$ dry sulfosalicylic acid. To determine ammonia and urea concentrations, $400 \mu \mathrm{L}$ plasma was mixed with 40 $\mu \mathrm{L}$ of $3.5 \mathrm{~mol} / \mathrm{L}$ trichloroacetic acid (TCA; Merck, Darmstadt, Germany) solution. For PAH determination, $300 \mu \mathrm{L}$ of whole blood was added to $600 \mu \mathrm{L}$ of $0.7 \mathrm{mmol} / \mathrm{L}$ TCA solution, thoroughly mixed and centrifuged to collect the supernatant. Tissue biopsies of the jejunum were collected on ice and rinsed in saline. Tissue was deproteinized by adding approximately $30 \mathrm{mg}$ tissue to $400 \mu \mathrm{L} 5 \%$ SSA for amino acid and polyamine determinations, and to $400 \mu \mathrm{L} 10 \%$ TCA for ammonia determinations. Subsequently, tissue was homogenized by beating for $30 \mathrm{~s}$ with $300 \mathrm{mg}$ glass beads (Biospeck, Bartlesville, OK, USA) in a Mini-Beadbeater (Biospeck). The homogenate was centrifuged at $11,000 \mathrm{~g}$ at $4^{\circ} \mathrm{C}$ for $10 \mathrm{~min}$ and supernatant collected. All plasma and tissue samples were frozen in liquid nitrogen and stored at $-80^{\circ} \mathrm{C}$ until use. Tissue biopsies of the jejunum were collected on ice and rinsed in saline. All plasma and tissue samples were frozen in liquid nitrogen and stored at $-80^{\circ} \mathrm{C}$ until use.

\section{Biochemical analysis}

Whole blood PAH and plasma ammonia concentrations were detected spectrophotometrically on an automated Cobas Mira-S analysis system (Hoffmann-La Roche, Basel, Switzerland) by standard enzymatic methods, using commercially available kits (9). Ammonia concentration was also measured on a Cobas Mira-S based on the ammonia to glutamate conversion method, measuring NADPH extinction (9). Concentrations and tracer-to-tracee ratios (TTR) of amino acids and urea were determined by a fully automated liquid chromatography-mass spectrometry (LC-MS, 
Thermoquest LCQ, Veenendaal, The Netherlands) system after pre-column derivatization with o-phthaldialdehyde (28). Polyamine concentrations were determined by HPLC as has been described before (27).

\section{Calculations of whole-body and organ arginine and citrulline kinetics}

The whole-body rate of appearance ( $\mathrm{Q} \mathrm{Ra}$ in $\left.\mu \mathrm{mol} \cdot \mathrm{kg} \mathrm{bw}^{-1} \cdot \mathrm{min}^{-1}\right)$ of arginine, citrulline and urea were derived from formula (1) using the $N_{2}$-arginine, the $C_{1} D_{2}$-citrulline and the ${ }^{13} \mathrm{C}$-urea isotope, respectively. The tracer infused (in $\mu \mathrm{mol} \cdot \mathrm{kg} \mathrm{bw}^{-1} \cdot \mathrm{min}^{-1}$ ) is represented by $I$ and divided by $T T R_{A}$, which is the tracer-to-tracee ratio in the arterial plasma (33). TTR values were corrected for background values. In steady-state conditions, the rate of appearance into the plasma compartment equals the rate of disappearance leaving this compartment.

\section{(1) $\quad \mathrm{QRa}=\mid / \mathrm{TTR}_{\mathrm{A}}$}

When arginine is exogenously administered, the endogenous of arginine appearance rate is the difference between total arginine appearance rate ( $Q R a$ ARG) and arginine appearing from either intravenous or the intragastric infusion. The contribution of intragastric arginine infusion was estimated from the difference in arginine appearance by the splanchnic area between the arginine-treated and control group. The whole body de novo synthesis of arginine from citrulline $\left(Q_{\mathrm{CIT} \rightarrow A R G}\right)$ was calculated by the following formula (2) in which TTR $\mathrm{A}_{\text {C1D2-ARG }}$ and TTR $\mathrm{A}_{\mathrm{A} \text { C1D2-CIT }}$ are the tracer-to-tracee ratios of $\mathrm{C}_{1} \mathrm{D}_{2}$-arginine and $\mathrm{C}_{1} \mathrm{D}_{2}$-citrulline in arterial plasma, respectively.

$$
Q_{C I T \rightarrow A R G}=Q_{\text {Ra }} \text { ARG } *\left(T T R_{A C 1 D 2-A R G} / T T R_{A \text { C1D2-CIT }}\right)
$$

The portal-drained viscera are defined as the total of all portal-drained organs which, in addition to the spleen and pancreas, largely represents the intestines. The splanchnic area is the sum of the portal-drained viscera and liver, so that liver values can be obtained by subtracting portal-drained viscera from splanchnic values. Renal kinetics was calculated per two kidneys. Arginine, citrulline and urea metabolism across the hindquarter, the portal-drained viscera, the splanchnic region and the kidneys were calculated in a two-compartment model as described previously (33).

The plasma flow rate $\left(\mathrm{mL} \cdot \mathrm{kg} \mathrm{bw}^{-1} \cdot \mathrm{min}^{-1}\right)$ across the organs was calculated using formula (3) and (4) based on the principle of indicator dilution methods in which I represents the rate of the $\mathrm{PAH}$ infused near the organ between the arterial and venous sampling site (26). $[\mathrm{PAH}]_{v}$ and $[\mathrm{PAH}]_{\mathrm{A}}$ represent the concentration of $\mathrm{PAH}$ in the venous and arterial blood of the organ, respectively. The venous $\mathrm{PAH}$ dilution depends on the organ blood flow rate. By sampling at the arterial and venous site of the organ, the organ blood flow can be calculated in (3). The blood flow was converted to plasma flow using equation (4). 


\section{(3) Flow $_{\text {BLOOD }}=1 /\left([\mathrm{PAH}]_{\mathrm{V}}-[\mathrm{PAH}]_{\mathrm{A}}\right)$}

(4)

$$
\text { FloW PLASMA }=\text { FlOW }_{\text {BLOOD }} * 100 /(100-\text { hematocrit })
$$

Substrate net balance was defined as NB $\left(\mathrm{nmol} \cdot \mathrm{kg} \mathrm{bw}^{-1} \cdot \mathrm{min}^{-1}\right)$ and calculated according to formula (5) by multiplying the mean plasma flow with difference between venous and arterial plasma concentration across the organ, $[\mathrm{V}]$ and $[\mathrm{A}]$, respectively. Therefore, a positive NB represents net efflux from, and a negative NB, net influx into the organ.

(5) $\mathrm{NB}=$ flow $*([\mathrm{~V}]-[\mathrm{A}])$

Organ disposal rate of arginine, citrulline and urea was calculated using the net balance ( $\mathrm{nb}, \mathrm{nmol} \cdot \mathrm{kg} \mathrm{bW}^{-1} \cdot \mathrm{min}^{-1}$ ) of the $\mathrm{N}_{2}$-arginine, $\mathrm{C}_{1} \mathrm{D}_{2}$-citrulline and ${ }^{13} \mathrm{C}$-urea stable isotopes, respectively, across the organ. The disposal rate of arginine by an organ represents the total rate metabolized; conversion into other metabolites plus incorporation into protein. The renal urea disposal represents disappearance by excretion. Since citrulline is not incorporated into protein, the renal citrulline disposal represents conversion into arginine. To calculate arginine, citrulline or urea disposal, tracer nb was calculated in (6) based on $A-V$ tracer concentration differences. TTR $R_{A}$ and TTR represent the arterial and venous tracer-to-tracee ratio in plasma. Disposal was calculated as in (7). Since the NB of a substrate across an organ is the net difference between production and disposal of that substrate, the production can be calculated in (8).

$$
\left.\mathrm{nb}=\text { flow } *\left\{\left([\mathrm{~A}] * \operatorname{TTR}_{\mathrm{A}}\right)-(\mathrm{M}] * \operatorname{TTR}_{\mathrm{V}}\right)\right\}
$$

(7) Disposal $=\mathrm{nb} / \mathrm{TTR}_{\mathrm{A}}$

(8) Production $=$ Disposal + NB

During enteral feeding, the arginine produced by the portal-drained viscera is a combination of endogenous arginine production and arginine derived from the nutrition escaping visceral metabolism. Endogenous arginine production by the portal-drained viscera was calculated by correcting total arginine production for the contribution of nondisposed arginine entering the portal circulation from enteral infusion (4). Also, visceral amino acid net balances were corrected.

The organ de novo synthesis of arginine can be calculated from conversion of $C_{1} D_{2-}$ citrulline to $C_{1} D_{2}$-arginine, using the $n b$ of $C_{1} D_{2}$-arginine. Because of possible disposal of $C_{1} D_{2}$-arginine in the organ, the tracer $\mathrm{nb}$ is calculated as in (6) but corrected for the loss by fractional extraction across the organ. The organ de novo arginine synthesis ( $O$

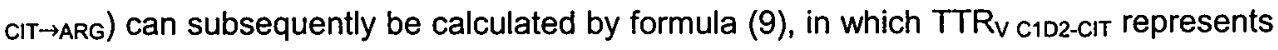
the venous $C_{1} D_{2}$-citrulline enrichment.

$$
\text { (9) } \mathrm{O}_{\text {CIT } \rightarrow \text { ARG }}=\mathrm{nb} \text { C1D2-ARG } / T_{T R R_{V}} \text { C1D2-CIT }
$$




\section{Statistics}

Results are presented as means \pm SEM. The Mann-Whitney $U$ test was performed for group comparisons at Day 1 and Day 2. Levels of significance were set at $P<0.05$.

\section{Results}

\section{Whole-body parameters}

The body temperature of the pigs was not affected by arginine treatment, neither during, nor after endotoxin infusion (Table 1). The arterial $\mathrm{pH}$ also was not significantly different between the arginine and control group at Day 1 (Table 1). In the intravenously argininetreated endotoxemic pigs, the arterial concentrations of arginine, ornithine and urea were significantly higher, whereas the arterial concentrations of citrulline, glutamine and ammonia were lower as compared to endotoxemic controls (Table 1). Intragastric arginine infusion in the recovery phase of endotoxemia mediated the same effect.

Table 1: Body temperature, arterial $\mathrm{pH}$ and arterial concentrations.

\begin{tabular}{|c|c|c|c|c|c|c|c|}
\hline & \multicolumn{6}{|c|}{ Day 1} & \multirow[b]{3}{*}{$\mathrm{P}$} \\
\hline & \multicolumn{6}{|c|}{ FASTING ENDOTOXEMIA } & \\
\hline & \multicolumn{3}{|c|}{ Con } & \multicolumn{3}{|c|}{ Arg } & \\
\hline Temperature $\left({ }^{\circ} \mathrm{C}\right)$ & 39.9 & \pm & 0.3 & 39.6 & \pm & 0.4 & \\
\hline $\mathrm{pH}$ & 7.46 & \pm & 0.01 & 7.45 & \pm & 0.01 & \\
\hline $\mathrm{HCO}_{3}^{-}$ & 27.1 & \pm & 1.2 & 21.8 & \pm & 1.5 & \\
\hline Arginine & 64 & \pm & 3 & 465 & \pm & 20 & ** \\
\hline Ornithine & 48 & \pm & 3 & 562 & \pm & 26 & ** \\
\hline Citrulline & 36 & \pm & 4 & 25 & \pm & 1 & ** \\
\hline Glutamine & 233 & \pm & 15 & 187 & \pm & 13 & $\star$ \\
\hline $\mathrm{NH}_{3}$ & 78 & \pm & 9 & 68 & \pm & 11 & \\
\hline Urea & 2.1 & \pm & 0.2 & 3.3 & \pm & 0.7 & ** \\
\hline
\end{tabular}

\begin{tabular}{rcc}
\hline \multicolumn{5}{c}{ Day 2} \\
\hline \multicolumn{1}{c}{ CED POST-ENDOTOXEM1A } \\
\hline $38.6 \pm 0.1$ & $38.6 \pm 0.2$ \\
$7.46 \pm 0.01$ & $7.45 \pm 0.02$ \\
$32.9 \pm 1.4$ & $29.8 \pm 1.0$ \\
$72 \pm 10$ & $359 \pm 46$ \\
$108 \pm 8$ & $337 \pm 18$ \\
$69 \pm 5$ & $55 \pm 3$ \\
$689 \pm 65$ & $325 \pm 19$ \\
$116 \pm 23$ & $89 \pm 22$ \\
$2.5 \pm 0.4$ & $3.9 \pm 0.6$
\end{tabular}

Abbreviations: $\mathrm{NH}_{3}=$ ammonia, $\mathrm{HCO}_{3}{ }^{-}=$bicarbonate. Endotoxemia: at $24-\mathrm{h}$ endotoxin infusion during fasting. Pigs received i.v. L-Ala (Con) or L-Arg (Arg) infusion. Post-endotoxemia: 24-h after cessation of endotoxin infusion and start of enteral feeding. Pigs received L-Ala or L-Arg intragastrically. Con group; $n=6$, Arg group, $n=8$. Data are mean $\pm S E M$. Concentrations in $n m o l / L$, except for urea $(\mu$ mol $/ L)$. Statistics by Mann-Whitney $U:{ }^{\star} P<0.05,{ }^{*} P<0.01$; significant difference between the groups at Day 1 or 2 . Complete list of amino acids available on request. 
A. Arginine appearance

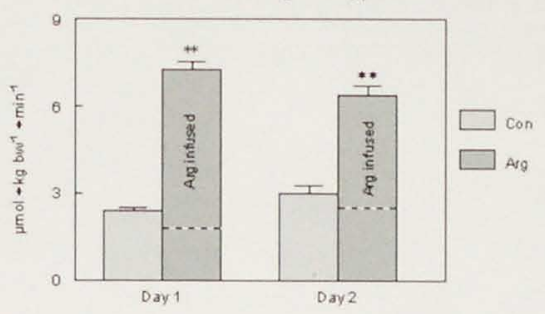

B: Arginine de novo synthesis

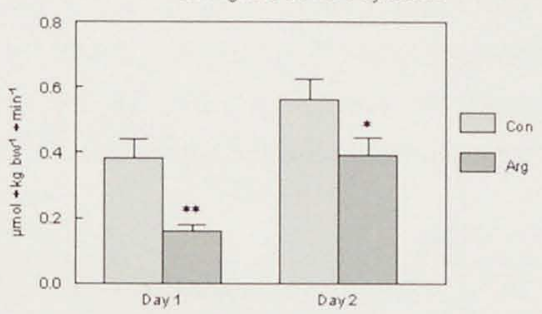

C. Citrulline appearance

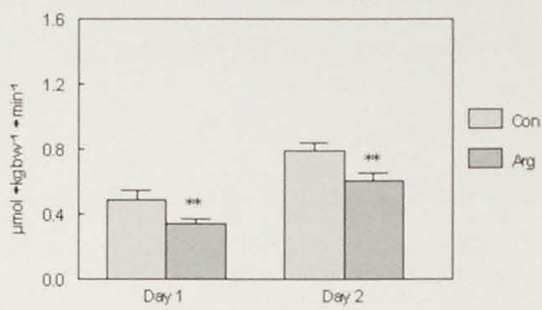

D: Urea appearance

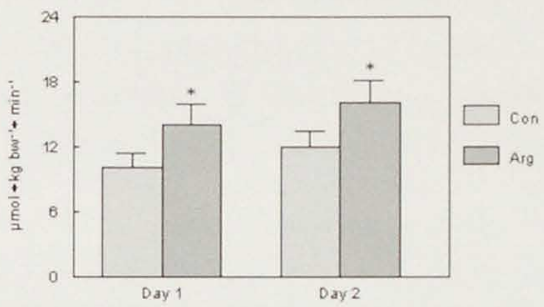

Figure 1. Whole-body appearance rate of (A) arginine, (B) de novo synthesized arginine, (C) citrulline and (D) urea in pigs at Day 1 during endotoxin infusion receiving L-Ala (Con) or L-Arg (Arg) intravenously and at Day 2 during recovery from endotoxin infusion receiving L-Ala or L-Arg intragastrically. A: estimated arginine infusion rate indicated in bar. Statistics by Mann-Whitney $U$ : ${ }^{\star} P<0.05,{ }^{\star \star} P<0.01$, significant difference between the groups at Day 1 or 2 .

Intravenous L-arginine infusion along with endotoxin infusion increased the arginine whole-body appearance rate 3 -fold as shown in Figure 1A. One day after endotoxin treatment, the splanchnic arginine production (data not shown) between the intragastric arginine-supplemented and control group differed about $3.4 \mu \mathrm{mol} \cdot \mathrm{kg} \mathrm{bw}^{-1} \cdot \mathrm{min}^{-1}$. This indicates that $65 \%$ of the intragastrically provided arginine $\left(5 \mu \mathrm{mol} \cdot \mathrm{kg} \mathrm{bw}^{-1} \cdot \mathrm{min}^{-1}\right)$ recovers in the circulation resulting in a 2 -fold increase in whole-body arginine appearance. Isotopic conversion of $C_{1} D_{2}$-citrulline to $C_{1} D_{2}$-arginine in plasma revealed that the whole body de novo synthesized arginine from citrulline decreased when Larginine was supplemented (Figure 1B). Moreover, L-arginine supplementation significantly reduced the whole-body citrulline appearance in both endotoxemic and postendotoxemic pigs (Figure $1 \mathrm{C}$ ). The percentage de novo synthesized arginine from endogenously appearing arginine in the Con group amounted 16 and $20 \%$ in fasted and fed pigs, respectively. The rate of citrulline appearance exceeded the citrulline to arginine conversion rate under both conditions, indicating that the rate of citrulline appearing or disappearing from the whole-body compartment was not equivalent to the arginine de 
novo synthesized. As can be seen in Figure 1D, both i.v. and the intragastrically infused L-arginine increased the whole body urea appearance indicating increased urea production.

\section{Organ parameters}

The plasma flow across the organs is presented in Table 2. The organ plasma flows did not change in response to L-arginine intervention, neither during endotoxin challenge, nor the following day. The amino acid, ammonia and urea net balance or flux rates across the organs that were estimated from plasma flow times arterial and venous plasma concentration differences are shown in Figure 2. The, by means of stable isotope measurements, estimated arginine and citrulline disposal and production rates, are summarized in Table 3 and 4, respectively. The arginine de novo synthesis rate is shown in Table 5 and urea disposal (excretion into urine) and production rate across the kidneys is shown in Table 6.

Table 2: Plasma flow across the organs.

\begin{tabular}{lccc}
\hline & \multicolumn{3}{c}{ Day 1} \\
\cline { 1 - 3 } & \multicolumn{3}{c}{ FASTING ENDOTOXEMIA } \\
\cline { 1 - 3 } Hindquarter & $33 \pm 2$ & $40 \pm 8$ \\
\cline { 3 - 4 } PDV & $39 \pm 4$ & $42 \pm 6$ \\
Liver & $66 \pm 11$ & $59 \pm 8$ \\
Kidneys & $68 \pm 16$ & $45 \pm 11$
\end{tabular}

\begin{tabular}{ccc}
\hline \multicolumn{4}{c}{ Day 2 } \\
\hline \multicolumn{3}{c}{ Arg } \\
\hline Con POST-ENDOTOXEMIA \\
\hline $3 \pm 7$ & $41 \pm 8$ \\
$48 \pm 7$ & $46 \pm 5$ \\
$55 \pm 20$ & $54 \pm 7$ \\
$48 \pm 9$ & $31 \pm 4$ \\
\hline
\end{tabular}

Abbreviations: PDV $=$ portal-drained viscera. Pigs at Day 1 during endotoxin infusion received L-Ala (Con) or L-Arg (Arg) intravenously and at Day 2 during recovery from endotoxin infusion received L-Ala or L-Arg intragastrically. Con group; $n=6$, Arg group, $n=8$. Data are mean $\pm S E M$ in $\mathrm{mL} \cdot \mathrm{kg} \mathrm{bw}^{-1} \cdot \mathrm{min}^{-1}$. Statistics by Mann-Whitney U: ${ }^{*} P<0.05,{ }^{* *} P<0.01$; significant difference between the groups at Day 1 or 2 .

Hindquarter: Intravenous arginine supplementation during 24-h endotoxin infusion at Day $1(P=0.06)$ and intragastric arginine infusion the following day induced influx of arginine into the hindquarter area (Figure 2A). Arginine disposal increased to a higher extent than the arginine production at Day 1 and Day 2 during arginine administration (Table 3). No citrulline net balance, disposal or production could be detected across the hindquarter during or after endotoxin challenge (Table 4). Also at Day 1 and Day 2, the de novo synthesis of arginine in the hindquarter area measured from conversion of $C_{1} D_{2}-$ arginine out of $C_{1} D_{2}$-citrulline was not significantly different from zero (Table 5). Also, ammonia and urea fluxes were not significantly different from zero. Arginine infusion at Day 1 and Day 2 had no effect on the glutamine net efflux from the hindquarter but significantly induced ornithine net influx in both the endotoxemic and postendotoxemic groups compared to controls (Figure 2A). 
Table 3: Disposal and production of arginine by the organs.

\begin{tabular}{|c|c|c|c|c|c|}
\hline & & \multicolumn{4}{|c|}{ Day 1} \\
\hline & & \multicolumn{4}{|c|}{ FASTING ENDOTOXEMIA } \\
\hline & & \multicolumn{2}{|c|}{ Con } & Arg & $P$ \\
\hline \multirow[t]{2}{*}{ Hindquarter } & Disposal & $0.5 \pm$ & $=0.2$ & $4.1 \pm 2.1$ & ** \\
\hline & Production & $0.6 \pm$ & 0.2 & $4.1 \pm 2.2$ & $* *$ \\
\hline \multirow[t]{2}{*}{ PDV } & Disposal & $0.4 \pm$ & 0.2 & $4.8 \pm 1.0$ & ** \\
\hline & Production & $0.6 \pm$ & $=0.2$ & $4.2 \pm 0.8$ & ** \\
\hline \multirow[t]{2}{*}{ Liver } & Disposal & $1.3 \pm$ & $=0.3$ & $6.5 \pm 1.0$ & ** \\
\hline & Production & $0.7 \pm$ & 0.3 & $2.2 \pm 0.5$ & $\star$ \\
\hline \multirow[t]{2}{*}{ Kidney } & Disposal & $0.1 \pm$ & 0.1 & $1.9 \pm 0.2$ & ** \\
\hline & Production & $0.3 \pm$ & 0.1 & $1.4 \pm 0.2$ & ** \\
\hline
\end{tabular}

\begin{tabular}{cccc}
\hline \multicolumn{4}{c}{ Day 2 } \\
\hline \multicolumn{3}{c}{ FED POST-ENDOTOXEMIA } \\
\hline \multicolumn{2}{c}{ Con Arg } & $P$ \\
\hline $1.1 \pm 0.3$ & $3.9 \pm 0.9$ & $* *$ \\
$0.4 \pm 0.2$ & $2.4 \pm 0.7$ \\
$1.0 \pm 0.3$ & $1.9 \pm 0.4$ \\
$1.2 \pm 0.3$ & $1.3 \pm 0.8$ \\
$0.9 \pm 0.3$ & $3.5 \pm 0.3$ \\
$0.7 \pm 0.8$ & $1.5 \pm 0.3$ \\
$0.7 \pm 0.2$ & $1.5 \pm 0.5$ \\
$0.7 \pm 0.3$ & $1.0 \pm 0.4$ \\
\hline
\end{tabular}

Abbreviations: $P D V=$ portal-drained viscera. Pigs at Day 1 during endotoxin infusion received L-Ala (Con) or L-Arg (Arg) intravenously and at Day 2 during recovery from endotoxin infusion received L-Ala or L-Arg intragastrically. Con group; $n=6$, Arg group, $n=8$. Data are mean $\pm S E M$ in $\mu$ mol $\bullet \mathrm{kg} \mathrm{bw}^{-1} \cdot \mathrm{min}^{-1}$. Statistics by Mann-Whitney $U:{ }^{*} P<0.05,{ }^{\star \star} P<0.01$; significant difference between the groups at Day 1 or 2 .

Table 4: Disposal and production of citrulline by the portal-drained viscera and kidneys.

\begin{tabular}{|c|c|c|c|c|c|c|c|}
\hline & & \multicolumn{6}{|c|}{ Day 1} \\
\hline & & \multicolumn{6}{|c|}{ FASTING ENDOTOXEMIA } \\
\hline & & \multicolumn{2}{|r|}{ Con } & \multicolumn{3}{|c|}{ Arg } & $P$ \\
\hline \multirow[t]{2}{*}{ PDV } & Disposal & -141 & \pm 42 & -89 & \pm & 46 & \\
\hline & Production & 496 & \pm 32 & 263 & \pm & 44 & * \\
\hline \multirow[t]{2}{*}{ Kidney } & Disposal & -891 & \pm 130 & -130 & \pm & 106 & ** \\
\hline & Production & 337 & \pm 146 & 37 & \pm & 76 & * \\
\hline
\end{tabular}

\begin{tabular}{rrr}
\hline \multicolumn{4}{c}{ Day 2} \\
\hline \multicolumn{3}{c}{ FED POST-ENDOTOXEMIA } \\
\hline Con & \multicolumn{1}{c}{ Arg } & $P$ \\
\hline$-217 \pm 152$ & $-61 \pm 69$ \\
$622 \pm 156$ & $272 \pm 90$ \\
$-715 \pm 131$ & $-480 \pm 97$ \\
$92 \pm 196$ & $5 \pm 106$ \\
\hline
\end{tabular}

Abbreviations: $P D V=$ portal-drained viscera. Pigs at Day 1 during endotoxin infusion received L-Ala (Con) or L-Arg (Arg) intravenously and at Day 2 during recovery from endotoxin infusion received L-Ala or L-Arg intragastrically. Con group; $n=6$, Arg group, $n=8$. Data are mean $\pm S E M$ in $n$ mol $\bullet \mathrm{kg} \mathrm{bw}^{-1} \cdot \mathrm{min}^{-1}$. Statistics by Mann-Whitney $U:{ }^{*} P<0.05,{ }^{\star \star} P<0.01$; significant difference between the groups at Day 1 or 2 .

Table 5: Cit $\rightarrow$ Arg flux representing arginine de novo synthesis in the whole body, portal-drained viscera and kidneys measured from $C_{1} D_{2}$-Cit to $C_{1} D_{2}$-Arg isotope conversion.

\begin{tabular}{|c|c|c|c|c|c|c|}
\hline & \multicolumn{3}{|c|}{ Day 1} & \multicolumn{3}{|c|}{ Day 2} \\
\hline & \multicolumn{3}{|c|}{ FASTING ENDOTOXEMIA } & \multicolumn{3}{|c|}{ FED POST-ENDOTOXEMIA } \\
\hline & Con & $\operatorname{Arg}$ & $P$ & Con & Arg & $P$ \\
\hline \multirow[t]{3}{*}{$\mathrm{Cit} \rightarrow \mathrm{Arg}$} & $384 \pm 57$ & $158 \pm 21$ & $*$ & $565 \pm 60$ & $393 \pm 51$ & $*$ \\
\hline & $80 \pm 36$ & $71 \pm 50$ & & n.d. & n.d. & \\
\hline & $211 \pm 39$ & $17 \pm 86$ & ** & $210 \pm 177$ & $131 \pm 111$ & \\
\hline
\end{tabular}

Abbreviations: $W B=$ whole body, $P D V=$ portal-drained viscera, $n . d .=$ not determined. Pigs at Day 1 during endotoxin infusion received L-Ala (Con) or L-Arg (Arg) intravenously and at Day 2 during recovery from endotoxin infusion received L-Ala or L-Arg intragastrically. Con group; $n=6$, Arg group, $n=8$. Data are mean

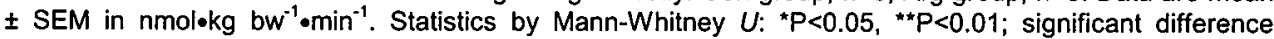
between the groups at Day 1 or 2 . 
Portal-drained viscera: Figure $2 B$ shows that arginine infusion during and after endotoxin infusion induced arginine influx into the portal-drained viscera, concomitant to increased arginine disposal and production (Table 3). The citrulline efflux from the portal-drained viscera decreased (Figure $2 B$ ) during arginine infusion owing to decreased citrulline production (Table 4). Arginine treatment in endotoxemic and postendotoxemic pigs induced ornithine influx and abolished glutamine influx into the portal-drained viscera (Figure 2B). In contrast, ammonia efflux from the portal-drained viscera was not affected by arginine administration. Arginine infusion during endotoxin infusion also did not affect the arginine de novo synthesis in the portal-drained viscera (Table 5), which proportioned to about $20 \%$ of the whole-body de novo arginine synthesis in the Con and about $40 \%$ in the Arg group. No reliable arginine de novo synthesis could be calculated across the portal-drained viscera during feeding because of interference of dietary-derived arginine production with $\mathrm{C}_{1} \mathrm{D}_{2}$-arginine portal-arterial enrichment differences. Elevated concentrations of arginine and ornithine and reduced concentrations of glutamine and citrulline in mucosal tissue of the jejunum (Table 6) were observed. Moreover, supplementation of arginine during and after endotoxin infusion led to a decrease in ammonia concentration in the jejunal biopsies of the endotoxin-infused pigs, nevertheless, did not exert any effect on measured polyamine concentrations (Table 6).
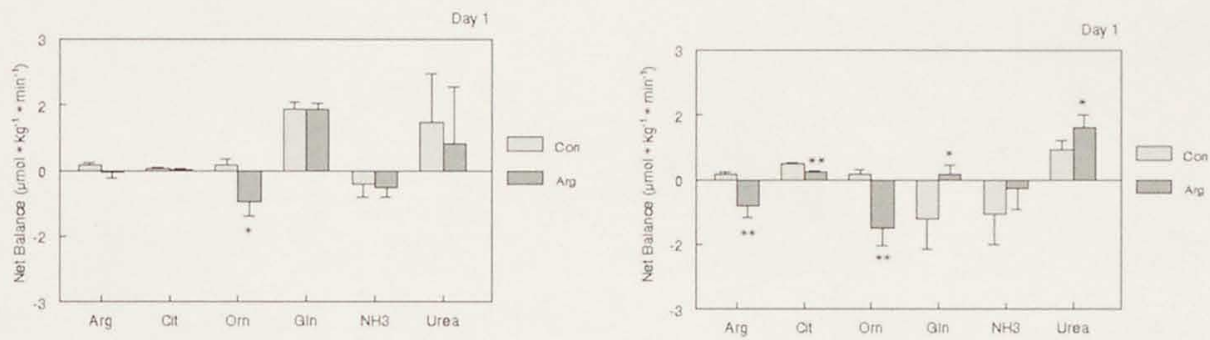

Day 2
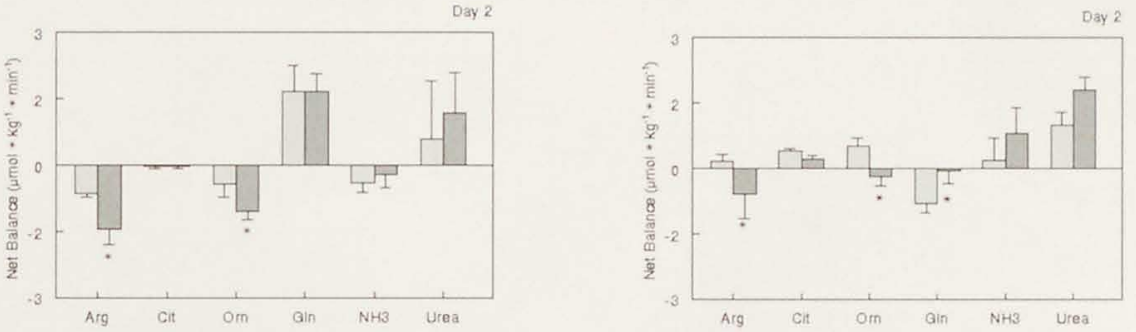

Figure 2 A, B. Urea cycle-related substrate net balances across (A): the hindquarter and (B): the portaldrained viscera in pigs at Day 1 during endotoxin infusion receiving L-Ala (Con) or L-Arg (Arg) intravenously and at Day 2 during recovery from endotoxin infusion receiving L-Ala or L-Arg intragastrically. Statistics by Mann-Whitney $U:{ }^{\star} P<0.05,{ }^{\star \star} P<0.01$; significant difference between the groups at Day 1 or 2 . 
Table 6: Tissue amino acid, ammonia and polyamine concentrations in jejunal mucosa biopsies.

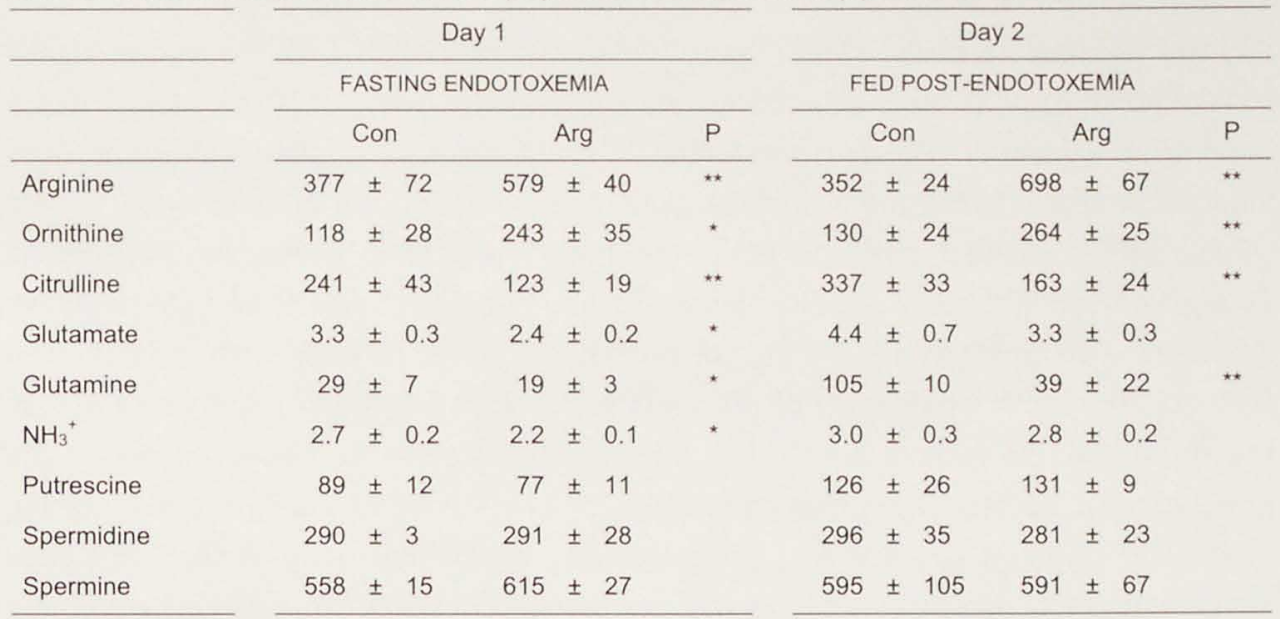

Abbreviations: $\mathrm{NH}_{3}{ }^{+}=$ammonia. Pigs at Day 1 during endotoxin infusion received L-Ala (Con) or L-Arg (Arg) intravenously and at Day 2 during recovery from endotoxin infusion received L-Ala or L-Arg intragastrically. Con group; $n=6$, Arg group, $n=8$. Data are mean \pm SEM in in $\mu \mathrm{mol} / \mathrm{kg}$ wet weight, except for glutamate and $\mathrm{NH}_{3}{ }^{+}$(in $\mathrm{mmol} / \mathrm{kg}$ wet weight). Statistics by Mann-Whitney $U:{ }^{*} \mathrm{P}<0.05,{ }^{\star \star} \mathrm{P}<0.01$; significant difference between the groups at Day 1 or 2 .
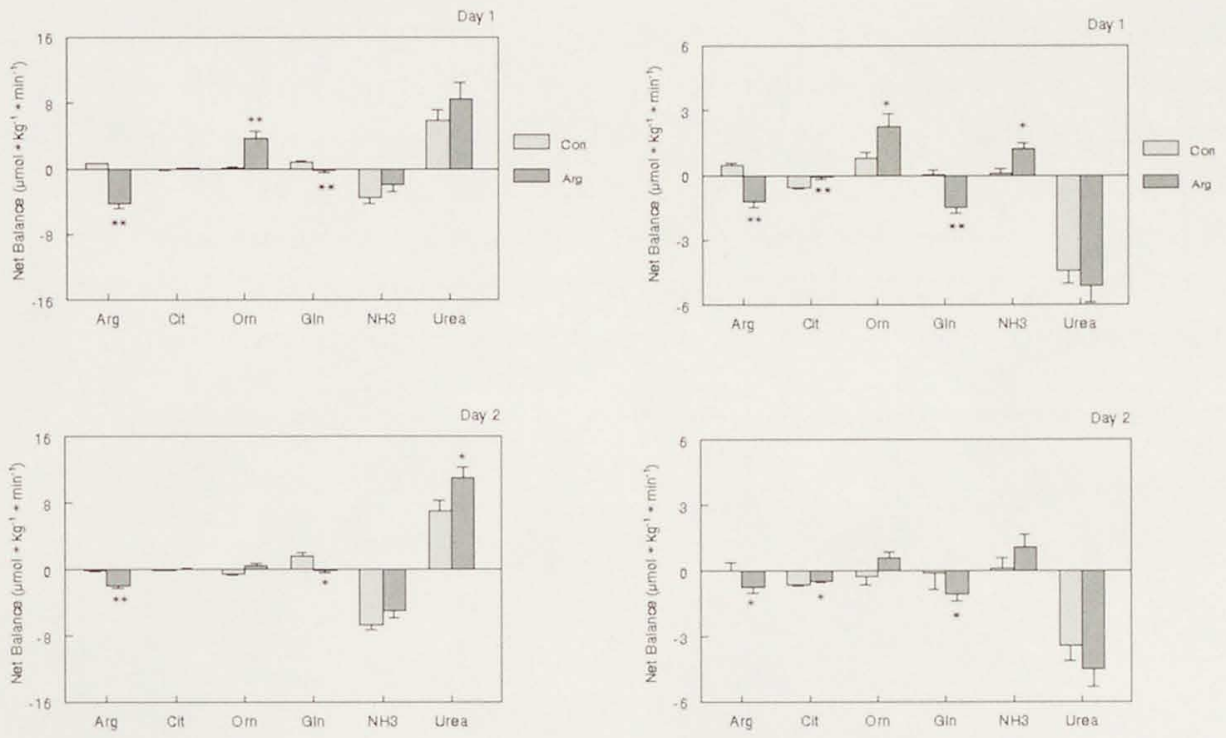

Figure $2 \mathrm{C}, \mathrm{D}$. Urea cycle-related substrate net balances across (C): the liver and (D): the kidneys in pigs at Day 1 during endotoxin infusion receiving L-Ala (Con) or L-Arg (Arg) intravenously and at Day 2 during recovery from endotoxin infusion receiving $L$-Ala or $L$-Arg intragastrically. Statistics by Mann-Whitney $U$ : ${ }^{*} \mathrm{P}<0.05,{ }^{* *} \mathrm{P}<0.01$; significant difference between the groups at Day 1 or 2 . 
Liver: The hepatic arginine influx increased 5 -fold when i.v. arginine treatment was given at Day 1 (Figure 2C) and increased 4-fold during intragastric arginine infusion the following day. At both days, this was accompanied with elevated arginine disposal and production rates by the liver (Table 3). Arginine infusion induced ornithine net efflux from the liver and glutamine influx into the liver during endotoxin treatment and during recovery thereof (Figure 2C). The liver urea efflux was higher in the arginine treated than in the control animals at both Days 1 and 2. No significant net balance of citrulline was detectable across the liver in any of the groups. The citrulline disposal by the liver (not shown) was too low to calculate reliable arginine de novo production from $\mathrm{C}_{3}$-citrulline to $\mathrm{C}_{3}$-arginine cycling.

Kidneys: The i.v. infusion of arginine during endotoxemia converted arginine efflux across the kidneys into influx, as well did the intragastric arginine infusion postendotoxemia (Figure 2D). This occurred in parallel with increased renal arginine disposal that exceeded increased arginine production (Table 3). When arginine was administered to endotoxemic and postendotoxemic pigs, the citrulline influx into the kidneys (Figure 2D) decreased. Also, the citrulline disposal rate by the kidneys (Table 4) was less in arginine treated than in control pigs. During endotoxin infusion, but not the following day, this was accompanied by a significant decrease in $\mathrm{C}_{3}$-citrulline to $\mathrm{C}_{3}$-arginine conversion (Table 5). The relative contribution of this renal arginine de novo synthesis to the wholebody de novo synthesis of arginine (Figure $1 B$ ) amounted to $50 \%$ in the postabsorptive state and $35 \%$ in the fed state independent on arginine supplementation. When endotoxin challenged pigs were given arginine infusion, a marked efflux of ornithine from the kidneys was observed (Figure 2D). Arginine also induced substantial renal glutamine influx both during and after endotoxemia. The ammonia efflux remained unaffected by arginine supplementation. ${ }^{13} \mathrm{C}$-Urea isotope measurements demonstrated that arginine infusion enhanced both the renal urea excretion and synthesis on Day 1 (Table 7).

Table 7: Excretion and synthesis of urea by the kidney.

\begin{tabular}{|c|c|c|c|c|}
\hline & & \multicolumn{3}{|c|}{ Day 1} \\
\hline & & \multicolumn{3}{|c|}{ FASTING ENDOTOXEMIA } \\
\hline & & Con & Arg & $P$ \\
\hline \multirow[t]{3}{*}{ Kidney } & Excretion & $5.4 \pm 2$ & $10.7 \pm 3.3$ & ** \\
\hline & Production & $0.9 \pm 1.2$ & $6.6 \pm 2.4$ & $*$ \\
\hline & NB & $-4.5 \pm 0.6$ & $-4.1 \pm 0.8$ & \\
\hline
\end{tabular}

\begin{tabular}{ccc}
\hline \multicolumn{4}{c}{ Day 2 } \\
\hline \multicolumn{3}{c}{ FED POST-ENDOTOXEMIA } \\
\hline Con & Arg & $P$ \\
\hline $9.6 \pm 2.3$ & $17.1 \pm 4.5$ & \\
$6.2 \pm 2.1$ & $10.6 \pm 3.8$ & \\
$-3.4 \pm 0.7$ & $-6.5 \pm 0.8$ & \\
\hline
\end{tabular}

Pigs at Day 1 during endotoxin infusion received L-Ala (Con) or L-Arg (Arg) intravenously and at Day 2 during recovery from endotoxin infusion received L-Ala or L-Arg intragastrically. Con group; $n=6, \operatorname{Arg}$ group, $n=8$. Data are mean $\pm S E M$ in $\mu$ mol $\bullet \mathrm{kg}^{-1} \cdot \mathrm{min}^{-1}$. NB: - represents net disappearance. Statistics by MannWhitney $U:{ }^{*} P<0.05,{ }^{\star *} P<0.01$; significant difference between the groups at Day 1 or 2 . 


\section{Discussion}

In pigs, like in humans, a need for arginine may develop under conditions of stress such as trauma and sepsis (29). As a model of sepsis with therapeutic intervention, arginine was administered via the intravenous route to endotoxemic pigs and via the intragastric route to the same pigs recovering from the endotoxemia. Recently, we observed that, under endotoxemic and post-endotoxemic conditions, arginine supplementation exerted a stimulatory effect on the NO formation by different organs (in press, Crit Care Med, Bruins et al., 2001). In addition to stimulation of the NO synthase pathway, exogenous arginine supply may result in adaptive changes in arginine synthesis and degradation. The purpose of this study was to obtain insight into the effects of arginine supplementation under disease conditions on arginine and related intermediate metabolism and regulation of arginine homeostasis. The use of a multi-catheterized pig model under steady state conditions not only enabled in vivo flux measurements at whole-body level but also across the organs. Moreover, the use of stable isotopes extended the model with data on arginine and citrulline disposal, production and arginine de novo synthesis.

The infusion rate of arginine was chosen in the range of previously used arginine infusions for therapeutic intervention under disease conditions. When intravenously administered under endotoxemic conditions, arginine increased the whole-body arginine appearance rate 3-fold, whereas upon enteral infusion in the post-endotoxemic condition, arginine was partially metabolized by the portal-drained viscera and liver so that a 2-fold increase in whole-body arginine appearance rate was realized. The increase in arginine metabolism in the liver and kidneys seems to correlate well with increased efflux of ornithine and urea from these organs, two organs with high arginase content $(11,15)$. Accordingly, urea isotope measurements showed that most of the infused arginine was metabolized to urea. Arginine infusion also induced higher arginine disposal in the muscle and intestine probably reflecting increased degradation by arginase present in these tissues (34). Arginine supply decreased the whole body endogenous arginine appearance rate, which could partially be attributed to lowered arginine de novo synthesis. Protein turnover measurements in the same pig model (accepted, Bruins et al, Am J Clin Nutr, 2001) established that the remainder of the decrease in endogenous arginine appearance rate arises from a reduction in the rate of arginine leaving or entering plasma from protein synthesis/breakdown during and after endotoxemia. The relative contribution of the de novo synthesis of arginine measured from citrulline to arginine recycling to total endogenous flux amounted to $16-20 \%$ in fasted and fed conditions, respectively. These values are comparable to estimations of Castillo et al. (6) in healthy adults. In the pig under fasting endotoxemic conditions, the arginine de novo synthesis by the portal-drained viscera were $20-25 \%$ and the kidneys $50-35 \%$ 
accountable for the whole body arginine de novo synthesis. This finding implies that the intestine, in spite of relatively high arginase activity (34), is capable of arginine biosynthesis from citrulline. The liver and hindquarter citrulline net balances were too low to accurately measure citrulline disposal rates and conversion rates into arginine. The reason for this might be that in the hindquarter muscle, like in the liver, citrulline is tightly channeled to arginine by argininosuccinate synthase (ASS) and argininosuccinate lyase (ASL) (31). The citrulline output from the liver and hindquarter during and after endotoxemia in fasted and fed state, therefore, did not quantitatively contribute to the whole-body citrulline appearance. During arginine infusion under endotoxemic conditions, increased arginine concentration occurred with decreased glutamine and citrulline concentrations. Similar as to our findings, Beaumier at al. (2) showed that citrulline plasma levels in healthy humans fed an arginine-enriched diet were reduced. Hence, arginine appears the principal source of plasma ornithine, but plasma ornithine not of citrulline. Decreased glutamine levels are consistent with findings of Boza et al. (3) who showed that under unstressed conditions, addition of arginine to the diet led to a decrease in plasma glutamine concentration due to impaired de novo synthesis of glutamine.

In this model of endotoxemia, both intravenous and intragastric arginine supplementation involved net arginine utilization by the organs. Isotope measurements pointed out that this was due to increased arginine disposal exceeding a concomitant increase in arginine production. The increase in organ arginine disposal and urea release fits with the high $\mathrm{K}_{\mathrm{m}}$ of arginase and the ubiquitous distribution of this enzyme (16).

\section{Liver}

in agreement with in vitro data that arginine transport into liver cells is up-regulated by endotoxin (22), our in vivo data show enhanced arginine metabolism by the liver. Urea and ornithine efflux from the liver increased in parallel with glutamine influx, which may reflect elevated liver arginase activity during arginine supplementation. It was indeed shown that dietary arginine supplementation in perfused rat liver leads to induced urea production (8). The observed conversion of net release of glutamine into net uptake during arginine supplementation seems consistent with the opposite flux in rats fed an arginine-deficient diet (17). Reduced liver glutamine synthesis may account for this since activity of glutamine synthetase was found reduced in rats fed an arginine-enriched diet (3). Excess urea cycle intermediates (mainly, arginine) prevents escape of ammonia which served glutamine synthesis by high affinity glutamine synthetase in perivenous hepatocytes (18). Increased ornithine was shown to stimulate urea cycle activity and subsequent ammonia consumption. Enhanced hepatic glutamine consumption during 
arginine supplementation, therefore, can as well result from increased glutaminase activity involved in accelerated urea cycling (19).

\section{Kidneys}

Although the kidney normally possesses the capacity to synthesize arginine from citrulline (11), high arginine levels in endotoxemic and postendotoxemic pigs appeared capable of converting the renal arginine efflux into influx since arginine was increasingly metabolized. The kidneys displayed extra ornithine efflux and metabolic urea formation suggesting this arginine metabolism to represent arginase II-mediated arginine to ornithine conversion (21). Moreover, arginine addition may have stimulated the conversion of arginine into citrulline and NO (in press, Bruins et al., Crit Care Med, 2001). In the arginine-treated pigs, ammonia levels were relatively low. The observed increased glutamine net uptake by the kidneys therefore rather seems to result from decreased renal glutamine synthetase activity since increased glutamine degradation into urinary ammonia by increased glutaminase activity (7) would be expected as an aternative pathway to dispose excess ammonia from the body.

\section{Portal-drained viscera}

Arginine administration to endotoxemic pigs resulted in substantially increased arginine metabolism by the portal-drained viscera. Besides the arginine concentration, the ornithine concentration of mucosal tissue was elevated which may reflect increased arginine degradation by arginase II which is present in the small intestine (15). Despite the high tissue concentrations of ornithine, the concentration of the polyamines putrescine, spermine and spermidine, and, therefore, the ornithine decarboxylase (ODC) activity appeared not to be stimulated. Arginine infusion in endotoxemic and postendotoxemic pigs completely blunted glutamine influx into the portal-drained viscera. The enhanced urea cycle activity in the gut probably involves reduced intracellular glutamine availability. Glutamine net uptake and citrulline net release decreased simultaneously, implying impaired glutamine to citrulline flux in the gut in presence of high arginine levels. Also, free glutamine and citrulline levels in the jejunal mucosa were lower during arginine treatment. These findings are comparable with decreased glutamine and citrulline fluxes and concentrations in the jejenum of bowel-resected rats given parenteral arginine (32). Observed decreased citrulline efflux during arginine administration may well originate from decreased intracellular availability of glutamine since ammonia-derived carbamoyl phosphate acts as a rate-limiting substrate in the citrulline formation (31). The increased amounts of ornithine in the gut seem neither to be directed towards citrulline, nor towards polyamines, conversion via ornithine aminotransferase (OAT) into proline or $\alpha$ ketoglutarate (1) providing an alternative route. Accelerated ornithine oxidation would 
involve increased ornithine and glutamate disposal. Measurements of glutamine turnover and non-hepatic glutaminase activity would provide more insight in this process.

\section{Hindquarter}

Although arginine administration induced arginine uptake across the hindquarter muscle, citrulline and urea net balances remained unaffected. This implies that arginase activity in muscle is very low. The ornithine flux into the hindquarter increased to a remarkable extent by arginine. Possibly, elevated ornithine concentrations in the muscle are processed through increased conversion into proline $(12,23)$ or oxidation via the entry of glutamate in the TCA cycle. Arginine supplementation in this endotoxemia model did not exert any effect on the glutamine flux across the hindquarter which, nevertheless, may not exclude concurrent changes in glutamine disposal and production rate. Arginine supplementation in rats, however, showed not to affect glutamine synthetase and glutamine activity in muscle tissue (3).

Except for the muscle, arginine did not increase protein turnover in the different organs (unpublished results). Considering that the visceral and renal arginine de novo synthesis also did not increase by arginine, we could not explain the increased organ arginine production that was measured from ${ }^{15} \mathrm{~N}_{2}$-arginine. Because of the large arginine pool in the arginine-treated animals, steady state conditions for ${ }^{15} \mathrm{~N}_{2}$-arginine may not entirely have been reached which could have contributed to an overestimation of the organ ${ }^{15} \mathrm{~N}_{2}$ arginine net balance and subsequent disposal and production rates.

The reduced citrulline synthesis by the portal-drained viscera appears to determine the inhibited citrulline to arginine conversion rate in the kidney. The lowered citrulline flux between portal-drained viscera and kidney observed in endotoxemic and postendotoxemic pigs treated with arginine would explain the observed decrease in whole-body citrulline turnover rate. The inhibited renal arginine de novo synthesis primarily accounted for the decreased arginine de novo synthesis observed at wholebody level. Arginine was found to stimulate hepatic glutamine influx and renal glutamine influx, whereas it inhibited visceral glutamine influx. Elevated glutamine utilization by both the kidney and the liver could have contributed to reduced plasma glutamine levels.

This model of endotoxemia and recovery with arginine supplementation shows that multiple organs are involved in the in vivo organization of the glutamine-citrulline-arginine metabolic axis. The present findings imply that arginine, citrulline and glutamine metabolism are strongly related. Both liver and kidneys appear a main source of glutamine consumption, for the purpose of urea and ammonia genesis, respectively. Provided that arginase is present in virtually all organs, increased arginine supply resulted in increased organ arginine conversion into ornithine and urea and increased 
tissue ornithine levels were demonstrated in the mucosal tissue of the jejunum. Although OTC in the gut is relatively abundant, the absence of increased citrulline synthesis in spite of elevated intracellular ornithine leveis in the jejunum could arise from limited local glutamine and ammonia availability. It might be speculated that decreased citrulline release from the portal-drained viscera into the circulation becomes restrictive for renal arginine synthesis, thereby constituting a negative feed-back regulation mechanism of de novo arginine synthesis.

In conclusion, stable isotope measurements showed that although exogenous intravenous or intragastric arginine under endotoxemic conditions promoted both the ornithine and urea synthesis during prolonged endotoxemic conditions, it does not serve as an effective precursor of citrulline. Moreover, arginine administration inhibited the citrulline appearance rate and citrulline to arginine conversion at whole-body level. The decreased plasma availability of glutamine, hence, substrate for citrulline, may be the primary cause for the impaired citrulline production by the portal-drained viscera and ensuing reduced citrulline disposal by the kidneys. This would be according to the proposal that the ornithine to citrulline conversion rate in plasma and the portal-drained viscera is determined by glutamine availability (6). In summary, the arginine homeostasis seems achieved by both elevated arginine degradation and decreased endogenous arginine production.

\section{Acknowledgments}

The authors wish to thank Mr. H.M.H. van Eijk and Mr. J.L.J.M. Scheijen for analytical amino acid measurements and Mrs. G.A.M. ten Have for assistance with operational procedures. This research was supported by a grant from the Netherlands Organization for Scientific Research (902-23-098).

\section{References}

1. Alonso, E., and V. Rubio. Participation of ornithine aminotransferase in the synthesis and catabolism of ornithine in mice. Biochem $J$ 259: 131-138, 1989.

2. Beaumier, L., L. Castillo, A. M. Ajami, and V. R. Young. Urea cycle intermediate kinetics and nitrate excretion at normal and "therapeutic" intakes of arginine in humans. Am Physiol Soc 269: E884-E896, 1995.

3. Boza, J. J., D. Moennoz, A. R. Jarret, J. Vuichoud, C. Garcia-Rodenas, P. A. Finot, and O. Ballevre. Neither glutamine nor arginine supplementation of diets increase glutamine body stores in healthy growing rats. Clin Nutr 19: 319-325, 2000.
4. Bruins, M. J., P. B. Soeters, and N. E. Deutz. Endotoxemia affects organ protein metabolism differently during prolonged feeding in pigs. $J$ Nutr 130: 3003-13, 2000.

5. Castillo, L., T. E. Chapman, Y. M. Yu, A. Ajami, J. F. Burke, and V. R. Young. Dietary uptake by the splanchnic region in adult humans. Am J Physiol 265: E532-E539, 1993.

6. Castillo, L., M. Sanchez, J. Vogt, T. E. Chapman, T. C. DeRojas-Walker, S. R. Tannenbaum, A. M. Ajami, and V. R. Young. Plasma arginine, citrulline, and ornithine kinetics in adults, with observations on nitric oxide synthesis. Am J Physiol 268: E360E367, 1995. 
7. Curthoys, N. P., and M. Watford. Regulation of glutaminase activity and glutamine metabolism. Annu Rev Nutr 15: 133-59, 1995.

8. De Bandt, J. P., L. Cynober, S. K. Lim, C. Coudray-Lucas, R. Poupon, and J. Giboudeau. Metabolism of ornithine, alpha-ketoglutarate and arginine in isolated perfused rat liver. $\mathrm{Br} J$ Nutr 73: 227-39., 1995.

9. Dejong, C. H., N. E. Deutz, and P. B. Soeters. Renal ammonia and glutamine metabolism during liver insufficiency- induced hyperammonemia in the rat. $J$ Clin Invest 92: 2834-40., 1993.

10. Desmukh, D. R., V. S. Ghole, B. Marescau, and P. P. De Deyn. Effect of endotoxemia on plasma and tissue levels of nitric oxide metabolites and guanidino compounds. Arch Physiol Biochem 105: 32-37, 1997.

11. Dhanakoti, S. N., M. E. Brosnan, G. R. Herzberg, and J. T. Brosnan. Cellular and subcellular localization of enzymes of arginine metabolism in rat kidney. Biochem J 282: 369375, 1992.

12. Edmonds, M. S., K. R. Lowry, and D. H. Baker. Urea cycle metabolism: effects of supplemental ornithine or citrulline on performance, tissue amino acid concentrations and enzymatic activity in young pigs fed arginine-deficient diets. J Anim Sci 65: 706-16, 1987.

13. Evoy, D., M. D. Lieberman, T. J. Fahey, 3rd, and J. M. Daly. Immunonutrition: the role of arginine. Nutrition 14: 611-7, 1998.

14. Fink, M. P., and S. O. Heard. Laboratory models of sepsis and septic shock. J Surg Res 49: 186-196, 1990.

15. Gotoh, T., M. Araki, and M. Mori. Chromosomal localisation of the human arginase II gene and tissue distribution of its mRNA. Biochem Biophys Res Commun 233: 487-491, 1997.

16. Greengard, O., M. K. Sahib, and W. E. Knox. Developmental formation and distribution of arginase in rat tissues. Arch Biochem Biophys 137: 477-82, 1998.

17. Hartman, W. J., and R. L. Prior. Dietary arginine deficiency alters flux of glutamine and urea cycle intermediates across the portaldrained viscera and liver of rats. $J$ Nutr 122: 1472-82., 1992.

18. Haussinger, D. Hepatocyte heterogeneity in glutamine and ammonia metabolism and the role of an intercellular glutamine cycle during ureogenesis in perfused rat liver. Eur $J$ Biochem 133: 269-75., 1983.
19. Haussinger, D., H. Sies, and W. Gerok. Functional hepatocyte heterogeneity in ammonia metabolism. The intercellular glutamine cycle. J Hepatol 1: 3-14, 1985.

20. Lortie, M. J., S. Ishizuka, D. Schwartz, and R. C. Blantz. Bioactive products of arginine in sepsis: tissue and plasma composition after LPS and iNOS blockade. Am J Physiol Cell Physiol 278: C1191-9., 2000.

21. Mori, M., T. Gotoh, A. Nagasaki, M. Takiguchi, and $T$. Sonoki. Regulation of the urea cycle enzyme genes in nitric oxide synthesis. $J$ Inher Metab Dis 21: 59-71, 1998.

22. Pacitti, A. J., E. M. Copeland, and W. W. Souba. Stimulation of hepatocyte system $y+-$ mediated L-arginine transport by an inflammatory agent. Surgery 112: 403-411, 1992.

23. Pardridge, W. M., L. Duducgian-Vartavarian, D. Casanello-Ertl, M. R. Jones, and J. D. Kopple. Arginine metabolism and urea synthesis in cultured rat skeletal muscle cells. Am J Physiol 242: E87-92, 1982.

24. Roland, C. R., Y. Nakafusa, and M. W. Flye. Gadolinium chloride inhibits lipopolysaccharide-induced mortality and in vivo prostaglandin E2 release $B y$ splenic macrophages. J Gastrointest Surg 3: 301-7., 1999.

25. Salimuddin, A. Nagasaki, T. Gotoh, H. Isobe, and M. Mori. Regulation of the genes for arginase isoforms and related enzymes in mouse macrophages by lipopolysaccharide. Am J Physiol 277: E110-7, 1999.

26. Ten Have, G. A. M., M. C. F. Bost, J. C. A. W. Suyk-Wierts, A. E. J. M. van den Bogaart, and N. E. P. Deutz. Simultaneous measurement of metabolic flux in portally-drained viscera, liver, spleen, kidney and hindquarter in the conscious pig. Lab Anim 30: 347-358, 1996.

27. Van Eijk, H. M. H., D. R. Rooyakkers, and N. E. P. Deutz. Automated determination of polyamines by high-performance liquid chromatography with simple sample preparation. $J$ Chromatogr 730: 115-120, 1996.

28. Van Eijk, H. M. H., D. R. Rooyakkers, and N. E. P. Deutz. Determination of amino acid isotope enrichment using liquid chromatography-mass spectrometry. Anal Biochem 271: 8-17, 1999.

29. Visek, W. J. Arginine and disease states. $J$ Nutr 115: 532-41, 1985. 
30. Wang, W. W., C. P. Jenkinson, J. M. Griscavage, R. M. Kern, N. S. Arabolos, R. E. Byrns, S. D. Cederbaum, and L. J. Ignarro. Coinduction of arginase and nitric oxide synthase in murine macrophages activated by lipopolysaccharide. Biochem Biophys Res Commun 210: 1009-16., 1995.

31. Watford, M. The urea cycle: a twocompartment system. Essays Biochem 26: 49$58,1991$.
32. Welters, C. F., C. H. Dejong, N. E. Deutz, and E. Heineman. Effects of parenteral arginine supplementation on the intestinal adaptive response after massive small bowel resection in the Rat. J Surg Res 85: 259-66, 1999.

33. Wolfe, R. R. Radioactive and stable isotope tracers in biomedicine. Principles and practice of kinetic analysis. New York: Wiley-Liss, 1992.

34. Wu, G. Synthesis of citrulline and arginine from proline in enterocytes of postnatal pigs. Am J Physiol 272: G1382-G1390, 1997. 


\author{
Maaike J Bruins \\ Yvette C Luiking \\ Peter B Soeters \\ Louis MA Akkermans* \\ Nicolaas EP Deutz
}

Department of Surgery, Maastricht University, The Netherlands

-Department of Surgery, University of Utrecht, The Netherlands 


\section{Abstract}

Motility disorders of the gastrointestinal tract are a common complication of sepsis. It has been suggested that gram-negative endotoxin plays a role in the pathogenesis of the accompanying diarrhea frequently observed.

To determine the effects of hyperdynamic endotoxemia on the motility of the small intestine, pigs were infused with $E$. coli lipopolysaccharide for a 24-h period. During this fasting period jejunal motility was measured by using ambulatory manometry. One and 4 days after cessation of endotoxin, pigs were enterally fed and, again, motility was recorded.

Hyperdynamic endotoxemia was achieved in this model. Manometric pressure recordings revealed that endotoxin infusion accelerated the migrating motor complex (MMC) migration along the jejunum. Also, a simultaneous increase in MMC cycling frequency was observed in endotoxin-treated group. Elevated MMC migration velocity and cycling frequency were maintained the following day postendotoxin during feeding and returned to basal values 4 days postendotoxin. Therefore, a small dose continuously infused endotoxin significantly provokes jejunal motility disturbances that may contribute to diarrhea.

\section{Introduction}

Disturbed gastrointestinal motility is a frequently observed complication in septic patients. In septic patients, the complex interaction between anesthesia, surgical manipulation, inflammatory agents, nutritional status and hormone levels determine the picture of altered motility (for review see: (24)). Retarded passage in the large intestine leads to constipation and in the advanced stages to ileus whereas the main symptom in accelerated passage of the large intestine is diarrhea (24). Challenge with bacterial endotoxins is a common way to imitate septic shock or sepsis (6). Intravenous Escherichia coli endotoxin administration was found to affect small intestinal motility in rats (4), piglets (10) and dogs (18). Several lines of evidence suggest that endotoxininduced mediators like neuropeptides, cytokines and nitric oxide (NO) are involved in the disturbances of gastrointestinal motility observed in endotoxemia $(5,7,12,17,26)$. Besides these mediators, the rate of intestinal blood supply plays an important factor in the gut functioning and motility (8).

In the small bowel, the fasting gastrointestinal motility pattern, the migrating motor complex (MMC) has been attributed a housekeeper function, and its maintenance considered essential in the prevention of gut-derived infections (16). The effect of hyperdynamic entotoxemia with preserved visceral blood flow on small intestinal motor 
activity has not been well characterized. Therefore, the aim of this study was to investigate the effects of prolonged hyperdynamic endotoxemia on the MMC pattern of the small intestine. In contrast to previous endotoxemia models in which single sub-lethal doses of endotoxin were administrated, in this clinical model of sepsis continuous small doses of endotoxin were infused accompanied with fluid resuscitation. This experimental set-up has more resemblance with the hyperdynamic septic state (6) that is encountered in septic patients receiving modern intensive care. Moreover, as enteral nutrition is thought to play a role in the maintenance of gut function (11), postendotoxemic effects on motility were assessed during nutritional intervention.

\section{Materials and methods}

\section{Animals}

Twelve female crossbred pigs (Yorkshire $x$ Dutch Landrace: $20-22 \mathrm{~kg}$ body weight (bw)) were fed $1 \mathrm{~kg}$ of regular pig feed (Landbouwbelang, Roermond, the Netherlands; $16 \%$ crude protein) daily in the morning, supporting a growth rate of approximately $300 \mathrm{~g}$ per day. Before surgery, pigs were randomly assigned to the endotoxin treatment group $(n=6)$ or the control group $(n=6)$. The Animal Ethics Committee of the Maastricht University approved the study.

\section{Surgical procedure}

The night before surgery, the pigs were withheld from food. One hour after premedication with $10 \mathrm{mg} / \mathrm{kg}$ bw intramuscular azoperone (Stresnil, Janssen pharmaceutica, Beersse, Belgium), anesthesia was induced by a mixture of $\mathrm{N}_{2} \mathrm{O} / \mathrm{O}_{2}(1: 2)$ and halothane $(0.8 \%)$. After intubation, the pigs were intravenously administrated $6.25 \mathrm{mg} / \mathrm{kg}$ bw lincomycin. $2 \mathrm{HCl}$ (Lincomycin, A.U.V., Cuyk, the Netherlands) as bactericidal prophylaxis and $12.5 \mathrm{mg} / \mathrm{kg}$ bw spectinomycin. $\mathrm{HCl}$ lyophil (Spectinomycin, A.U.V.) as bacteriostatic prophylaxis. To avoid coagulation and as postoperative analgesia, $50 \mathrm{mg} / \mathrm{kg}$ bw flunixine (Finadyne, Schering-Ploegh, Brussel, Belgium) was given. During surgery, the anesthesia was maintained by the $\mathrm{N}_{2} \mathrm{O} / \mathrm{O}_{2}$-halothane mixture and I.V. given Lactetrol (per ml: $5.76 \mathrm{mg} \mathrm{NaCl}, 0.37 \mathrm{mg} \mathrm{KCl}, 0.37 \mathrm{mg} \mathrm{CaCl}, 0.2 \mathrm{mg} \mathrm{MgCl}_{2}, 5 \mathrm{mg}$ sodium lactate, methyl parahydroxy bezoicacid, Janssen pharmaceutica). The surgical procedure has been described in detail (23). Catheters were cannulated in (A1) the abdominal aorta with the end positioned just above the bifurcation, in $(P)$ the portal vein for blood sampling, in (V2) the inferior caval vein with the end positioned just above the right renal vein for endotoxin infusion and in (S) the splenic vein that was used for infusion of paraaminohippuric acid (PAH). A gastric tube (inner diameter $1.6 \mathrm{~mm}$, outer diameter 4.8 $\mathrm{mm}$, Tygon, Westvaco, Cleveland, $\mathrm{OH}, \mathrm{USA}$ ) was inserted for enteral feeding. The 
catheters were tunneled through the abdominal wall and skin. In addition, a Bishop-Koop stoma was constructed. Briefly, $20 \mathrm{~cm}$ distally of the Ligament of Treitz (the transition from duodenum to jejunum), the jejunum was transected and the anatomical continuity of the remaining bowel reestablished by a single layer running suture (PDS 4-0 synthetic polydioxanon; Ethicon, Norderstedt, Germany) creating an end-to-side anastomosis. The transsected distal part of the jejunum was sutured to the skin, creating a single barrel stoma. At experiment days, the manometry catheter was inserted via the stoma into the lumen of the small bowel.

During recovery of the pigs, the stoma was closed by a filled balloon catheter (Size Ch.16, 3-5 ml; Rüsch-Gold, Kernen, Germany) to avoid leakage of gastrointestinal juices from the jejunum. Pigs wore a canvas harness to protect the catheters and the stoma and to allow easy handling. On the first two postoperative days, pigs received twice-daily $6.25 \mathrm{mg} / \mathrm{kg}$ bw lincomycin and $12.5 \mathrm{mg} / \mathrm{kg}$ bw spectinomycin intravenously. Pigs were repeatedly placed in a movable cage to get accustomed to the situation during which the experiment would be carried out.

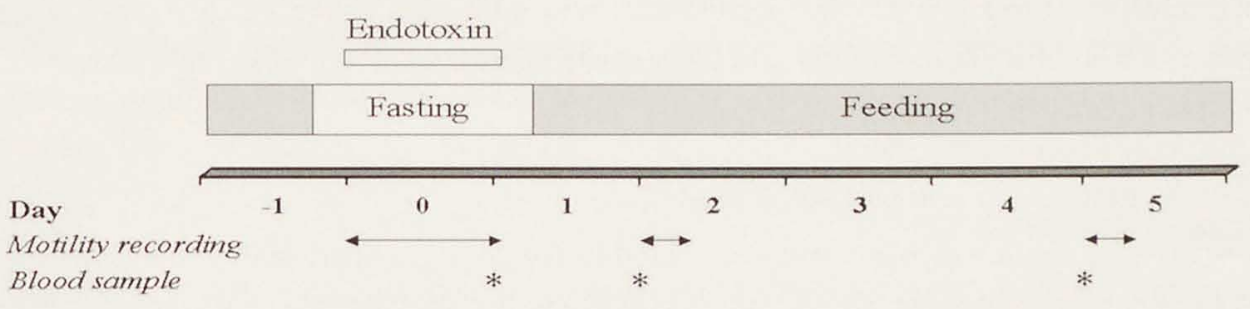

Figure 1. Schematic drawing of the experimental protocol. For blood flow measurements at Day 1, 2 and 5, blood was sampled in triplicate at arterial, portal and venous sites $60 \mathrm{~min}$ after start of para-aminohippuric acid infusion.

\section{Experimental protocol}

From the tenth postoperative day onwards, a high-protein liquid diet (Nutrison Steriflo Protein-Plus, Nutricia, Zoetermeer, the Netherlands) was enterally infused into the gastric tube via a swivel system connected to a pump. To get the pigs accustomed to the diet, enteral nutrition was infused at a rate of $4 \mathrm{~mL} \cdot \mathrm{kg} \mathrm{bw}^{-1} \cdot \mathrm{h}^{-1}\left(0.3 \mathrm{~g} \cdot \mathrm{kg} \mathrm{bw}{ }^{-1} \cdot \mathrm{h}^{-1}\right.$ of protein) for the first three days. On the tenth postoperative day, after an 8 -h fasting period, experiments were started. The design of the protocol is delineated in Figure 1. At $0 \mathrm{~h}$, an intravenous infusion of endotoxin (lipopolysaccharide from Escherichia coli, serotype 055:B5; Sigma Chemical Co., St. Louis, MO, USA) dissolved in saline was started at a rate of $3 \mu \mathrm{g} \cdot \mathrm{kg} \mathrm{bw} \mathrm{w}^{-1} \cdot \mathrm{h}^{-1}$. Endotoxin was infused for $24-\mathrm{h}$. Control pigs received saline at an equal infusion rate. All pigs were administrated extra saline infusion to secure replenishment of intravascular volume losses and to maintain visceral flow; 30 $\mathrm{ml} \cdot \mathrm{kg} \mathrm{bw}^{-1} \cdot \mathrm{h}^{-1}$ the first 8 hour and $20 \mathrm{ml} \cdot \mathrm{kg} \mathrm{bw}^{-1} \cdot \mathrm{h}^{-1}$ the following 16 hour. At $0 \mathrm{~h}$, the 
manometry catheter was inserted through the jejunal stoma into small intestine and 24-h motility recordings of the endotoxemic period were collected (Day 0). Eight hours after cessation of the endotoxin infusion, enteral nutrition was restarted at the infusion rate conducted before $\left(4 \mathrm{~mL} \cdot \mathrm{kg} \mathrm{bw}^{-1} \cdot \mathrm{h}^{-1}\right)$. One and 4 days after cessation of the endotoxin infusion, (Day 2 and 5, respectively), 8-h motility readings from the postprandial postendotoxemic pigs were obtained. At Day 1, Day 2 and Day 5, body temperature, mean arterial blood pressure and heart rate were measured. Also at these days, blood samples were collected to measure visceral blood flow (Figure 1).

\section{Infusion protocol}

At Day 1, Day 2 and Day 5, infusion protocols were performed to measure visceral blood flow. One hour before the start of the infusions, a primed constant infusion of paraaminohippuric acid (PAH, $25 \mathrm{mmol} / \mathrm{L}, \mathrm{A}$ 1422, Sigma Chemicals Co., St. Louis, MO, USA) was given. The PAH infusion was started at a rate of about $40 \mathrm{~mL} / \mathrm{h}$ for $1 \mathrm{~h}$ per catheter through the $S$ catheter after an initial bolus of $5 \mathrm{~mL}$ (23). Steady state condition for $\mathrm{PAH}$ was assessed in a pilot experiment in which blood samples were collected at 10min intervals. Steady state was achieved 60 min after start of the infusion (23). Blood samples from the arterial, portal and venous site were obtained in triplicate 60 min after the start of PAH infusion.

\section{Sample processing and biochemical analysis}

Directly after sampling, blood was distributed in pre-chilled, heparinized tubes (Sarstedt, Nümbrecht, Germany) on ice. For analysis of $\mathrm{PAH}, 300 \mu \mathrm{L}$ of whole blood was added to $600 \mu \mathrm{L}$ of $0.7 \mathrm{mmol} / \mathrm{L}$ TCA solution and thoroughly mixed. Supernatant was collected after centrifuging and stored at $-80^{\circ} \mathrm{C}$ until use. The $\mathrm{PAH}$ concentration in the supernatant was detected spectrophotometrically on an automated analysis system (Cobas Mira-S, Hoffmann-La Roche, Basel, Switzerland) as has been described before (23) by standard enzymatic methods, using commercially available kits.

\section{Calculations}

The portal-drained viscera are defined as the total of all portal-drained organs which, except for spleen, stomach and pancreas, largely represent the intestines. By sampling arterially and portally, the blood flow rate across portal-drained viscera can be calculated using the following formula based on the principle of indicator dilution methods in which I represents the rate of the $\mathrm{PAH}$ infused in the splenic vein.

$$
\text { Flow }{ }_{B L O O D}=1 /\left([\mathrm{PAH}]_{\mathrm{P}}-[\mathrm{PAH}]_{\mathrm{A}}\right)
$$

$[\mathrm{PAH}]_{\mathrm{P}}$ and $[\mathrm{PAH}]_{\mathrm{A}}$ represent the concentration of $\mathrm{PAH}$ in the portal and arterial blood, respectively. The dilution of $[\mathrm{PAH}]_{V}$ infused via the splenic vein entering the portal vein, depends on the blood flow rate through the portal-drained viscera. 


\section{Intestinal motility measurements}

During experiments, motility was studied by ambulatory manometry, using a 4 channel catheter with 4 solid-state pressure transducers (CTO-6, Dunvegan, Isle of Skye, Scotland) spaced 5-cm apart. The transducers were calibrated before the investigations were started. Immediately following start of the infusions, the manometry catheter was inserted into the bowel lumen of the jejunum via the Bishop-Koop jejunostomy, with the pressure transducers located at $10,15,20$ and $25 \mathrm{~cm}$ distal to the end-to-side anastomosis. Pressure signals were sampled at a frequency of $8 \mathrm{~Hz}$ and stored in a data logger (UPS-2020, MMS, Enschede, the Netherlands). A personal computer served as on-line monitoring and retrieval system. Recordings were performed for a period of 24-h (fasting) or 8-h (postprandial).

Table 1. Motility pattern in pigs.

\begin{tabular}{|c|c|c|c|c|c|c|c|}
\hline & & \multirow{2}{*}{\multicolumn{2}{|c|}{$\begin{array}{c}\text { FASTING } \\
\text { Day } 0\end{array}$}} & \multicolumn{4}{|c|}{ FED } \\
\hline & & & & \multicolumn{2}{|c|}{ Day 2} & \multicolumn{2}{|c|}{ Day 5} \\
\hline & & Control & ET & Control & ET & Control & ET \\
\hline \multirow[t]{3}{*}{ MMC } & Duration (min) & $59 \pm 8$ & $34 \pm 1$ & $54 \pm 5$ & $37 \pm 4$ & $53 \pm 2$ & $46 \pm 6$ \\
\hline & Frequency $\left(h^{-1}\right)$ & $1.1 \pm 0.2$ & $1.7 \pm 0$ & * $\quad 1.2 \pm 0.1$ & $1.5 \pm 0.1$ & - $\quad 1.1 \pm 0.1$ & $1.3 \pm 0.1$ \\
\hline & Migration $(\mathrm{mm} / \mathrm{min})$ & $18 \pm 1$ & $32 \pm 3$ & $* \quad 15 \pm 3$ & $34 \pm 3$ & - $16 \pm 2$ & $8 \pm 5$ \\
\hline \multirow[t]{2}{*}{ Contraction } & Frequency $\left(\min ^{-1}\right)$ & $2.1 \pm 0.8$ & $3.7 \pm 0.7$ & $2.3 \pm 0.2$ & $3.5 \pm 0.4$ & - $\quad 2.4 \pm 0.8$ & $1.3 \pm 0.4$ \\
\hline & Amplitude (kPa) & $3.4 \pm 0.3$ & $3.7 \pm 0.2$ & $2.6 \pm 0.2$ & $5.5 \pm 0.4$ & $* \quad 3.2 \pm 0.6$ & $2.6 \pm 0.2$ \\
\hline \multirow[t]{2}{*}{ Parameters } & Motility Index & $10.9 \pm 0.5$ & $12 \pm 0.4$ & $10.8 \pm 0.1$ & $12.3 \pm 0.3$ & $* 10.7 \pm 0.6$ & $8.7 \pm 0.2$ \\
\hline & Motility Activity $\left(h^{-1}\right)$ & $12.3 \pm 2.4$ & $20.8 \pm 0.7$ & - $13.4 \pm 1.2$ & $18.6 \pm 0.7$ & * $12.3 \pm 0.7$ & $11.7 \pm 2$ \\
\hline
\end{tabular}

Recordings at Day 0 during 24-h endotoxin or saline infusion and at Day 2 and 5, one and four days after endotoxin infusion, respectively. Enteral nutrition (fed state) started $8 \mathrm{~h}$ after cessation of endotoxin infusion. Abbreviation used: $E T=$ endotoxin. Mean \pm SEM. Statistics by repeated measures ANOVA; all motility parameters showed a significant group effect. No time and no time $x$ group effects were observed. "Significantly different from Control at Day 0,2 or 5 ( $P<0.05$ with Bonferroni correction). No time effects were observed between the fasting and fed state within the Con group.

\section{Analysis of motility recordings}

Motility recordings were analyzed semi-automatically using commercially available software (MMS). The number of pressure waves with an amplitude $\geq 1.4 \mathrm{kPa}$, their mean amplitude and motility index $\left(\mathrm{Ml}=\log _{\mathrm{e}}\right.$ (sum of contraction amplitudes $*$ number of contractions +1$)$ ) were calculated after elimination of artifacts such as abdominal pressure peaks, respiration and drift of the transducers. Motility events were analyzed on the fourth jejunal channel, as the most distant channel from the anastomosis was considered most reliable. The frequency of contractions, the mean amplitude and the mean motility index (MI) were defined for 1-hour periods. Motility activity (MA), was calculated as: $M A=M I *$ number of MMC's $* h^{-1}$. The MMC cycle was defined as the time from the end of phase III of the complex to the end of next phase III as identified by 
the criteria of Code and Marlett (1). The distinct phases of the MMC were determined visually; phase III of the MMC was identified as distally propagating regular contractions preceded by phase II; a period of irregular contractions and followed by phase I; a period of absent contractions. The migration velocity of the MMC's was determined semiautomatically by assessing the time of passage of the end of phase III along at least 2 channels. The mean migration velocity of the MMC was calculated in $\mathrm{mm}$ per minute.

\section{Statistical analysis}

The data were analyzed by repeated measures ANOVA for differences between treatments and differences in time, using as within factor time with 3 levels and as between-factors treatment (+/- endotoxin). When significant time differences appeared, univariate $F$ tests were used for contrasts. Levels of significance were set at $P<0.05$. The independent-samples T-test with Bonferroni correction was performed for group comparisons at Day 0 (motility parameters) or Day 1 (hemodynamic parameters), Day 2 and Day 5.
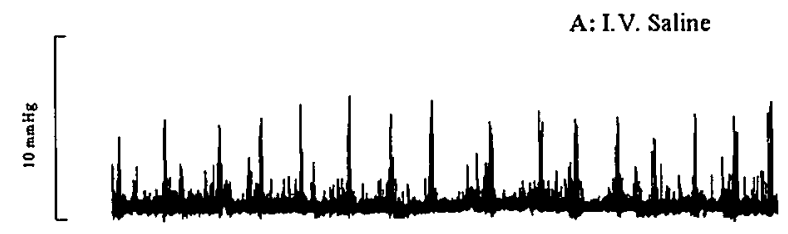

B: I.V. Endotoxin
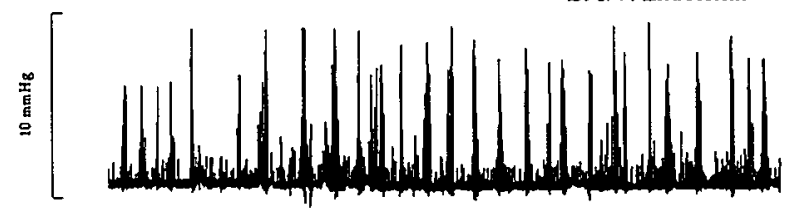

Figure 2. Manometric pressure recording at Day 0 (one channel shown). Fasting jejunal motility pattern in saline-treated control pigs (A) or endotoxin-treated pigs (B). Intravenous Escherichia coli lipopolysaccharide infusion resulted in acceleration of MMC cycling frequency and migration during fasting.

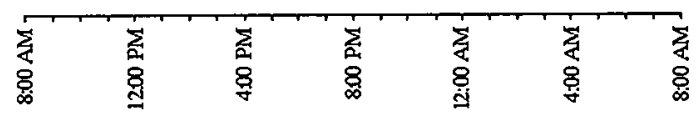

\section{Results}

\section{Effect of enteral feeding}

In both fasted (Figure 2) and fed pigs (Figure 3), a regular MMC pattern was observed in the jejunum during the period of recording. MMC's recorded during fasting and feeding recurred at average intervals of about comparable duration (Table 1). However, in the fasting state the period of quiescence (phase I) occupied about $35 \%(21 \pm 3 \mathrm{~min}$ ) of the total MMC whereas this period almost disappeared during feeding $(4 \pm 3 \mathrm{~min})$. Phase I 
was replaced by phase II-like activity of irregular contractile activity during feeding. Neither the frequency, nor the amplitude of the jejunal contractions observed in phase II in the fed state differed significantly from those observed during fasting (Table 1). Moreover, the average propagation velocity of the MMC cycles remained unaltered by

A: Post-Saline
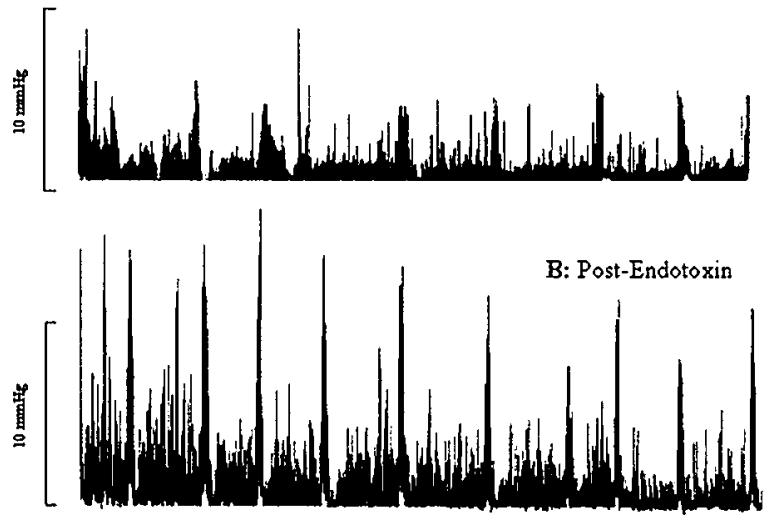

feeding intervention.

Figure 3. Manometric pressure recording at Day 2 (one channel shown). Jejunal motility pattern in pigs during enteral feeding one day after cessation of saline (A) or endotoxin (B). Postendotoxin, changes in jejunal motility pattern were maintained during feeding with an increased contraction frequency and amplitude in addition.

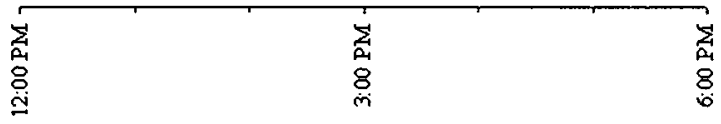

\section{Effect of endotoxin infusion}

At Day 1, the body temperature of the 24-h endotoxin infused pigs (Table 2) was higher than in control pigs. The mean arterial pressure was not affected by endotoxin infusion (Table 2) while the heart rate of the endotoxin-infused pigs was elevated at Day 1. At Days 2 and 5, one and four days after cessation of endotoxin infusion, the body temperature and heart rate were normalized. The blood flow to the portal-drained viscera (Table 2) remained unchanged by endotoxin infusion. Except for body temperature, no statistically significant interactions between time and endotoxin treatment were observed for measured hemodynamic variables. All 6 endotoxin-treated pigs showed no disruption of the regular MMC pattern (Figure 2). However, reduced MMC duration in the endotoxin-treated pigs compared with controls occurred (Table 1) due to shortening and/or disappearance of the irregular contraction phase II (Figure 4). Phase I and phase III duration did not significantly change by endotoxin. All endotoxin-treated pigs supported the intragastric feeding that succeeded the fasting period since vomiting and intragastric retention were not observed. However, the recordings of 3 fasted and 2 fed endotoxin-treated pigs showed retrograde contractions that were of temporary origin $(<1.5 \mathrm{~h})$. The increase in MMC frequency as observed in the fasting state was still present during feeding at 1 day after endotoxin infusion (Figure 3, Table 1). Moreover, 
the migration velocity of the MMC cycles was significantly increased at Day 0 during fasting endotoxemia and MMC phase III occurred almost simultaneously at all 4 recording channels (Figure 4). This increase in MMC migration velocity was maintained at Day 2, 1 day postendotoxin during feeding. While the mean contraction frequency and amplitude in the endotoxin-treated pigs did not differ significantly from controls on the day of endotoxin administration, they both were significantly elevated in the fed state 1 day after pigs had received endotoxin (Table 1). The motility index of the jejunal contractions therefore remained unchanged during endotoxin infusion and increased the following day compared with controls (Table 1). Due to the increased MMC cycle frequency, the motility activity of the endotoxin-treated pigs was higher compared with the saline-treated pigs at Day 0 (Table 1). Motility activity remained relatively high for at least 1 day after endotoxin administration. The changes in motility pattern observed in the endotoxin-treated pigs were accompanied by development of watery diarrhea in these pigs. The motility pattern at 4 days postendotoxin (Day 5) was not significantly different from control values.

Table 2. Hemodynamic parameters and blood flow across the portal-drained viscera in pigs.

\begin{tabular}{lccccccc}
\hline & \multicolumn{5}{c}{ FASTING } & \multicolumn{2}{c}{ DED } \\
\hline & \multicolumn{2}{c}{ Day 0 } & \multicolumn{2}{c}{ Day 2 } & \multicolumn{2}{c}{ Day 5 } \\
\hline & Control & ET & Control & ET & Control & ET \\
\hline Temperature & $37.6 \pm 0.3$ & $39.9 \pm 0.2$ & $* 38.0 \pm 0.2$ & $38.1 \pm 0.3$ & $37.5 \pm 0.4$ & $38 \pm 0.4$ \\
MAP & $78 \pm 10$ & $80 \pm 10$ & $77 \pm 7$ & $78 \pm 8$ & $78 \pm 7$ & $78 \pm 9$ \\
HR & $88 \pm 5$ & $119 \pm 13$ & $80 \pm 7$ & $82 \pm 10$ & $84 \pm 7$ & $82 \pm 5$ \\
PDV blood flow & $35 \pm 4$ & $43 \pm 2$ & $45 \pm 6$ & $53 \pm 9$ & $41 \pm 9$ & $39 \pm 3$ \\
\hline
\end{tabular}

Recordings at Day 1 during 24-h endotoxin or saline infusion and at Day 2 and 5, one and four days after endotoxin infusion, respectively. Enteral nutrition (fed state) started $8 \mathrm{~h}$ after cessation of endotoxin infusion. Abbreviation used: $\mathrm{ET}=$ endotoxin. Mean $\pm \mathrm{SEM}$. Temperature in ${ }^{\circ} \mathrm{C}, \mathrm{MAP}$ in $\mathrm{mmHg}, \mathrm{HR}$ in beats $/ \mathrm{min}$ and flow in $\mathrm{mL} \bullet \mathrm{kg} \mathrm{bw}{ }^{-1} \bullet \mathrm{min}^{-1}$. Statistics by repeated measures ANOVA; temperature and HR showed a significant group effect. A time-dependent effect of ET was observed on temperature. No time effect was observed. No differences were observed between the fasting and the fed state within the Control group. ${ }^{\star} \mathrm{P}<0.016$; independent samples T-test with Bonferroni correction: significant difference between the treatment groups at Day 0,2 or 5 .

\section{Discussion}

In this model of endotoxemia, the continuous infusion of endotoxin evoked an acceleration of MMC migration and MMC cycling frequency in the jejunum. Moreover, one day after endotoxin challenge, a pronounced increase in the motility index due to increased contraction frequency and amplitude was manifest. Endotoxin infusion also resulted in increased motility activity of the jejunum that lasted until at least one day after 
endotoxin infusion during enteral feeding. Normal motility was restored after 4 days of feeding.

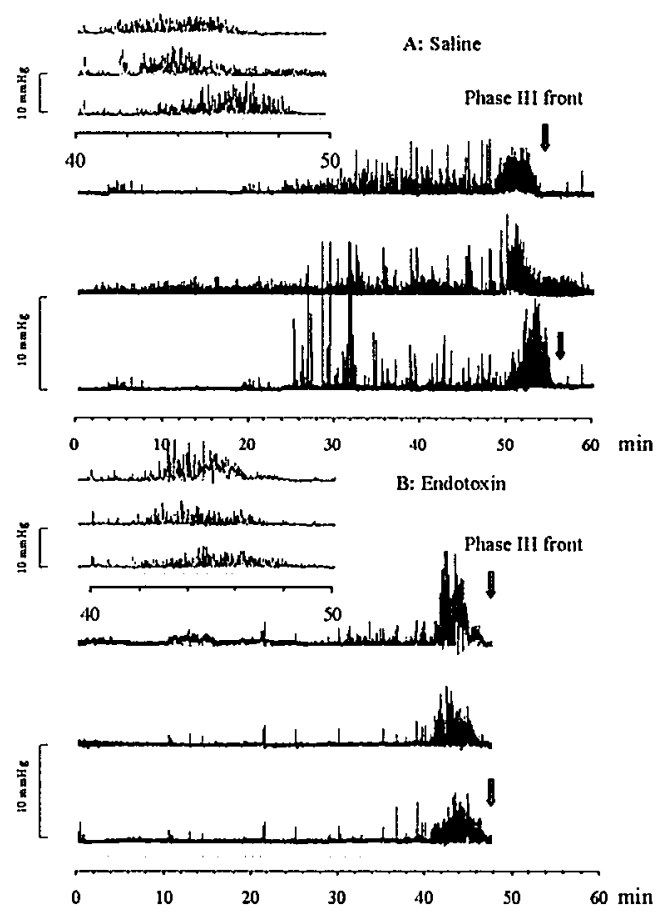

Figure 4. Manometric pressure recording of one MMC cycle (three jejunal channels shown) in the jejunum of fasting saline-treated control pigs (A) or endotoxin-treated pigs (B). The upper left corner shows phase III in more detail.

Many of the endotoxemia models studying gastrointestinal motility disturbances use large single doses of endotoxin reproducing hemodynamic alterations that resemble hypodynamic circulatory shock rather than hyperdynamic sepsis. In the current endotoxemia model, pigs were challenged with a continuous low dose of endotoxin accompanied by fluid support to imitate compensated sepsis reproducing a sepsis model more representative of modern intensive care (6). One of the fundamental features of resuscitation during slow endotoxin infusion is the mesenteric blood flow, which is, as in the case of our pigs, maintained $(9,15)$. Moreover, the 24-h endotoxin treated pigs exhibited a hyperdynamic cardiovascular and metabolic profile including elevated body temperature and heart rate and preserved mean arterial pressure. We conclude that this model reproduces several of the systemic hemodynamic characteristics of the sepsis syndrome in humans.

To imitate clinical sepsis in this model, endotoxin infusion was followed by enteral feeding as enteral nutrition often is indicated in septic patients with a protracted disease course. All enterally fed pigs supported the enteral nutrition as no vomiting and gastric retention was observed. Generally, when fed one large meal the characteristic interdigestive repetitious MMC pattern is disrupted and replaced for postprandial irregular contractions. However, studies in pigs that were fed ad lib, showed that the basic MMC pattern persisted regardless of feeding (22). Accordingly, continuous enteral feeding preserved the MMC pattern in our pigs, although the quiescence period (phase I) was absent due to an increased phase II to phase I ratio. The observation that MMC cycles were maintained during enteral feeding might be due to the fact that, like in humans, the occurrence of MMC's is preserved when only small amounts of nutrition are continuously 
infused (21). The MMC cycling frequency in the proximal jejunum was similar during fasting and enteral feeding and showed a cycle duration of 54-60 min that is comparable to previously reported values for fasted pigs of about the same age (20).

Hyperdynamic endotoxemia exerted pronounced and transient effects on jejunal motility. The induced changes included increased frequency of MMC cycling due to shortening or even disappearance of phase II both during endotoxin and one day thereafter during feeding 1 day post-endotoxin. A comparable increase in MMC cycling was reported in the jejunum of piglets that were bolus-injected with endotoxin (10). Although the MMC cycle in these pigs initially completely disappeared, the MMC cycling pattern of our pigs did not disappear. Possibly the maintenance of visceral flow, due to supportive fluid support, contributed importantly to the preservation of peristalsis and MMC cycling in the jejunum. The increase in MMC cycle frequency during and one day after endotoxin infusion was paralleled by acceleration of the MMC phase III migration along the jejunum. This increased jejunal phase III migration may contribute to rapid transit of small bowel content. Rapid gastrointestinal transit was also observed in dogs $(2,4)$ challenged with a single dose of $E$. coli endotoxin at one day postendotoxin, with simultaneously increased motility indexes in the colon. In addition to changes in the $M M C$ pattern, one day postendotoxin the jejunal contractions were more intensified and characterized by increased amplitude and frequency and subsequent higher motility index. Increased jejunal motility index in this porcine model of endotoxemia contrasts with decreased motility index shown in a canine model of endotoxemia (3). The differences observed in jejunal motility indexes possibly rely upon differences in model and animal species used.

Although symptoms of large bowel dysmotility in sepsis caused by gram-negative endotoxins are relatively well recognized, small intestinal disturbances are less well understood. E. coli endotoxin is an important stimulus involved in the pathogenesis of secretory diarrhea $(13,14,19)$ which is characterized by accelerated gastrointestinal transit, notably through the colon. The marked increase in propulsive activity in the jejunum due to an increased incidence of propagated contractions may have caused accelerated small intestinal transit to such a degree that it resulted in diarrhea. In patients suffering from secretory diarrhea, similar spike burst complexes with increased incidence are present in the small bowel (25). Moreover, characteristics of increased motility activity and accelerated MMC migration in the jejunum of our continuously endotoxin-infused pigs resemble colonic motility alterations in $E$. coli endotoxin-treated dogs (2). It, however, remains to be established whether jejunal motility changes are comparable or occur simultaneously with colonic motility changes that are observed in the pathogenesis of endotoxin-induced diarrhea. 
From this study it can be concluded that, although a hemodynamic profile characteristic of the hyperdynamic state was present, it has to be taken into account that this experimental model of endotoxemia induced by continuous endotoxin infusion, may not necessarily reproduce all the features representative of the human compensated sepsis profile with all complications. This hyperdynamic endotoxemia model reveals that the jejunal motility disorders in the response to endotoxin infusion include increased MMC migration velocity and increased MMC cycling frequency with absence or shortened phase II. These changes were still manifest one day postendotoxin during feeding, indicating a protracted effect of endotoxin challenge whereas the MMC pattern was restored 4 days postendotoxin. Acceleration of jejunal MMC migration velocity may contribute to increased transit of jejunal contents, which would be supported by the observation that our pigs developed diarrhea. The jejunal manometric recordings of this prolonged pig model of endotoxemia point out that MMC cycles are preserved during mild endotoxemia and in the postendotoxemic and fed condition. Similar endotoxininduced changes in jejunal motility along the small bowel may contribute to the complications manifest in critically ill patients suffering endotoxin-induced diarrhea.

\section{Acknowledgements}

The authors acknowledge Mrs. G.A.M. ten Have for assistance during experiments. This study was supported by grant 902-23-098 from the Netherlands Organization for Scientific Research.

\section{References}

1. Code, C. F., and J. A. Marlett. The interdigestive myo-electric complex of the stomach and small bowel of dogs. $J$ Physiol (Lond) 246: 289-309, 1975.

2. Cullen, J. J., D. K. Caropreso, and K. S. Ephgrave. Effect of endotoxin on canine motility and transit. $J$ Surg Res 58: 90-95, 1995.

3. Cullen, J. J., D. K. Caropreso, K. S. Ephgrave, L. L. Hemann, and M. M. Hinkhouse. The effect of endotoxin on canine jejunal motility and transit. J Surg Res 67: 54-7, 1997.

4. Cullen, J. J., D. Mercer, M. Hinkhouse, K. S. Ephgrave, and J. L. Conklin. Effects of endotoxin on regulation of intestinal smooth muscle nitric oxide synthase and intestinal transit. Surgery 125: 339-44, 1999.

5. Eskandari, M. K., J. C. Kalff, T. R. Billiar, K. K. Lee, and A. J. Bauer. LPS-induced muscularis macrophage nitric oxide suppresses rat jejunal circular muscle activity. Am J Physiol 277: G478-86, 1999.
6. Fink, M. P., and S. O. Heard. Laboratory models of sepsis and septic shock. J Surg Res 49: 186-196, 1990.

7. Hellstrom, P. M., A. al-Saffar, T. Ljung, and E. Theodorsson. Endotoxin actions on myoelectric activity, transit, and neuropeptides in the gut. Role of nitric oxide. Dig Dis Sci 42: 1640-51, 1997.

8. Knapik, Z. Motility disturbances of the large intestine. Z Gesamte Inn Med 36: suppl 194-6, 1981.

9. Kreimeier, U., L. Frey, J. Dentz, T. Herbel, and K. Messmer. Hypertonic saline dextran resuscitation during the initial phase of acute endotoxemia: effect on regional blood flow. Crit Care Med 19: 801-9, 1991.

10. Li, J. X., J. R. Oliver, and J. B. Philips, 3rd. Endotoxin induces biphasic alterations in small intestinal myoelectric activity in fasted newborn piglets. Pediatr Res 40: 822-6, 1996.

11. Mainous, M. R., E. F. Block, and E. A. Deitch. Nutritional support of the gut: how and why. New Horiz 2: 193-201, 1994. 
12. Martinez-Cuesta, M. A., M. D. Baracchina, B. Beltran, S. Calatayud, and J. Esplugues. Nitric oxide modulates the acute increase of gastrointestinal transit induced by endotoxin in rats: a possible role for tachykinins. J Pharm Pharmacol 49: 988-990, 1997.

13. Meyer, W. Clinical aspects, pathogenesis and therapy of shock due to sepsis caused by gram-negative organisms. Schweiz Med Wochenschr 96: 137-43, 1966.

14. Moon, H. W. Pathogenesis of enteric diseases caused by Escherichia coli. Adv Vet Sci Comp Med 18: 179-211, 1974.

15. Murphey, E. D., and D. L. Traber. Cardiopulmonary and splanchnic blood flow during 48 hours of a continuous infusion of endotoxin in conscious pigs: a model of hyperdynamic shock. Shock 13: 224-9, 2000.

16. Nieuwenhuijs, V. B., A. Verheem, H. van Duijvenbode-Beumer, M. R. Visser, J. Verhoef, H. G. Gooszen, and L. M. Akkermans. The role of interdigestive small bowel motility in the regulation of gut microflora, bacterial overgrowth, and bacterial translocation in rats. Ann Surg 228: 188-93, 1998.

17. Nissan, A., J. M. Zhang, Z. Lin, Y. Haskel, H. $R$. Freund, and M. Hanani. The contribution of inflammatory mediators and nitric oxide to lipopolysaccharide-induced infussusception in mice. J Surg Res 69: 205-7, 1997.

18. Pons, L., M. T. Droy-Lefaix, P. Braquet, and L. Bueno. Role of free radicals and plateletactivating factor in the genesis of intestinal motor disturbances induced by Escherichia coli endotoxins in rats. Gastroenterology 100: 94653, 1991.
19. Powell, D. W. Approach to the Patient with Diarrhea. In: Textbook of Gastroenterology, edited by $T$. Yamada. Philadelphia: JB Lippincott Company, 1995, p. 813-840.

20. Rayner, V., and g. Wenham. Small intestinal motility and transit by electromyography and radiology in the fasted and fed pig. $J$ Physiol 379: 245-256, 1986.

21. Riachi, G., P. Ducrotte, C. Guedon, C. Bouteloup, P. Denis, R. Colin, and $E$. Lerebours. Duodenojejunal motility after oral and enteral nutrition in humans: a comparative study. J Parenter Enteral Nutr 20: 150-155, 1996.

22. Ruckebusch, Y., and L. Bueno. The effect of feeding on the motility of the stomach and small intestine in the pig. Br $J$ Nutr 35: 397 405, 1976.

23. Ten Have, G. A. M., M. C. F. Bost, J. C. A. W. Suyk-Wierts, A. E. J. M. van den Bogaart, and N. E. P. Deutz. Simultaneous measurement of metabolic flux in portally-drained viscera, liver, spleen, kidney and hindquarter in the conscious pig. Lab Anim 30: 347-358, 1996.

24. Thompson, J. S. The intestinal response to critical illness. Am J Gastroenterol 90: 190200, 1995.

25. Vantrappen, G., J. Janssens, G. Coremans, and R. Jian. Gastrointestinal motility disorders. Dig Dis Sci 31: 5S-25S, 1986.

26. Wirthlin, D. J., J. J. Cullen, S. T. Spates, J. L. Conklin, J. Murray, D. K. Caropreso, and K. S. Ephgrave. Gastrointestinal transit during endotoxemia: the role of nitric oxide. $J$ Surg Res 60: 307-11, 1996. 

EFFECTS OF LONG-TERM INTRAVENOUS AND INTRAGASTRIC L-ARGININE INTERVENTION ON JEJUNAL MOTILITY AND VISCERAL NITRIC OXIDE PRODUCTION IN THE ENDOTOXEMIC PIG

\author{
Maaike J Bruins \\ Yvette C Luiking \\ Peter B Soeters \\ Nicolaas EP Deutz
}

Department of Surgery, Maastricht University, The Netherlands 


\section{Abstract}

Nitric oxide $(\mathrm{NO})$ is involved in the regulation of small intestinal motility through its mediator function in gut inhibitory nitrergic neurons. This study aimed to examine the effects of intervention with L-arginine, as an exogenous substrate for NO, on the motility pattern under endotoxemic conditions in relation to visceral NO synthesis.

Endotoxemia was induced by $24-\mathrm{h}$ infusion of Escherichia coli endotoxin $(3 \mu \mathrm{g} \cdot \mathrm{kg}$ $b^{-1} \cdot h^{-1}$, i.v.). Nonendotoxemic pigs received saline (Day 0 ). Endotoxin-treated pigs were intravenously infused with either L-arginine or L-alanine. All pigs received fluid resuscitation. After cessation of endotoxin infusion, intravenous L-arginine or L-alanine infusion was continued intragastricly as a supplement in the enteral diet until Day 2. Jejunal motility was recorded on Days 0 and 2 by manometry. NO production was measured using stable isotope techniques.

Intravenous administration of L-arginine during endotoxin infusion restored the endotoxininduced increase in frequency and velocity of jejunal migrating motor complex (MMC) cycles to control values. Intragastric infusion of L-arginine on Day 2 after endotoxin had similar effects. The by endotoxin-induced enhanced NO production in the portal-drained viscera on Day 1 was further increased by intravenous L-arginine infusion. On Day 2 after endotoxin treatment visceral NO synthesis no longer differed from controls, but, was enhanced by intragastric L-arginine treatment.

Restoration of endotoxin-induced increased motility activity by L-arginine coincided with improved visceral NO synthesis. This suggests a possible therapeutic role of exogenous L-arginine in the management of hypermotility associated with sepsis.

\section{Introduction}

Impaired gastrointestinal function such as malabsorption and disruption of normal gastrointestinal motility are frequently observed complications in sepsis. Frequently observed intestinal disorders in critically ill patients include retardation or acceleration of gastrointestinal transit associated with generalized ileus or diarrhea (23). In septic patients, many factors such as anesthesia, surgical manipulation, infection, therapeutic agents and nutritional status will influence their intestinal motility (23). The complex interaction between the many exogenous and endogenous mediators implicated in sepsis determines the picture of a disturbed motility pattern. Endogenous bacterial endotoxins and endotoxin-induced mediators like neuropeptides, cytokines and nitric oxide (NO) are principal stimuli involved in the pathogenesis of motility disorders observed in endotoxin-induced septicemia (16-18). 
Depending on the quantities in which it is formed, NO has a dual effect on small intestinal motility. Excessive production of NO by the NO synthase-2 (NOS-2) isoform during inflammation is associated with motility disturbances $(9,24)$. Synthesized in small amounts by the constitutive NOS-1 enzyme, NO is an important neurotransmitter of inhibitory non-adrenergic non-cholinergic (NANC) neurons of the gastrointestinal tract, and is therefore one of the main regulators of small intestinal motility (20). NANC neurons containing NO mediate smooth muscle relaxation $(3,14)$, inhibit slow wave amplitude and frequency (3) and coordinate the periodic cycling of the migrating motor complexes (MMC's) in the gut $(19,22)$. Moreover, administration of the NO synthase (NOS) inhibitor NG-nitro-L-arginine attenuated NANC-mediated smooth muscle relaxation in the rat small intestine $(3,13)$ suggesting a basic requirement for $\mathrm{NO}$ as a NANC inhibitory mediator in small intestinal motility. Prolonged septicemia is often accompanied by decreased plasma L-arginine levels $(2,12)$, which may become limiting for NO synthesis by NOS-1 enzyme in the intestinal NANC nerves. Speculating that Larginine is involved in the control of the periodic MMC cycling through NOS-mediated NO production, we investigated the impact of L-arginine supplementation on the motility pattern in a long-lasting model of endotoxemia.

The aim of the present study, therefore, was to assess the effects of L-arginine supplementation under conditions of acute and prolonged endotoxemia on jejunal motility. For that purpose, pigs were continuously infused with low doses of endotoxin, given fluid support and treated with either intravenous $L$-arginine or L-alanine infusion. To determine the effects of L-arginine intervention in combination with enteral feeding on jejunal motility L-arginine or L-alanine was infused intragastricly as a supplement in the diet after cessation of endotoxin challenge.

\section{Materials and methods}

\section{Animals}

Twenty-one female crossbred pigs (Yorkshire $x$ Dutch Landrace) weighted: $20-22 \mathrm{~kg}$ body weight (bw). Pigs were given $1 \mathrm{~kg}$ of regular pig feed once a day (Landbouwbelang, Roermond, the Netherlands), sustaining a growth rate of about $300 \mathrm{~g}$ per day. The Animal Ethics Committee of the Maastricht University approved the study.

\section{Surgical procedure}

All pigs were fasted when surgery was performed. Pigs were intubated and anesthesia maintained with a mixture of $\mathrm{N}_{2} \mathrm{O} / \mathrm{O}_{2}(1: 2)$ and halothane $(0.8 \%)$. As antimicrobial prophylaxis, $6.25 \mathrm{mg} / \mathrm{kg}$ bw lincomycin (Lincomycin, A.U.V., Cuyk, The Netherlands) and 
$12.5 \mathrm{mg} / \mathrm{kg}$ bw spectinomycin (Spectinomycin, A.U.V.) was intravenously administered. To avoid coagulation and as for postoperative anaesthetic, flunixine (Finadyne, ScheringPloegh, Brussel, Belgium) was given intravenously. After a midline abdominal incision was made, $80-\mathrm{cm}$ long Tygon catheters (ID $1.6 \mathrm{~mm}$, OD $2.8 \mathrm{~mm}$, Tygon, Westvaco, Cleveland, $\mathrm{OH}$, USA) for blood sampling were placed in the abdominal aorta, just above the bifurcation, and in the portal vein. Catheters for infusion of endotoxin and for paraaminohippuric acid (PAH) were inserted in the inferior caval vein just above the right renal vein and one in the splenic vein, respectively. A gastric tube (I.D. $1.6 \mathrm{~mm}$, O.D. 3.2 $\mathrm{mm}$, Tygon) was placed in the stomach. All catheters were tunneled through the abdominal wall and skin. A Bishop-Koop stoma was constructed by transecting the jejunum $20 \mathrm{~cm}$ distal to the ligament of Treitz (the transition of duodenum to jejunum). The anatomical continuity of the remaining bowel was reestablished by an end-to-side anastomosis (Figure 1) made with a single layer running suture (PDS 4-0 synthetic polydioxanon; Ethicon, Norderstedt, Germany). A filled balloon catheter (Size Ch.16, 30$50 \mathrm{ml}$; Rüsch-Gold, Kernen, Germany) served to close the stoma and to avoid leakage of jejunal content. Pigs wore a canvas harness to protect the catheters and stoma and to allow easy handing. The first two postoperative days pigs received $6.25 \mathrm{mg} / \mathrm{kg} \mathrm{bw}$ lincomycin and $12.5 \mathrm{mg} / \mathrm{kg}$ bw spectinomycin intravenously twice daily (23). To get accustomed to the experimental condition, the pigs were repeatedly placed in a movable cage.

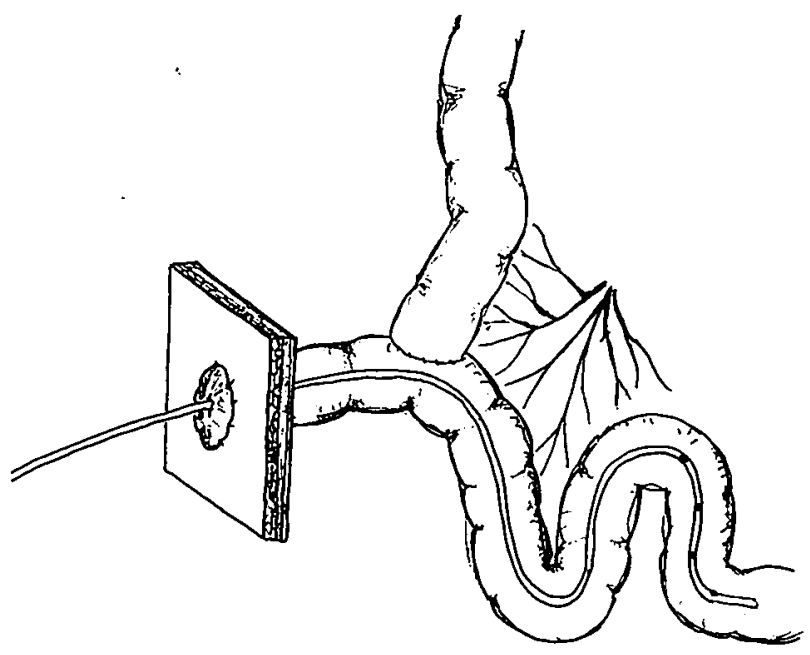

Figure 1. Schematic drawing of the surgical jejunostomy for the purpose of catheter insertion according to the end-to-side single-layer anastomotic closure. 


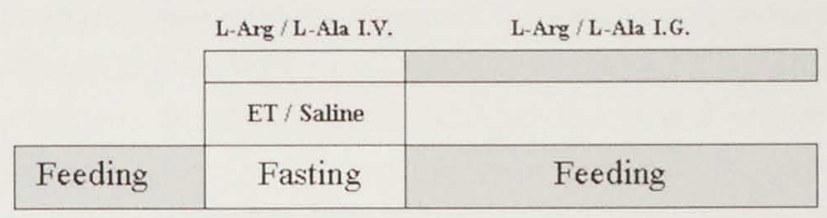

Day

$-1$

0

Motility recording

Blood sample

Figure 2. Schematic illustration of the experimental design. During Day -1 , pigs are intragastricly fed, during Day 0 pigs are 24-h fasted and infused saline or endotoxin (ET), during Day 1 and 2, pigs are intragastricly fed. Pigs are administered saline (Con) or endotoxin with either L-Ala (ET-Con) or L-Arg intervention (ETArg). During Day 0 L-Ala/L-Arg is intravenous (I.V.) infused, whereas during Day 1 and 2 L-Ala/L-Arg is intragastricly (I.G.) infused. Jejunal pressure recordings are performed by manometry at Day 0 during $24-h$ and at Day 2 during 8-h. Arterial, portal, splenic and caval vein blood samples are collected at Day 1 and Day 2, 1 hour after stable isotope and para-aminohippuric acid infusion.

\section{Experimental protocol}

From the tenth postoperative day onwards, pigs were intragastricly fed a high-protein liquid diet (Nutrison Steriflo Protein-Plus, Nutricia B.V., Zoetermeer, the Netherlands). Continuous infusion of the meal into the gastrostomy catheter via a swivel system allowed the pigs to move freely. Infusion of intragastric nutrition was started three days prior to the experiment at a rate of $4 \mathrm{~mL} \cdot \mathrm{kg} \mathrm{bw}^{-1} \cdot \mathrm{h}^{-1}\left(\approx 0.3 \mathrm{~g} \cdot \mathrm{kg} \mathrm{bw}^{-1} \cdot \mathrm{h}^{-1}\right.$ of protein) to get the pigs accustomed to the diet. Three out of 21 pigs that showed no periodic MMC cycling at the start of the experiment were excluded from the study. Pigs with a regular motility pattern were randomly assigned to the control group ( $n=6$, Con group), the endotoxin control group receiving L-alanine ( $n=6$, ET-Con group) or the L-arginine treatment group ( $n=6, E T$-Arg group). Experiments were started after an 8 - $h$ fasting period at Day 0. The design of the protocol is delineated in Figure 2. At Day 0, endotoxin (lipopolysaccharide (LPS) from Escherichia coli, serotype 055:B5; Sigma Chemical Co., St. Louis, MO, USA) dissolved in $150 \mathrm{mmol} / \mathrm{L} \mathrm{NaCl}$ (saline) was intravenously infused at a rate of $3 \mu \mathrm{g} \cdot \mathrm{kg} \mathrm{bw}^{-1} \cdot \mathrm{h}^{-1}$ via the caval vein catheter during $24 \mathrm{~h}$ whereas control pigs received saline at an equal rate. The endotoxin-treated pigs were either intravenously administrated L-arginine (iso-osmolar, $5.3 \mu$ moløkg bw ${ }^{-1} \bullet \mathrm{min}^{-1}, \mathrm{pH} 7.3$; ET-Arg group) or $\mathrm{L}$-alanine as a control amino acid at an isocaloric rate $\left(10.6 \mu \mathrm{mol} \bullet \mathrm{kg} \mathrm{bw}^{-1} \bullet \mathrm{min}^{-1}, \mathrm{pH} 7.3\right.$; ET-Con group). To imitate a hyperdynamic profile characteristic of human compensated sepsis with preserved intravascular volume and blood flow to the organs (11), all pigs received extra saline $\left(20 \mathrm{~mL} \cdot \mathrm{kg} \mathrm{bw}^{-1} \cdot \mathrm{h}^{-1}\right.$, intravenously) during $24 \mathrm{~h}$. Immediately after the start of endotoxin or saline infusion, the manometry catheter was inserted through the jejunal stoma and motility recordings started for 24 hours (Day 0). After $24 \mathrm{~h}$ at Day 
1 , infusions and recordings were discontinued and intragastric nutrition started $(4 \mathrm{~mL} \cdot \mathrm{kg}$ $\left.\mathrm{bw}^{-1} \cdot \mathrm{h}^{-1}\right)$. The intravenous L-arginine or L-alanine infusion was succeeded at an equal infusion rate through the gastrostomy catheter as a supplement in the enteral meal. The following day (Day 2), 1 day after cessation of the endotoxin or saline infusion, 8-h motility readings from the fed state were obtained from Con, ET-Con and ET-Arg pigs. Also at Day 1 and Day 2 (at $0 \mathrm{~h}$ ), blood samples were collected to measure NO synthesis rate by the portal-drained viscera during and 1 day after endotoxin challenge, respectively.

\section{NO synthesis measurements}

To measure NO production by the portal-drained viscera at Day 1 and Day 2, paraaminohippuric acid (PAH, 25 mmol/L, A 1422, Sigma Chemicals Co., St. Louis, MO, USA) and stable isotope (Mass Trace, Woburn, MA, USA) infusion protocols were performed. Steady state conditions for $\mathrm{PAH}$ and stable isotopes were previously assessed in a pilot experiment in which blood samples were collected in triplicate at $10-$ min intervals (data not shown). Steady state was then confirmed to be reached within 60 $\mathrm{min}$ at the different blood sampling sites (21). Therefore, blood samples were coliected 1 $\mathrm{h}$ after the start of PAH and isotope infusions and, subsequently, $\mathrm{PAH}$ and isotope infusions stopped. Background blood samples were collected before the start of the PAH and isotope infusions. The PAH infusion was started at a rate of $40 \mathrm{~mL} / \mathrm{h}$ through the splenic vein catheter after an initial prime of $5 \mathrm{~mL}$. Simultaneously, a primed and constant infusion of L-[guanidino- ${ }^{15} \mathrm{~N}_{2}$ ]arginine $\left({ }^{15} \mathrm{~N}_{2}\right.$-arginine; prime: $3.2 \mu \mathrm{mol} \cdot \mathrm{kg} \mathrm{bw}{ }^{-1}$, infusion: $\left.6.4 \mu \mathrm{mol} \bullet \mathrm{kg} \mathrm{bW}^{-1} \cdot \mathrm{h}^{-1}\right)$ and $\mathrm{L}$-[ureido- ${ }^{13} \mathrm{C} ; 5,5-{ }^{2} \mathrm{H}_{2}$ ]citrulline $\left(\mathrm{C}_{1} \mathrm{D}_{2}\right.$-citrulline; prime: $1.5 \mu \mathrm{mol} \bullet \mathrm{kg} \mathrm{bw}^{-1}$, infusion: $0.6 \mu \mathrm{mol} \bullet \mathrm{kg} \mathrm{bw}^{-1} \cdot \mathrm{h}^{-1}$ ) was started via the caval vein catheter.

\section{Sample processing}

Immediately after sampling, blood was distributed in pre-chilled, heparinized tubes (Sarstedt, Nümbrecht, Germany) on ice. For PAH determinations, $300 \mu \mathrm{L}$ of whole blood was added to $600 \mu \mathrm{L}$ of $0.7 \mathrm{mmol} / \mathrm{L}$ trichloroacetic acid (TCA; Merck, Darmstadt, Germany) and thoroughly mixed. After centrifugation at $4^{\circ} \mathrm{C}$ for $5 \mathrm{~min}$ at $8,500 \mathrm{~g}, 500 \mu \mathrm{L}$ of plasma was deproteinized by mixing with $20 \mathrm{mg}$ dry sulfosalicylic acid. After centrifuging at $4^{\circ} \mathrm{C}$ for $5 \mathrm{~min}$ at $8,500 \mathrm{~g}$ supernatants were collected and stored at $-80^{\circ} \mathrm{C}$ until analysis.

\section{Biochemical analysis}

Whole blood PAH concentration was measured by standard enzymatic methods, using commercially available kits as described previously (12). PAH was detected spectrophotometrically on an automated analysis system (Cobas Mira-S, Hoffmann-La 
Roche, Basel, Switzerland). Amino acid concentrations and enrichments were determined by a fully automated liquid chromatography-mass spectrometry (LC-MS, Thermoquest LCQ, Veenendaal, the Netheriands) system after pre-column derivatization with o-phthaldialdehyde (21). Enrichments were calculated as tracer-to-tracee ratios (TTR).

\section{Calculations}

The $C_{1} D_{2}$-citrulline isotope was used to calculate the whole-body rate of appearance of citrulline ( $\mathrm{Q} \mathrm{Ra} \mathrm{CIT}_{\mathrm{T}}$ in $\left.\mu \mathrm{mol} \cdot \mathrm{kg} \mathrm{bw}^{-1} \cdot \mathrm{min}^{-1}\right)$. Q Ra $\mathrm{RIT}_{\mathrm{T}}$ is calculated in formula (1) by dividing the rate of tracer infused $\left(I\right.$, in $\left.\mu \mathrm{mol} \cdot \mathrm{kg} \mathrm{bw}^{-1} \cdot \mathrm{min}^{-1}\right)$ by the tracer-to-tracee ratio of $\mathrm{C}_{1} \mathrm{D}_{2^{-}}$ citrulline in the arterial plasma (TTR $\mathrm{A}_{\mathrm{C} 1 \mathrm{D2}-\mathrm{CIT}}$ ) (22). TTR values were corrected for background values obtained before start of infusions.

$$
Q_{R_{C I T}}=1 / \text { TTR }_{A N 1-C I T}
$$

Formula (2) was used to calculate the rate of whole-body synthesis of NO (Q No) based upon the plasma isotope transfer from $\mathrm{N}_{2}$-arginine to $\mathrm{N}_{1}$-citrulline.

(2) $Q_{\text {NO }}=Q_{R_{C I T}} *\left(T_{T R} R_{\text {N1-CIT }} / T_{T R}\right.$ A N2-ARG $)$

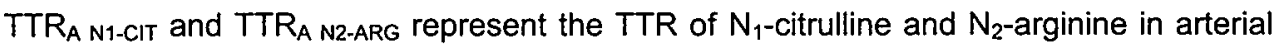
plasma, respectively.

The PDV is defined as the total of all portal-drained organs which, in addition to the spleen and pancreas, largely represents the intestines. To calculate plasma [PAH], blood $[\mathrm{PAH}]$ is corrected for hematocrit values (3). Calculations on plasma flow rate $(\mathrm{mL} \cdot \mathrm{kg}$ bw ${ }^{1} \cdot \mathrm{min}^{-1}$ ) across the portal-drained viscera are based on the principle of indicator dilution methods. By sampling arterially and portally, dilution can be calculated using formula (4) in which I represents the rate of the PAH infused $\left(\mathrm{nmol} \cdot \mathrm{kg} \mathrm{bw}^{-1} \cdot \mathrm{min}^{-1}\right)$ in the splenic vein, entering the portal vein. $[\mathrm{PAH}]_{\mathrm{P}}$ and $[\mathrm{PAH}]_{\mathrm{A}}$ represent the concentration $(\mu \mathrm{mol} / \mathrm{L})$ of $\mathrm{PAH}$ in the portal and arterial plasma, respectively. The dilution of PAH infused via the splenic vein entering the portal vein, depends on the blood flow rate through the portal-drained viscera.

$$
[\mathrm{PAH}]_{\mathrm{PLASMA}}=[\mathrm{PAH}]_{\mathrm{BLOOD}} * 100 /(100-\text { hematocrit })
$$

(4) Flow PLASMA $=\mathrm{I} /\left([\mathrm{PAH}]_{V}-[\mathrm{PAH}]_{\mathrm{A}}\right)$

The rate of NO synthesis by individual organs (Syn No) can be calculated from conversion of $\mathrm{N}_{2}$-arginine to $\mathrm{N}_{1}$-citrulline across the PDV. The term $\mathrm{nb} \mathrm{N}_{\mathrm{N} 1 \text {-CIT }}$ represents the tracer net balance of the $N_{1}$-citrulline isotope in the arterial and portal plasma, respectively. Because of possible fractional extraction of citrulline and, therefore, $N_{1}$ citrulline $\left(\mathrm{FE}_{\mathrm{CIT}}\right.$ ) by the organ, the $\mathrm{nb}{ }_{\mathrm{N} 1-\mathrm{CIT}}$ must be corrected for the loss of $\mathrm{N}_{1}$-citrulline tracer across the organ, which is possible by using the $C, D_{2}$-citrulline isotope in equation 
(5). The term (1 - FE) represents the fraction of total amino acid that bypasses metabolism in the organ and so appears in the output and is used for correction of the $N_{1}$-citrulline $n b(6)$. The organ Syn No is derived from formula (7), in which TTR T N2-ARG represents the arterial $\mathrm{N}_{2}$-arginine tracer to tracee ratio. The TTR of $\mathrm{N}_{2}$-arginine in the arterial plasma free pool was assumed to be the same as the TTR of $\mathrm{N}_{2}$-arginine in the direct precursor pool of NO synthesis.

(5) $\left.\quad \mathrm{FE}_{\mathrm{CIT}}=\left(\mathrm{TTR}_{\mathrm{A}} *[\mathrm{~A}]-\mathrm{TTR}_{\mathrm{V}} *[\mathrm{M}]\right) / \mathrm{TTR}_{\mathrm{A}} *[\mathrm{~A}]\right)_{\mathrm{C1D} 2-\mathrm{CIT}}$

$$
\mathrm{nb}_{\mathrm{N} 1-\mathrm{CIT}}=\text { flow }^{*}\left\{[\mathrm{~V}] * \mathrm{TTR}_{\mathrm{V}}-[\mathrm{A}] * \mathrm{TTR}_{\mathrm{A}} *\left(1-\mathrm{FE}_{\mathrm{CIT}}\right)\right\}_{\mathrm{N} 1-\mathrm{CIT}}
$$

$$
\text { Syn }_{\text {NO }}=\mathrm{nb}_{\mathrm{N1} 1-\mathrm{CIT}} / \mathrm{TTR}_{\mathrm{AN2} \text {-ARG }}
$$

\section{Intestinal motility measurements}

During experiments, motility was studied by ambulatory manometry, using a 4 channel catheter with 4 solid-state pressure transducers (CTO-6, Dunvegan, Isle of Skye, Scotland) spaced $5-\mathrm{cm}$ apart. The transducers were calibrated before the investigations were started. Immediately following start of the infusions, the manometry catheter was inserted into the bowel lumen of the jejunum via the Bishop-Koop jejunostomy, with the pressure transducers located at $10,15,20$ and $25 \mathrm{~cm}$ distal to the end-to-side anastomosis (Figure 1). Pressure signals were sampled at a frequency of $8 \mathrm{~Hz}$ and stored in a data logger (UPS-2020, MMS, Enschede, the Netherlands). A personal computer served as on-line monitoring and retrieval system. Recordings were performed for a period of $24-h$ (fasting) or 8-h (postprandial).

\section{Analysis of motility recordings}

Motility recordings were analyzed semi-automatically using commercially available software (MMS). The number of pressure waves with an amplitude $\geq 1.4 \mathrm{kPa}$, their mean amplitude and motility index $\left(\mathrm{Ml}=\log _{\mathrm{e}}\right.$ (sum of contraction amplitudes $\mathrm{x}$ number of contractions +1$)$ ) were calculated after elimination of artifacts such as abdominal pressure peaks, respiration and drift of the transducers. Motility events were analyzed on the fourth jejunal channel, as the most distant channel from the anastomosis was considered most reliable. The frequency of contractions, the mean amplitude and the mean motility index (MI) were defined for 1 -hour periods. Motility activity (MA), was calculated as: $M A=M I *$ number of MMC's $* h^{-1}$. The MMC cycle was defined as the time from the end of phase III of the complex to the end of next phase III as identified by the criteria of Code and Marlett (5). Phase III of the MMC was identified as distally propagating regular contractions preceded by phase $\mathrm{Il}$; a period of irregular contractions and followed by phase I; a period of absent contractions. The distinct phases of the MMC were determined visually. The migration velocity of the MMC's was determined semi- 
automatically by assessing the time of passage of the end of phase III along at least 2 channels. The mean migration velocity of the MMC was calculated in $\mathrm{mm}$ per minute.

\section{Statistical analysis}

The data were analyzed by 2-way ANOVA for differences between treatments and differences in time, using as within factor time with 2 levels (Day 0 and 2) and as between-factors treatment with 3 levels (Con, ET-Con and ET-Arg). $P_{T}$ indicates the error alpha values of time effect, $P_{T \times G}$ the interaction of time and treatment group (time $x$ group) and $P_{G}$ the effect of L-arginine treatment. Levels of significance were set at $P<0.05$. Dunnett post-hoc testing was performed for muitiple group comparisons at Day 0 (motility parameters) or Day 1 (NO synthesis rates and arterial arginine concentrations) and Day 2.

\section{Results}

At Day 1, arterial arginine plasma concentrations had decreased in response to the endotoxin infusion period in the ET-Con pigs compared to Con pigs (Figure 3A), indicating that plasma arginine levels are sensitive to endotoxin infusion. At Day 2 that is one day after endotoxin treatment and during enteral feeding, plasma levels of arginine remained decreased. Both intravenous L-arginine infusion and L-arginine administered via the intragastric route increased the arginine concentrations in arterial plasma versus controls, on Day 1 and 2, respectively (Figure 3A). The total production of L-arginine by the splanchnic area between the intragastric L-arginine-supplemented and control group differed about $3.4 \mu \mathrm{mol} \cdot \mathrm{kg} \mathrm{bw}^{-1} \cdot \mathrm{min}^{-1}$ (data not shown) indicating a $65 \%$ recovery of the intragastricly infused L-arginine in the circulation. The rate of NO synthesized by the portal-drained viscera, as measured by $\mathrm{N}_{2}$-arginine to $\mathrm{N}_{1}$-citrulline conversion, was stimulated more than 3 -fold by endotoxin infusion at Day 1 (Figure 3B). Intravenously infused L-arginine on Day 1 was capable of further increasing the portal-drained viscera NO production almost 3-fold (Figure 3B). The synthesis rate of NO by the portal-drained viscera was not significantly increased versus controls on the day after recovery from endotoxemia on Day 2. Intragastrically infused L-arginine on Day 2 was able to significantly increase visceral NO synthesis when compared with endotoxemic controls. 


\section{A: Arginine concentration}

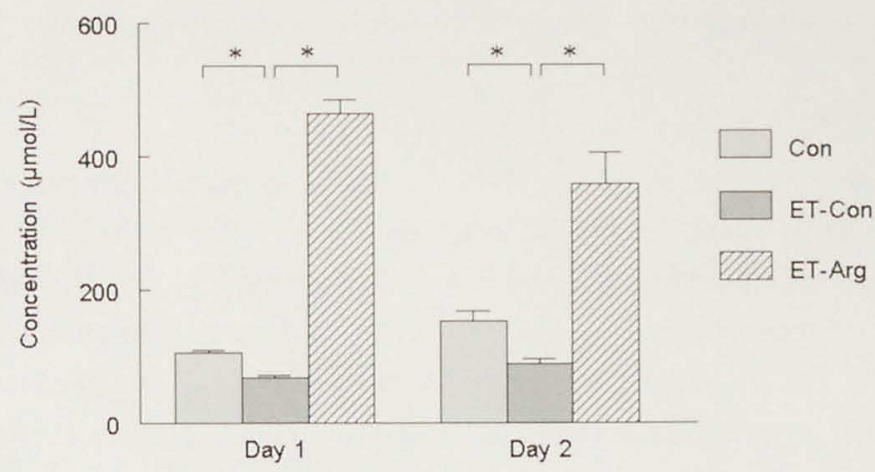

B: Portal-drained viscera NO synthesis

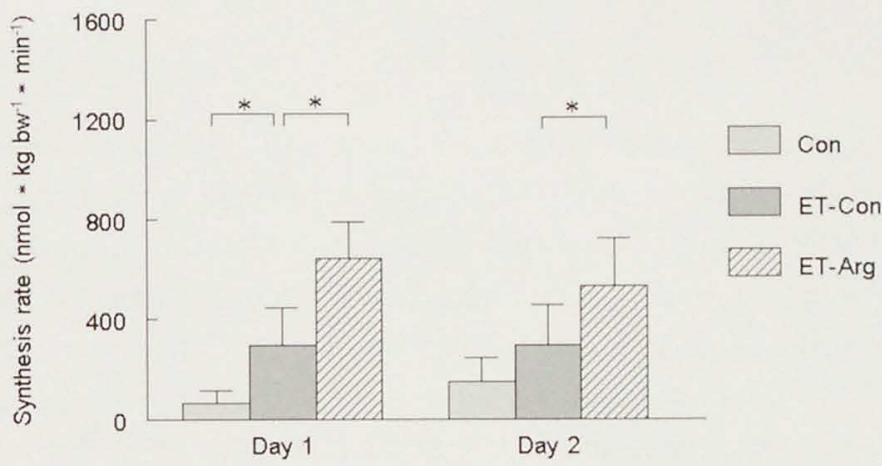

Figure 3. A: Arterial arginine concentrations and B: portal-drained viscera NO production measured from $\mathrm{N}_{2}-$ arginine to $\mathrm{N}_{1}$-citrulline stable isotope conversion at Day 1: during fasting and at Day 2: 1 day after cessation of endotoxin or saline infusion during enteral feeding. Con: receiving saline $(n=6)$, ET-Con: receiving endotoxin + L-alanine $(n=6)$; ET-Arg: receiving endotoxin $+L$-arginine $(n=6)$. Data are means \pm SEM. Statistics by 2 -way ANOVA; $A$ : arginine concentration showed a significant group effect $\left(P_{G}=0.00\right)$ and group $x$ time effect $\left(P_{G X T}=0.006\right)$ while no time effect was observed. B: NO production showed a significant group $x$ time effect $\left(P_{G \times T}=0.04\right)$ while no group or time effect was observed. ${ }^{\star} P<0.05$ by Dunnett post-hoc testing; significant difference between the groups at Day 1 or 2 .

All 12 endotoxin-treated pigs supported the amino acid infusions and no disruption of the MMC pattern was observed, neither during fasting, nor during feeding. As can be observed from Figure 4A, the MMC cycling frequency during fasting and feeding in the Con group was comparable. Endotoxin infusion in the ET-Con pigs resulted in shortening of MMC duration (from $59 \pm 8$ to $34 \pm 1 \mathrm{~min}$ ) and, therefore, increased MMC frequency compared with Con pigs (Fig 4A). Endotoxin-induced shortened MMC duration could be attributed to a reduction in phase II duration (data not shown). This elevated MMC frequency in the ET-Con group was maintained during the day after endotoxin treatment, also due to reduction of phase II duration. Intravenously infused L-arginine in the ET-Arg pigs prolonged the MMC duration (to $54 \pm 1 \mathrm{~min}$ ) thereby reducing the endotoxin-induced 


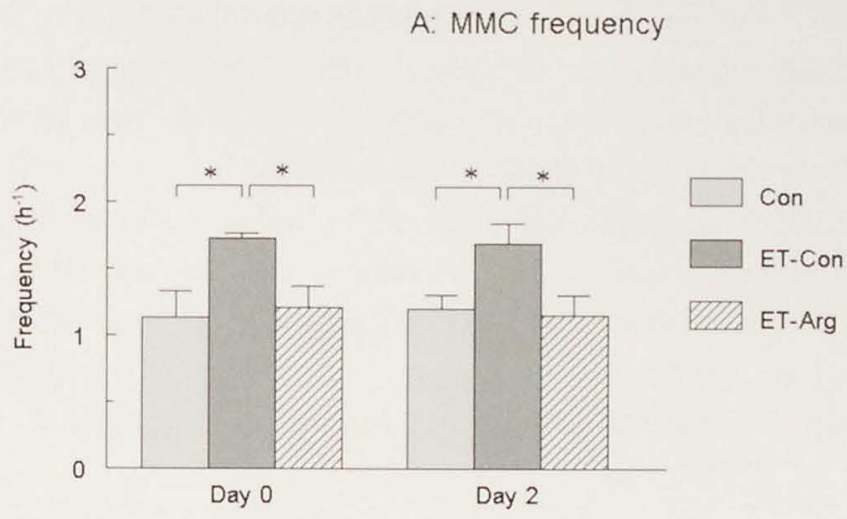

B: MMC migration velocity

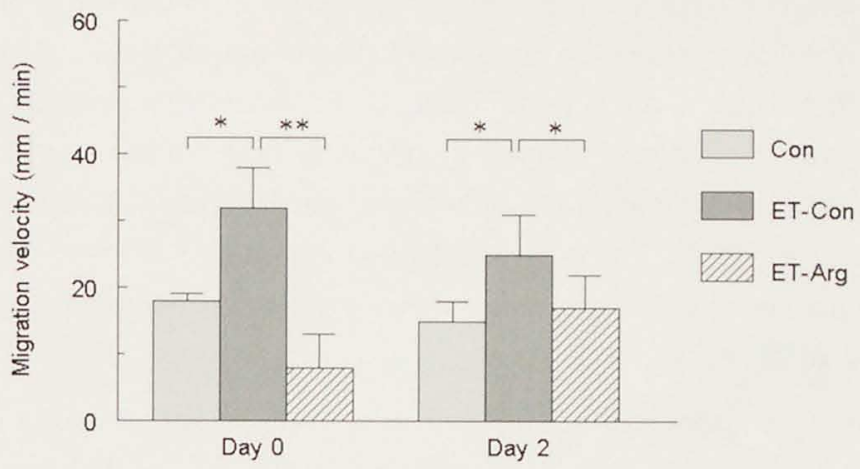

Figure 4. A: Migrating motor complex frequency and B: migrating motor complex migration velocity in the jejunum at Day 0: 24-h recordings during fasting and at Day 2: 8-h recordings 1 day after cessation of endotoxin or saline infusion during enteral feeding. Con: receiving saline $(n=6), E T$-Con: receiving endotoxin $+\mathrm{L}$-alanine $(n=6)$; ET-Arg: receiving endotoxin $+\mathrm{L}$-arginine $(n=6)$. Data are means \pm SEM. Statistics by 2 way ANOVA; $A$ : MMC frequency showed a significant group effect $\left(P_{G}=0.04\right)$ while no group $x$ time or time effect was observed. $B$ : Migrating motor complex velocity showed a significant group effect $\left(P_{G}=0.02\right)$ while no group $x$ time or time effect was observed. ${ }^{\star} \mathrm{P}<0.05$, ${ }^{\star \star} \mathrm{P}<0.01$ by Dunnett post-hoc testing; significant difference between the groups at Day 1 or 2 .

increase in MMC frequency (Figure 4A) to values not significantly different from normal Con values. The $L$-arginine that was intragastricly supplemented to the nutrition from day 1 onward (Figure 4A) also maintained the MMC frequency at a control level although MMC frequency in the ET-Con group remained high. Prolonged MMC duration during arginine infusion was caused by shortening of phase II duration (data not shown).

Endotoxin infusion also resulted in acceleration of MMC phase III propagation along the jejunum on Day 0 from $18 \pm 1$ to $32 \pm 6 \mathrm{~mm} / \mathrm{min}$ (Figure 4B) which was still present two days later (25 \pm 6 in ET-Con vs. $15 \pm 3 \mathrm{~mm} / \mathrm{min}$ in Con). When L-arginine was intravenously administered during endotoxin infusion, the MMC phase III propagation 
was more than 4-fold slower than that of the ET-Con pigs ( $8 \pm 5$ in ET-Arg vs. $32 \pm 6$ $\mathrm{mm} / \mathrm{min}$ in ET-Con) to values not significantly different from Con pigs (Figure $4 B$ ). The Larginine administered intragastricly after endotoxin challenge showed a less pronounced but still significant slowing of MMC migration velocity $(17 \pm 5$ in ET-Arg vs. $25 \pm 6$ $\mathrm{mm} / \mathrm{min}$ in ET-Con) approximating values of the Con pigs. The motility pattern of three and two pigs in the intravenous and intragastric L-alanine treatment group (ET-Con), respectively, was characterized by temporary presence of retrograde MMC's. Also, one intravenously and one intragastricly L-arginine infused pig (ET-Arg) showed retrograde MMC's. Retrograde contractions returned into distally propagating contractions within 1 to 2 hours.

Endotoxin significantly increased the frequency of contractions at Days 0 and 2 (Table 1) compared with controls and increased the amplitude of contractions at Day 2. Intravenous and intragastric L-arginine administration in endotoxin-challenged pigs did not exert a significant effect on the amplitude compared with endotoxemic controls (Table 1). Intravenous L-arginine at Days 0 significantly attenuated the contraction frequency. Whereas endotoxin infusion significantly increased the motility index at Days 0 and 2, simultaneous intravenous L-arginine administration reduced this parameter when compared to the ET-Con pigs, resulting in normalized motility index values (Table 1). Similar effects were achieved by endotoxin and L-arginine intervention on the motility activity values which are shown in Table 1 .

Arginine during 1-day endotoxin infusion normalized the endotoxin-induced increase in mean arterial pressure (Con: $80 \pm 10$, ET-Con: $109 \pm 10$ and ET-Arg: $90 \pm 9 \mathrm{mmHg}$, $\mathrm{P}<0.05 \mathrm{ET}$-Con vs. Con and ET-Arg vs. ET-Con). Although the body temperature was increased by endotoxin infusion on Day $1(39.9 \pm 0.3$ in ET-Con vs. $37.0 \pm 0.4$ in Con, $P<0.05)$, intervention with arginine infusion in endotoxemic pigs had no effect on the increased temperature (39.6 \pm 0.3 in ET-Arg vs. $39.9 \pm 0.3$ in ET-Con, P>0.05). Endotoxin infusion accelerated $(P<0.01)$ the heart rate from $88 \pm 10$ in the Con group to $120 \pm 13$ beats/min in the ET-Con group on Day 1. Intervention with arginine during endotoxin infusion showed a slowing of the heart rate as compared to the ET-Con group (ET-Arg: $104 \pm 14, P<0.05$ ). Although stool quantity was small because of $32-h$ fasting after cessation of intragastric feeding, in both the ET-Con and ET-Arg pigs episodes of watery stool were observed. However, the incidence of diarrhea was higher in the ETCon than in the ET-Arg group ( $83 \%$ vs. $33 \%$, respectively). 
Table 1. Motility pattern of the jejunum of endotoxemic pigs receiving L-arginine (ET-Arg) or L-alanine (ETCon) and non-endotoxemic pigs.

\begin{tabular}{|c|c|c|c|c|c|c|c|}
\hline & & \multicolumn{3}{|c|}{ FASTING } & \multicolumn{3}{|c|}{ FED } \\
\hline & & \multicolumn{3}{|c|}{ Day 0} & \multicolumn{3}{|c|}{ Day 2} \\
\hline & & Con & ET-Con & ET-Arg & Con & ET-Con & ET-Arg \\
\hline \multirow[t]{4}{*}{ Contraction } & Frequency $\left(\min ^{-1}\right)$ & $2.1 \pm 0.8$ & $3.7 \pm 0.5$ & $2.5 \pm 0.5^{8}$ & $2.3 \pm 0.2$ & $3.8 \pm 0.6^{\star}$ & $3.1 \pm 0.4$ \\
\hline & Amplitude $(\mathrm{mmHg})$ & $3.4 \pm 0.3$ & $3.7 \pm 0.2$ & $3.4 \pm 0.3$ & $2.6 \pm 0.2$ & $4.1 \pm 0.4^{*}$ & $3.5 \pm 0.4$ \\
\hline & Motility Index & $10.9 \pm 0.5$ & $12.0 \pm 0.4$ & $11.1 \pm 0.3^{8}$ & $10.8 \pm 0.1$ & $12.3 \pm 0.4^{*}$ & $11.1 \pm 0.3^{8}$ \\
\hline & Motility Activity & $12 \pm 2.4$ & $20.8 \pm 1.3$ & $13.4 \pm 1.8^{8}$ & $13 \pm 1.2$ & $20.3 \pm 1.9^{*}$ & $12.8 \pm 2.1^{8}$ \\
\hline
\end{tabular}

Day 0: 24-h recordings and Day 2: 8 -h recordings. Con: $n=6$, ET-Con: $n=6$ and ET-Arg: $n=6$. Data represent means \pm SEM. Statistics by 2 -way ANOVA; all motility parameters showed a significant group effect $\left(P_{G}<0.05\right)$. The contraction amplitude showed a time $x$ group effect $\left(P_{T \times G}<0.05\right)$. No time effects were observed. Dunnett post-hoc testing at Day 0 or $2 ;{ }^{*} \mathrm{P}<0.05$ significant different vs. Con group and ${ }^{8} \mathrm{P}<0.05$ significant different vs. ET-Con group.Discussion

Endotoxin infection is a main cause of the motility disturbances associated with sepsis (4. 17). In this endotoxemia model we endeavored to reproduce a hyperdynamic state resembling the clinical situation observed in compensated sepsis (11) for which purpose pigs were infused small doses' of endotoxin simultaneously with fluid supportive therapy over a 24-h period. The hemodynamic response of the pigs to the endotoxin infusion, which included preserved blood flow to the portal-drained viscera and elevated heart rate and mean arterial pressure, represents the development of a state characteristic of compensated sepsis. Moreover, hyperdynamic endotoxemia was characterized by increased MMC cycling frequency and accelerated MMC migration velocity along the jejunum (unpublished results, Bruins et al, 2001).

Nitric oxide synthesized by NOS-I in the nonadrenergic noncholinergic (NANC) nerves of the small intestine has been attributed a main mediator function in motor activity (20). Based on the hypothesis that NOS-1 mediated NO production is essential for sustenance of MMC cycling and that plasma levels of L-arginine become limiting for basal NO production during prolonged endotoxemia, endotoxemic pigs were treated with Larginine. The results of this study demonstrate that exogenous L-arginine supplementation exerts an inhibitory effect on the intestinal hypermotility pattern that is observed during hyperdynamic endotoxemia and at one day after endotoxemia, resulting in a normalization of the motility pattern.

In this model of sepsis, endotoxin infusion did not interrupt the regular MMC cycling along the jejunum neither in L-arginine nor in L-alanine treated pigs. The regular periodicity of MMC cycling characteristic of the fasting state was maintained during the continuous enteral feeding in both controls and endotoxin challenged pigs. It therefore appears that low rates of constantly infused endotoxin, as well as, enteral nutrition maintain the repetitive pattern of MMC's in the porcine jejunum. A partial recovery of the intragastricly infused arginine explains the (not significantly) lower arginine plasma 
concentration in the intragastricly arginine-treated pigs on Day 2 as compared to intravenously arginine-treated pigs on Day 1 . The increased MMC cycling frequency and MMC propagation velocity along the jejunum during prolonged endotoxin infusion and at one day postendotoxin may cause accelerated propulsive activity, resulting in rapid transit of jejunal contents. Increased intestinal transit was reported in rats that were administered E. coli endotoxin (9). Rapid transit along the gastrointestinal tract is also a common feature in human subjects suffering from diarrhea caused by enterotoxemic $E$. coli infection (6). The rapid jejunal MMC cycling velocity therefore may have contributed to the watery diarrhea that was observed in the endotoxemic control pigs. This is confirmed by the lower incidence of diarrhea in the arginine-treated endotoxemic pigs in which the MMC pattern had normalized. The increased motility indexes that were manifest during and after endotoxemia do not correspond with the decreased motility indexes that were reported in the jejunum of dogs treated with a single bolus of endotoxin (7). Discrepancies in observations may rely upon the way of endotoxin administration, i.e., a single bolus versus continuous treatment with endotoxin but also on a dose-dependent response to chronic endotoxin infusion as has been observed for jejunal transit data (8). Intravenous L-arginine supplementation during acute endotoxemia reduced endotoxin-induced increased MMC frequency and slowed the endotoxininduced acceleration of MMC propagation along the jejunum to normal values. Two days after endotoxin treatment, a comparable effect was achieved by intragastric L-arginine supplementation of the enteral diet. The increased jejunal motility index which developed upon endotoxin treatment was reversed by intravenous as well as one day after endotoxin by intragastric L-arginine supplementation. These observations show that both intravenous and intragastric L-arginine supplementation exerted a normalization of the MMC characteristics under (post)endotoxemic circumstances.

Endotoxin infusion significantly increased the conversion of ${ }^{15} \mathrm{~N}_{2}$-arginine to ${ }^{15} \mathrm{~N}$-citrulline across the portai-drained viscera, indicating stimulation of visceral NO production. The chronically infused endotoxin may predominantly account for the increase in NO synthesis via induced NOS-II activity. One day after endotoxin, NO synthesis by the portal-drained viscera was no longer significantly increased. These results obtained nevertheless do not allow us to distinguish between NO derived from NO produced by constitutive NOS (NOS-I and -III), especially neuronal NOS (NOS-I) associated with NANC innervation and NO produced by inducible NOS (NOS-11), which is predominantly expressed in muscularis macrophages after immune stimulation (10). During and after endotoxemia circulating levels of L-arginine were reduced. When extracellular supply of L-arginine is limited, NOS-II can compete with constitutive NOS for their common substrate and thus limit the L-arginine availability for constitutive NOS-mediated NO synthesis in the gut. Moreover, constitutive NOS-mediated NO synthesis may become 
compromised, since endotoxin administration was shown to inhibit constitutive NOS activity concomitant with increased NOS-II activity (1). Both intravenously infused Larginine in parallel with endotoxemia and L-arginine administered by the intragastric route one day postendotoxemia were able to further increase the NO production by the portal-drained viscera. We speculated that L-arginine supplementation during prolonged endotoxemia mediated its effect through overcoming impaired NO synthesis by the constitutive NOS isoforms, which is delineated by its capacity to evoke an additional increase in visceral NO production. These observations suggest that L-arginine concentration, and thus, NO substrate availability under endotoxemic conditions determines the synthesis rate of NO by the portal-drained viscera.

In this study, L-arginine supplementation normalized the endotoxin-induced disturbance in the motility pattern by reducing the increased $M M C$ cycling frequency and decelerating the rapid MMC migratory velocity. The inhibitory effect of L-arginine on the MMC cycle length and contraction frequency that we observed under endotoxemic conditions seems consistent with findings in dogs, although here it was achieved under normal physiological circumstances (15). Moreover, experiments in healthy humans using the NOS inhibitor L-NG-monomethyl-arginine (L-NMMA) showed a reduction of the MMC cycle length (20) implicating an inhibitory role for NO in the intestinal motor activity. It, therefore, appears that the neuronal NO produced by NOS-I plays a major role in MMC cycling by suppressing the MMC cycling frequency, MMC velocity and motility index. $A$ restraint in neuronal NO-mediated nitrergic inhibition of motility activity would explain the increased jejunal motility activity present during endotoxemia. Moreover, L-arginine supplementation could be involved in the normalization of the MMC cycling pattern and migration velocity, and possibly the motiiity index, through restoration of compromised NO synthesis by the nitrergic NANC nerves in the visceral organs.

In conclusion, exogenous L-arginine exerted an inhibitory effect on jejunal motor activity during endotoxemia and, thereby, restored the rapid $M M C$ cycling and propulsion during endotoxin to normal values. It can be speculated that basal levels of L-arginine are required for sustenance of NO synthesis by the NANC nerves that are involved in the control of regular motor activity. Although further investigation is required, it can be speculated that L-arginine supplementation may be beneficial under conditions of acute and long-term septicemia by overcoming reduced plasma L-arginine levels, and therefore reduced neuronal NO synthesis.

\section{Acknowledgements}

This study was supported by the Netherlands Organization for Scientific Research (project 902-23098). The authors acknowledge Mrs. G.A.M. ten Have for experimental help. 


\section{References}

1. Beach, P. K., D. A. Spain, T. Kawabe, P. D. Harris, and R. N. Garrison. Sepsis increases NOS-2 activity and decreases non-NOSmediated acetylcholine-induced dilation in rat aorta. J Surg Res 96: 17-22., 2001.

2. Bruins, M. J., P. B. Soeters, and N. E. Deutz. Endotoxemia affects organ protein metabolism differently during prolonged feeding in pigs. $J$ Nutr 130: 3003-13., 2000.

3. Calignano, A., B. J. R. Whittle, M. DiRosa, and S. Moncada. Involvement of endogenous nitric oxide in the regulation of rat intestinal motility in vivo. Eur J Pharmacol 37: 273-276, 1992.

4. Closs, E. I., F. Enseleit, D. Koesling, J. M. Pfeilschifter, P. M. Schwarz, and U. Forstermann. Coexpression of inducible NO synthase and soluble guanylyl cyclase in colonic enterocytes: a pathophysiologic signaling pathway for the initiation of diarrhea by gram-negative bacteria? Faseb $J 12$ : 1643-9, 1998.

5. Code, C. F., and J. A. Marlett. The interdigestive myo-electric complex of the stomach and small bowel of dogs. I Physiol (Lond) 246: 289-309, 1975.

6. Cohen, M. B., and R. A. Giannella. Enterotoxigenic Escherichia coli. New York: Raven Press, Ltd., 1995.

7. Cullen, J. J., D. K. Caropreso, K. S. Ephgrave, L. L. Hemann, and M. M. Hinkhouse. The effect of endotoxin on canine jejunal motility and transit. J Surg Res 67: 54-7, 1997.

8. Cullen, J. J., R. C. Doty, E. K.S., H. M.M., and K. Broadhurst. Changes in intestinal transit and absorption during endotoxemia are dose dependent. J Surg Res 81: 81-86, 1999.

9. Cullen, J. J., D. Mercer, M. Hinkhouse, K. S. Ephgrave, and J. L. Conklin. Effects of endotoxin on regulation of intestinal smooth muscle nitric oxide synthase and intestinal transit. Surgery 125: 339-44, 1999.

10. Eskandari, M. K., J. C. Kalff, T. R. Billiar, K. K. Lee, and A. J. Bauer. LPS-induced muscularis macrophage nitric oxide suppresses rat jejunal circular muscle activity. Am J Physiol 277: G478-86, 1999.

11. Fink, M. P.. and S. O. Heard. Laboratory models of sepsis and septic shock. $J$ Surg Res 49: 186-196, 1990.
12. Freund, H., S. Atamian, J. Holroyde, and J. E. Fischer. Plasma amino acids as predictors of the severity and outcome of sepsis. Ann Surg 190: $571-6,1979$.

13. Glasgow, 1., K. Mattar, and A. Krantis. Rat gastroduodenal motility in vivo: involvement of NO and ATP in spontaneous motor activity. Am J Physiol 275: G889-96., 1998.

14. Lefebvre, R. A., and L. Bartho. Mechanism of nitric oxide-induced contraction in the rat isolated small intestine. $\mathrm{Br} J$ Pharmacol 120: 975-981, 1997.

15. Maczka, M., P. Thor, K. Lorens, and S. J. Konturek. Nitric oxide inhibits the myoelectric activity of the small intestine in dogs. J Physiol Pharmacol 44: 31-42, 1993.

16. Meyer, W. Clinical aspects, pathogenesis and therapy of shock due to sepsis caused by gramnegative organisms. Schweiz Med Wochenschr 96: $137-43,1966$.

17. Moon, H. W. Pathogenesis of enteric diseases caused by Escherichia coli. Adv Vet Sci Comp Med 18: 179-211, 1974.

18. Powell, D. W. Approach to the Patient with Diarrhea. In: Textbook of Gastroenterology, edited by $T$. Yamada. Philadelphia: JB Lippincott Company, 1995, p. 813-840.

19. Rodriguez-Membrilla, A., V. Martinez, $M$. Jiminez, E. Goñalons, and P. Vegara. Is nitric oxide the final mediator regulating the migrating myoelectric complex cycle? Gastrointest Liver Physiol 268: G207-G214, 1995.

20. Russo, A., R. Fraser, K. Adachi, M. Horowitz, and $G$. Boeckxstaens. Evidence that nitric oxide mechanisms regulate small intestinal motility in humans. Gut 44: 72-6, 1999.

21. Salzman, A. L., and L. Andrew. Nitric oxide in the gut. New Horizons 3: 352-364, 1995.

22. Sarna, S. K., M. F. Otterson, R. P. Ryan, and V. E. Cowles. Nitric oxide regulates migrating motor complex cycling and its postprandial disruption. Am $J$ Physiol 265: G759-G766, 1993.

23. Thompson, J. S. The intestinal response to critical illness. Am J Gastroenterol 90: 190-200, 1995.

24. Wirthlin, D. J., J. J. Cullen, S. T. Spates, J. L. Conklin, J. Murray, D. K. Caropreso, and K. S. Ephgrave. Gastrointestinal transit during endotoxemia: the role of nitric oxide. J Surg Res 60: 307-11, 1996. 
CHAPTER 11

GENERAL DISCUSSION

Maaike J Bruins

Nicolaas EP Deutz

Peter B Soeters

Department of Surgery, Maastricht University, The Netherlands 


\section{General Discussion}

Nitric oxide (NO) is synthesized enzymatically from the amino acid L-arginine by the NO synthase (NOS) enzyme. Whereas the neuronal NOS (NOS-I) and endothelial NOS (NOS-III) isoforms are expressed constitutively, expression of the inducible NOS isoform (NOS-II) requires induction under the influence of endotoxin or various cytokines. The role of NO in sepsis is controversial. Under normal physiological circumstances, NO synthesized in low rates by the constitutive NOS isoforms acts as a mediator molecule in the immune response (a), in blood pressure regulation and blood flow distribution (b) and in neuronal communications (c).

High synthesis rates of NO resulting from NOS-II induction initially have been implicated as being an important factor contributing to the hemodynamic and metabolic disturbances observed in septic shock (26). On the other hand, increasing evidence accumulates that L-arginine as precursor of NO may exert beneficial effects under stress conditions such as sepsis, trauma and post-operative conditions. Elevated L-arginine utilization and impaired L-arginine de novo synthesis are potential contributors to the depletion of circulating L-arginine often observed under conditions of sustained sepsis. Supplementation of endotoxemic rats and septic patients with L-arginine was associated with improved immune status and enhanced nitrogen balance (19). However, because of the addition of multiple supplements to these enteral formulas, the beneficial effect could not uniquely be attributed to L-arginine.

First, we tried to obtain insight in essential alterations manifest during endotoxin-induced sepsis and recovery thereof. For that purpose, a hyperdynamic model of sepsis was designed to assess endotoxin-induced changes at organ and whole body level.

The experimental set-up aimed to induce an experimental sub-acute hypermetabolic model of sepsis that would resemble the hyperdynamic septic state that is encountered in septic patients receiving modern intensive care. For that purpose, pigs were challenged with endotoxin of the Escherichia coli subtype and co-infused with isotonic saline. Administration of small doses of endotoxin over an extended period resembles the continuous release of endotoxins into the circulation, which is thought to occur in most patients with sepsis. This, therefore, better resembles human sepsis. The endotoxemic pigs were supported by generous fluid infusion to compensate for hypovolemia in order to preserve cardiac output and organ perfusion eliciting a hyperdynamic response representative for hypermetabolic human sepsis (20). This experimental model of sepsis allows studies over several days because the model induces a very low mortality rate. 
The decision to initiate artificial nutrition in hospitalized patients is based on the presence of starvation of long duration. Artificial nutrition has been claimed to lower the incidence of organ failure and to have a survival advantage (18) and has been demonstrated to diminish septic morbidity. Initially, nutritional support is often at least in part delivered via the intravenous infusion route whereas enteral nutrition should be instituted as soon as possible. To mimic the clinical situation, the endotoxemic insult was followed by enteral feeding to assess the prolonged effects of endotoxin in a model of nutrition-supported post-septicemia. Long-term effect of endotoxin was assessed 1 and 4 days postendotoxemia during enteral feeding of a liquid protein-enriched meal. The effect of endotoxin challenge was studied by comparing pigs treated with endotoxin with their mock-treated controls that underwent the same treatments except for the endotoxin infusion. Therefore, the main aims of the study consisted of the study of the immediate and prolonged effect of endotoxin challenge on fasting endotoxemic pigs and nourished post-endotoxemic pigs, respectively.

The acute effects of endotoxin were assessed 24-h after the start of endotoxin infusion in the fasting state. Insight in the endotoxin-induced basic alterations in the L-arginine-NO metabolism is required before changing NO production rates by means of L-arginine supplementation. Negative nitrogen balance associated with progressive loss of body cell mass, altered glucose metabolism and impaired motility function, constitute the hallmark of the response to prolonged stress such as sepsis (10). To validate the endotoxin-induced sepsis model, we focused on changes in amino acid fluxes and protein synthesis and degradation in the different organs and inter-organ amino acid fluxes. Also, alterations in organ glucose and lactate metabolism and jejunal motility parameters were studied during and after induction of endotoxemia. In addition, the effect of endotoxin infusion on the different hemodynamic parameters was followed in time to validate the hyperdynamic sepsis model.

In the experiments described in this thesis, the impact of L-arginine supplementation on endotoxin-induced changes was assessed under endotoxemicnd post-endotoxemic conditions. Exogenous L-arginine was supplied either via the intravenous route during endotoxemia, or via the intragastric route during post-endotoxemia. The NO modulating capacity of L-arginine administration during and after endotoxemia was studied by determining the changes in organ and whole-body arginine-to-citrulline isotope conversion. Further, the effect of L-arginine intervention on the different aspects of amino acid and protein metabolism, hemodynamic parameters and jejunal motility was evaluated. 


\section{Protein and glucose metabolism}

\subsection{Fasting}

In the first experiment endotoxin was infused over a 24-h fasting period. Due to the experimental setting we were able to differentiate between alterations caused by $24-\mathrm{h}$ fasting and by endotoxin infusion as such. The data (chapter 3 ) indicate that after a period of 24-h fasting the rate of net protein breakdown in the hindquarter and portaldrained viscera during the period is reduced while protein synthesis and degradation (i.e., turnover) in the liver was also reduced. Decreased organ protein breakdown was, nevertheless, not detectable on a whole body level. These observations are consistent with earlier findings in the rat showing diminished protein synthesis and net protein loss in the phase between short and long-term starvation $(15,16)$. Reduced organ protein turnover has been proposed to constitute a protein conserving mechanism (38).

\subsection{Endotoxin}

Sepsis leads to severe modifications in organ protein metabolism resulting in net protein catabolism. Skeletal muscle protein wasting is a prominent feature of the metabolic response to sepsis. Knowledge concerning the effect of sepsis on protein turnover of other organs is relatively poor. Persistent protein wasting leads to muscle dysfunction and prolongs recovery from the septic insult. Endotoxin-induced release of inflammatory cytokines, hormones and their interactions are assumed to play an important role in mediating net protein catabolism through modulation of the protein synthesis and degradation enzymes and inhibition of amino acid transport (22). In addition, visceral (30) and muscle (35) protein is depleted under conditions of prolonged starvation and accretion is achieved when amino acids are provided (3), suggesting that protein synthesis is dependent on the amino acid availability under conditions of increased requirement.

\section{Hindquarter}

After 24-h of endotoxin infusion acceleration in protein breakdown of the skeletal muscle (calculated as $2 \times$ hindquarter area) was observed whereas protein synthesis was not significantly changed (chapter 3 ). Enhanced muscle proteolysis constitutes one of the most striking features in sepsis. Moreover, in literature, some sepsis studies suggest inhibition of protein synthesis also to contribute to net muscle protein catabolism. This, however, may not be the case in our model of "mild" endotoxemia. Increased protein degradation results in substrate supply for energy consuming metabolic processes in liver, other visceral organs, immune cells and the muscle itself. At the whole body level, the hindquarter predominantly accounted for the provision of substrates including lactate, arginine, glutamate, glutamine, leucine and alanine, wholly or in part resulting from accelerated protein degradation. Pyruvate, 
generated in muscle, can be transaminated to alanine, which is returned to the liver for gluconeogenesis. This process may additionally contribute to the enhanced alanine efflux. This transamination reaction requires an $\alpha$-amino acid as donor of the amino group, generating $\alpha$-keto acid in this process. In view of the fact that the latter is a precursor of glutamate, an increased transamination rate appears to contribute to the observed increased glutamate efflux. Glutamate also provides substrate for glutamine synthesis by glutamine synthetase, which is increased under endotoxemic conditions. BCAA's serve as main amino group donor in the transamination process although BCAA production from protein degradation seems to exceed BCAA utilization since endotoxin turned net BCAA influx into efflux.

Increased muscle protein degradation during endotoxin infusion (chapter 3) was normalized one day post-endotoxemia (after cessation of endotoxin infusion when protein-enriched enteral nutrition was administered) (chapter 4). Four days postendotoxemia (chapter 4), both hindquarter protein synthesis and degradation (i.e., protein turnover) were enhanced resulting in net protein gain. This increased protein turnover most likely represents recovery of endotoxin-induced wasted muscle proteins. Moreover, enhanced glutamine efflux and reduced alanine uptake that was observed 1 and 4 days after endotoxemia implies that synthesis of these amino acids is sustained.

During endotoxemia, hindquarter consumption of glucose increased simultaneously with lactate release from the hindquarter reflecting increased glycolysis in muscle (25) (chapter 3). Pyruvate is increasingly formed by glycolysis and, subsequently, is converted to lactate which was reflected in elevated lactate efflux. Enhanced hindquarter glucose consumption and lactate release were abolished after the endotoxin infusion period during feeding (chapter 4).

Liver The main feature of sepsis is the increased transfer of substrate from the periphery to the liver. The liver acted during endotoxemia as a major consumer of amino acids, non-essential gluconeogenic amino acids (including glutamine, glycine and alanine) and essential gluconeogenic amino acids (including the BCAA, tyrosine, and phenylalanine). The liver plays a central role in de novo formation of glucose, but also, of proteins. The cycle in which transaminated alanine from muscle is transported to the liver for production of glucose which is, subsequently, returned to the muscle, is termed the glucose-alanine cycle or Felig cycle. Lactate, which is transported from the muscle to the liver, can be converted to glucose and, then, returned to the muscle to replenish energy stores. This cycle is termed the Cori cycle. Derangements in skeletal muscle glucose metabolism evoked by sepsis were thought to determine the rate of gluconeogenesis in the liver whereas, in contrast, accelerated rates of gluconeogenesis do not drive net catabolism in skeletal muscle (47). Increased nitrogen supply from amino acid 
degradation in gluconeogenesis by the liver involves enhanced liver urea cycling and ammonia uptake although urea efflux was not significantly increased in the endotoxemic pigs. Enhanced ureagenesis is a means to clear nitrogenous waste from elevated amino acid supply. During sepsis, the increase in hepatic glucose production stoichiometrically matched the increase in glucose utilization by the portal-drained viscera and muscle $(2 \times$ hindquarter). Besides hepatic gluconeogenesis, renal gluconeogenesis also contributes to whole body glucose production. The liver consumed more lactate than released from the portal-drained viscera and muscle together, implying that other organs, possibly the brain and erythrocytes are involved in lactate production.

Increased consumption of amino acids by the liver may not only serve augmented gluconeogenesis, but also protein synthesis. Accordingly (chapter 3), increased liver protein synthesis was observed as result of endotoxin infusion with no change in protein degradation. Under septic conditions, the liver synthesizes proteins, the so-called acute phase proteins (48) that are involved in the host-defense response. Enhanced net protein synthesis in the liver was still observed 1 and even 4 days after endotoxin infusion during enteral feeding (chapter 4). The net protein synthesis that was manifest during short-term and long-term recovery from endotoxemia resulted from both increased protein synthesis and decreased protein degradation. It is in fact known that the acute phase response can last for several days (6). Liver glucose and urea output were no longer increased one day after endotoxin treatment (chapter 4). Therefore, the increased amino acid consumption by the liver predominantly serves protein synthesis, which appears enhanced in the postendotoxemic condition (chapter 4). The higher hepatic glutamate and lower urea release into the circulation one day post-endotoxemia (both not significant) may constitute a major mechanism for nitrogen salvage since glutamate can serve as nitrogen source for extrasplanchnic tissues (40).

Portal-drained viscera

Protein synthesis and degradation of the portaldrained viscera remained unaffected during endotoxemia (chapter 3). These observations contrast with rat studies that demonstrated increased gut protein synthesis under endotoxemic conditions (23). Discrepancies may be explained by differences in experimental endotoxin model. Net glucose release and lactate consumption across the portal-drained viscera concomitantly increased during endotoxin infusion, reflecting elevated glycolytic activity. Since protein synthesis in the portal-drained viscera remained unchanged, increased net BCAA consumption presumably reflects increased BCAA transamination in the smooth muscle of the gastrointestinal tract during endotoxemia. Portal-drained viscera influx of glutamine reduced simultaneous with efflux of arginine, leucine and alanine. Nevertheless, an increase in total amino acid efflux was observed which was predominantly due to increased glycine and tyrosine effluxes. One and 4 days after endotoxemia in the fed condition, protein synthesis and degradation in the portal- 
drained viscera were still unaffected. Also, glucose and lactate fluxes were unaltered. A marked increase in net release of all amino acids from the portal-drained viscera was observed except for glutamate, net release of which significantly decreased. Net glutamine uptake by the portal-drained viscera was completely abolished in the postendotoxemic pigs. Since protein degradation was not increased 1 and 4 days postendotoxemia, this may suggest that the gut is capable of de novo amino acid synthesis from ammonia and glutamate as is known to occur by means of enteric flora in the gut wall (32). This would subsequently lead to increased amino acid appearance in the portal vein. Therefore, in the post-endotoxemic state, not only elevated amino acid release from muscle but also from portal-drained viscera substantially contribute to amino acid supply to the liver for enhanced protein synthesis.

Trauma, sepsis, and other conditions of stress are characterized by a hypermetabolic state. Characteristics of altered glucose metabolism constitute accelerated gluconeogenesis, glucose oxidation and utilization. Changes in protein metabolism include increased protein breakdown, amino acid oxidation and urea synthesis. Peripheral proteolysis, transamination and glycolysis lead to fluxes of carbon and nitrogen that support metabolic processes (energy provision, gluconeogenesis, acute phase protein synthesis) in immune cells and central organs involved in host defense.

In the hypermetabolic state, increased substrate availability is required to meet energy demands for tissue repair and host defenses. Sustained hypermetabolism in progressive septic patients not only leads to muscle depletion and catabolism, but also to the inability of the patient to meet these increased metabolic demands subsequently resulting in accelerated visceral protein depletion, impaired immune function and wound healing.

The time and the amount of protein and calories necessary for optimal nutritional support during acute phases of illness remains a subject of debate. Additional studies regarding the metabolic effects of enteral feeding under conditions of septicemia are required to devise a strategy for the nutritional treatment of critically ill patients. Nutritional support in critically ill patients often except for citrulline and ornithine preventing the mobilisation of endogenous glucose, fat and protein stores and the breakdown of lean tissue continues (10). This may be due to the hormonal alterations and cytokine cascade programming peripheral tissues to be catabolic, generating substrate for the central organs to be anabolic (liver, other immune system, wound) that are involved in the metabolic perturbations accompanying catabolic sepsis $(13,21)$. Nevertheless, adequate provision of nutritional substrates may meet the increased organ substrate demands in the presence of a severe inflammatory stimulus. Supporting metabolic processes rather than preventing proteolysis, glycolysis and lipolysis should be the goal of early enteral feeding of critically ill patients (4). 
The proposition that the net protein gain achieved by amino acid supply is caused by reduced protein degradation (43) is out of date. Under healthy conditions, the increase in muscle protein synthesis after feeding is mediated by the rise in plasma insulin (35). Also in injured rats, glucose and amino acid supply favored protein synthesis (36). Enteral supply of nutrition in our healthy pigs not only showed capable of increasing organ protein synthesis, but protein breakdown as well although to a much lesser extent, resulting in net protein gain (data not shown). Simultaneous stimulation of protein synthesis and breakdown by amino acids has also been proposed by Campbell et al (10). Therefore, nutritional intervention in the recovery phase of sepsis facilitates the replenishment of depleted energy stores and restores wasted proteins when insulin resistance has subsided.

With respect to protein synthesis, the liver, the portal-drained viscera and hindquarter all showed a protracted response to endotoxin challenge (chapter 4). Increased amino acid release by the portal-drained viscera was manifest 1 day after endotoxemia and lasted for at least 4 days after endotoxemia whereas protein turnover remained unchanged. This possibly reflects synthesis of non-essential amino acids by the microflora from glutamate (32), thereby, contributing to gluconeogenic precursor supply when protein intake is low. Muscle showed a pronounced difference between early phase protein catabolism after 1 day of endotoxin infusion (chapter 3 ) and late phase protein anabolism 4 days after endotoxin infusion. The net muscle protein gain that was manifest in the late phase during feeding was due to enhanced protein synthesis exceeding accelerated protein degradation when compared to control pigs. Also, 1 and 4 days after endotoxemia, disposal and production of arginine and valine by the muscle were increased, the first exceeding the latter, resulting in net uptake of these amino acids. It is unclear how enteral nutrition is involved in this protracted response. These observations agree with the idea that nutritional support under septic conditions is not capable to arrest protein breakdown.

The observed increase of liver protein synthesis during endotoxin infusion was sustained 4 days after abolition of the endotoxin challenge. Like in muscle, increased substrate availability from nutrition may sustain this enhanced liver protein synthesis in the recovery phase of sepsis. In fact, amino acid infusions in injured rats increased muscle protein synthesis and further increased the liver protein synthesis as compared to fasting rats (36). In the pigs recovering from endotoxin challenge, increased hepatic and whole body glucose production was blunted. It is unclear whether the nutritional intervention was instrumental in this. Nevertheless, in other studies enteral carbohydrate administration in critically ill patients failed to suppress endogenous glucose production and to promote net splanchnic glucose uptake (45). 
Our endotoxemia model does not allow elucidating whether early enteral nutrition is of assistance in sustaining metabolic processes such as protein synthesis, thereby, more rapidly compensating the catabolic response to sepsis. For that purpose, in the future a post-endotoxemic group deprived of enteral nutrition would be of interest. High protein turnover has been put forward to facilitate elimination of damaged proteins (38). Considering that disturbed glucose and protein breakdown in response to injury are difficult to reduce with current nutritional therapy (11), nutritional support in principle should be designed to sustain substrate requirements necessary for adequate host response, largely taking place in liver, immune system and wound, and not in muscle tissue.

\subsection{Arginine intervention}

When L-arginine was applied as therapeutic intervention during the endotoxemia period, whole body protein breakdown and synthesis were reduced (chapter 5). An inhibition in liver protein turnover exceeding an increase in hindquarter protein turnover accounted for this reduction. Portal-drained viscera and renal protein turnover remained unaffected by L-arginine supplementation. Enhanced hindquarter protein turnover and decreased liver protein turnover were still present one day after endotoxemia when L-arginine was supplemented in the enteral nutrition (chapter 5). The latter may imply that L-arginine down-regulates the severity of the hepatic stress response to tissue injury and inflammation.

\section{Arginine and NO Metabolism}

\subsection{Fasting}

During a 24-h fasting period, the whole body appearance rate of L-arginine diminished. The decrease in muscle protein degradation accounted for this as exemplified by the protein kinetics in the hindquarter (chapter 3).

\subsection{Endotoxin}

When pigs were challenged with endotoxin, increased hindquarter protein breakdown resulted in increased whole body appearance of arginine (chapter 3 ). The decrease in whole body arginine appearance caused by 24-h fasting was of the same magnitude as the increase caused by 24-h endotoxin (chapter 6 ). This implies that under conditions of reduced arginine availability such as fasting, muscle to become an important source of arginine due to presence of endotoxin. Although whole body arginine appearance rate was higher, plasma arginine levels were in endotoxin treated pigs compared to controls suggesting that arginine utilization exceeds arginine production. Plasma arginine reached half-saturating levels, potentially indicating that extracellular arginine availability 
for induced NO synthesis by NOS-II may become limiting $(39,42)$. The arginine-tocitrulline isotope conversion technique provided evidence that endotoxin stimulated the portal-drained viscera and liver NO synthesis (chapter 6) despite rate-limiting arginine levels. The elevated NO production by the portai-drained viscera and liver occurred simultaneously with increased arginine disposal by these organs. Endotoxin-induced NOS-II enzyme activity is very likely to be responsible for increased visceral and hepatic NO synthesis.

\subsection{Arginine intervention}

Intravenously infused L-arginine increased the whole body total appearance rate of Larginine 3-fold (chapter 7 and 8 ). The same rate of L-arginine infused via the intragastric route resulted in a 2-fold rise in whole-body arginine appearance, the splanchnic extraction accounting for a proportion of disappearing arginine. L-Arginine infusion simultaneously decreased the arginine de novo synthesis as measured from citrulline-toarginine stable isotope conversion (chapter 8 ). Since increased urea appearance was also observed during L-arginine infusion, it appears that increased arginine degradation together with decreased arginine de novo synthesis regulate the arginine homeostasis under arginine-enriched conditions. Increased arginine appearance promoted NO synthesis in the whole body circulation, which could be accounted for by the portaldrained viscera, the liver, the kidneys and, though less pronounced, the hindquarter (chapter 7). Whole-body, but also organ NO synthesis rate appears strongly related to whole body arginine appearance rate. Enhanced organ NO production paralleled increased arginine disposal. Chapter 8 shows that L-arginine supplementation during and after endotoxemia resulted in increased arginine net uptake by the organs which may represent elevated organ metabolism including accelerated arginine degradation by the arginase and NOS pathways. Plasma ornithine levels were increased in L-arginine infused pigs whereas citrulline levels were reduced. The same was observed for ornithine and citrulline concentrations in jejunal tissue of arginine-treated pigs. Hence, arginine appears the principal source of plasma ornithine, but plasma ornithine not of citrulline. The observed decrease in endogenous arginine appearance rate could partialiy be attributed to the lowered arginine de novo synthesis. Moreover, arginine seems strongly involved in glutamine metabolism. In the liver and kidneys, L-arginine induced arginine net uptake, ornithine net efflux and urea in parallel with increased glutamine net uptake. On the contrary, L-arginine administration inhibited glutamine net uptake by the portal-drained viscera. The glutamine efflux from the hindquarter was not affected by Larginine. 


\section{Hemodynamics}

\subsection{Endotoxin}

In most septic patients hospitalized in the intensive care unit, an aggressive approach to the management of cardiovascular dysfunction in septic shock is warranted. During sepsis a relative hypovolemia often occurs due to reduced peripheral vascular resistance leading to a decrease in mean arterial pressure (MAP). Large volume fluid resuscitation provides therapy for the treatment of sepsis and septic shock aiming at restoration of intravascular volume and improvements in cardiac output (CO). Large bolus injections of endotoxin lead to a hypodynamic circulatory pattern (20) with normal or elevated peripheral vascular resistance and MAP and low $\mathrm{CO}$. In contrast, when smaller doses of endotoxin are infused, a hyperdynamic septic shock profile (i.e., a high $\mathrm{CO}$ to compensate for low peripheral resistance) is achieved, better resembling the human sepsis (20). In addition, a hyperdynamic septic shock profile occurs only when concomitant fluid replacement is provided (12). In contrast to the biphasic hemodynamic pattern characteristically seen in patients, most experimental animal models have failed to reproduce the early, hyperdynamic phase of sepsis and endotoxemia. We designed a standardized model of endotoxemia, aiming to reproduce a model of long-term, hypo- or normotensive sepsis with high $\mathrm{CO}$ achieved by resuscitation closely resembling the clinical situation. For that purpose, we designed a standardized model of endotoxemia, in which pigs were given a continuous i.v. infusion of endotoxin in parallel with fluid resuscitation. Body temperature, MAP and heart rate were monitored during endotoxin infusion and compared to saline-treated control pigs (chapter 6). Moreover, data on changes in $\mathrm{CO}$ obtained from another pig study are discussed in this chapter.

The $3^{\circ} \mathrm{C}$ increase in body temperature of the pigs that was observed at 24-h endotoxin infusion suggests the presence of a systemic inflammatory response. Endotoxin-induced cytokines are responsible for the fever observed during sepsis (49). In endotoxin-treated resuscitated pigs, an initial decrease in MAP was observed after start of endotoxin infusion, but an increase above control values was noticed at 3-4 h. After 8-h endotoxin infusion, MAP again returned to baseline values and was maintained at 24 hours. Heart rate gradually increased and remained high at $24 \mathrm{~h}$. Cardiac output progressively rose to levels above those of control and also remained high for the remainder of the 24-h observation period as additional data pointed out. With respect to MAP, the endotoxemic animals showed a biphasic response which is consistent with findings in continuously endotoxin-infused and resuscitated sheep (33). The initial decrease in MAP is consistent with the early, so-called "vasodilator" phase. We observed a preserved CO which contrasts with the $1^{\text {st }}$ phase in non-resuscitated subjects, which is characterized by reduced $\mathrm{CO}$ (5). Due to adequate volume replacement, the initial decrease in the MAP 
and peripheral vascular resistance is rapidly compensated by increases in heart rate and $\mathrm{CO}$. The compensatory phase during which the MAP is normalized to basal values may be recognized as the intermediate phase. During the late or $2^{\text {nd }}$ hyperdynamic phase, the so-called "vasocodilator phase", vascular resistance and MAP are decreased simultaneously with markedly elevated $\mathrm{CO}$. Fluid replacement strategy involves adjustment of the amount of fluid required for maintenance of MAP and the hypotension characteristic of the septic shock situation may be reversed by fluid resuscitation (17). The hemodynamic profile observed shows that a hyperdynamic circulation was elicited by prolonged, low dose infusion of endotoxin co-infused with saline.

The endotoxin infusion increased plasma flow to the portal-drained viscera and liver and tended to increase plasma flow to the hindquarter while at 1 and 4 days postendotoxemia, the plasma flow to the hindquarter was significantly elevated (chapter 6 and 4). Maintenance of organ plasma flow is characteristic of hyperdynamic sepsis and a high flow state is encountered when peripheral blood pooling is compensated by adequate volume replacement (17).

\subsection{Arginine intervention}

Excessive NO production by the NOS-II isoform in septic shock has been proposed to play a role in the pathogenesis of endotoxin-induced arteriolar vasodilatation, hypotension, and shock (26). Addition of L-arginine as substrate of NO, for that reason, may influence the endotoxin-induced changes in hemodynamics by promoting NO production under septicemic conditions. Indeed, L-arginine supplementation in septic but not in normal sheep was found to decrease the MAP, suggesting the induced NOS-IIisoform to play a role in systemic vasodilatation (28). In this model of sepsis, i.v. Larginine administration after an infusion period of $24 \mathrm{~h}$, accordingly, decreased MAP in endotoxemic pigs (chapter 7). L-Arginine infusion further increased measured $\mathrm{Cl}$ and $\mathrm{CO}$ at 4-4.5 $\mathrm{h}$ compared to endotoxemic controls which may constitute a mechanism to compensate for the reduced peripheral vascular resistance and MAP (not shown). Although NO-mediated dilator actions of L-arginine on peripheral and microvasculatur might be expected (26), neither intravenous, nor intragastric administration of L-arginine affected plasma flow to the organs. L-Arginine infused in humans at lower doses than in our experiments showed no vasodilator effect whereas higher doses increased peripheral flow, although this was under normal conditions excluding a NOS-II mediated effect (9). Treatment of septic rats with L-arginine analogue increase systemic, intestinal and portal flow (31) it was also found to increase pulmonary flow (1). Since non-selective NOS inhibitors were used, these effects may however be attributed to inhibition of constitutively formed NO. 


\section{Motility}

\subsection{Endotoxin}

Jejunal motility was measured during endotoxemia as a parameter of intestinal functioning. In critically ill patients, retardation or acceleration of gastrointestinal transit associated with generalized ileus or diarrhea are frequently observed intestinal disorders (46). Many factors such as anesthesia, surgical manipulation, infection, therapeutic agents and nutritional status contribute to an altered pattern of motility. Besides these exogenous factors, bacterial endotoxins and endotoxin-induced mediators like neuropeptides, cytokines and nitric oxide (NO) are main endogenous stimuli involved in the pathogenesis of motility disorders observed in endotoxin-induced septicemia $(34,37)$. Changes in motility pattern may strongly depend on the model of endotoxemia used. A single, sublethal dose of endotoxin was shown to temporarily disrupt fasting and postprandial canine gastrointestinal motility and transit (14). Such changes may be associated with a hypodynamic state of endotoxemia. Previously it was shown that single i.v. bolus of endotoxin (44) caused a biphasic motility pattern in the colon of dogs; MMC's (migrating motor complexes) in the initial phase (day 1 post-endotoxin) were shortened but lengthened during the later phase (day 2 and 3 post-endotoxin). These findings are similar to the biphasic motility pattern that was observed in non-resuscitated i.v. endotoxin treated piglets (27). In non-resuscitated endotoxemic subjects, reduced $\mathrm{CO}$ characterizes the initial phase of sepsis, due to lack of volume replacement. In our hyperdynamic model of endotoxemia sufficient fluid infusion was provided to endotoxin treated pigs to maintain $\mathrm{CO}$ and to endure a hyperdynamic sepsis profile. We observed increased MMC cycling frequency, MMC migration velocity and the motility index (calculated from contraction frequency and amplitude) of the jejunum (chapter 9) during 24-h endotoxin infusion and 1 day thereafter. Whether the dissimilarities in the recovery phase are related to preserved $\mathrm{CO}$ and MAP remains to be established. Elevated MMC migration velocity and motility index were maintained on the day after endotoxin challenge during feeding but recovered 4 days post-endotoxin infusion. Increased motility index and accelerated MMC migration in the jejunum of our continuously endotoxininfused pigs may not share the same characteristics of the motility changes in the stomach and colon. Whereas gastric emptying often is delayed, transit of luminal contents through the small intestine may be accelerated, whereas colonic passage is delayed. It remains to be established whether jejunal motility changes are comparable and occur consecutively with colonic motility changes that are observed in the pathogenesis of endotoxin-induced diarrhea and adynamic ileus. 


\subsection{Arginine intervention}

NANC neurons containing the NOS-I isoform mediate smooth muscle relaxation, inhibit slow wave amplitude and frequency (8) and coordinate the periodic cycling of the migrating motor complexes (MMC's) in the gut (41). Therefore, a minimal production of NO as a NANC inhibitory mediator may be essential for optimal intestinal motility functioning involving a basic requirement for this molecule. We hypothesized that motility disturbances observed in prolonged sepsis are associated with reduced L-arginine availability as a substrate for NO formation in the gut. Although some studies have been performed regarding L-arginine or NO donating compounds on the intestinal motility pattern under physiologic circumstances, the effects under septic conditions are unknown.

Arginine was infused intravenously to assess the effect of increasing the supply of NO precursor under conditions of sepsis. In the post-endotoxemic state, L-arginine was supplemented via the intragastric route by addition to the enteral diet. Intravenous administration of L-arginine during endotoxin infusion restored the endotoxin-induced increase in jejunal migrating motor complex (MMC) cycle frequency and migration velocity to control values of non-endotoxemic pigs (chapter 10). Furthermore, the incidence of diarrhea appeared to be lower in the L-arginine-treated than in the control endotoxemic pigs. Addition of intragastric L-arginine one day after cessation of endotoxin infusion had similar effects. L-Arginine intervention could be associated with a return to normal of the jejunal motility including reduction of the increased MMC occurrence and speed along the jejunum restoring the motility index to normal values. A similar inhibitory effect of L-arginine on the MMC contraction frequency was observed in dogs (29), although under normal physiological circumstances. Moreover, NO donating compounds inhibited spontaneous contractile activity in the rat jejunum (24) suggesting an inhibitory role for NOS-I-derived NO on the motility activity. During endotoxin infusion, but not thereafter, NO synthesis by the portal-drained viscera was induced. L-Arginine supplementation during and after endotoxin challenge resulted in an additional increase in visceral NO production. This corresponds with studies in which the L-arginine availability determined the rate of NO synthesized from the NOS $(7,50)$. Deranged motility appears not proportionally related to rates of NO produced. Whereas endotoxin induced expression of NOS-II, it simultaneously inhibited the constitutive NOS-I and III isoforms (2). We therefore hypothesized that the impaired synthesis rates of NO from the NOS-I isoform rather than the high NO production rates from the NOS-II isoform underlie the derangements in motility observed during and after endotoxemia. Restoration in motility pattern (i.e., MMC occurrence, MMC migration velocity and motility index) by Larginine supplementation may be achieved by provision of sufficient substrate to improve NO synthesis by the neuronal NOS (NOS-1) isoform associated with NANC innervation. 


\section{References}

1. Ayuse, T., N. Brienza, J. P. Revelly, J. K. Boitnott, and J. L. Robotham. Role of nitric oxide in porcine liver circulation under normal and endotoxemic conditions. I Appl Physiol 78: 1319-29, 1995

2. Beach, P. K., D. A. Spain, T. Kawabe, P. D. Harris, and R. N. Garrison. Sepsis increases NOS-2 activity and decreases non-NOSmediated acetylcholine-induced dilation in rat aorta. J Surg Res 96: 17-22., 2001.

3. Bohe, J., J. F. Low, R. R. Wolfe, and M. J. Rennie. Latency and duration of stimulation of human muscle protein synthesis during continuous infusion of amino acids. $J$ Physiol 532: 575-9., 2001.

4. Bower, R. H. Nutrition during critical illness and sepsis. New Horiz 1: 348-52., 1993.

5. Brackett, D. J., C. F. Schaefer, P. Tompkins, L. Fagraeus, L. J. Peters, and M. F. Wilson. Evaluation of cardiac output, total peripheral vascular resistance, and plasma concentrations of vasopressin in the conscious, unrestrained rat during endotoxemia. Circ Shock 17: 273-84, 1985.

6. Breuille, D., M. Arnal, F. Rambourdin, G. Bayle, D. Levieux, and C. Obled. Sustained modifications of protein metabolism in various tissues in a rat model of long-lasting sepsis. Clin Sci (Colch) 94: 413-23, 1998.

7. Bune, A. J., J. K. Shergill, R. Cammack, and H. T. Cook. L-Arginine depletion by arginase reduces nitric oxide production in endotoxic shock: an electron paramagnetic resonance study. FEBS Lett 366: 127-130, 1995.

8. Calignano, A., B. J. R. Whittle, M. DiRosa, and $S$. Moncada. Involvement of endogenous nitric oxide in the regulation of rat intestinal motility in vivo. Eur J Pharmacol 37: 273-276, 1992.

9. Calver, A., J. Collier, and P. Vallance. Dilator actions of arginine in human peripheral vasculature. Clin Sci (Colch) 81: 695-700, 1991.

10. Campbell, I. T. Limitations of nutrient intake. The effect of stressors: trauma, sepsis and multiple organ failure. Eur J Clin Nutr 53 Suppl 1: S143-7., 1999.

11. Cerra, F. B. Hypermetabolism, organ failure, and metabolic support. Surgery 101: 1-14., 1987.

12. Cholley, B. P., R. M. Lang, D. S. Berger, C. Korcarz, D. Payen, and S. G. Shroff. Alterations in systemic arterial mechanical properties during septic shock: role of fluid resuscitation. Am J Physiol 269: H375-84., 1995.
13. Clowes, G. H., Jr., E. Hirsch, B. C. George, L. M. Bigatello, J. E. Mazuski, and C. A. Villee, Jr. Survival from sepsis. The significance of altered protein metabolism regulated by proteolysis inducing factor, the circulating cleavage product of interleukin-1. Ann Surg 202: 446-58., 1985.

14. Cullen, J. J., D. K. Caropreso, and K. S. Ephgrave. Effect of endotoxin on canine motility and transit. J Surg Res 58: 90-95, 1995.

15. de Blaauw, I., N. E. Deutz, and M. F. von Meyenfeldt. Muscle protein and amino acid turnover in rats in vivo: effects of short-term and prolonged starvation. Clin Sci (Colch) 90: 457-66., 1996.

16. Dejong, C. H. C., N. E. P. Deutz, and P. B. Soeters. Inter-organ nitrogen exchange during prolonged starvation in the rat. J Clin Nutr Gastroenterol 6: 176-183, 1991.

17. D'Orio, V., P. Mendes, P. Carlier, M. Fatemi, and R. Marcelle. Lung fluid dynamics and supply dependency of oxygen uptake during experimental endotoxic shock and volume resuscitation. Crit Care Med 19: 955-62., 1991.

18. Driscoll, D. F., and G. L. Blackburn. Total parenteral nutrition 1990. A review of its current status in hospitalised patients, and the need for patient-specific feeding. Drugs 40: 346-63., 1990.

19. Evoy, D., M. D. Lieberman, T. J. Fahey, 3rd, and J. M. Daly. Immunonutrition: the role of arginine. Nutrition 14: 611-7, 1998.

20. Fink, M. P., and S. O. Heard. Laboratory models of sepsis and septic shock. J Surg Res 49: 186-196, 1990.

21. Hasselgren, P. O. Glucocorticoids and muscle catabolism. Curr Opin Clin Nutr Metab Care 2: 201-5., 1999.

22. Hasselgren, P. O., and J. E. Fischer. Counterregulatory hormones and mechanisms in amino acid metabolism with special reference to the catabolic response in skeletal muscle. Curr Opin Clin Nutr Metab Care 2: 9-14., 1999.

23. Higashiguchi, T., Y. Noguchi, A. Noffsinger, J. $E$. Fischer, and P. O. Hasselgren. Sepsis increases production of total secreted proteins, vasoactive intestinal peptide, and peptide $Y Y$ in isolated rat enterocytes. Am J Surg 168: 251-6., 1994.

24. Izzo, A. A., N. Mascolo, P. Maiolino, and F. Capasso. Nitric oxide-donating compounds and cyclic GMP depress the spontaneous contractile activity of the isolated rabbit jejunum. Pharmacology 53: 109-13, 1996. 
25. James, J. H., C. H. Fang, S. J. Schrantz, P. O. Hasselgren, R. J. Paul, and J. E. Fischer. Linkage of aerobic glycolysis to sodiumpotassium transport in rat skeletal muscle. Implications for increased muscle lactate production in sepsis. $J$ Clin Invest 98: 2388-97, 1996.

26. $\mathrm{Li}, \mathrm{H}$., and $U$. Forstermann. Nitric oxide in the pathogenesis of vascular disease. I Pathol 190: 244-54., 2000.

27. Li, J. X., J. R. Oliver, and J. B. Philips, 3rd. Endotoxin induces biphasic alterations in small intestinal myoelectric activity in fasted newborn piglets. Pediatr Res 40: 822-6, 1996.

28. Lorente, J. A., M. A. Delgado, C. Tejedor, E. Mon, M. Hervas, T. Pascual, P. FernandezSegoviano, G. Rieppi, A. Soler, D. Ayuso, and A. Esteban. Modulation of systemic hemodynamics by exogenous L-arginine in normal and bacteremic sheep. Crit Care Med 27: 2474-9, 1999.

29. Maczka, M., P. Thor, K. Lorens, and S. J. Konturek. Nitric oxide inhibits the myoelectric activity of the small intestine in dogs. J Physiol Pharmacol 44: 31-42, 1993.

30. Mainous, M. R., E. F. Block, and E. A. Deitch. Nutritional support of the gut: how and why. New Horiz 2: 193-201., 1994.

31. Martin, C. M., and W. J. Sibbald. Modulation of hemodynamics and organ blood flow by nitric oxide synthase inhibition is not altered in normotensive, septic rats. Am J Respir Crit Care Med 150: 1539-44., 1994.

32. Metges, C. C. Contribution of microbial amino acids to amino acid homeostasis of the host. $J$ Nutr 130: 1857S-64S., 2000.

33. Meyer, J., C. S. Cox, D. N. Herndon, H. Nakazawa, C. W. Lentz, L. D. Traber, and D. L. Traber. Heparin in experimental hyperdynamic sepsis. Crit Care Med 21: 84-9., 1993.

34. Meyer, W. Clinical aspects, pathogenesis and therapy of shock due to sepsis caused by gram-negative organisms. Schweiz Med Wochenschr 96: 137-43, 1966.

35. Millward, D. J., P. J. Garlick, D. O. Nnanyelugo, and J. C. Waterlow. The relative importance of muscle protein synthesis and breakdown in the regulation of muscle mass. Biochem J 156: 185-8., 1976.

36. Moldawer, L. L., S. J. O'Keefe, A. Bothe, Jr., B. $R$. Bistrian, and $G$. L. Blackburn. In vivo demonstration of nitrogen-sparing mechanisms for glucose and amino acids in the injured rat. Metabolism 29: 173-80., 1980.

37. Moon, H. W. Pathogenesis of enteric diseases caused by Escherichia coli. Adv Vet Sci Comp Med 18: 179-211, 1974.
38. Newsholme, E. A., and A. R. Leech, Biochemisty for the Medical Sciences. New York: John Wiley \& Sons, 1983. p. 1-952.

39. Pastor, C. M., S. M. Morris, Jr., and T. R. Billiar. Sources of arginine for induced nitric oxide synthesis in the isolated perfused liver. Am J Physiol 269: G861-G866, 1995.

40. Remesy, C., C. Moundras, C. Morand, and C. Demigne. Glutamine or glutamate release by the liver constitutes a major mechanism for nitrogen salvage. Am J Physiol 272: G257-64., 1997.

41. Sarna, S. K., M. F. Otterson, R. P. Ryan, and $V$. E. Cowles. Nitric oxide regulates migrating motor complex cycling and its postprandial disruption. Am J Physiol 265: G759-G766, 1993.

42. Schott, C. A., G. A. Gray, and J. C. Stoclet. Dependence of endotoxin-induced vascular hyporeactivity on extracellular L-arginine. $\mathrm{Br} J$ Pharmacol 108: 38-43., 1993.

43. Scornik, O. A. Role of protein degradation in the regulation of cellular protein content and amino acid pools. Fed Proc 43: 1283-8., 1984.

44. Spates, S. T., J. J. Cullen, K. S. Ephgrave, and M. M. Hinkhouse. Effect of endotoxin on canine colonic motility and transit. $J$ Gastrointest Surg 2: 391-8, 1998.

45. Tappy, L., M. Berger, J. M. Schwar, M. McCamish, J. P. Revelly, P. Schneiter, E. Jequier, and R. Chiolero. Hepatic and peripheral glucose metabolism in intensive care patients receiving continuous high- or low-carbohydrate enteral nutrition. JPEN J Parenter Enteral Nutr 23: 260-7; discussion 267-8., 1999.

46. Thompson, J. S. The intestinal response to critical illness. Am J Gastroenterol 90: 190200, 1995.

47. Vary, T. C. Inter-organ protein and carbohydrate metabolic relationships during sepsis: necessary evils or uncanny coincidences? Curr Opin Clin Nutr Metab Care 2: 235-42, 1999.

48. Vary, T. C., and S. R. Kimball. Regulation of hepatic protein synthesis in chronic inflammation and sepsis. Am J Physiol 262: C445-52, 1992.

49. Waage, A., P. Brandtzaeg, T. Espevik, and A. Halstensen. Current understanding of the pathogenesis of gram-negative shock. Infect Dis Clin North Am 5: 781-91., 1991.

50. Wu, G., N. E. Flynn, S. P. Flynn, C. A. Jolly, and $P . K$. Davis. Dietary protein or arginine deficiency impairs constitutive and inducible nitric oxide synthesis by young rats. $J$ Nutr 129: 1347-54, 1999. 
CHAPTER 12

SUMMARYISAMENVATTING 


\section{Summary}

Sepsis is characterized by the presence of bacteria or their cell wall components (such as endotoxin) in the circulation. These endotoxins induce an immune response, which aims to eradicate the bacterial infection. Owing to the inadequate immune response in immunocompromised individuals, bacteria can break out into the circulation and an infection may develop into sepsis. After exposure to bacterial endotoxin or certain cytokines, expression of the inducible nitric oxide synthase (NOS-II) occurs in a wide variety of tissues. This NOS-II enzyme produces large amounts of nitric oxide (NO) for long periods and is implicated in the pathophysiologic changes manifest in sepsis. The role of NO in endotoxin induced septic shock remains controversial; NO is a crucial mediator of organ defense, but, paradoxically, it also contributes to tissue injury in certain situations. Presumably, in this, the amount of NO synthesized is of importance. Moderate NO synthesis results in optimal defeat of the infection whereas insufficient or uncontrolled NO production results in tissue damage. The release of arginine as a substrate of NO over a prolonged period of time may be useful in overcoming the reduced plasma arginine levels as occur in prolonged sepsis. Provision of arginineenriched enteral formulas has already proven to be beneficial under certain catabolic situations such as postoperative stress and severe sepsis. Nevertheless, the enteral diets used in these studies consisted of multiple components as a result of which a beneficial affect may not uniquely be attributed to arginine as such. As a model of sepsis and recovery, pigs were continuously infused endotoxin and, subsequently, enterally fed. Endotoxin infusion was accompanied by generous fluid infusion to replenish intravascular volume losses en to elicit a hyperdynamic circulation. In this thesis, the effects on the motility pattern in the jejunum, the protein synthesis and degradation and the arginine and NO metabolism in the different organs were described. Subsequently, the effects of arginine treatment on these aspects were evaluated. The sepsis model was, besides increased body temperature, characterized by maintained to increased perfusion of the organs, maintained mean arterial blood pressure and increased cardiac output. These are all characteristics of the hyperdynamic circulation encountered in septic patients receiving modern intensive care. The unchanged protein synthesis and accelerated protein breakdown in muscle during endotoxin infusion attribute to the net protein loss which constitutes a main hallmark of the septic condition. The protein synthesis and degradation of the portal-drained viscera remained unchanged during endotoxemia. The mobilization of amino acids and lactate from the periphery provide substrate for the liver. The increase in net amino acid and lactate uptake by the liver during endotoxemia was related with increased liver gluconeogenesis. During sepsis, the liver acts as a main provider of glucose which serves as substrate for immune cells and, organs such as the spleen and intestines which are to a large extent involved in the host's immune response. Increased net glucose uptake and net lactate release by the 
muscle and portal-drained viscera reflect increased glycolysis in these organs. The increase in liver protein synthesis that was observed during endotoxin infusion presumably reflects enhanced production of acute phase proteins, which play an important role in the immune response of the host. In the recovery phase of endotoxemia during enteral feeding, net protein loss was no longer present in the muscle but net protein gain due to increase in protein synthesis exceeding the increase in protein degradation. This may reflect replenishment of the, during endotoxemia, degraded muscle proteins. While liver protein synthesis was sustained in the recovery phase of endotoxemia, increased liver gluconeogenesis was no longer observed. In the recovery situation, an increase in net release of amino acids in the portal vein was observed. Since the protein synthesis and breakdown in the portal-drained viscera were not altered, microbial amino acid synthesis could probably play a role in this amino acid release. Endotoxin exposure resulted in increased rate of $\mathrm{N}_{2}$-arginine to $\mathrm{N}_{1}$-citrulline conversion, indicating stimulated NO production by the portal-drained viscera and the liver. Most likely, this is caused by cytokines and endotoxin induced NOS-II activity. Endotoxin infusion accelerated the migrating motor complex (MMC) migration and MMC cycling frequency along the jejunum, which both were maintained the following day postendotoxemia. Whereas gastric emptying and colonic passage often is delayed during sepsis, it appears that transit of luminal contents through the small intestine are accelerated.

Both during and after endotoxin exposure, L-arginine administration enhanced protein synthesis and degradation in the muscle and reduced the increase in liver protein synthesis and degradation. Although L-Arginine treatment diminished the protein turnover at whole body level, it resulted in, neither a negative, nor a positive net protein balance. This possibly reflects limited liver acute phase response. L-Arginine treatment increased the total appearance and disappearance of arginine at whole body level. Increased arginine degradation and, to a lesser extent, decreased arginine de novo synthesis by the kidneys, were a involved in the regulation of arginine homeostasis. L-Arginine administration during and after endotoxemia increased the NO production by the portaldrained viscera, liver and kidneys. This suggests that the NO synthesis rate is dependent on the arginine availability. L-Arginine infusion during and after endotoxemia restored the endotoxin-induced hypermotility. Whether there is a relation between the increased visceral NO production elicited by arginine and the improved motility function remains to be established. Based on the results of this thesis it appears justified to conclude that the NO production by the organs is rate-dependent on the arginine appearance in the circulation. L-Arginine supplementation under conditions of prolonged sepsis may exert a beneficial effect on the immune function by means of compensating the reduced arginine plasma concentrations and, thereby, improve the relative NO synthesis capacity. 


\section{Samenvatting}

Sepsis wordt gekenmerkt door de aanwezigheid van bacteriën of bacteriële celwand bestanddelen (zoals endotoxine) in de bloedbaan. Deze celwandproducten zetten een immuunreactie in gang die er op gericht is om de bacteriële infectie te elimineren. Doordat de immuun response in immuun gecompromitteerde individuen ontoereikend is, kunnen bacteriën doorbreken naar de bloedbaan en kan een infectie zich tot sepsis ontwikkelen. $\mathrm{Na}$ blootstelling aan bacteriële endotoxines of bepaalde cytokines, vindt er expressie van het induceerbare stikstof oxide synthase enzym (NOS-II) plaats in verschillende weefsels. Dit NOS-II enzym produceert grote hoeveelheden stikstof oxide (NO) voor lange periodes en is geïmpliceerd in de pathofysiologische veranderingen die manifest zijn tijdens sepsis. De rol van NO gedurende endotoxine geïnduceerde septische shock is controversieel; NO is een cruciale mediator in orgaan afweer, maar, paradoxaal genoeg, kan het ook bijdragen tot weefselschade in bepaalde situaties. Vermoedelijk is de hoeveelheid NO die wordt aangemaakt hierbij van belang. Gematigde NO synthese resulteert in een optimale bestrijding van de infectie terwijl onvoldoende of juist ongecontroleerde NO productie tot weefselschade leidt. De afgifte van arginine als een substraat van NO over een langere tijdsperiode zou nuttig kunnen zijn in de tegemoetkoming van de verlaagde arginine plasma concentraties die optreden tijdens langdurige sepsis Toediening van arginine-verrijkte enterale formules bleek reeds gunstig in bepaalde katabole situaties zoals postoperatieve stress en ernstige sepsis waarbij het de stikstof balans en de immuun status verbeterde. Desalniettemin bestond het enterale dieet wat in deze studies werd gebruikt uit meerder componenten waardoor een gunstig effect niet enkel aan arginine kan worden toegewezen. Als een model van sepsis en herstel van sepsis werden varkens continu geïnfundeerd met endotoxine en, vervolgens, enteraal gevoed. Endotoxin infusie werd begeleid door genereus vocht infusie om het intravasculaire volume verlies aan te vullen en een hyperdynamische circulatie te bewerkstelligen. In dit proefschrift zijn de effecten op het motiliteitspatroon van het jejunum, de eiwitsynthese en -degradatie en het arginine en NO metabolisme van de verschillende organen beschreven. Vervolgens werden de effecten van arginine behandeling op deze aspecten beschouwd. Het sepsis model werd, behalve door toegenomen lichaamstemperatuur, gekenmerkt door gehandhaafde tot toegenomen perfusie van de organen, gehandhaafde arteriële bloeddruk en toegenomen hartslag capaciteit. Dit zijn allemaal karakteristieken van de hyperdynamische circulatie zoals die wordt waargenomen in septische patiënten die moderne eerste hulp krijgen. De onveranderde eiwitsynthese en versnelde eiwitafbraak in de spier tijdens endotoxine infusie dragen bij tot het netto eiwit verlies wat zo kenmerkend is voor de septische conditie. De eiwitsynthese en -afbraak in de portaal-gedraineerde inwendige organen 
bleef onveranderd gedurende endotoxinemie. De mobilisatie van aminozuren en lactaat vanuit de periferie voorziet de lever van substraat. De toename in aminozuur en lactaat opname door de lever gedurende endotoxinemie was gerelateerd aan verhoogde mate van lever glucose nieuwvorming. Tijdens sepsis is de lever een belangrijke leverancier van glucose wat als substraat dient voor immuuncellen en, organen zoals darmen en milt die in grote mate betrokken zijn bij de immuun afweer van de gastheer. Verhoogde netto glucose opname en lactaat afgifte door de spier en de portaal-gedraineerde inwendige organen, suggereren dat de glycolyse verhoogd is in deze organen. De tijdens endotoxine infusie waargenomen toename in lever eiwitsynthese, reflecteert hoogstwaarschijnlijk de synthese van acute fase eiwitten welke een belangrijke rol spelen in de immuun afweer van de gastheer. In de herstelfase van endotoxinemie gedurende enteraal voeden, is in de spier geen netto eiwit verlies meer aanwezig maar netto eiwit winst dankzij een toename in eiwitsynthese die de toename in eiwitafbraak overtreft. Dit duidt er waarschijnlijk op dat de spiereiwitten die tijdens endotoxinemie zijn afgebroken worden hersteld. Terwijl toegenomen lever eiwitsynthese nog aanhield in de herstel fase van endotoxinemie, werd geen toegenomen lever glucose nieuwvorming meer waargenomen. In de herstel situatie werd een toename in netto afgifte van aminozuren in de portale vene waargenomen. Aangezien de eiwitsynthese en -afbraak in de portaal-gedraineerde inwendige organen niet veranderde zou microbiële aminozuur synthese een rol kunnen spelen in deze vrijlaat van aminozuren. Blootstelling aan endotoxine resulteerde in verhoogde mate van $\mathrm{N}_{2}$-arginine naar $\mathrm{N}_{1}$-citrulline conversie, wat duidt op een gestimuleerde NO productie door de portaal-gedraineerde inwendige organen en lever. Vermoedelijk ligt hier een door cytokines en endotoxine geinduceerde NOS-Il activiteit aan ten grondslag. Endotoxine infusie versnelde de migratie snelheid van het migrerend motor complex (MMC) en de MMC verschijningsfrequentie in het jejunum welke beide nog aanwezig waren de dag na endotoxine behandeling. Terwijl gedurende sepsis de maaglediging en passage door het colon vertraagd zijn, lijkt het er dus op dat endotoxine de passage snelheid van de darminhoud door het jejunum doet toenemen.

Zowel gedurende als na endotoxine behandeling verhoogde L-arginine toediening de eiwitsynthese en -degradatie in de spier en reduceerde het de toename in lever eiwitsynthese en -afbraak. Hoewel L-arginine de eiwit omzetsnelheid op heel lichaamsniveau verminderde, realiseerde het netto in noch een positieve, noch een negatieve eiwit balans. Mogelijkerwijs reflecteert dit een beperkte lever eiwit acute fase response. L-Arginine behandeling verhoogde de totale verschijning en verdwijning van arginine op heel lichaamsniveau. Toegenomen arginine degradatie en, in mindere mate, afgenomen arginine nieuwvorming door de nier waren betrokken in de regulatie van het arginine evenwicht. L-Arginine toediening gedurende en na endotoxinemie verhoogde de 
NO productie door de portaal-gedraineerde inwendige organen, lever en nieren. Dit suggereert dat de NO synthese snelheid afhankelijk is van de arginine beschikbaarheid. L-Arginine infusie gedurende en na endotoxinemie herstelde de endotoxinegeïnduceerde hypermotiliteit. Of er een samenhang is met de verhoogde darm NO productie bewerkstelligd door arginine toediening en de verbeterde motiliteitsfunctie is iets wat nog een open staat.

Tenslotte lijkt op grond van de gegevens in dit proefschrift de conclusie gerechtvaardigd dat de NO productie door de organen afhankelijk is van de arginine aanwezigheid in de circulatie. Arginine aanvulling onder condities van langdurige sepsis zou mogelijk een gunstig effect kunnen uitoefenen op de immuun functie middels compensatie van de verlaagde arginine plasma concentraties en, daarbij, de relatieve NO synthese capaciteit verbeteren. 




\section{LIST OF ABBREVIATIONS}

\begin{tabular}{|c|c|}
\hline$\alpha-A N$ & = alpha-amino nitrogen \\
\hline BCAA & $=$ branched-chain amino acid \\
\hline BCKA & = Branched-chain alpha-keto acid \\
\hline bw & $=$ body weight \\
\hline CAT & = cationic amino acid transporter \\
\hline cGMP & = cyclic guanosine monophosphate \\
\hline CRP & $=\mathrm{C}$ reactive protein \\
\hline D & $=$ disposal \\
\hline$E_{A B S}$ & $=$ absorption from enterally infused meal \\
\hline ET & $=$ endotoxin \\
\hline FE & $=$ fractional extraction \\
\hline GAA & = guanidino acetic acid \\
\hline $\mathrm{GH}$ & $=$ growth hormone \\
\hline GNAA & $=$ gluconeogenic amino acids \\
\hline $\mathrm{HCO}_{3}^{-}$ & $=$bicarbonate \\
\hline ICU & $=$ intensive care unit \\
\hline IF & $=$ interferon \\
\hline IL & $=$ interleukin \\
\hline$H Q$ & $=$ hindquarter \\
\hline 1 & $=$ tracer infusion rate \\
\hline LC-MS & $=$ liquid chromatography-mass spectrometry \\
\hline LPS & $=$ lipopolysaccharide \\
\hline MAP & $=$ mean arterial blood pressure \\
\hline NANC & $=$ non-adrenergic non-cholinergic \\
\hline NB & $=$ net balance \\
\hline NO & $=$ nitric oxide \\
\hline NOx & $=$ sum of nitrite and nitrate \\
\hline OKG & $=$ ornithine alpha-ketoglutarate \\
\hline$P$ & $=$ production \\
\hline$P_{\text {END }}$ & $=$ endogenous production \\
\hline $\mathrm{P}_{\mathrm{ND}}$ & $=$ non-disposed production \\
\hline $\mathrm{PaO}_{2 / \mathrm{CO} 2}$ & $=$ arerial $\mathrm{O}_{2} / \mathrm{CO}_{2}$ pressure \\
\hline $\mathrm{PAH}$ & $=$ para-aminohippuric acid \\
\hline PDV & = portal-drained viscera \\
\hline PG & $=$ prostaglandin \\
\hline Q Ra & $=$ whole body rate of appearance \\
\hline SA & $=$ specific activity \\
\hline TNF & $=$ tumor necrotising factor \\
\hline TTR & $=$ tracer-to-tracee ratio \\
\hline$V_{\max }$ & $=$ maximal transport rate \\
\hline
\end{tabular}




\section{LIST OF ENZYMES CODES}

\begin{tabular}{|c|c|c|}
\hline$A-I$ & [EC 3.5.3.1] & : Hepatic arginase \\
\hline$A-I I$ & [EC 3.5.3.1] & : Non-hepatic arginase \\
\hline ADC & [EC 4.1.1.19] & : Arginine decarboxylase \\
\hline Agmatinase & [EC 3.5.3.11] & : Agmatine urohydrolase \\
\hline ASL & [EC 4.3.2.1] & : Argininosuccinate lyase \\
\hline ASS & [EC 6.3.4.5] & : Argininosuccinate synthetase \\
\hline AT & [EC 2.1.4.1] & : Arginine:glycine amidinotransferase \\
\hline CPS & {$[E C$ 6.3.4.16] } & : Carbamoylphosphate synthetase \\
\hline GMT & [EC 2.1.1.2] & : S-adenosylmethionine:guanidinoacetate methyltransferase \\
\hline GS & [EC 2.3.1.1.] & : N-acetylglutamate synthetase \\
\hline NOS & [EC 1.14.13.39] & : Nitric oxide synthase \\
\hline P5C-D & [EC n.a.] & : Pyrroline-5-carboxylate dehydrogenase \\
\hline P5C-R & [EC n.a.] & : Pyrroline-5-carboxylate reductase \\
\hline P5C-S & [EC n.a.] & : Pyrroline-5-carboxylate synthetase \\
\hline PDG & [EC 3.5.1.2.] & : Phosphate-dependent glutaminase \\
\hline $\mathrm{PO}$ & [EC n.a.] & : Proline oxidase \\
\hline OAT & [EC 2.6.1.13] & : Ornithine aminotransferase \\
\hline отC & [EC 2.1.3.3] & : Ornithine transcarbamoylase \\
\hline ODC & [EC 4.1.1.17] & : Ornithine decarboxylase \\
\hline
\end{tabular}




\section{DANKWOORD}

Hoewel het promoveren weliswaar grotendeels een solitaire aangelegenheid is, zijn er toch vele mensen onmisbaar geweest bij het tot stand komen van dit proefschrift. Bij de mensen die ik nu ga opsommen zal ik daarom ook ongetwijfeld niet iedereen hebben genoemd.

Om te beginnen wil ik Mick bedanken voor het in goede banen leiden van het onderzoek, voor het in mij gestelde vertrouwen en voor zijn aanstekelijke enthousiasme. Ik ben hem erg erkentelijk voor de hulp die ik kreeg bij de, in recordtempo, uitgevoerde operaties; als chirurgische leermeester bracht hij me snel de kneepjes van het vak bij. Van veler waarde was Gabrie die instond bij praktische problemen en menig sukkelend varken over de streep heeft getrokken. Dankzij haar hulp bij de verzorging van de varkens stond ik er niet alieen voor in de weekenden. Dan was er nog de gezelligheid van "het lab" die ik niet snel zal vergeten ondanks dat ik vaak in het "groene hok" zat. Onder leiding van Dennis bleek de LCQ niet alleen monsters te kunnen meten maar ook CD's te kunnen branden. Helaas ging hij naar Finnigan. Hans bleek altijd bereid een helpende hand toe te steken wanneer de MIRA weer eens verstek liet gaan en bleek altijd een opbeurend woord klaar te hebben in tijden van tegenslag. Daarbij mag ook Jean niet worden vergeten die altijd verbazingwekkend vrolijk bleef wanneer de zoveelste kolom naar de knoppen was geholpen door mijn vieze varkensmonsters. De meeste steun heb ik aan Marcella gehad. Het samen schelden hield de moraal hoog en het meester worden van de "onverklaarbare getallen" in de spreadsheets was ons voornaamste doel. Onze afgelopen jaren vertoonden opvallend veel parallellen, misschien de reden waarom ze ons door elkaar haalden. Daarbij waren er ook nog mijn kamergenoten Juanita en Yvonne die misschien blij zijn dat de rust nu weergekeerd is. Tussen de middag konden de frustraties op de squashbaan worden afgereageerd, bedankt Jessica en Anita. Met name wil ik ook de dierverzorgers Huub, Joyce en May van het CPV bedanken die er voor gezorgd hebben dat de OK's altijd vlot en strak verliepen. Bernadette, Carlo, Cees, Henk, Ivo en Mariëlle wil ik bedanken voor de congres-gezelligheid, Karel, Martin en Martijn voor de samenwerking bij de varkensproeven en Steven, die met zijn voortvluchtige varkens zorgde voor mooi spektakel. Mijn paranimf Charlotte wil ik bedanken voor een gezellige tijd in Maastricht. De suggesties voor verbeteringen van mijn stukken door Prof. Dr. W.A.H. Lamers, Dr. A.J. Meijer en Prof. Dr. P.B. Soeters, werden zeer gewaardeerd. Zij hebben mij daarmee onnoemelijk geholpen. Prof. Dr. P.B. Soeters, zijn enthousiasme voor basaal onderzoek was de pijler waarop de brug tussen het laboratoriumonderzoek en de kliniek rustte. Mijn arginine junior-collega's Karin en Wouter bedank ik voor het nemen van de vroege trein naar Maastricht voor de NWO- 
bijeenkomsten. Yvette, jouw deskundigheid van darmmotoriek heeft me buitengewoon veel verheldering gebracht op dit gebied. Van je nauwkeurigheid heb ik wat kunnen leren. Jan de Ruijter, met de uitleg die je me gaf werd statistiek toch opeens eenvoudiger dan ik dacht. Met name wil ook Monique bedanken, die met haar weergaloze inzet en organisatie onmisbaar bleek voor het regelen "op afstand". Daarbij waren er nog mijn collega's en al die genen die ik niet bij name heb genoemd maar wel bewust of onbewust een belangrijke bijdrage hebben geleverd bij het tot stand brengen van dit proefschrift. Dan wil ik nog mijn zus en Alex "the brilliant disc doctor" bedanken, en mijn ouders wil ik vermelden voor hun onvoorwaardelijke steun. En natuurlijk Bartl, mijn mental coach, zonder hem was dit alles nooit gelukt. 


\section{LIST OF PUBLICATIONS}

\section{Papers presented in this thesis}

- Bruins MJ, Soeters PB, Deutz NEP. Alterations in organ protein turnover and substrate fluxes during endotoxemia. Submitted: Ann Surg.

- Bruins MJ, Soeters PB, Deutz NEP. Endotoxemia affects organ protein metabolism differently during prolonged feeding in the pig. J Nutr 130, 3003-3013, 2000.

- Bruins MJ, Soeters PB, Lamers WH, Deutz NEP. L-Arginine supplementation in the pig decreases liver protein turnover and increases hindquarter protein turnover both during and after endotoxemia. Accepted: Am J Clin Nutr, 2001.

- Bruins MJ, Lamers WH, Soeters PB, Meijer AJ, Deutz NEP. In vivo measurements of organ nitric oxide production during hyperdynamic endotoxemia in pigs determined from stable isotope conversion. Submitted: $\mathrm{Br} J$ Pharmacol.

- Bruins MJ, Soeters PB, Lamers WH, Meijer AJ, Deutz NEP. L-Arginine supplementation increases organ nitric oxide production in a pig model of hypermetabolic endotoxemia. In press: Crit Care Med, 2001.

- Bruins MJ, Lamers WH, Meijer AJ, Deutz NEP. Effects of arginine supplementation on organ urea cycle intermediate metabolism in endotoxemic pigs. Submitted: J Nutr.

- Bruins MJ, Luiking YC, Soeters PB, Akkermans, LMA, Deutz NEP. The effect of prolonged hyperdynamic endotoxemia on jejunal motility in fasted and enterally fed pigs. Submitted: Ann Surg.

- Bruins MJ, Luiking YC, Soeters PB, Deutz NEP. Effects of long-term intravenous and intragastric L-arginine intervention on jejunal motility and visceral nitric oxide production in the endotoxemic pig. Submitted: Gut.

\section{Other papers}

- Deutz NEP, Bruins MJ, Soeters PB. Infusion of soy and casein protein meals affects interorgan amino acid metabolism and urea kinetics differently in pigs. $J$ Nutr 128: 24352445, 1998.

- Soeters PB, Bruins MJ, van Acker BAC, Dejong CHC, Deutz NEP. Stress response and Protein kinetics. Jap J Surg Met Nutr 25 (4), 237-245, 2001. 


\section{Book chapters}

- Bruins MJ, Soeters PB, Deutz NEP. The biological effects of soy and casein protein meals on liver amino acid metabolism and urea kinetics in pigs. Digestive physiology in pigs. EAAP Publication no. 88, Saint Malo, France, 1997.

\section{Congress publications}

- Bruins MJ, Soeters PB, Deutz NEP. Increased in vivo liver nitric oxide production during endotoxin challenge in pigs. P Nutr Soc 58: (4), 167A-167A, nov 1999.

- Bruins MJ, Soeters PB, Deutz NEP. Increased muscle, gut and liver protein kinetics during endotoxin challenge in pigs. FASEB J 13: (4), A81-A81 Part 1 Suppl. S, mar 12 1999.

- Bruins MJ, Soeters PB, Deutz NEP. Increased in vivo liver nitric oxide production during endotoxin challenge in pigs. FASEB J 13: (4), A102-A102 Part 1 Suppl. S, mar 121999.

- Bruins MJ, Soeters PB, Deutz NEP. Increased Cori cycling after endotoxin challenge in pigs. Hepatology 28: (4), 611A-611A Part 2 Suppl. S, oct 1998.

- Bruins MJ, Soeters PB, Deutz NEP. Increased in vivo liver nitric oxide production during endotoxin challenge in pigs. Gastroenterology 114: (4), A355-A355 Part 2 Suppl. S, apr 151998.

- Bruins MJ, Soeters PB, Deutz NEP. Increased liver protein synthesis after a casein protein meal in comparison to a soy protein meal. Gastroenterology 112: (4), A1233A1233, 2, Suppl. S, apr 1997.

- Bruins MJ, Deutz NEP, Soeters PB. Meting van de kwaliteit van eiwitten in een in vivo varkensmodel. Voeding 58: (5), 30, 1997.

- Bruins MJ, Deutz NEP, Soeters PB. Toegenomen levereiwitsynthese na een caseineeiwitmaaltijd in vergelijking met een soja-eiwitmaaltijd in het varken. Voeding 58: (5), 30 , 1997. 


\section{CURRICULUM VITAE}

Maaike Bruins werd op 20 april 1970 geboren in Chêne-Bougeries. In 1988 behaalde zij het WWO Atheneum-B diploma aan het Rijnlands Lyceum in Oegstgeest. Aansluitend studeerde zij Biologie aan de Rijksuniversiteit Leiden. In het kader van het Erasmus programma volgde zij haar eerste stage aan de Université de Toulouse bij het "Centre de Biologie du Développement" bij Prof. J.C. Smith. Bij de afdeling Dierfysiologie aan de Rijksuniversiteit Leiden van Prof. A. Addink verrichte zij onderzoek naar stikstofoxide productie in de karper. Vervolgens deed zij een onderzoeksstage naar de doorlaatbaarheid van endotheelcellagen bij de afdeling "vaat en bindweefsel onderzoek" van TNO bij Prof. V.W. van Hinsbergh. In 1994 werd de doctoraalstudie afgerond met het hoofdvak drug-targeting in tumors. Haar promotieonderzoek verrichte zij als $\mathrm{OlO}$ in het kader van een project dat gesubsidieerd werd door NWO (gebied medische wetenschappen), gedetacheerd aan de afdeling Algemene Heelkunde van de Universiteit van Maastricht. De resultaten van de studies onder leiding van Dr. N.E.P. Deutz, Prof. P.B. Soeters en Prof. W.H. Lamers zijn beschreven in dit proefschrift. Momenteel is ze werkzaam als postdoc bij de afdeling Biofarmacie (Prof. Dr. Th.J.C. van Berkel) binnen het Leiden/Amsterdam Center for Drug Research van de Rijksuniversiteit Leiden. 